\title{
DEFORMATION BEHAVIOR IN LIGHTWEIGHT ALLOYS: EFFECTS OF RARE-EARTH MICROALLOYING AND CARBON NANOTUBE REINFORCEMENT
}

\author{
by \\ FATMA MOKDAD \\ Master of Science in Aerospace and Mechanical Engineering \\ University of Arizona, Tucson, AZ, USA, 2013 \\ Bachelor of Science in Mechanical Engineering \\ A dissertation
presented to Ryerson University \\ in partial fulfillment of the \\ requirements for the degree of \\ Doctor of Philosophy \\ in the Program of \\ Mechanical and Industrial Engineering
}

Ecole de Technologie Superieure (ETS), Montreal, QC, Canada, 2011

Toronto, Ontario, Canada, 2017

(C) Fatma Mokdad 2017 


\section{AUTHOR'S DECLARATION}

I hereby declare that I am the sole author of this dissertation. This is a true copy of the dissertation, including any required final revisions, as accepted by my examiners.

I authorize Ryerson University to lend this dissertation to other institutions or individuals for the purpose of scholarly research.

I further authorize Ryerson University to reproduce this dissertation by photocopying or by other means, in total or in part, at the request of other institutions or individuals for the purpose of scholarly research.

I understand that my dissertation may be made electronically available to the public 


\title{
DEFORMATION BEHAVIOR IN LIGHTWEIGHT ALLOYS: EFFECT OF RARE-EARTH MICROALLOYING AND CARBON NANOTUBE REINFORCEMENT
}

\author{
(C) Fatma Mokdad, 2017 \\ Doctor of Philosophy \\ Department of Mechanical \& Industrial Engineering \\ Ryerson University
}

\begin{abstract}
One of the most important strategies for improving fuel efficiency and reducing anthropogenic emissions is vehicle lightweighting by the use of lightweight materials such as $\mathrm{Mg}$ and $\mathrm{Al}$ alloys in the automotive industry. The structural application of these alloys inevitably requires the mechanical properties and their continuous performance improvement to meet the increasingly stringent safety and durability requirements. An effective method to enhance the deformation resistance is to alloy with rare-earth (RE) elements for Mg alloys or develop composites with the addition of reinforcement for Al alloys. The objective of this dissertation was to identify the effects of RE element and carbon nanotube $(\mathrm{CNT})$ reinforcement on the deformation behavior, focusing mainly on the deformation mechanisms. The deformation behavior of a RE-free extruded AZ31 Mg alloy was first studied. It was observed that the propagation of distinct twin variants led to the confinement of the spaces constrained by the fine twin lamellas. Various double twinning structures acknowledged through atomistic simulations were experimentally observed via progressive electron backscatter diffraction (EBSD) analyses during stepwise
\end{abstract}


compression. The vanishing of primary $\{11 \overline{2} 1\}$ embryonic twins via the nucleation and growth of either single or multiple $\{10 \overline{1} 2\}$ secondary extension twins was detected, and two new ladder-like and branching-like twin-twin interaction phenomena were observed. Then a low-RE containing $\mathrm{Mg}$ alloy was exploited via texture and cyclic deformation studies. The addition of $0.2 \mathrm{wt} . \% \mathrm{Nd}$ in ZEK100-O Mg alloy led to a weaker basal texture in comparison with $\mathrm{AZ31} \mathrm{Mg}$ alloy. Fatigue life of ZEK100 alloy was longer than that of AZ31 alloy, due to a good combination of strength with ductility. Asymmetry of hysteresis loops was improved because of texture weakening and grain refinement, however anelastic behavior largely remained arising from the presence of twinning and detwinning. The last investigation involved deformation behavior of CNT reinforced $\mathrm{Al}$ composites where the addition of $2.0 \mathrm{wt} \% \mathrm{CNT}$ in a 2024Al alloy led to considerable grain refinement. Deformation resistance of the composite was effectively enhanced due to CNT load transfer, Hall-Petch strengthening, thermal mismatch and Orowan looping. In a nutshell, this work constitutes a valuable benchmark for understanding the factors affecting the performance of two lightweight alloys in the automotive and aerospace applications. 


\section{ACKNOWLEDGEMENTS}

I would like to express my sincere gratitude to my supervisor Dr. Daolun Chen for the great learning experience I gained during my $\mathrm{PhD}$, through his expertise and enthusiasm for the research topic. His continuous guidance, scientific rigorousness and encouragements would not only mark my $\mathrm{PhD}$ experience, but also my future professional life. I will always remember him telling us: "No mountain is too high, and no trouble is too difficult to overcome".

My deepest appreciation goes to my committee members for their honorable presence and their precious time in evaluating the proposed research. Their constructive criticism has helped me greatly in improving the quality of this dissertation.

I would like to thank the Natural Sciences and Engineering Research Council of Canada (NSERC), Premier's Research Excellence Award (PREA), NSERC-DAS Award, AUTO21 Network of Centers of Excellence, Automotive Partnership Canada (APC), Canada Foundation for Innovation (CFI), and Ryerson Research Chair (RRC) program for financial support. I would like to also thank Dr. Zongyi Ma (Shenyang National Laboratory for Materials Science) and his very helpful team including Dr. Z.Y. Liu, Dr. D.R. Ni, and Dr. B.L. Xiao, for supplying the Al base alloy and the Al/CNT reinforced composites. Many thanks to Professor A.A. Luo (Ohio State University) for the RE-free test material, Dr. T. Skszek and Dr. X.P. Niu (Magna International Inc.) and Professor M. Worswick (University of Waterloo) for the RE-Mg alloy. 
Sincere appreciation is owed to Messrs. Alan Machin, Joseph Amankrah, and Qiang Li for the great help in the specimen preparation and the use of equipment. I would like to also deeply thank them for their positive attitude, work ethics, amazing friendliness, and for being my family away from home. My great appreciation goes to Mr. Chao Ma for his unforgettable help in using the compression machine, Mr. Devin Ostrom for the easy access to the CNC Lab, Mr. Roy Churaman for the access to all facilities, and Mr. Andrew Heim for his help whenever needed.

I would like to thank all the faculty and staff members of the Mechanical and Industrial Engineering Department, as well as my current Lab colleagues: Andrew Macwan, Nabila Tahreen, Hongmei Ji, Yuntao $\mathrm{Li}$, and He Peng for contributing to the motivating work environment, and all my previous colleagues, mainly Dr. F.A. Mirza for his help and patience when I initially joined the group. I wish them all plenty of success in their future endeavors. 


\section{DEDICATION}

I dedicate this work to my parents Ridha and Sousou who have always been a great source of motivation, for their endless sacrifices and for teaching me that knowledge has no limits. To my siblings, Meriem for always being my role model, Med Tahar for constantly believing in me and Kerim for his unique spirit and care. To my nephews Dedo and Scan for their priceless presence. To my late grandparents: Baba Haj, Nana, Baba Cherif, and Bakhtouta for showing me the meaning of strength and perseverance, and to my uncle Tio Mokh for boosting my creativity since my early age. To my $2^{\text {nd }}$ family Hasna, Wahid, Chabouha, and Hamdoun for their support and encouragements. Last but certainly not least, to my husband Heikel for having always been there despite long distances, for giving me lasting loves, and for never doubting on my abilities. I would have not done it without him in my life. To the rest of my family and friends, for inspiring me. 
To

All cancer fighters

All over the world... 


\section{TABLE OF CONTENTS}

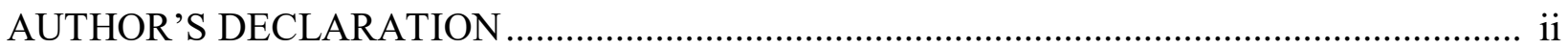

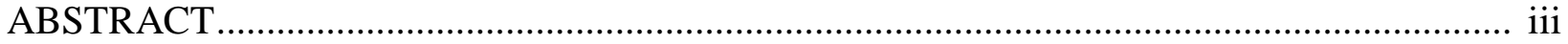

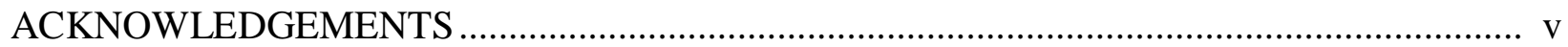

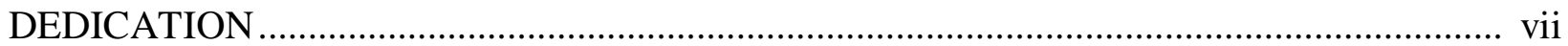

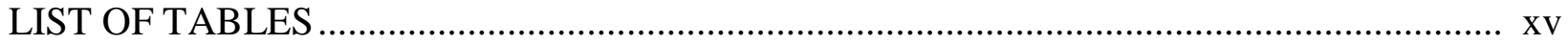

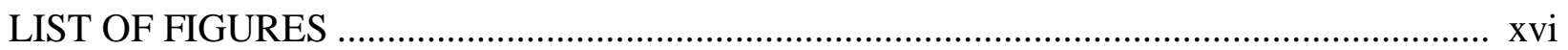

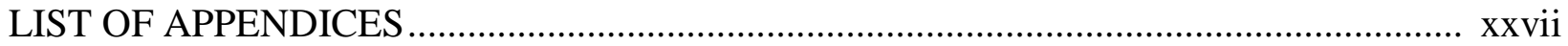

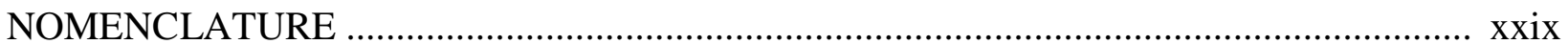

CHAPTER 1: INTRODUCTION .................................................................. 1

$1.1 \quad$ Background and motivation .......................................................................... 1

Objectives and scope of the dissertation .......................................................... 4

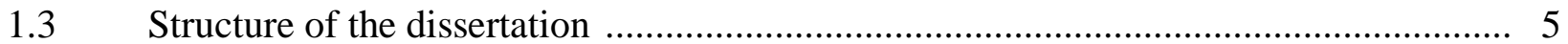

CHAPTER 2: LITERATURE REVIEW ............................................................. 7

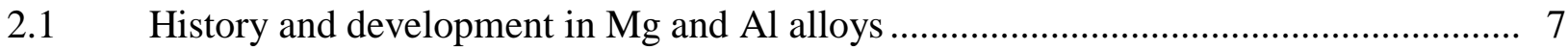

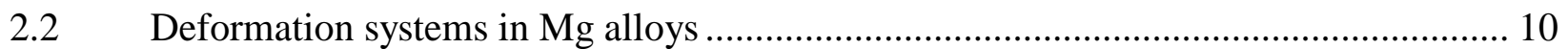

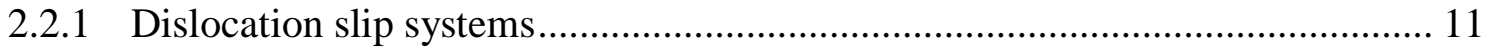

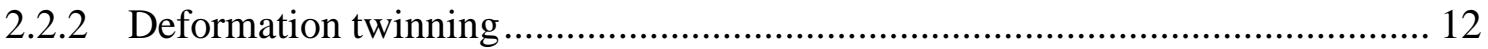

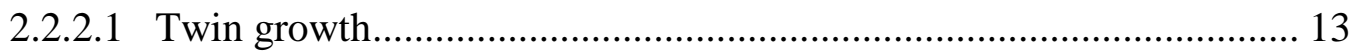

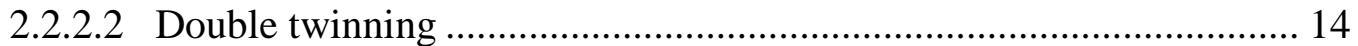

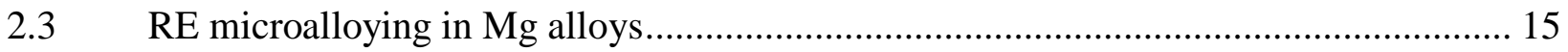

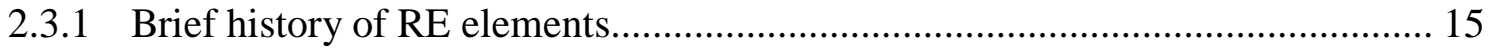

2.3.2 Major effects of $\mathrm{RE}$ elements on $\mathrm{Mg}$ alloys ................................................ 15 
2.3.2.1 Microstructure of RE-containing wrought Mg alloys ...................... 16

2.3.2.2 Crystallographic texture of RE-containing wrought $\mathrm{Mg}$ alloys .......... 17

2.3.3 Fatigue and anelastic behavior of RE-Mg alloys ..................................... 18

$2.4 \quad$ Low Nd-containing ZEK100 vs. RE-free AZ31 ............................................... 19

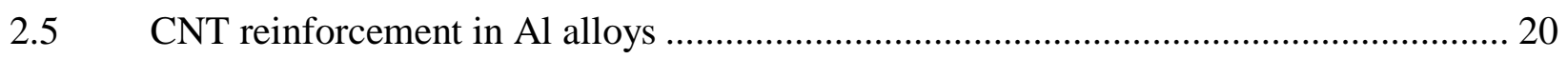

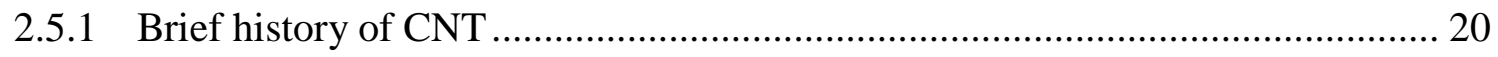

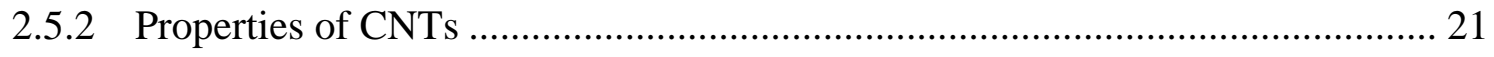

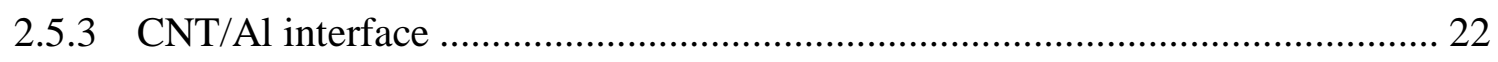

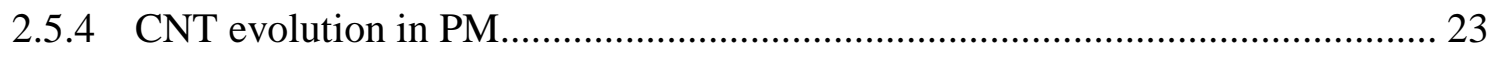

2.5.5 Functional performance of CNT/Al composite ...................................... 24

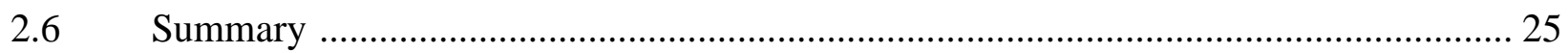

CHAPTER 3: EXPERIMENTAL PROCEDURE .................................................. 27

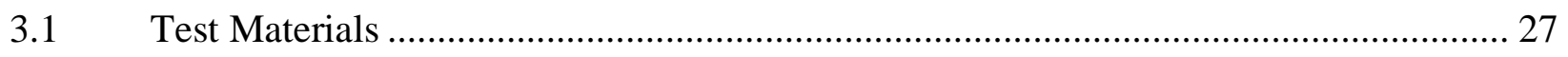

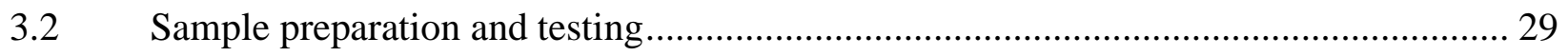

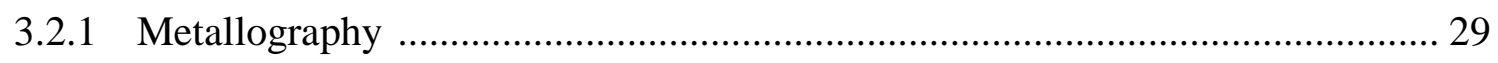

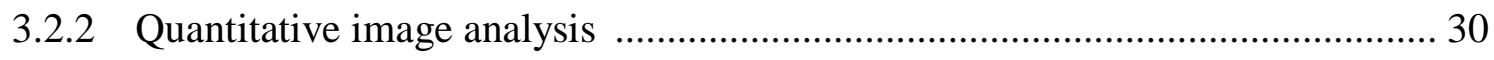

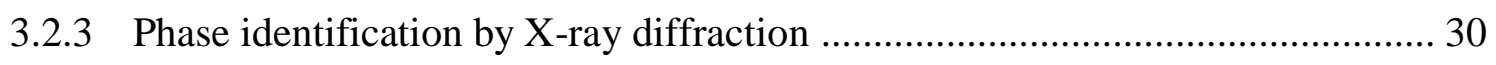

3.2.4 Texture measurements by X-ray diffraction ........................................ 31

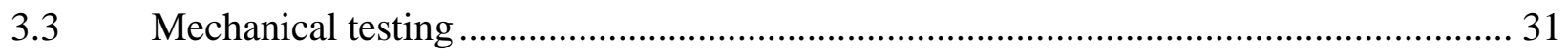

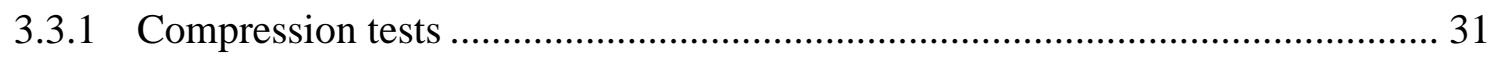

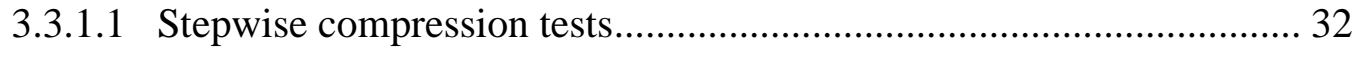

3.3.1.2 Continuous compression tests................................................ 33

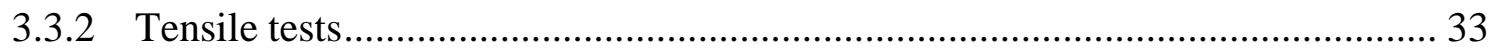


3.3.3 Fatigue tests

CHAPTER 4: DEFORMATION BEHAVIOR OF A RE-FREE AZ31 Mg ALLOY

DURING STEPWISE COMPRESSION ........................................... 37

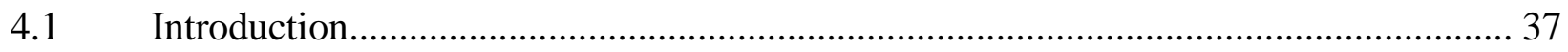

4.2 Microstructural characterization upon compression ............................................. 37

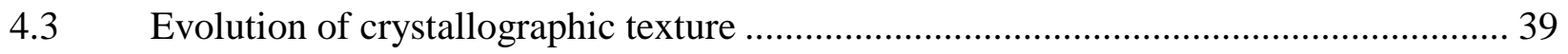

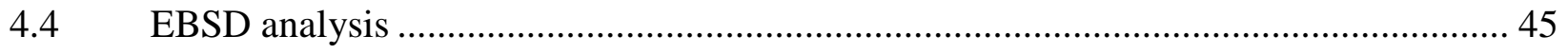

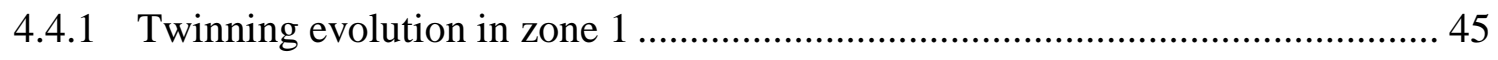

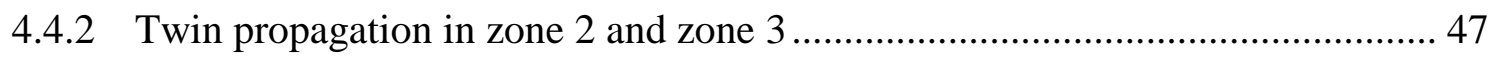

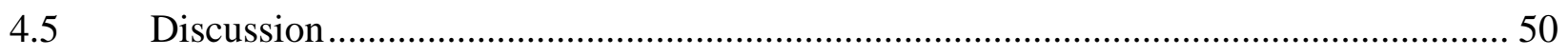

4.5.1 Effect of the grain shape on the growth of twins ...................................... 50

4.5.2 Growth of $\left\{10 \overline{1}_{2}\right\}$ extension twins under similar and distinct twin variants ....... 53

4.5.3 Distinctive characteristics of $\{10 \overline{1} 2\}$ twin-twin interactions.............................58

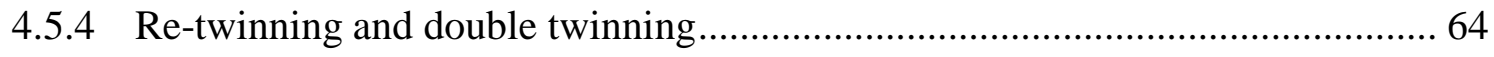

4.5.5 Feasibility of crossing between different types of twins .............................. 71

4.5.6 Theoretical analysis of twin-twin interactions ....................................... 73

4.5.7 Contribution of twinning to the deformation behavior ................................ 75

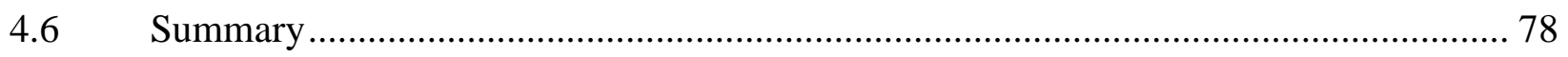

CHAPTER 5: CYCLIC DEFORMATION OF A LOW RE-CONTAINING ZEK100 Mg

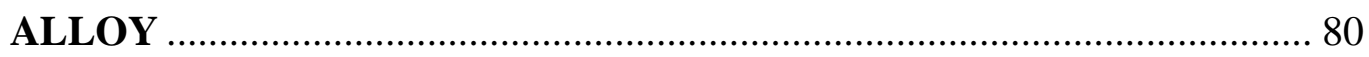

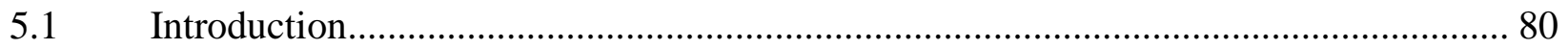

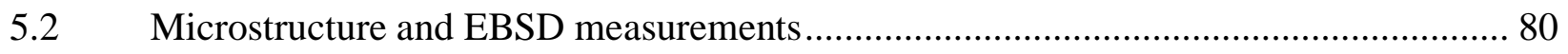

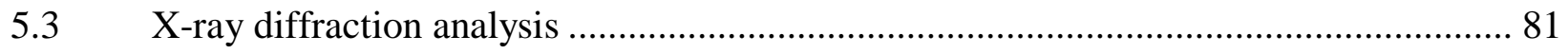




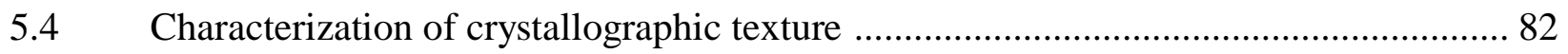

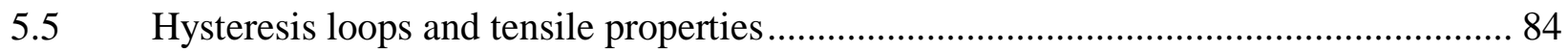

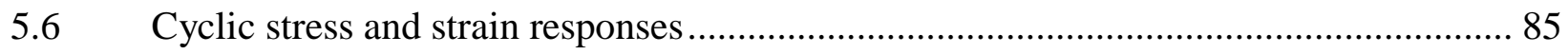

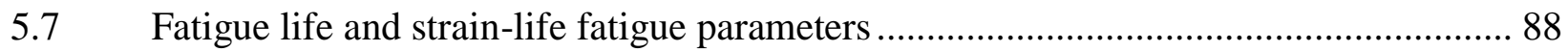

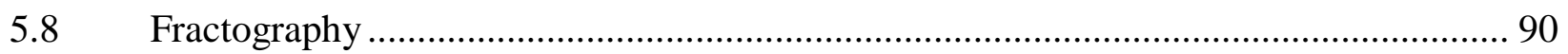

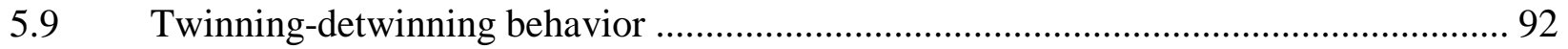

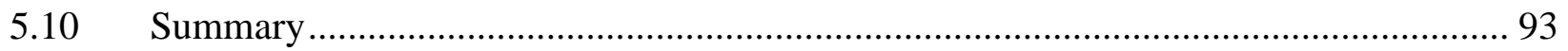

CHAPTER 6: ANELASTIC BEHAVIOR AND EFFECT OF STRAIN RATIO ON CYCLIC DEFORMATION OF ZEK100 Mg ALLOY ............................ 95

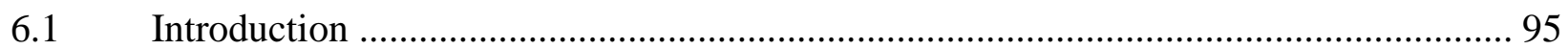

6.2 Evolution of fatigue behavior with strain ratio .................................................... 95

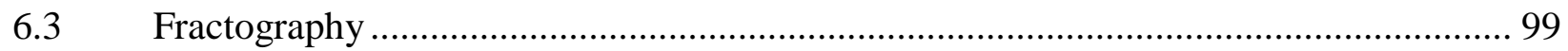

6.4 Quantitative analysis of the anelastic behavior ................................................. 99

6.4.1 Effect of strain ratio on the anelastic behavior ........................................... 101

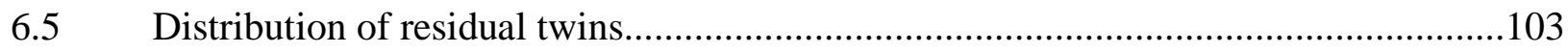

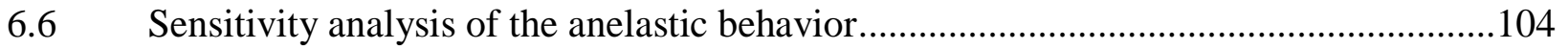

6.6.1 Theoretical foundation ........................................................................ 104

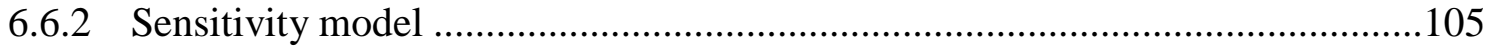

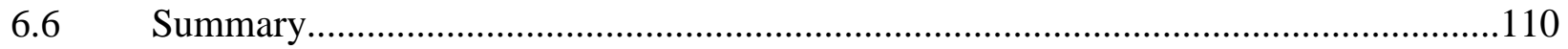

CHAPTER 7: DEFORMATION AND STRENGTHENING MECHANISMS OF THE 2024AI ALLOY AND THE 2.0 WT.\% CNT 2024Al COMPOSITE.......... 111

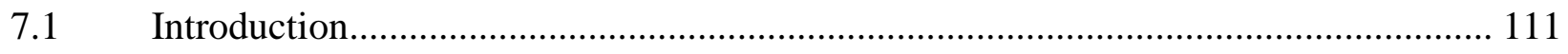

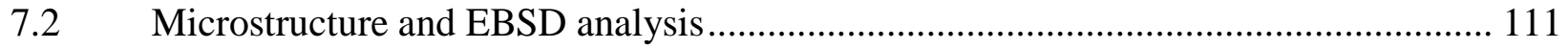




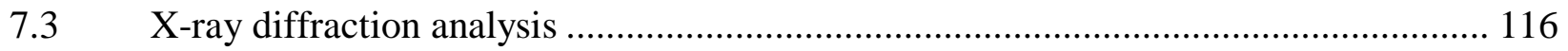

7.3.1 Lattice parameter of the alloy and composite ...................................................117

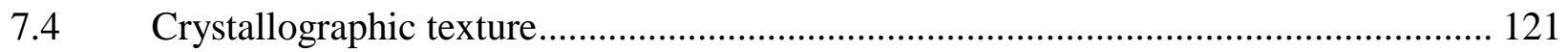

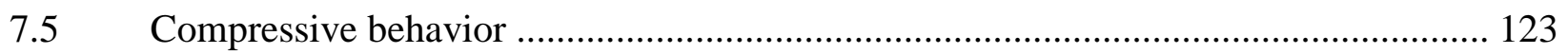

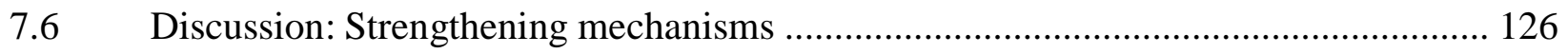

7.6.1 Grain refinement and grain boundary strengthening .......................................127

7.6.2 Recrystallization and built-up of substructures...............................................129

7.6.3 Zener drag effect ...............................................................................130

7.6.4 Load transfer, thermal mismatch and Orowan looping ……….........................130

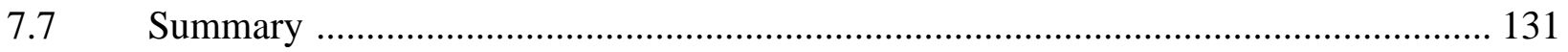

CHAPTER 8: HOT DEFORMATION BEHAVIOR OF 2024AI BASE ALLOY AND 2.0 WT.\% CNT REINFORCED 2024 Al COMPOSITE ................................. 133

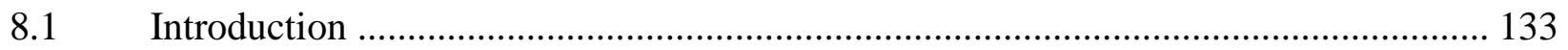

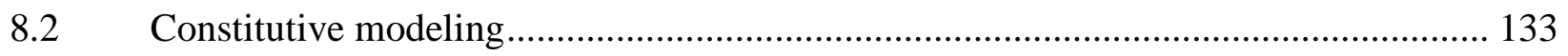

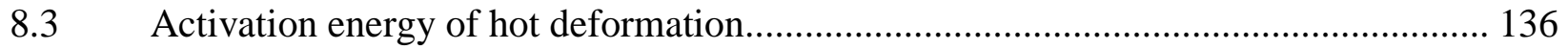

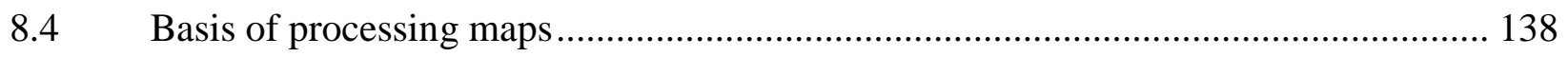

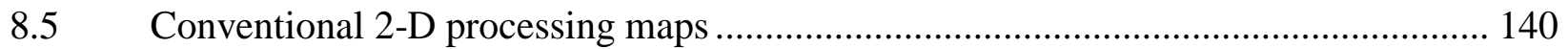

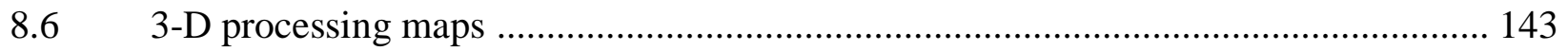

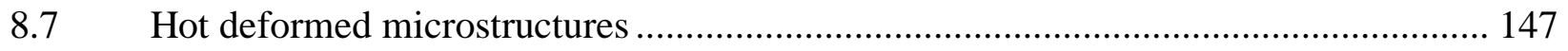

8.7.1 Deformed microstructures in the "unstable" domain...........................................147

8.7.1.1 Deformed 2024Al samples ..........................................................148

8.7.1.2 Deformed 2.0wt.\% CNT/2024Al samples..........................................150 
8.7.2 Deformed microstructures in the "stable" domain.

8.7.2.1 Deformed 2024Al samples

8.7.2.2 Deformed 2.0wt.\% CNT/2024Al samples.....

8.8 Summary 160

CHAPTER 9: SUMMARY, CONCLUSIONS AND FUTURE WORK 162

9.1 Conclusions. 162

9.2 Main contributions 164

9.3 Recommendations for future work 166 APPENDICES: HOT DEFORMATION FLOW CURVES AT ALL STRAIN RATES AND TEMPERATURES FOR THE ALLOY AND THE COMPOSITE 168 REFERENCES 173 


\section{LIST OF TABLES}

Table 2.1 Physical properties of the materials used in this study compared to others ......... 9

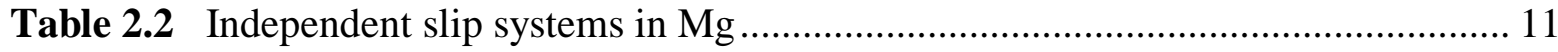

Table 2.3 Properties of carbon materials................................................................... 21

Table 3.1 Chemical composition of AZ31Mg alloy (wt.\%). ...................................... 27

Table 3.2 Chemical composition of ZEK100 Mg alloy (wt.\%). ................................. 28

Table 3.3 Chemical composition of 2024Al alloy (wt.\%). ..................................... 28

Table 3.4 Test parameters under different strain ratios at strain amplitude of $0.8 \%$ and a

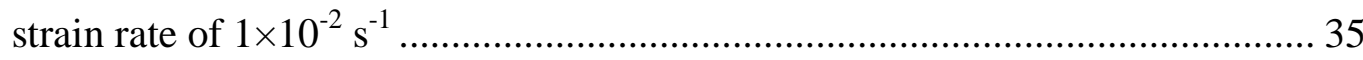

Table 4.1 Main texture components identified from Euler angles in accordance with the specified crystal coordinate system in Fig.4.3(b) . ................................... 44

Table 5.1 Low cycle fatigue parameters obtained for the rolled ZEK100 Mg alloy ......... 90

Table 6.1 Six-dimensional input factor set for sensitivity analysis of the anelastic behavior.

Table 7.1 Cohen method parameters for the determination of lattice constants ao of the 2024Al alloy and 2.0 wt.\% CNT/2024Al composite, respectively.

Table 8.1 Main parameters of the hot deformed 2024Al alloy and the 2.0 wt.\% CNT/2024Al composite evaluated from the constitutive equations. 


\section{LIST OF FIGURES}

Fig.2.1 (a) Hexagonal close packed (hcp) crystal structure, and (b) slip directions.... 10

Fig.2.2 (a) (a) Basal- $\langle a\rangle$, (b) prismatic- $\langle a\rangle$, (c) pyramidal- $\langle a\rangle$, and (d) pyramidal$<c+a>$ slip systems in $\mathrm{Mg}$ 11

Fig.2.3 (a) Schematic diagram showing how twinning results from an applied shear stress $\tau$, and (b) open circles represent atoms that did not change position; dashed and solid circles represent original and final atom positions, respectively

Fig.2.4 Optical images of the as-extruded specimens of (a) Mg-8Gd-0.4Zr alloy, (b) Mg-8Gd-1Zn-0.4Zr alloy, and (c) Mg-8Gd-3Zn-0.4Zr alloy ......................... 16

Fig.2.5 Effect of Gd content on the texture intensities of an Mg-Gd alloy .................. 18

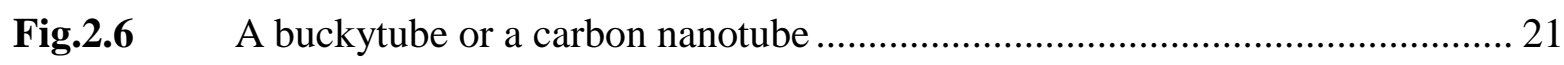

Fig.2.7 SEM image of a nanotube bundle to be pulled for tensile strength experiment22

Fig.2.8 (a) Applied total force versus the displacement of the rope, (b) force applied to the nanotube rope versus its displacement, (c) conductance (the inverse of the resistance) of the nanotube rope versus its displacement, and (d) uniaxial stress applied to the nanotube rope versus the strain (elongation) 23

Fig.3.1 Illustration of the specimen for compression testing on AZ31 Mg alloy (unit in $\mathrm{mm})$. 32

Fig.3.2 Illustration of the specimen for compression testing on $2024 \mathrm{Al}$ and the $2.0 \mathrm{wt} . \%$ CNT/2024 Al composite 33

Fig.3.3 Illustration of the dimensions of the sub-sized tensile test specimens 34

Fig.3.4 Illustration of the dimensions of the sub-sized fatigue test specimens. 35 
Fig.4.1 Typical compressive true stress-true strain curve of the extruded AZ31 Mg alloy along with microstructural changes at varying compressive true strains of (a) $0 \%$, (b) $2.5 \%$, (c) $4.3 \%$, (d) $6.0 \%$, (e) $8.4 \%$, and (f) $10.8 \%$ 38

Fig.4.2 \{0001\}pole figures obtained from (a) the undeformed sample and the compressed samples of the extruded AZ31 Mg alloy at true strain amounts of (b) $2.5 \%$, (c) $4.3 \%$, (d) $6.0 \%$, (e) $8.4 \%$ and (f) $10.8 \%$ 40

Fig.4.3 (a) ODF sections at $\varphi_{2}=0^{\circ}$ and $\varphi_{2}=30^{\circ}$ of samples compressed at $0 \%, 2.5 \%$, $4.3 \%, 6.0 \%, 8.4 \%$ and $10.8 \%$, (b) definition of the crystal coordinate system based on the rotation of the specimen coordinate system through the Euler angles $\varphi_{1}, \Phi, \varphi_{2}$ in accordance with the Bunge definition in the order of 1,2,3, and (c) evolution of main texture components with the strain.

Fig.4.4 Normal-projected EBSD orientation maps and the corresponding inverse pole figures of zone 1 strained along ED at (a) $0 \%$, (b) $2.5 \%$, (c) $4.9 \%$, (d) $6.8 \%$ and (e) $8.6 \%$. 46

Fig.4.5 EBSD forward scatter detector (FSD) images and corresponding normalprojected orientation maps of zone 2 strained at (a, b) $0 \%$, (c, d) $4.9 \%$ and (e, f) $6.8 \%$, respectively. 48

Fig.4.6 Normal-projected EBSD orientation maps of zone 3 strained along ED at (a) $0 \%$, (b) $1.2 \%$, (c) $2.5 \%$, (d) $5.5 \%$ and (e) $8 \%$ 49

Fig.4.7 Twinning sequence of zone 1- grain 1 by means of characteristic misorientation profiles between points $\mathrm{A}$ and $\mathrm{B}$ specified on the orientation maps, at true strains of (a, b) $0 \%,(c, d) 2.5 \%$, and (e, f) $4.9 \%$, respectively. 51 
Fig.4.8 $\{10 \overline{1} 2\}$ twin-twin interactions in zone 1 - grain 2: (a) orientation map of the initial twin-free microstructure, and twin growth during stepwise compression of (b) $2.5 \%$ and (c) $4.9 \%$; the lattice orientation is represented by schematic hcp unit cells, and (d) schematic illustration. 52

Fig.4.9 (a) Optical micrograph of a grain which experienced one variant of $\{10 \overline{1} 2\}$ extension twinning with (b) the hcp unit cell plotted to detect the active twin planes and their traces. EBSD orientation maps of zone 2 - grain 1 showing the growth of $\left\{10 \overline{1}_{2}\right\}$ twin variant at true strains of (c) $0 \%$, (d) $4.9 \%$ and (e) $6.8 \%$, respectively, and (f) the corresponding $\{0001\}$ pole figure of the matrix and twins. 54

Fig.4.10 (a) Optical micrograph of a grain which experienced two variants of $\{10 \overline{1} 2\}$ extension twinning with (b) the hcp unit cell plotted to detect the active twin planes and their traces. EBSD orientation maps of zone 2 - grain 2 showing the growth of two $\{10 \overline{1} 2\}$ twin variants at true strains of (c) $0 \%$, (d) $4.9 \%$ and (e) $6.8 \%$, respectively, and (f) the corresponding $\{0001\}$ pole figure of the matrix and twins. 55

Fig.4.11 (a) EBSD Euler angle map of zone 2 - grain 2 (Fig.11(e)) strained at $6.8 \%$, (b) orientation relationships between matrix, twins and neighboring grains presented in the 3D Euler space based on the provided Euler color code, and (c) Euler angles of the matrix and the twins propagating under two different variants. 57

Fig.4.12 (a) Optical micrograph illustrating type A twin-twin interaction (ladder-like structure), and (b) schematic diagram. EBSD orientation maps of zone 3 - grain 
1 showing the growth of the ladder-like structure at true strains of (c) $1.2 \%$, (d) $2.5 \%$, (e) $5.5 \%$ and (f) $8 \%$, respectively, and (g) the corresponding $\{0001\}$ pole figure of the matrix and the twins. 60

Fig.4.13 (a) Optical micrograph illustrating type B twin-twin interaction (branching-like structure), (b) the corresponding hcp unit cell plotted with respect to the stress axis, and active twin planes and their traces in (c) $V_{1}$, (d) $V_{2}$, and (e) $V_{3}$, and (f) Schmid factors for different variants of extension twinning

Fig.4.14 EBSD orientation maps showing the growth of the branching-like structure in zone 3 - grain 2 at true strains of (a) $0 \%$, (b) $1.2 \%$, (c) $2.5 \%$, (d) $5.5 \%$ and (e) $8 \%$, and (f) the corresponding $\{0001\}$ pole figure of the matrix and the twins.63

Fig.4.15 (a) Optical micrograph of a grain which experienced $\left\{10 \overline{1}_{2}\right\}$ double twinning with the same twin variant as the parent twin, and (b) the corresponding schematic illustration. EBSD orientation maps of zone 2 - grain 3 at true strains of (c) $0 \%$, (d) $4.9 \%$ and (e) $6.8 \%$, respectively, (d) the corresponding $\{0001\}$ pole figure of the matrix and twins, and misorientation profiles across the boundaries of $(\mathrm{g})$ the parent twin $\mathrm{T}_{1}$, and $(\mathrm{h})$ the secondary twin $\mathrm{T}_{2} \ldots \ldots \ldots \ldots \ldots 66$

Fig.4.16 (a) Optical micrograph of a grain which experienced $\{10 \overline{1} 3\}-\{10 \overline{1} 2\}$ double twin and (b) the corresponding schematic illustration. EBSD orientation maps of zone $3-\mathrm{P}_{1}$ (Fig.7(e)) compressed at (c) 5.5\%, and (d) 8\%, showing the formation of the $\{10 \overline{1} 3\}-\{10 \overline{1} 2\}$ double twin, which is confirmed through (e) the corresponding $\{0001\}$ pole figure of the matrix and the twins, and the misorientation profiles across the boundaries (f) $b_{1}$ and (h) $b_{2}$ of the parent twin $\mathrm{T}_{1}$ and $(\mathrm{g})$ the secondary twin $\mathrm{T}_{2}$. 68 
Fig.4.17 (a) Initial EBSD orientation map of zone 3 - grain 3, along with the deformed maps (observational plane and color legend displayed in Fig.4) and their corresponding $\{0001\}$ pole figures of the matrix and the twins at true strain

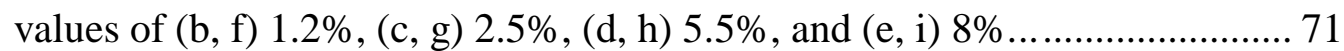

Fig.4.18 EBSD orientation maps of zone $3-\mathrm{P}_{2}$ compressed at (a) $5.5 \%$ and (b) $8 \%$, showing the formation of a crossing-like twin structure between a thin $\left\{\begin{array}{ll}10 & 1\end{array}\right\}$ contraction twin which encounters two merged $\{10 \overline{1} 2\}$ extension twins. Schematic illustrations of (c) the case of an actual crossing and (d) the case of an apparent crossing between twins are also provided. 72

Fig.4.19 Formation process of twin-twin boundaries: (a) two twins approaching the boundary of a pre-existing twin, (b) impingement of twinning dislocations on the twin boundary, (c) zipping process where dislocations between the twins in contact form common junctions, and (d) dissociation where twinning dislocations associated with one twin dissociate into twinning dislocations of the other twin. 74

Fig.5.1 (a) Geometry and dimensions (in $\mathrm{mm}$ ) of fatigue test specimens, and (b) threedimensional EBSD band contrast (BC) mapping of the rolled ZEK100 Mg alloy in an annealed state, where RD stands for the rolling direction, TD denotes the transverse direction, and ND indicates the normal direction 81

Fig.5.2 EBSD orientation map on the RD-TD plane of the rolled ZEK100 Mg alloy . 82

Fig.5.3 X-ray diffraction pattern of the ZEK100 Mg alloy ................................... 83

Fig.5.4 SEM back-scattered electron image specifying EDS point analysis locations and the corresponding composition at particles $\mathrm{A}$ and $\mathrm{B}$, respectively..... 83 
Fig.5.5 (0001), (10) 10$)$ and $(10 \overline{1} 1)$ pole figures of the rolled ZEK100 Mg alloy, where RD stands for the rolling direction, TD for the transverse direction, and ND for the normal direction 84

Fig.5.6 Characteristic stress-strain hysteresis loops of the (a) first cycle, (b) second cycle, and (c) mid-life cycle at a total strain amplitude of $1.2 \%$ and strain ratio $R_{\mathrm{S}}=-1$ for the rolled ZEK100 alloy, in comparison with the extruded AM30 and GW103K alloys 86

Fig.5.7 Stress amplitude vs. the number of cycles at different total strain amplitudes. 87

Fig.5.8 Plastic strain amplitude vs. the number of cycles at different total strain amplitudes 87

Fig.5.9 Total strain amplitude as a function of the number of cycles to failure for the rolled ZEK100 Mg alloy, in comparison with the data reported in the literature for various wrought $\mathrm{Mg}$ alloys 88

Fig.5.10 Evaluation of strain-life fatigue parameters in the form of log-elastic, plastic and total strain amplitudes vs. log-number of reversals to failure, respectively 90

Fig.5.11 SEM micrographs of overall fracture surfaces of the specimens fatigued at a strain amplitude of (a) $0.4 \%$ and (b) $1.0 \%$, respectively 91

Fig.5.12 SEM micrographs of the fatigue crack propagation area in the specimens fatigued at strain amplitude of (a) $0.4 \%$ and (b) $1.0 \%$, respectively 91

Fig.5.13 (a) Optical micrographs of the areas near the fracture surface, showing the overall distribution of residual twins in a sample of ZEK100 alloy fatigued at a 
strain amplitude of $1.2 \%$ with emphasis on two regions (b) and (c), respectively 93

Fig.6.1 Stress-strain hysteresis loops of the rolled ZEK100 Mg alloy tested at different strain ratios at a total strain amplitude of $0.8 \%$, (a) first cycle, (b) second cycle, and (c) mid-life cycle 96

Fig.6.2 (a) Stress amplitude and (b) plastic strain amplitude vs. the number of cycles at different strain ratios, at a total strain amplitude of $0.8 \%$ 97

Fig.6.3 Number of cycles to failure vs. strain ratio for the rolled ZEK100 alloy tested at a total strain amplitude of $0.8 \%$ and a strain rate of $1 \times 10^{-2} \mathrm{~s}^{-1}$ 98

Fig.6.4 Typical SEM images of the overall fracture surfaces of the specimens fatigued at total strain amplitude of $0.8 \%$ and at a strain ratio of (a) 0.5 and (b) $-\infty \ldots . .99$

Fig.6.5 Schematic illustration showing the parameters defined to characterize the anelastic behavior in tension and compression by means of a stress-strain hysteresis loop 100

Fig.6.6 (a) Eccentricity along the strain axis $e$, (b) relative slope change $d$, and (c) angle deviation $\Delta \alpha$ as a function of strain ratio for ZEK100 tested at a strain rate of $1 \times 10^{-2} \mathrm{~s}^{-1}$ at a total strain amplitudes of $0.8 \%$ for the second and mid-life cycles, respectively 102

Fig.6.7 Micrographs of the areas near the fracture surface fatigued at (a) $R_{\varepsilon}=0.5$, and (b) $R_{\varepsilon}=-\infty$ 103

Fig.6.8 Representation of the global sensitivity analysis performed to identify the most influencing factors on the anelastic behavior 105 
Fig.6.9 (a) Mid-life total strain amplitude, and (b) relative error as a function of strain ratio in the fatigue tests of ZEK100 alloy at a strain rate of $1 \times 10^{-2} \mathrm{~s}^{-1}$ 107

Fig.6.10 Influencing inputs on the anelastic behavior by means of (a) Pearson Linear Correlation Coefficients (PLCC), and (b) Spearman Rank order Correlation Coefficients (SRCC) 109

Fig.7.1 Typical SEM micrographs showing the details of microstructural features of the 2024Al alloy and 2.0 wt.\% CNT/2024Al composite at (a, c) lower and (b, d) higher magnifications, respectively. Yellow coarse arrows indicate $\mathrm{Cu}-$ containing particles, and red thin arrows designate nanoparticles 112

Fig.7.2 (a) A typical SEM back-scattered electron image, and (b) EDS line scan results across a position indicated by the line in (a) for the $2.0 \mathrm{wt} . \% \mathrm{CNT} / 2024 \mathrm{Al}$ composite

Fig.7.3 (a) A typical TEM image of the 2.0 wt.\% CNT/2024Al composite. Red thin arrows indicate singly dispersed CNTs, and blue thick arrows designate the $\mathrm{Al}_{4} \mathrm{C}_{3}$ phase, (b) HRTEM image showing the interface structure between the $\mathrm{Al}$ matrix and CNTs 114

Fig.7.4 EBSD orientation maps of (a) the 2024Al alloy, and (b) the 2.0 wt.\% CNT/2024Al composite. 115

Fig.7.5 X-ray diffraction patterns of the 2024Al alloy and 2.0 wt.\% CNT/2024Al composite. 116

Fig.7.6 $\{100\},\{110\}$, and $\{111\}$ pole figures of the 2024Al alloy based on (a, f, k) XRD results and (b, g, l) EBSD results along with the 2.0 wt.\% CNT/2024Al composite based on (c, h, m) XRD results and (d, i, n) EBSD results, 
respectively, where ED stands for the extrusion direction, and RD denotes the radial direction, and $(\mathrm{e}, \mathrm{j}, \mathrm{o})$ indicate schematically the texture components. 121

Fig.7.7 Compressive true stress-true strain curves of the 2024Al alloy (continuous lines) and the $2.0 \mathrm{wt} \% \mathrm{CNT} / 2024 \mathrm{Al}$ composite (dashed lines): (a) at a strain rate of $0.001 \mathrm{~s}^{-1}$ and varying temperatures, and (b) at $300^{\circ} \mathrm{C}$ and varying strain rates 124

Fig.7.8 Change of compressive yield strength (CYS) with deformation temperature and strain rate of (a) the 2024Al alloy, and (b) the $2.0 \mathrm{wt} \% \mathrm{CNT} / 2024 \mathrm{Al}$ composite. Strain rates are expressed in $\mathrm{s}^{-1}$ 126

Fig.7.9 (a) Grain boundary mapping along with (b) the misorientation angle histogram for the $2.0 \mathrm{wt} . \% \mathrm{CNT} / 2024 \mathrm{Al}$ composite 128

Fig.8.1 The relationship of CYS, strain rate and temperature of the base 2024Al alloy (continuous lines) and the 2.0 wt.\% CNT/2024Al (dashed lines): (a) $\ln (\dot{\varepsilon})$ versus $\ln (\sigma)$, (b) $\ln (\dot{\varepsilon})$ versus $\sigma$, and (c) $\ln (\dot{\varepsilon})$ versus $\ln [\sinh (\alpha \sigma)]$ 135

Fig.8.2 Relationship of CYS, strain rate and temperature in terms of $\ln [\sinh (\alpha \sigma)]$ versus $1000 / T$ (a) for the 2024Al alloy, and (b) for the 2.0 wt.\% CNT/2024Al composite. Strain rates are expressed in $\mathrm{s}^{-1}$ 138

Fig.8.3 Processing maps of the 2024Al alloy for the true strain values of (a) $\varepsilon=0.1$, (b) $\varepsilon=0.3$, and $(\mathrm{c}) \varepsilon=0.5$, respectively 141

Fig.8.4 Processing maps of the $2.0 \mathrm{wt} . \% \mathrm{CNT} / 2024 \mathrm{Al}$ composite for the true strain values of (a) $\varepsilon=0.1$, (b) $\varepsilon=0.3$, and (c) $\varepsilon=0.5$, respectively 142

Fig.8.5 3-D power dissipation maps of (a) the 2024Al alloy, and (b) the 2.0 wt.\% $\mathrm{CNT} / 2024 \mathrm{Al}$ composite sliced at true strain values of $\varepsilon=0.1, \varepsilon=0.3$, and $\varepsilon=$ 0.5 , respectively 144 
Fig.8.6 3-D power dissipation maps of (a) the 2024Al alloy, and (b) the $2.0 \mathrm{wt} . \%$ CNT/2024Al composite sliced at temperature values of 200,300 , and $400^{\circ} \mathrm{C}$, respectively 145

Fig.8.7 3-D power dissipation maps of (a) the 2024Al alloy, and (b) the 2.0 wt.\% CNT/2024Al composite sliced at strain rate values of $0.001 \mathrm{~s}^{-1}(\log (\dot{\varepsilon})=-3)$, $0.01 \mathrm{~s}^{-1}(\log (\dot{\varepsilon})=-2)$, and $0.1 \mathrm{~s}^{-1}(\log (\dot{\varepsilon})=-1)$, respectively 146

Fig.8.8 3-D flow instability maps of (a) the 2024Al alloy, and (b) the 2.0 wt.\% $\mathrm{CNT} / 2024 \mathrm{Al}$ composite sliced at strain rate values of $0.01 \mathrm{~s}^{-1}(\log (\dot{\varepsilon})=-2)$, $0.03 \mathrm{~s}^{-1}(\log (\dot{\varepsilon})=-1.5)$, and $0.1 \mathrm{~s}^{-1}(\log (\dot{\varepsilon})=-1)$, respectively 147

Fig.8.9 Typical SEM micrograph showing (a) the overall microstructural features of the 2024Al alloy deformed at a strain rate of $0.1 \mathrm{~s}^{-1}$ and $200^{\circ} \mathrm{C}$. Magnified micrographs of (b) zone1 and (c) zone2 are also provided 148

Fig.8.10 Second-phase particle cracking in the 2024Al base alloy compressed at $0.1 \mathrm{~s}^{-1}$ and $200^{\circ} \mathrm{C}$ : (a) SEM micrograph, and (b) schematic representation 150

Fig.8.11 Typical SEM micrograph showing (a) microstructural features of the $2.0 \mathrm{wt} . \%$ $\mathrm{CNT} / 2024 \mathrm{Al}$ composite deformed at a strain rate of $0.1 \mathrm{~s}^{-1}$ and $200^{\circ} \mathrm{C}$. Magnified view provided in (b) 151

Fig.8.12 Typical SEM micrograph showing (a) second-phase particle rotation of the 2.0 wt.\% CNT/2024Al composite deformed at a strain rate of $0.1 \mathrm{~s}^{-1}$ and $400^{\circ} \mathrm{C}$, (b) schematic representation, and (c) magnified view 152

Fig.8.13 EBSD orientation maps along with grain diameter distribution histograms of the 2024Al alloy deformed at a strain rate of $0.001 \mathrm{~s}^{-1}$ and (a, b) $300^{\circ} \mathrm{C}$, (c, d) $350^{\circ} \mathrm{C}$, and $(\mathrm{e}, \mathrm{f}) 400^{\circ} \mathrm{C}$ 155 
Fig.8.14 EBSD recrystallized fraction component maps along with their quantification histograms of the 2024Al alloy deformed at a strain rate of $0.001 \mathrm{~s}^{-1}$ and $(\mathrm{a}, \mathrm{b})$

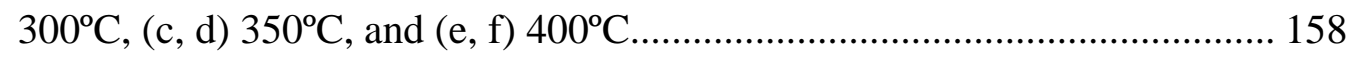

Fig.8.15 $\{110\}$ pole figures of the $2.0 \mathrm{wt} \% \mathrm{CNT} / 2024 \mathrm{Al}$ composite at (a) the undeformed state, and compressed at a strain rate of $0.001 \mathrm{~s}^{-1}$ and (b) $300^{\circ} \mathrm{C}$, (c) $350^{\circ} \mathrm{C}$, and $(\mathrm{d}) 400^{\circ} \mathrm{C}$. 159 


\section{LIST OF APPENDICES}

A 1.1 Compressive flow curves of two distinct reproducible tests for the 2024Al alloy compressed at RT and strain rates of (a) $10^{-3} \mathrm{~s}-1$, (b) $10^{-2} \mathrm{~s}^{-1}$, and (c) $10^{-1} \mathrm{~s}^{-1}$. . .168

A 1.2 Compressive flow curves of two distinct reproducible tests for the 2024Al alloy compressed at $200^{\circ} \mathrm{C}$ and strain rates of (a) $10^{-3} \mathrm{~s}-1$, (b) $10^{-2} \mathrm{~s}^{-1}$, and (c) $10^{-1} \mathrm{~s}^{-1}$. 168

A 1.3 Compressive flow curves of two distinct reproducible tests for the 2024Al alloy compressed at $250^{\circ} \mathrm{C}$ and strain rates of (a) $10^{-3} \mathrm{~s}-1$, (b) $10^{-2} \mathrm{~s}^{-1}$, and (c) $10^{-1} \mathrm{~s}^{-1}$.

A 1.4 Compressive flow curves of two distinct reproducible tests for the 2024Al alloy compressed at $300^{\circ} \mathrm{C}$ and strain rates of (a) $10^{-3} \mathrm{~s}-1$, (b) $10^{-2} \mathrm{~s}^{-1}$, and (c) $10^{-1} \mathrm{~s}^{-1}$.

A 1.5 Compressive flow curves of two distinct reproducible tests for the 2024Al alloy compressed at $350^{\circ} \mathrm{C}$ and strain rates of (a) $10^{-3} \mathrm{~s}-1$, (b) $10^{-2} \mathrm{~s}^{-1}$, and (c) $10^{-1} \mathrm{~s}^{-1}$.

A 1.6 Compressive flow curves of two distinct reproducible tests for the 2024Al alloy compressed at $400^{\circ} \mathrm{C}$ and strain rates of (a) $10^{-3} \mathrm{~s}-1$, (b) $10^{-2} \mathrm{~s}^{-1}$, and (c) $10^{-1} \mathrm{~s}^{-1}$. 170

A 1.7 Compressive flow curves of two distinct reproducible tests for the 2.0 wt.\% CNT 2024Al composite compressed at RT and strain rates of (a) $10^{-3} \mathrm{~s}-1$, (b) $10^{-}$ $2 \mathrm{~s}^{-1}$, and (c) $10^{-1} \mathrm{~s}^{-1}$ 170 
A 1.8 Compressive flow curves of two distinct reproducible tests for the $2.0 \mathrm{wt} . \%$ CNT 2024Al composite compressed at $200^{\circ} \mathrm{C}$ and strain rates of (a) $10^{-3} \mathrm{~s}-1$, (b) $10^{-2} \mathrm{~s}^{-1}$, and (c) $10^{-1} \mathrm{~s}^{-1}$ 170

A 1.9 Compressive flow curves of two distinct reproducible tests for the $2.0 \mathrm{wt} . \%$ CNT 2024Al composite compressed at $250^{\circ} \mathrm{C}$ and strain rates of (a) $10^{-3} \mathrm{~s}-1$, (b) $10^{-2} \mathrm{~s}^{-1}$, and (c) $10^{-1} \mathrm{~s}^{-1}$

A 1.10 Compressive flow curves of two distinct reproducible tests for the $2.0 \mathrm{wt} . \%$ CNT 2024Al composite compressed at $300^{\circ} \mathrm{C}$ and strain rates of (a) $10^{-3} \mathrm{~s}-1$, (b) $10^{-2} \mathrm{~s}^{-1}$, and (c) $10^{-1} \mathrm{~s}^{-1}$ 171

A 1.11 Compressive flow curves of two distinct reproducible tests for the $2.0 \mathrm{wt} . \%$ CNT 2024Al composite compressed at $350^{\circ} \mathrm{C}$ and strain rates of (a) $10^{-3} \mathrm{~s}-1$, (b) $10^{-2} \mathrm{~s}^{-1}$, and (c) $10^{-1} \mathrm{~s}^{-1}$

A 1.12 Compressive flow curves of two distinct reproducible tests for the 2.0 wt. $\%$ CNT 2024Al composite compressed at $400^{\circ} \mathrm{C}$ and strain rates of (a) $10^{-3} \mathrm{~s}-1$, (b) $10^{-2} \mathrm{~s}^{-1}$, and (c) $10^{-1} \mathrm{~s}^{-1}$ 


\section{NOMENCLATURE}

Acronym

$\mathrm{Al}$

AMC

ASTM

BC

bcc

CAFE

Cd

CDRX

$\mathrm{Ce}$

CTE

CVD

CNT

CRSS

$\mathrm{Cu}$

CYS

DMM

DRX

DRY

EDS

EBSD
Definition

Aluminum

Aluminum matrix composite

American Society for Testing of Materials

Band contrast

Body centered cubic

Corporate average fuel economy

Cadmium

Continuous dynamic recrystallization

Cerium

Coefficient of thermal expansion

Chemical vapor deposition

Carbon nanotube

Critical resolved shear stress

Copper

Compressive yield strength

Dynamic material model

Dynamic recrystallization

Dynamic recovery

Energy dispersive X-ray spectroscopy

Electron backscatter diffraction 


\begin{tabular}{|c|c|}
\hline $\mathrm{Fe}$ & Iron \\
\hline FESEM & Field emission scanning electron microscopy \\
\hline fcc & Face centered cubic \\
\hline FSD & Forward scatter detector \\
\hline GB & Grain boundary \\
\hline Gd & Gadolinium \\
\hline GOS & Grain orientation spread \\
\hline HAGB & High angle grain boundary \\
\hline hcp & Hexagonal closed packed \\
\hline HRTEM & High-resolution transmission electron microscopy \\
\hline HT & High temperature \\
\hline IPF & Inverse pole figure \\
\hline $\mathrm{La}$ & Lanthanum \\
\hline LAGB & Low angle grain boundary \\
\hline $\mathrm{LCF}$ & Low cycle fatigue \\
\hline LD & Loading direction \\
\hline MA & Mechanical alloying \\
\hline MDs & Molecular dynamics \\
\hline $\mathrm{Mg}$ & Magnesium \\
\hline $\mathrm{MMC}$ & Metal matrix composite \\
\hline $\mathrm{Mn}$ & Manganese \\
\hline MRD & Multiple random distribution \\
\hline MWCNTs & Multi wall carbon nanotubes \\
\hline
\end{tabular}




\begin{tabular}{|c|c|}
\hline $\mathrm{Nd}$ & Neodymium \\
\hline $\mathrm{Ni}$ & Nickel \\
\hline ODF & Orientation distribution function \\
\hline $\mathrm{OM}$ & Optical microscopy \\
\hline $\mathrm{Pb}$ & Lead \\
\hline PLCC & Pearson linear correlation coefficient \\
\hline PM & Powder metallurgy \\
\hline PSN & Particle simulated nucleation \\
\hline $\mathrm{RD}$ & Rolling direction \\
\hline RE & Rare-earth \\
\hline RPM & Rotation per minute \\
\hline RSS & Resolved shear stress \\
\hline RT & Room temperature \\
\hline $\mathrm{Sc}$ & Scandium \\
\hline SEM & Scanning electron microscope \\
\hline $\mathrm{SiC}$ & Silicon carbide \\
\hline $\mathrm{SiC}_{\mathrm{W}}$ & Silicon carbide whiskers \\
\hline $\mathrm{SiC}_{\mathrm{P}}$ & Silicon carbide particles \\
\hline SRCC & Spearman rank order correlation coefficients \\
\hline TB & Twin boundary \\
\hline TEM & Transmission electron microscopy \\
\hline TD & Transverse direction \\
\hline TDs & Twinning dislocations \\
\hline
\end{tabular}




$\begin{array}{ll}\text { TEM } & \text { Transmission electron microscope } \\ \text { TYS } & \text { Tensile yield strength } \\ \text { USAMP } & \text { United States Automotive Materials Partnership } \\ \text { UTS } & \text { Ultimate tensile strength } \\ \text { XRD } & \text { X-ray diffraction } \\ \text { Y } & \text { Yttrium } \\ \text { YS } & \text { Yield strength } \\ \text { Zn } & \text { Zinc } \\ \text { Zr } & \text { Zirconium }\end{array}$

\section{Symbol Definition}

Bragg diffraction angle

$\begin{array}{ll}A, A_{1}, A_{2} & \text { Material constants } \\ a_{0} & \text { lattice parameter } \\ B & \text { Diffraction peak width } \\ b & \text { Fatigue strength exponent } \\ c & \text { Fatigue ductility exponent } \\ d_{\mathrm{g}} & \text { Average grain size } \\ d & \text { Interplanar spacing } \\ e & \text { Eccentricity } \\ E & \text { Young's modulus or modulus of elasticity } \\ \varepsilon & \text { Strain }\end{array}$




\begin{tabular}{|c|c|}
\hline$\varepsilon_{\max }$ & Maximum strain \\
\hline$\dot{\varepsilon}$ & Strain rate \\
\hline$\varepsilon_{f}^{\prime}$ & Fatigue ductility coefficient \\
\hline$\xi(\dot{\varepsilon})$ & Instability parameter \\
\hline$G$ & Power dissipated by plastic deformation \\
\hline$J$ & Dissipation through microstructure evolution \\
\hline K & Strength coefficient \\
\hline$K^{\prime}$ & Cyclic strength coefficient \\
\hline$m$ & Strain rate sensitivity of the material \\
\hline$N$ & Number of cycles \\
\hline$N_{f}$ & Number of cycles to failure \\
\hline$n^{\prime}$ & Cyclic strain hardening exponent \\
\hline$\eta$ & Efficiency of power dissipation \\
\hline$P_{z}$ & Pinning force \\
\hline$Q$ & Activation energy for hot deformation \\
\hline$r$ & Particle radius \\
\hline$R$ & Universal gas constant \\
\hline$R_{\varepsilon}$ & Strain ratio \\
\hline$T$ & Deformation temperature \\
\hline$V_{1}, V_{2}, V_{3}$ & Twin variants \\
\hline$V_{p}$ & Particle volume fraction \\
\hline$X$ & Input factor \\
\hline
\end{tabular}




\begin{tabular}{|c|c|}
\hline$\tilde{X}$ & Scaled input factor \\
\hline$\sigma$ & Stress \\
\hline$\sigma_{U T S}$ & Ultimate tensile stress \\
\hline$\sigma_{\max }$ & Peak tensile stress \\
\hline$\sigma_{f}^{\prime}$ & Fatigue strength coefficient \\
\hline$\frac{\Delta \varepsilon_{t}}{2}$ & Total strain amplitude \\
\hline$\frac{\Delta \varepsilon_{e}}{2}$ & Elastic strain amplitude \\
\hline$\frac{\Delta \varepsilon_{\mathrm{p}}}{2}$ & Plastic strain amplitude \\
\hline$\Delta \alpha$ & Angle deviation \\
\hline$\Delta \varepsilon_{t}$ & Total strain range \\
\hline$\Delta \varepsilon_{e-t}$ & Tensile elastic strain range \\
\hline$\Delta \varepsilon_{e-c}$ & Compressive elastic strain range \\
\hline$\Delta \varepsilon_{a n}$ & Anelastic strain range \\
\hline$\Delta \varepsilon_{a n-t}$ & Tensile anelastic strain range \\
\hline$\Delta \varepsilon_{a n-c}$ & Compressive anelastic strain range \\
\hline$\frac{\Delta d}{d}$ & Systematic error \\
\hline$\gamma$ & Grain boundary energy \\
\hline$\alpha$ & Stress multiplier \\
\hline$\lambda$ & wavelength of the radiation \\
\hline$\varphi_{1} \Phi \varphi_{2}$ & Euler angles \\
\hline
\end{tabular}




\section{CHAPTER 1}

\section{INTRODUCTION}

\subsection{Background and motivation}

Because of the huge environmental concerns and mounting global energy demands in the transportation industry, vehicle lightweighting has been recently recognized as one of the most important methods to improve fuel efficiency and reduce anthropogenic climate-changing and human death-causing ${ }^{1}$ emissions [1-5]. Passenger vehicles, usually thought as the least detrimental ones, are reported to be among the most abundant and diverse means of transportation [6,7]. Hence, finding alternatives to reduce their fuel consumption has been of major interest, attracting a great deal of research curiosity, especially since fuel efficiency was found to be ameliorated by $\sim 6-8 \%$ for each $10 \%$ weight reduction [8]. In this context, lightweight alloys such as $\mathrm{Mg}$ and $\mathrm{Al}$ have been of tremendous interest as well as factors mainly affecting the enhancement of their mechanical properties, such as rare earth (RE) microalloying and carbon nanotube (CNT) reinforcement.

Mg alloys represent attractive alternatives because of their low density, high strength-to-weight ratio [9], and superior damping capacity [10]. Nevertheless, several constraints are faced in their applications, including high directional anisotropy and poor formability at room temperature

\footnotetext{
${ }^{1}$ According to Science News entitled "Air pollution kills 7 million people a year" on March 25, 2014 at http://www.sciencemag.org/news/sifter/air-pollution-kills-7-million-people-year: “Air pollution isn't just harming Earth; it's hurting us, too. Startling new numbers released by the World Health Organization today reveal that one in eight deaths are a result of exposure to air pollution. The data reveal a strong link between the tiny particles that we breathe into our lungs and the illnesses they can lead to, including stroke, heart attack, lung cancer, and chronic obstructive pulmonary disease."
} 
(RT). The development of strong preferred orientation (or texture) in the deformation process leads to the tension-compression yield asymmetry and mechanical anisotropy in the wrought $\mathrm{Mg}$ alloys [11], due to the hcp crystal structure and the limited deformation modes [12]. Both tension-compression yield asymmetry and RT formability could be effectively improved via alloy composition adjustment, by adding RE elements [13] due to their tendency to induce texture randomization during hot processes, leading to the decrease of texture intensities [14]. $\mathrm{RE}$ elements were also reported to alternate the bonding energy between $\mathrm{Mg}$ and $\mathrm{RE}$ atoms, thus increasing the possibility of non-basal slip and inhibiting deformation twinning [15]. Detailed information of such RE-Mg alloys under dynamic cyclic loading condition is still lacking and it is unclear how RE-elements impact the tensile-compressive yield asymmetry generally occurring in RE-free extruded Mg alloys, and to what extent the RE addition would affect the fatigue life.

Lightweight automotive applications have also been relying on $\mathrm{Al}$ alloys. For instance, powertrain components require alloys with high thermal stability such as $\mathrm{Al}$ and there is a continuous need to increase their service temperature limit [16]. Therefore, to maintain the desired strength of the alloy at increased temperatures, aluminum matrix composites (AMCs) are recognized for their superior mechanical properties. CNTs are considered as attractive reinforcements for AMCs due to their excellent mechanical and physical properties as well as light weight $[17,18]$. However, there are still several challenging aspects that need to be resolved including interfacial reactions, low wettability, and anisotropic properties [19]. Mechanical testing at various temperatures is required to establish the structure-property relationships of the composites; however, most of the studies in the literature [20-22] involved the tensile behavior of the CNT/Al composites at RT. In addition, nanocomposites and other structural materials 
could be subjected to either tensile or compressive forces at varying temperatures when used in systems and devices. A few attempts were published in this matter, for instance, the sensitivity of CNTs to buckling and kinking, under compression, was studied by Silvestre et al. [22] using molecular dynamics (MDs). A few other studies on the compressive behavior of CNT reinforced composites indicated the beneficial role of CNT additions [23-25]. For instance, Bakshi et al. [24] observed a significant improvement in the compressive yield strength (CYS) by $27 \%$ and $77 \%$ at RT through the addition of 5.0 and 10.0 wt.\% CNTs, respectively. It is, however, uncertain whether this improvement would sustain at $\mathrm{HT}$ and exactly how the compressive behavior of CNT reinforced composites would change with deformation temperature. While some limited microstructural studies on the CNT reinforced $\mathrm{Al}$ composite were reported in the literature [26-28], 3-D electron backscatter diffraction (EBSD) investigations are not available, to the authors' knowledge. Compressive micro-strains were also noted in the CNT/Al composites [28-30], resulting in X-ray diffraction (XRD) peak broadening, however no actual quantification of the resultant lattice change is reported. It is hence necessary to understand how the addition of CNTs affects the lattice change, microstructure and texture, and especially what strengthening mechanisms are involved.

Considering all these aspects mentioned above, with respect to the performance enhancement of $\mathrm{Mg}$ and $\mathrm{Al}$, being two of the most attractive lightweight structural alloys, the proposed work starts by investigating the deformation behavior of RE-free wrought Mg alloys, through RT compression tests of an extruded AZ31 Mg alloy. Then, a recently-developed ZEK100 Mg alloy with a low RE content (i.e., $0.2 \mathrm{wt} . \% \mathrm{Nd}$ ) is subjected to strain-controlled push-pull type fatigue tests at a constant strain rate and RT, while being compared to RE-free and high RE-containing 
alloys. Both cyclic and anelastic behavior were evaluated. Effect of CNT addition is studied by RT and HT compression tests on a 2024Al alloy and a $2.0 \mathrm{wt} . \%$ CNT/2024Al composite.

\section{Objectives and scope of the dissertation}

The overall purpose of the proposed research is to identify the deformation mechanisms and analyze the key factors affecting the mechanical properties of lightweight $\mathrm{Mg}$ and $\mathrm{Al}$ alloys. The

choice of tests (e.g., tensile, compressive, fatigue) in each investigation is dependent on the amount and shape under which the material was received (e.g., sheet, bar, rod). For Mg alloys, to identify the beneficial effect of RE-elements is the purpose of the present studies, whereas for the $\mathrm{Al}$ alloys, the impact of CNT reinforcement in strengthening Al alloys is the main target. The reason behind both $\mathrm{Mg}$ and $\mathrm{Al}$ alloys comes from the multi-material concept to achieve vehicles with maximal lightweight potential, which are more fuel efficient and less detrimental environmentally. Tis could be obtained by overcoming some of the drawbacks of conventional $\mathrm{Mg}$ and $\mathrm{Al}$ alloys via adding a small amount of RE elements and CNTs. These objectives were realized through the three main investigations detailed below along with the specified tasks.

$\rightarrow$ Investigation 1: Gain a clear understanding of the major deformation behavior of RE free wrought Mg alloys at RT, i.e., deformation twinning.

- Task 1: Study the deformation twinning evolution through step-by-step compressive deformation under various strain amounts.

- Task 2: Identify and model main features of single and double twin nucleation, growth and interactions. 
$\rightarrow$ Investigation 2: Identify the impact of RE microalloying on the deformation behavior of wrought Mg alloys.

- Task 1: Conduct a comparative study between the deformation behavior of RE-free and low RE-containing Mg alloys.

- Task 2: Study the effect of RE-elements on texture weakening and randomization as well as morphological changes of the microstructural features.

- Task 3: Observe the effect of RE elements on the cyclic deformation characteristics and fatigue life.

- Task 4: Characterize the effect of RE on the pseudo-elastic (i.e., anelastic) behavior.

$\rightarrow$ Investigation 3: Evaluate the effect of CNT reinforcement on the deformation behavior, activation energy and hot workability of Al alloys.

- Task 1: Identify deformation and strengthening mechanisms of CNT reinforced AMCs.

- Task 2: Characterize the hot deformation in CNT reinforced AMCs compared to Al base alloys using activation energy.

- Task 3: Determine the optimized hot workability window of CNT reinforced AMCs using 3D processing maps.

\section{Structure of the dissertation}

The presented dissertation has been structured as follows:

Chapter 2 - A generalized literature survey on some prior research development on $\mathrm{Mg}$ and $\mathrm{Al}$ is presented, where the material characteristics, effects of microalloying and reinforcements, and deformation behavior are reviewed. 
Chapter 3 - Materials and experimental procedures used in this study are detailed.

Chapter 4 - A comprehensive study of the twinning behavior of a RE-free AZ31 Mg alloy with identification and modeling of major scenarios of single and double twin nucleation, growth and interactions is presented.

Chapter 5 - Effect of RE elements on the deformation behavior of rolled RE-containing ZEK100 alloy through the study of the strain-controlled low cycle fatigue properties is described. Chapter 6 - A more focused study on the effect of strain ratio on the cyclic deformation and anelastic behavior of ZEK100 is discussed.

Chapter 7 - Deformation and strengthening mechanisms of the 2.0 wt.\% CNT 2024Al composite in comparison with the 2024Al base alloy through hot compression are identified.

Chapter 8 - The hot deformation in the CNT reinforced composite and the base alloy using activation energy and approximation of the optimized hot workability regions using the 3-D processing maps is characterized.

Chapter 9 - Conclusions, major contribution and future scope of the present study are summarized. 


\section{CHAPTER 2}

\section{LITERATURE REVIEW}

In this section, a brief literature review is presented to facilitate a concise overview of the deformation behavior of $\mathrm{Mg}$ and $\mathrm{Al}$ alloys with and without respective $\mathrm{RE}$ and $\mathrm{CNT}$ additions, and to uncover areas where further research is required.

\subsection{History and development in Mg and Al alloys}

Since its early discovery back in $1774, \mathrm{Mg}$ was found to be the $6^{\text {th }}$ most abundant element, constituting $2 \%$ of the total mass of the Earth's crust [31]. It can be produced from seawater, brines and Mg-bearing minerals which give unlimited supply of ore reserves. Based on the current world's use levels, it was estimated that there would be enough Mg in the Dead Sea for at least 22,000 years [32]. $\mathrm{Mg}$ is the lightest structural metallic material available (with a density of $1.74 \mathrm{~g} / \mathrm{cm}^{3}, \sim 36 \%$ less than aluminum, $\sim 4.5$ times less than steel and nearly the same as many polymers). The melting point and specific heat of $\mathrm{Mg}$ are very close to those of $\mathrm{Al}$, while Young's modulus and shear modulus are about two-thirds of Al [33]. Pure Mg has higher damping capacities than cast iron, in addition to its favorable electromagnetic shielding, dimensional stability, good machinability and recyclability [31]. Mg and its alloys have revived to attract a great deal of research curiosity since 1993. The last decade has witnessed a growing demand for its development in the automotive and other household and sport applications due to its large potential for weight saving [34]. However, the use of $\mathrm{Mg}$ as a structural material is still limited and it is mainly used as an alloying element in other alloys. For instance, alloying $\mathrm{Mg}$ 
with aluminum, manganese, rare-earth, thorium, zinc or zirconium increases the strength to weight ratio of the alloys [35]. A report released by USAMP (United States Automotive Materials Partnership) in 2006, implied the need of increasing the use of Mg alloys by $340 \mathrm{lbs}$ per car by the year of 2020 , leading to $\sim 15 \%$ of the vehicle weight reduction [36], which has been the target of auto industries for the last decade.

As for $\mathrm{Al}$, it has been discovered back in the year of 1808 and started to be effectively produced after 17 years, making the history of $\mathrm{Al}$ short in comparison with metals like iron and copper which have been used for thousands of years [37]. However, Al has become increasingly popular due to its low density and high-strength-to-weight ratio which distinguished it from the other metals. Al is also characterized by its high corrosion resistance, good ductility; thermal and electrical conductivity and convenient surface finish [38]. Al has been appreciated more and more by auto industries. In fact, in a 2013 press release at WWJ Auto Summit, vehicle lightweighting was regarded as the auto industry's best opportunity to achieve CAFE (Corporate Average Fuel Economy) standard and it was also stated that by maximizing the use of $\mathrm{Al}$ and its alloys, along with modern manufacturing technologies, the mass of every vehicle could be reduced by more than $95 \mathrm{~kg}$. Hence, the application of $\mathrm{Al}$ alloys could result in effective weight reduction and energy-saving, leading to a better environmental protection.

Table 2.1 displays the physical properties of $\mathrm{Mg}$ and $\mathrm{Al}$ in comparison with other structural materials. The lower densities of both materials was obvious, with the density of $\mathrm{Mg}$ being less than two-thirds of that of $\mathrm{Al}$ while the melting point and specific heat are almost the same for both materials. The Young's modulus and shear modulus of $\mathrm{Mg}$ are about two-thirds of those of 
Al. Both pure $\mathrm{Mg}$ and $\mathrm{Al}$ alloys have been reported in [39] to have relatively low yield stress (YS) and ultimate tensile stress (UTS), therefore they are usually alloyed and/or reinforced with the adequate elements to achieve the needed mechanical properties.

Table 2.1 Physical properties of the materials used in this study compared to others [39].

\begin{tabular}{|c|c|c|c|c|c|}
\hline & Mg & Al & Ti & Fe & Cu \\
\hline Lattice structure & HCP & FCC & HCP & BCC & FCC \\
\hline Density $\left(\mathrm{g} / \mathrm{cm}^{3}\right)$ & 1.74 & 2.70 & 4.51 & 7.87 & 8.98 \\
\hline Melting point $\left({ }^{\circ} \mathrm{C}\right)$ & 650 & 660 & 1668 & 1535 & 1083 \\
\hline $\begin{array}{c}\text { Specific heat } \\
\left(\mathrm{J} / \mathrm{m}^{\circ} \mathrm{C}\right)\end{array}$ & 0.24 & 0.22 & 0.13 & 0.11 & 0.09 \\
\hline $\begin{array}{c}\text { Young's modulus } \\
(\mathrm{GPa})\end{array}$ & 45 & 76 & 114 & 190 & 136 \\
\hline $\begin{array}{c}\text { Shear modulus } \\
(\mathrm{GPa})\end{array}$ & 17 & 26 & 45 & 80 & 44 \\
\hline $\begin{array}{c}\text { Thermal } \\
\text { conductivity } \\
(\mathrm{W} / \mathrm{m} . \mathrm{K})\end{array}$ & 167 & 238 & 16 & 73 & 394 \\
\hline
\end{tabular}

Based on their fabrication methods, $\mathrm{Mg}$ and $\mathrm{Al}$ alloys could be classified under two major groups: wrought and cast alloys [40]. Wrought alloys are usually shaped by means of plastic deformation (e.g., rolling, extruding, forging, and drawing) which leads to an important reduction in the grain size of the microstructure and in removing any defects generated during casting. Mechanical properties of the wrought alloy are hence highly improved. On the other hand, casting of the alloy into its final shape is still more economical; however, any defect that might occur in the casting process would be carried over into the final product. Minimizing this type of defects is primordial in applications requiring mechanical performance, mainly fatigue resistance [41]. 


\subsection{Deformation systems in $\mathrm{Mg}$ alloys}

As stated above, Mg has a hcp crystal structure, displayed in Fig.2.1(a) along with the position of the atoms. Possible basal slip directions are also provided in Fig.2.1(b). The cell in the hcp structure can be geometrically identified by four axes: Three coplanar axes $\mathrm{a}_{1} \mathrm{a}_{2} \mathrm{a}_{3}$, and the $c$ axis, which is perpendicular to the basal plane containing $a_{1}, a_{2}$, and $a_{3}$. The c/a ratio is considered as an important parameter in determining the deformation behavior, where $a$ is the interatomic distance in the basal plane along any of the three directions displayed in Fig.2.1(b), and $c$ is the distance between the first and third plane in Fig.2.1(a).

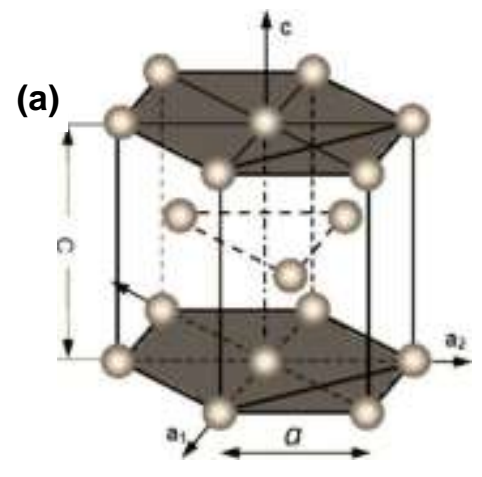

(b)

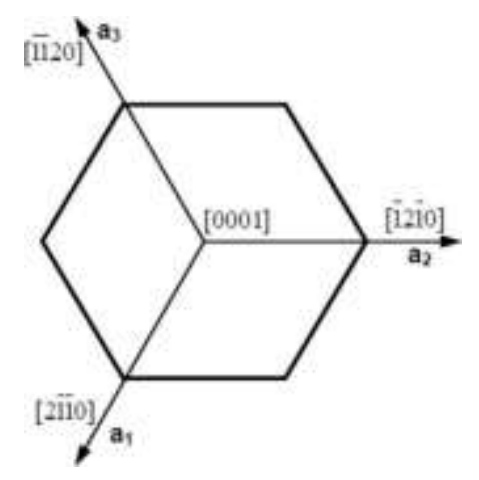

Fig.2.1 (a) Hexagonal close packed (hcp) crystal structure, and (b) slip directions [42].

For pure $\mathrm{Mg}$, lattice parameters at RT are $a=0.32092 \mathrm{~nm}$ and $c=0.52105 \mathrm{~nm}$ [43], leading to a c/a ratio of 1.6236, close to the ideal value of 1.633 [42]. When a compressive load is applied along the $c$-axis, and depending on the $c / a$ ratio, some hexagonal close packed (HCP) metals (e.g., $\mathrm{Zr}$ and $\mathrm{Cd}$ ) show twinning dominant deformation. However, in the other hand, a tensile load is needed along the $c$-axis for twinning to happen since it is the profuse extension twinning direction where basal planes are initially perpendicular to the tensile loading. 


\subsubsection{Dislocation slip systems}

Polycrystalline materials can accommodate deformation by the activation of five independent slip systems [44]. The most common slip systems that could be activated for Mg are displayed in Fig.2.2(a), (b), (c) and (d) and summarized in Table 2.2. Among these four dominant slip systems, three involve the slip dislocations with $\langle 11 \overline{2} 0\rangle$ (i.e., $\langle a\rangle$ type vectors). Based on the von Mises criterion, basal and prismatic slip can only offer two independent slip modes in each case.

(a)

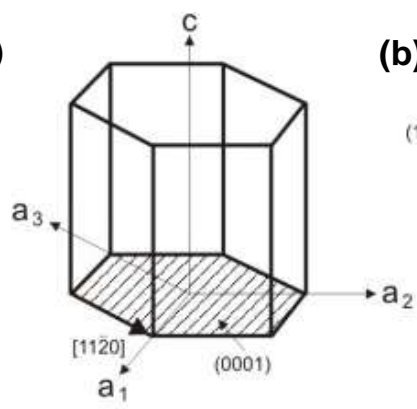

$(0001)<1120>$ (b)

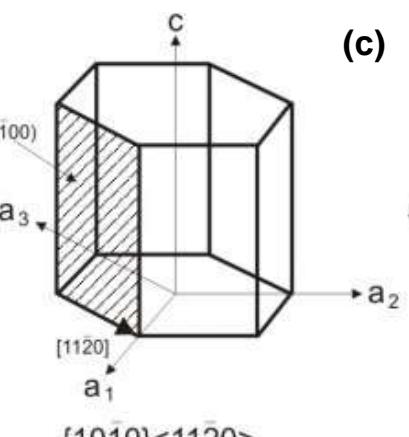

$\{1010\}<1120>$

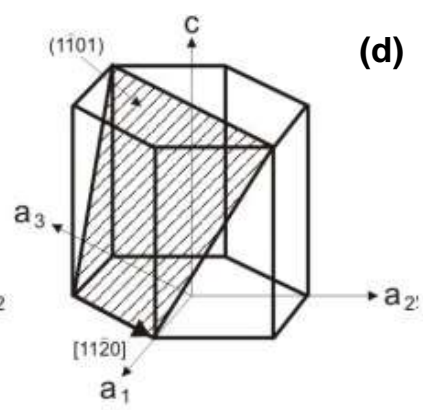

$\{1011\}<1120>$

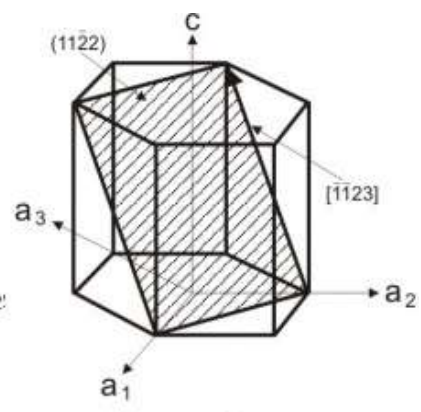

$\{1122\}<1123>$

Fig.2.2 (a) Basal- $\langle a\rangle$, (b) prismatic- $\langle a\rangle$, (c) pyramidal- $\langle a\rangle$, and (d) pyramidal- $\langle$ c $+a\rangle$ slip systems in $\mathrm{Mg}$ [44].

Table 2.2 Independent slip systems in Mg [45].

\begin{tabular}{cccc}
\hline Slip system & Direction & $\begin{array}{c}\text { Crystallographic } \\
\text { component }\end{array}$ & $\begin{array}{c}\text { Number of } \\
\text { independent modes }\end{array}$ \\
\hline Basal slip & $a$ & $\{0001\}-\{11 \overline{2} 0\}$ & 2 \\
Prismatic slip & $a$ & $\{10 \overline{1} 0\}-\{11 \overline{2} 0\}$ & 4 \\
$\mathbf{1}^{\text {st }}$ order pyramidal slip & $a$ & $\{10 \overline{1} 1\}-\{11 \overline{2} 0\}$ & 5 \\
$\mathbf{2}^{\text {nd }}$ order pyramidal slip & $c+a$ & $\{11 \overline{2} 2\}-\{11 \overline{2} 3\}$ & \\
\hline
\end{tabular}


While the $1^{\text {st }}$ order pyramidal slip can offer four independent slip modes, it is still short of the required five for homogeneous deformation. Only the $2^{\text {nd }}$ order pyramidal slip, with its non-basal vector (or $\langle c+a\rangle$ ), will accommodate deformation along the $c$-axis and meet the requirement for five independent slip modes. However, this type of dislocation slip does not normally occur at RT.

\subsubsection{Deformation twinning}

Overall, the concept of twinning is coming from the fact that a shear force can produce atomic displacements such that on one side of a plane (i.e., the twin boundary), atoms are located in mirror image positions of atoms on the other side [46]. The plane of symmetry between the two portions is known as the twinning plane. The typical atomic arrangement of twinning is illustrated in Fig.2.3(a) and final atom positions are displayed in Fig.2.3(b). Twinning occurs in magnesium due to its hcp crystal structure with low crystal symmetry which leads to insufficient number of slip systems at RT [47].
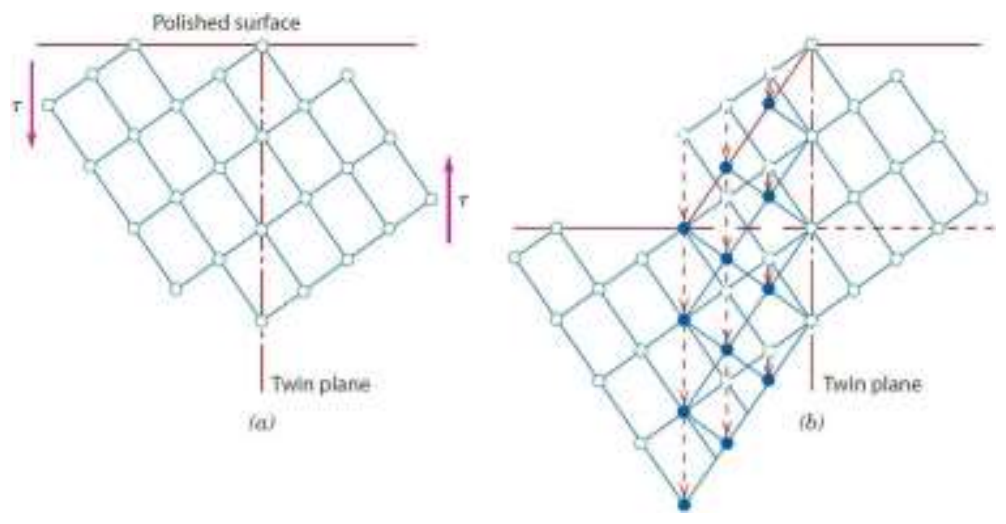

Fig.2.3 (a) Schematic diagram showing how twinning results from an applied shear stress $\tau$, and (b) open circles represent atoms that did not change position; dashed and solid circles represent original and final atom positions, respectively [44]. 
Twinning was the subject of many investigations in the literature. Park et al. [48] studied the twinning behavior of rolled $\mathrm{Mg}$ alloy subjected to consecutive in-plane compressions along two orthogonal directions using EBSD. Specimens were first compressed up to 6\% along the rolling direction (RD), and then further compressed to 3 and $6 \%$ along the transverse direction (TD).

The result exhibited various twinning modes. Extension twinning $\{10 \overline{1} 2\}$ was first observed in residual parent grain, followed by $\{10 \overline{1} 2\}-\{10 \overline{1} 2\}$ double twinning in twin bands. Precompression effect on plastic deformation behavior of AZ31 Mg alloy has also been observed by Xin et al. [49]. Gharghouri et al. [50] studied the mechanical properties of an extruded Mg-7.7 at.\% Al alloy by means of in-situ neutron diffraction. The occurrence of twinning was easily detected, considering that it would lead to an abrupt reorientation of the crystal lattice, reflected by a change in the peak density. Two types of twinning are observed in $\mathrm{Mg}$ alloys, $\{10 \overline{1} 2\}\langle\overline{1} 011\rangle$ extension twins, and $\{10 \overline{1} 1\}\langle\overline{1} 012\rangle$ contraction twins [51]. Extension twins were mainly investigated in the literature for Mg alloys. For instance, El Kadiri et al. [52] conducted EBSD serial image analyses on an AM30 alloy compressed under profuse $\{10 \overline{1} 2\}$ twinning conditions at different strain levels. They proved that twin nucleation and twin propagation rates strongly depend on the number of activated twins in a given grain.

\subsubsection{Twin growth}

Nucleation of twins is promoted in the stress-concentrated regions such as grain boundaries and dislocation sites. Once a twin is formed, it propagates when the resolved shear stress (RSS) on the twinning system surpasses the critical resolved shear stress (CRSS) for twin growth, which is usually less than the CRSS for twin nucleation [53]. Twin growth is achieved through the gliding 
of twinning dislocations (TDs) in the matrix and on the twin boundary (TB), where the driving force is the stress acting on TDs at the TB interface. Two phenomena could be attributed to twin growth [53]: matrix reduction and twin propagation which are activated based on the average stresses in the matrix and in the twin. Matrix reduction occurs due to the migration of twin boundaries induced by the RSS in the matrix and thus reduces the current volume of the matrix and increases the volume and thickness of the twin once they merge after twin boundary disappearance (i.e., twin boundary coalescence).

\subsubsection{Double twinning}

Double twinning could be simply defined as the activation of a twin within a pre-existing twin under a similar or a different variant [54]. Several investigations were conducted on double twinning in the literature. Ma et al. [55] studied twinning in AM30 Mg alloy under profuse $\{10 \overline{1} 2\}$ twinning conditions and reported double twin nucleation, mainly $\{10 \overline{1} 1\}-\{10 \overline{1} 2\}$, where the preliminary parent twin is the contraction twin and the secondary is the extension twin, and $\{10 \overline{1} 3\}-\{10 \overline{1} 2\}$ which they found to play an important role in the sample fracture.

Beyerlein et al. [54] proposed a dislocation mechanism for the formation of $\{10 \overline{1} 1\}-\{10 \overline{1} 2\}$ double twin structures in hcp crystals through the nucleation of secondary twins within primary twin domains, and stated that the secondary twins associated with the most commonly observed double twin variants nucleate and thicken by a sequence of three distinct dissociation reactions of mixed basal dislocations. 


\subsection{RE microalloying in $\mathrm{Mg}$ alloys}

\subsubsection{Brief history of RE elements}

RE elements represent the set of seventeen chemical elements in the periodic table which include the fifteen lanthanides as well as scandium $(\mathrm{Sc})$ and yttrium $(\mathrm{Y})$. The designation of these elements as "rare earth" comes from the fact that most of them were isolated in the $18^{\text {th }}$ and $19^{\text {th }}$ century as oxides from rare minerals, and because of their reactivity, RE elements were found to be difficult to refine to pure metals [56]. Efficient separation processes for RE elements were not developed until the $20^{\text {th }}$ century, when all elements were identified. Most RE elements are not as uncommon in nature as their names imply. In fact, cerium (Ce) comprises more of earth's crust than copper $(\mathrm{Cu})$ or lead $(\mathrm{Pb})[57]$.

\subsubsection{Major effects of RE elements on Mg alloys}

Plastic anisotropy is greatly influenced by the crystallographic texture formed in $\mathrm{Mg}$ alloys. Usually, wrought $\mathrm{Mg}$ alloys display a strong texture with most of the basal planes aligned parallel to rolling direction (RD) or extrusion direction (ED) $[58,59]$. However, it has been proven that RE alloying elements can modify the contribution of various deformation modes to the overall deformation behavior of the alloy, leading to the variation of texture. Earlier studies were started by Ball and Prangnell [60] who reported a weaker and randomized texture for the extruded WE54 Mg alloy, characterized by a high content of RE elements (5.2 wt.\% Y, 1.74 wt.\% $\mathrm{Nd}$, and 0.95 wt.\% Ce and La). Texture weakening is mainly attributed to the recrystallization 
which is highly affected by the presence of RE elements. Mackenzie and Pekguleryuz [61] studied the Mg-Zn-Ce alloys and reported a non-basal texture component along the transverse direction (TD) in the shear bands that evolved during rolling, which became dominant in the fully recrystallized microstructure. Senn and Agnew [62] focused on the texture randomization of RE-containing Mg alloys during hot deformation and discussed the presence of shear bands as a result of plastic deformation, where some grain nuclei (i.e., growing into the bands) exhibited a highly randomized texture compared to the surrounding non-recrystallized zone.

\subsubsection{Microstructure of RE-containing wrought Mg alloys}

Typical optical micrographs of hot-extruded Mg-8Gd-xZn-0.4Zr, Mg-8Gd-Zn-0.4Zr, and Mg8Gd-3Zn-0.4Zr alloys [63] are represented in in Fig.2.4(a), (b), and (c), respectively.
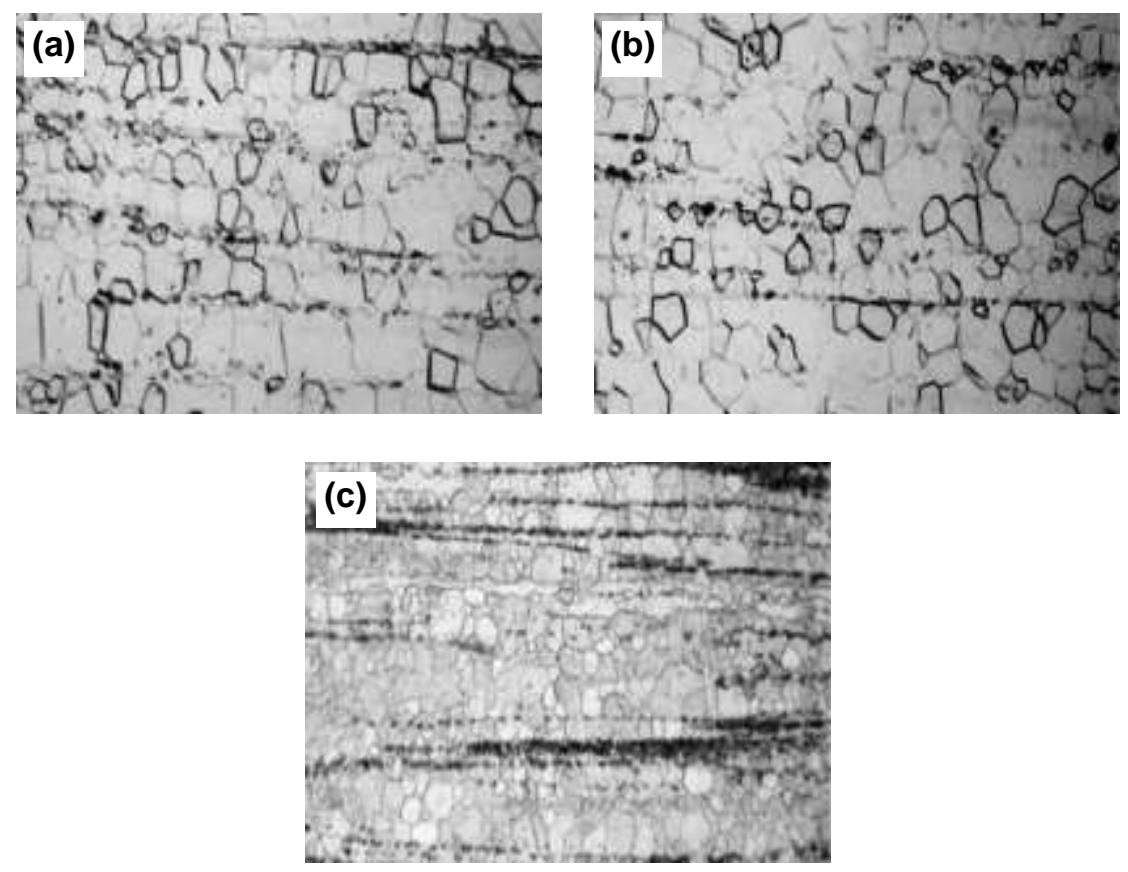

Fig.2.4 Optical images of the as-extruded specimens of (a) Mg-8Gd-0.4Zr alloy, (b) Mg-8Gd1Zn-0.4Zr alloy, and (c) Mg-8Gd-3Zn-0.4Zr alloy [63]. 
It can be seen from Fig.2.4 that for the three presented alloys, the Gd-containing intermetallic compounds (i.e., second-phase particles) were broken during hot extrusion, and then dispersed along one specific direction (i.e., extrusion direction). Average recrystallized grain sizes were reported for each of the alloys as 8, 6, and $3 \mu \mathrm{m}$ for the $\mathrm{Mg}-8 \mathrm{Gd}-0.4 \mathrm{Zr}, \mathrm{Mg}-8 \mathrm{Gd}-1 \mathrm{Zn}-0.4 \mathrm{Zr}$, and Mg-8Gd-3Zn-0.4Zr alloys, respectively, indicating the combined effect of RE and $\mathrm{Zr}$ elements along with increasing $\mathrm{Zn}$ content. In fact, due to the plastic deformation induced during hot extrusion, recrystallized grains were observed to form along grain boundaries, which led to the accumulation of dislocations and the occurrence of the dynamic recrystallization (DRX) process [63]. Similar extruded microstructures were also reported by Liu et al. in [64]. The previous could mainly be linked to our investigations on the rolled ZEK100 Mg alloy where almost fully recrystallized and equiaxed grains were observed after rolling and after annealing to remove the cold working effects.

\subsubsection{Crystallographic texture of RE-containing wrought Mg alloys}

Wrought Mg alloys usually exhibit strong basal textures with basal planes of most grains aligned parallel to rolling or extrusion directions [65]. However, as mentioned before, it has been acknowledged that when $\mathrm{Mg}$ is alloyed with RE elements, it can develop weaker textures than the conventional $\mathrm{Mg}$ alloys [60]. In fact, a number of RE elements were considered as highly effective texture modifiers such as Gd [66], Y [67], Ce [11], La [68], and Nd [69,70]. Even for low alloying concentrations, a change was observed in the preferred orientations for the RE-Mg alloys from $<10 \overline{1} 0>$ to new positions such as $<11 \overline{2} 1>,<11 \overline{2} 2>$ or $<20 \overline{2} 1>$, referred to as RE texture components [71]. 
Fig.2.5 displays the evolution of texture intensities in an Mg-Gd binary alloy [11]. Major texture weakening occurred within the first 1 wt.\% Gd addition, after which the change of texture became less pronounced. Besides, RE texture components are well oriented for basal slip when tested in an appropriate orientation, which results in a substantial gain of ductility and a reduction of the tension-compression asymmetry observed in the conventional wrought $\mathrm{Mg}$ alloys [65]. Likewise, Bohlen et al. [67] reported that the textures of wrought RE-containing Mg alloys are usually characterized by a greater tilt of basal poles towards the transverse direction (TD), rather than the rolling (or extrusion) direction (RD) which stimulates the activation of basal slip during loading in TD rather than RD. Similar observation will be made when studying the crystallographic texture of the Nd containing ZEK100 Mg alloy in the coming chapters.

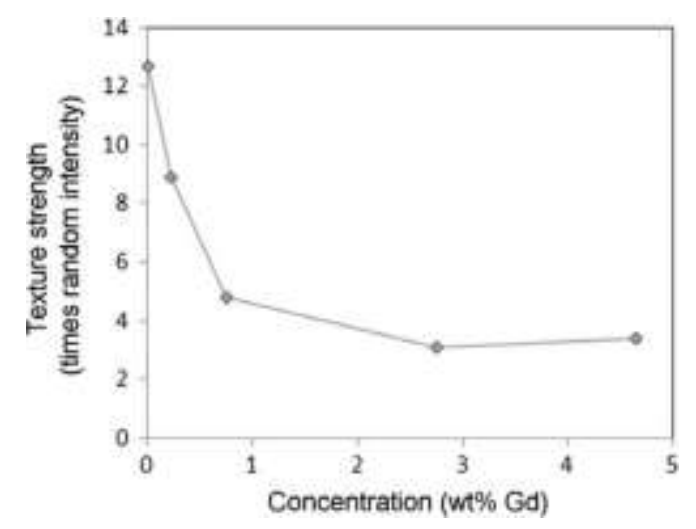

Fig.2.5 Effect of Gd content on the texture intensities of an Mg-Gd alloy [11].

\subsubsection{Fatigue and anelastic behavior of RE-Mg alloys}

As emphasized above, RE-containing Mg alloys are being considered for lightweight automotive applications and the design of structural components require strain-controlled fatigue behavior, since they undergo dynamic loading in service [72]. Some studies on the fatigue of RE- 
containing $\mathrm{Mg}$ alloys have been reported in the literature. For instance, Yang et al. [73] conducted very high cyclic fatigue tests of a RE-containing extruded Mg alloy (Mg-12Gd-3Y$0.5 \mathrm{Zr}$,) and observed a highly reduced tension-compression yield asymmetry and enhanced fatigue failure resistance. The fatigue strength of extruded $\mathrm{Mg}-10 \mathrm{Gd}-1 \mathrm{Nd}$ and $\mathrm{Mg}-10 \mathrm{Gd}$ alloys in the form of S-N curves was also evaluated by Maier et al. via stress-controlled high cycle fatigue tests [74]. A limited number of strain-controlled low cycle fatigue tests on the REcontaining wrought $\mathrm{Mg}$ alloys have been performed recently, including Mg-10Gd-3Y-0.5Zr [75], Mg-3Nd-0.2Zn-0.5Zr [76], Mg-8Gd-3Y-0.5Zr [15], where the basal texture was observed to be weakened and the tension-compression yield asymmetry basically disappeared as reflected by the nearly symmetrical hysteresis loops. However, unlike the case of the face centered cubic (fcc) structures [77], the non-linear anelastic (or pseudo-elastic) behavior persisted. While REcontaining wrought $\mathrm{Mg}$ alloys exhibited an improved fatigue life [74-76], material cost remains one of the most important aspects in the automotive sector, and needs to be affordable for the general public. Thus a RE-free and low Nd-containing (on the order of $\sim 0.2 \mathrm{wt} . \% \mathrm{Nd}$ only) ZEK100 wrought Mg sheet alloy has been the subject of the investigations presented in this dissertation. While tensile loading-unloading tests with an incremental strain were performed [78], no fatigue data on the ZEK100 Mg alloy under strain-controlled tests are available in the literature

\subsection{Low Nd-containing ZEK100 vs. RE-free AZ31}

Microstructure and texture development of ZEK100 alloy have been studied during warm rolling and annealing $[12,14]$. Forming-limit diagrams were also developed at elevated temperatures and 
varying strain rates, and the ZEK100 Mg alloy was observed to reach a true fracture strain, greater by up to $60 \%$ than the AZ31 Mg alloy for all strain paths at all temperatures and strain rates examined [79]. The formability of ZEK100 in a two-stage forming process with intermediate annealing was also studied in [80]. It exhibited superior warm formability over AZ31 alloy, indicating the potential for the volume production of $\mathrm{Mg}$ automotive parts [81]. The tensile properties and constitutive behavior of ZEK100 alloy was also studied at varying strain rates and sample orientations at room temperature (RT) [82]. Basal textures were strongly weakened in comparison with the RE-free AZ31 Mg alloy also considered at RT [83].

\subsection{CNT reinforcement in $\mathrm{Al}$ alloys}

\subsubsection{Brief history of CNT}

Back in 1985, an unplanned experiment resulted in the discovery of a new molecule made purely of carbon. In fact, an infinite class of new molecules has been discovered, referred to as: "the fullerenes" (e.g., $\mathrm{C}_{60}, \mathrm{C}_{70}, \mathrm{C}_{84 \ldots}$ ) [84]. In actuality, however, CNTs had been discovered 30 years earlier without being fully appreciated at that time. In the late 1950s, a "strange" new carbon fiber has been discovered while studying carbon under conditions near its triple point. Straight and hollow tubes of carbon appeared to consist in graphitic layers of carbon separated by the same spacing as the planar layers of graphite [84]. In 1991, after discovery and verification of fullerenes [85], multi-walled nanotubes formed in a carbon arc discharge, and single-walled nanotubes (buckytubes in Fig.2.6) have been identified only two years later. It should be noted 
that no scale bar was shown in Fig.2.6 since it is only indicative of the overall shape of the buckytube as reported in [84].

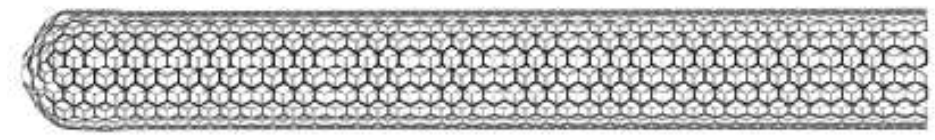

Fig.2.6 A buckytube or a carbon nanotube [84].

\subsubsection{Properties of CNTs}

The mechanical properties of CNTs have been characterized as "extraordinary" in [86] due to their improved stiffness of up to $1000 \mathrm{GPa}$, strength of the order of $100 \mathrm{GPa}$ and thermal conductivity of up to $6000 \mathrm{Wm}^{-1} \mathrm{~K}^{-1}$ [86]. CNTs are attractive reinforcements for $\mathrm{Al}$ matrix composites compared to other carbon materials as listed in Table 2.3.

Table 2.3 Properties of carbon materials [88].

\begin{tabular}{llllll}
\hline Materials & Density $\left(\mathrm{g} / \mathrm{cm}^{3}\right)$ & Strength $(\mathrm{GPa})$ & Modulus $(\mathrm{GPa})$ & $\mathrm{TC}\left(\mathrm{W} \mathrm{m}^{-1} \mathrm{~K}^{-1}\right)$ & $\mathrm{CTE}\left(10^{-6} \mathrm{~K}^{-1}\right)$ \\
\hline PAN-based carbon fibers & $1.75-1.93$ & $2.5-7.0$ & $250-400$ & $8-70$ & $(-0.6)-(-1.1)$ \\
Pitch-based carbon fibers & $2.10-2.19$ & $1.5-3.5$ & $200-800$ & $530-1,100$ & $(-1.3)-(-1.45)$ \\
VGCFs & 2.0 & $2.35-2.9$ & $180-245$ & 1.950 & $1-4$ \\
Crystalline graphite & 2.3 & - & - & $x-y: 3,000$ & $x-y:-1.0$ \\
& & & & $z: 6$ & $z: 29$ \\
Graphite flakes & $2.1-2.3$ & - & - & $\mathrm{a}$ & $\mathrm{a}$ \\
Graphite particles & $2.1-2.3$ & - & - & $\mathrm{b}$ & $\mathrm{b}$ \\
Carbon foams & 0.9 & - & - & $x-y: 70$ & $x-y: 1.02$ \\
& & - & - & $z: 245$ & $z:-1.07$ \\
Diamond & 3.51 & $11-150$ & $270-950$ & $500-3,500$ & $(-10)-(-12)$ \\
CNTs & 2.1 & 130 & 1,002 & $4,840-5,300$ & -1.3 \\
Graphene & - & & &
\end{tabular}


Young's modulus and tensile strength of thinner ropes of aligned MWCNTs have been directly measured in [87] by pulling long ( 2 $\mathrm{mm}$ ) ropes, containing ten thousands of aligned nanotubes (Fig.2.7 [87]) with a specially designed stress-strain puller which can apply an axial force to the rope. Fig.2.8(a) [87] displays the applied total force versus the displacement of the rope. Young's modulus, tensile strength, and elongation were then measured simultaneously and Fig.2.8(b) [87] indicates the tensile strength curve, in which the straight line-segment $\mathrm{A}-\mathrm{B}$ represented the elastic deformation of the sample. The number of the broken tubes in applying load process, was verified by means of the electrical conductivity of the sample (Fig.2.8(c) [87]). And finally, a Young's modulus of 0.46 TPa was obtained as displayed in Fig.2.8(d) [87].

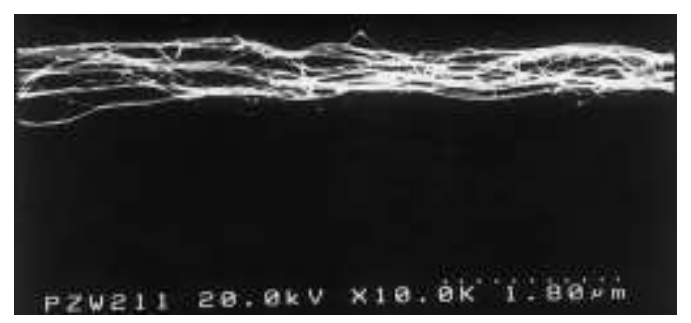

Fig.2.7 SEM image of a nanotube bundle to be pulled for tensile strength experiment [87].

\subsubsection{CNT/Al interface}

Casting and powder metallurgy (PM) has been considered as the major methods for producing CNT/Al composites along with some other novel routes like thermal spray [89] and accumulative roll-bonding [90]. Mechanical interlocking and reaction bonding are the primary bonding types of $\mathrm{CNT} / \mathrm{Al}$ interface [91]. Aluminum carbide $\left(\mathrm{Al}_{4} \mathrm{C}_{3}\right)$ is the main reaction product between CNTs and Al matrix during consolidation due to the high temperature. Since the 
composite studied in this dissertation was fabricated by PM, the features and evolution of $\mathrm{CNT} / \mathrm{Al}$ in PM will be further reviewed below.
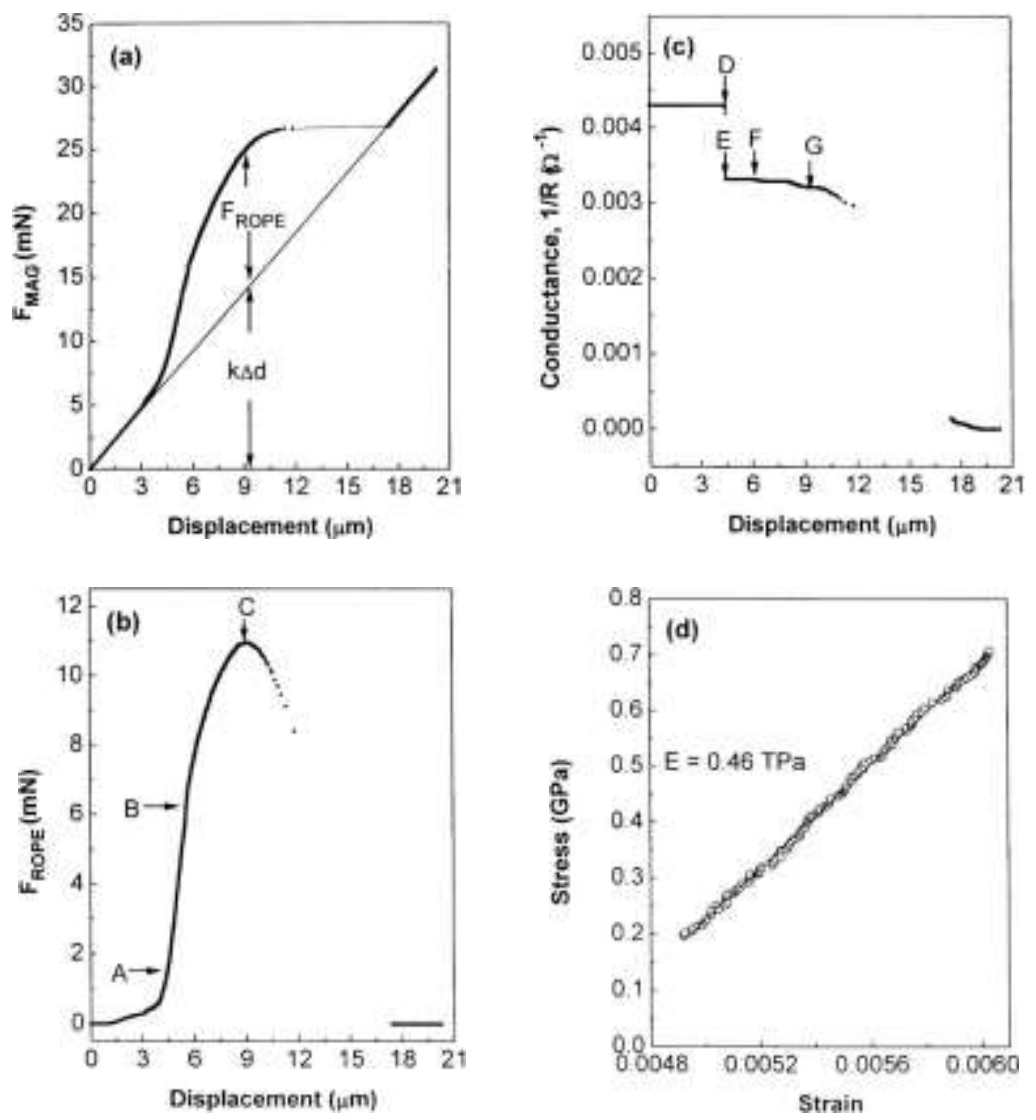

Fig.2.8 (a) Applied total force versus the displacement of the rope, (b) force applied to the nanotube rope versus its displacement, (c) conductance (the inverse of the resistance) of the nanotube rope versus its displacement, and (d) uniaxial stress applied to the nanotube rope versus the strain (elongation) [87].

\subsubsection{CNT evolution in PM}

PM has been greatly considered in producing CNT/Al composites due to its convenience and controllability compared with casting [91]. Preparation of CNT/Al powders and their compact consolidation are the major steps of PM, due to the possibility of CNT agglomeration and the 
compact densification. Both mechanical and chemical methods need to be effective to disperse the CNTs, where applied mechanical forces and chemical modification would help separate tangled CNTs. Various sintering techniques and hot working processes have been used to consolidate the compact. Reaction bonding at CNT/Al interface is established during sintering through diffusion, and the sintered compact is further densified through the elimination of pores by plastic processing [91]. Several studies on the CNT/Al composite powders have been reported in the literature. For instance, Esawi et al. [92] showed a uniform dispersion of CNTs on the Al powder surface with few agglomerations. The unavoidable structural damage of CNTs (i.e., introduced by the severe high energy impact during ball-milling) was pointed by Poirier et al. [93] who studied the mechanically milled CNTs/Al mixture and indicated that after ball-milling, CNTs became shorter and more broken. However, the interfacial bonding of ball-milled CNT/Al interface was still stronger than that achieved by other methods due to the mechanical interlocking [91].

\subsubsection{Functional performance of CNT/Al composite}

Although $\mathrm{C} / \mathrm{Al}$ composites are promising materials in many areas, there are still some challenges related to their functional performance, which need further studies (e.g., interfacial reactions, low wettability, and anisotropic properties) [88]. Michio et al. [94] studied the performance of C/Al composites and identified a few issues related to their use, including the poor wettability, and the reaction between $\mathrm{C}$ and $\mathrm{Al}$ at high temperatures to form the brittle $\mathrm{Al}$ carbide $\mathrm{Al}_{4} \mathrm{C}_{3}$ which strongly decreases the performance of the composites. Liu et al. [95] suggested optimizing the fabrication parameters such as applying an appropriate pressure to promote infiltration, limiting 
the high temperature contact time or using low temperature fabrication methods such as PM. Perez et al. [96] studied CNT/A12024 composites fabricated with a combination of mechanical alloying (MA) and PM routes. Composites were microstructurally and mechanically evaluated in the sintering condition. A homogeneous dispersion of CNTs in the Al matrix was observed by field emission scanning electron microscopy (FESEM). High-resolution transmission electron microscopy (HRTEM) confirmed not only the presence of well-dispersed CNTs but also needlelike shape $\mathrm{Al}_{4} \mathrm{C}_{3}$ crystals in the $\mathrm{Al}$ matrix. They also evaluated the mechanical behavior of the composites by Vickers micro-hardness measurements to indicate a significant improvement in hardness as a function of CNT content. This improvement was mainly associated to a homogeneous dispersion of CNTs in the Al matrix. Liu et al. [95] linked the formation of $\mathrm{Al}_{4} \mathrm{C}_{3}$ to the interaction between the outer shells of $\mathrm{CNT}$ s and the $\mathrm{Al}$ matrix during MA process in which recrystallization took place after the sintering process. Bakshi and Agarwal [97] stated that the strengthening efficiency of CNTs can be divided into three regimes of CNT content which showed decreasing extent of strengthening effect with increasing CNT content. They concluded that strengthening is highly dependent on CNT dispersion in powder mixture and that a higher degree of deformation during processing leads to increased strengthening. Hao et al. [29] proved that $\mathrm{CNT} /$ matrix chemical interaction is essential for strengthening, since toughness decreases for high CNT content ( $>5$ wt. \%) with poor dispersion.

\subsection{Summary}

The literature survey covered throughout this section, mainly helped understand the deformation behavior of $\mathrm{Mg}$ and $\mathrm{Al}$ alloys with and without respective $\mathrm{RE}$ and $\mathrm{CNT}$ additions. Deformation 
twinning, which could give rise to a radical reorientation of the grains, could lead to a considerable texture modification. It is therefore crucial to understand how twinning can contribute to the overall deformation behavior of the material. In addition, double twinning represents a particularly influential phenomenon in the deformation, fatigue and fracture of $\mathrm{Mg}$ alloys. It was observed throughout the available literature that the addition of RE elements was mainly to reduce the unfavorable mechanical anisotropy and tension-compression yield asymmetry of Mg alloys. Microstructures, crystallographic textures, and tensile and compressive properties in relation to the addition of RE elements have been previously reported, however, no fatigue data on the low-RE and low-cost ZEK100 Mg alloy under strain-controlled tests are available. It is unclear if cyclic hardening or softening would occur, to what extent the tensioncompression yield asymmetry would remain, whether pseudo-elastic behavior would still be present, and how it can be quantified. Finally, the extent of improvement that could be achieved in $\mathrm{Al}$ base alloys by introducing CNT reinforcement needs further explications in terms of different strengthening mechanisms involved, hot workability and high-temperature performance. 


\section{CHAPTER 3}

\section{EXPERIMENTAL PROCEDURE}

\subsection{Test Materials}

Investigations on the RE-free Mg alloy was $\mathrm{AZ} 31 \mathrm{Mg}$ alloy in the form of extruded plate, with a chemical composition shown in Table 3.1. The AZ31 Mg alloy was extruded in the temperature range of $360-382^{\circ} \mathrm{C}$ with an extrusion exit speed of $50.8 \mathrm{~mm} / \mathrm{s}$ and an applied extrusion ratio of 6. AZ31 was considered in the present study as "the model" RE-free Mg alloy to help understand the impact of RE additions. It was considered a long with other comparable materials in terms of chemical composition and fabrication process.

Table 3.1 Chemical composition of AZ31Mg alloy (wt.\%).

\begin{tabular}{llllllll}
\hline Base material & Al & Mn & Zn & Fe & Ni & Cu & Mg \\
\hline AZ31 & 3.1 & 0.54 & 1.05 & 0.0035 & 0.00007 & 0.0008 & Bal. \\
\hline
\end{tabular}

ZEK100 Mg alloy, with a composition listed in Table 3.2, was the subject of the studies about the low RE-containing $\mathrm{Mg}$ alloy in the form of rolled sheets under the annealing O condition which is the standard designation for the high ductility temper achieved by annealing the material at $500^{\circ} \mathrm{C}$ for $15 \mathrm{~min}$ in an electrical oven [98]. Fabrication of this material involved initial workpieces of $28.5 \mathrm{~mm}$. During rolling, the temperature of workpiece was $270^{\circ} \mathrm{C}$ and that of the rollers was $400^{\circ} \mathrm{C}$, with a rolling speed of $5 \mathrm{~m} / \mathrm{min}$, and no addition of lubricant. The thickness of the workpiece reduced from 28.5 to $3 \mathrm{~mm}$ in 5 passes. 
Table 3.2 Chemical composition of ZEK100 Mg alloy (wt.\%).

\begin{tabular}{lccccc}
\hline Base material & Zn & Zr & Nd & Mn & Mg \\
\hline ZEK100 & 1.3 & 0.25 & 0.2 & 0.01 & Bal. \\
\hline
\end{tabular}

AMCs investigations were conducted using a 2024Al base alloy (Table 3.3) and a $2.0 \mathrm{wt} . \% \mathrm{CNT}$ 2024Al composite. The composite was obtained after mixing 2.0 wt.\% CNTs with $2024 \mathrm{Al}$ alloy powders presented in Table 3.3. The average diameter of the $2024 \mathrm{Al}$ powders was $\sim 10 \mu \mathrm{m}$. The as-received CNTs (95-98\% purity) were synthesized using chemical vapor deposition (CVD). CNTs had entangled morphologies with a length of $>5 \mu \mathrm{m}$ and an outer diameter of 10 20 nm. The as-mixed powders of 2024Al and $2.0 \mathrm{wt} . \% \mathrm{CNTs}$ were dispersed by ball milling for 6 hours at a rotational rate of 400 RPM and a ball powder ratio of $15: 1$, using a stir milling machine with hardened steel balls. No extra pre-treatment was conducted on the CNTs before milling, however 2.0 wt.\% stearic acid was added to prevent serious cold-welding. The as-milled powders were cold-compacted in a cylinder die, degassed, then hot pressed at $560^{\circ} \mathrm{C}$ for $1 \mathrm{~h}$ into cylindrical billets. The as-pressed billets were hot extruded by our collaborators at $450^{\circ} \mathrm{C}$ with a ratio of $25: 1$. The 2.0 wt.\% CNT/2024Al composite was finally solid-solution-treated at $495^{\circ} \mathrm{C}$ for two hours, quenched in water at RT and then naturally aged (i.e., T4 condition).

Table 3.3 Chemical composition of 2024Al alloy (wt.\%).

\begin{tabular}{lcccc}
\hline Base material & Cu & Mg & Mn & Al \\
\hline 2024Al & 4.5 & 1.5 & 0.6 & Bal. \\
\hline
\end{tabular}




\subsection{Sample preparation and testing}

\subsubsection{Metallography}

Metallographic samples (approx. $5 \mathrm{~mm} \times 5 \mathrm{~mm}$ in the cross section) for the purpose of initial microstructural characterization were cut from the previously mentioned materials. The samples were cold mounted using LECO 7007 resin powder and liquid (mixing ratio: 2 parts of resin added to 1 part of catalyst). Hot mounting was avoided to prevent any possible microstructural change due to the effect of temperature during sample preparation. The mounted samples were manually ground with $\mathrm{SiC}$ sand papers with a grit number of 320,600 , and 1200 , respectively. Water was used as lubricant in each grinding step. Polishing was carried out with $6 \mu \mathrm{m}, 3 \mu \mathrm{m}$, and $1 \mu \mathrm{m}$ diamond paste using diamond extender (a mixture of rust inhibiting solution with distilled water $-10 \%$ solution by volume) as lubricant. Cleaning of the mount, after polishing, involved dipping and spraying with ethanol, ultrasonically cleaning in ethanol, followed by drying with compressed air. After the final polishing with colloidal silica, the polished samples were etched. Mg alloys (AZ31 and ZEK100) were etched with acetic picral (10 ml acetic acid, $4.2 \mathrm{~g}$ picric acid, $10 \mathrm{ml}$ distilled water, and $70 \mathrm{ml}$ ethanol). $\mathrm{Al}$ alloy and composite (2024Al and 2.0 wt.\% CNT 2024Al, respectively) were etched using Keller's etchant (i.e., $190 \mathrm{ml} \mathrm{H}_{2} \mathrm{O}, 5 \mathrm{ml}$ $\mathrm{HNO}_{3}(65 \%), 3 \mathrm{ml} \mathrm{HCl}(32 \%)$ and $\left.2 \mathrm{ml} \mathrm{HF}(40 \%)\right)$. No etching was done for the scanning electron microscope (SEM) observations, where the polished surfaces were examined directly. As for the EBSD examinations, sample surface was first mechanically polished, then electropolished in an electrolyte of $10 \mathrm{ml}$ nitric acid and $40 \mathrm{ml}$ ethanol for about $35 \mathrm{~s}$ and $20 \mathrm{~V}$ at RT for the $\mathrm{Al}$ alloy and composite, and $30 \mathrm{~s}$ and $20 \mathrm{~V}$ at RT for the two Mg alloys. 


\subsubsection{Quantitative image analysis}

Microstructural examinations were performed using the following techniques:

- An optical microscope (OM) equipped with Clemex quantitative image analysis software.

- A scanning electron microscope (SEM) JSM-6380LV equipped with Oxford energy dispersive X-ray spectroscopy (EDS) system and three-dimensional surface/fractographic analysis capacity.

- An electron backscatter diffraction (EBSD) analyses by means of Oxford integrated AZtecHKL advanced EBSD system with NordlysMax ${ }^{2}$ and AZtecSynergy along with a large area analytical silicon drift detector. EBSD analyses were carried out at a step size of $0.1 \mu \mathrm{m}$.

- A transmission electron microscope (TEM) and high resolution transmission electron microscope (HRTEM), performed in Shenyang National Laboratory for Materials Science, China, using TEM, Tecnai G2 20. TEM samples were machined parallel to ED.

\subsubsection{Phase identification by $\mathrm{X}$-ray diffraction}

Verification of the phases formed in the studied materials was done via X-ray diffraction (XRD) analysis. For this, a multi-functional PANalytical X'Pert PRO X-ray diffractometer was used to identify the formation of phases in the samples. XRD was performed using $\mathrm{Cu} K_{\alpha}$ radiation

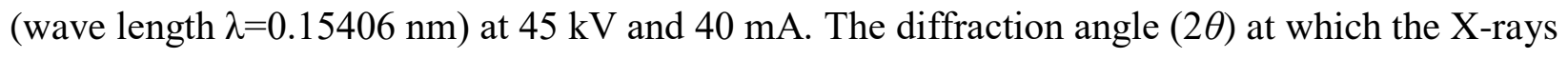
hit the sample varied from $40^{\circ}$ to $100^{\circ}$ with a step size of $0.04^{\circ}$ and $2 \mathrm{~s}$ in each step. 


\subsubsection{Texture measurements by $\mathrm{X}$-ray diffraction}

Texture measurements were performed on the as-received samples. The polishing procedure was the same as the one employed for microstructural observations and image analysis but without etching or electro-polishing. The texture was determined by measuring incomplete pole figures between $\Psi=0$ to $75^{\circ}$ in the back reflection mode using a PANalytical X'Pert PRO X-ray diffractometer with $\mathrm{Cu} K_{\alpha}$ radiation at $45 \mathrm{kV}$ and $40 \mathrm{~mA}$. The texture measurements were designed to determine the intensity variation of a certain diffraction peak, indexed $\boldsymbol{h}=(h k l)$, as a function of the measurement direction $(y)$ relative to the sample-reference frame. After corrections and normalizations, the probability maps, $P(\boldsymbol{h}, \boldsymbol{y})$, or pole figures were constructed to describe the distribution of different crystal directions in the sample space using the MTEX software or the Oxford AztecHKL software. Orientation distribution function (ODF) was also calculated from the measured data, where Bunge notations of the Euler angles $\left(\varphi_{1} \Phi \varphi_{2}\right)$ were implemented. Defocusing due to the rotation of XRD sample holder was corrected using experimentally determined data from Mg powders.

\subsection{Mechanical testing}

\subsubsection{Compression tests}

Two different categories of compression tests were performed in the present dissertation, which varied with the subject of the investigation. The two tests will be introduced separately below. 


\subsubsection{Stepwise compression tests}

These tests were performed on the AZ31 Mg alloy for the samples displayed in Fig.3.1.

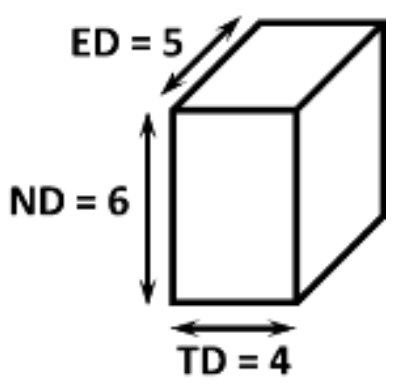

Fig.3.1. Illustration of the specimens for compression testing of AZ31 Mg alloy (unit in mm).

Sample configuration presented in Fig.3.1 allows loading along the three different directions with the same sample geometry. It also permits an easy preparation of a flat polished surface to quantify the deformation characteristics after each compression step. Tests were conducted at RT using a computerized Instron machine and the compressive strain amount was gradually increased. The strain was obtained based on crosshead displacement in the test apparatus, which included the deformation of both test sample and machine system (i.e., load train and machine frame). In evaluating the stress-strain curves, to obtain the actual deformation amount of a test sample, the deformation coming from the machine system was eliminated using a calibration curve which was obtained directly through the upper compression plate against the lower compression plate without any sample. Then all of the reported values in this study are actual strain values with the machine deformation amount excluded using the above-mentioned plateto-plate calibration curve. 


\subsubsection{Continuous compression tests}

Cylindrical specimens (Fig.3.2) were machined for the 2024Al base alloy and the 2.0 wt.\% CNT 2024Al composite in concordance with ASTM E9-09 standard, with the compression axis parallel to ED. Compression tests were carried out until failure at RT, and in the range of 200$400^{\circ} \mathrm{C}$, at strain rates of $0.001,0.01$, and $0.1 \mathrm{~s}^{-1}$, using a computerized United testing machine equipped with an environmental chamber having a temperature accuracy of $\pm 5^{\circ} \mathrm{C}$. A calibration curve at each temperature was obtained to get rid of the machine deformation in evaluating the stress-strain curves. To verify the reproducibility of the results, two tests were performed in each deformation condition (i.e., each combination of strain rate and deformation temperature).

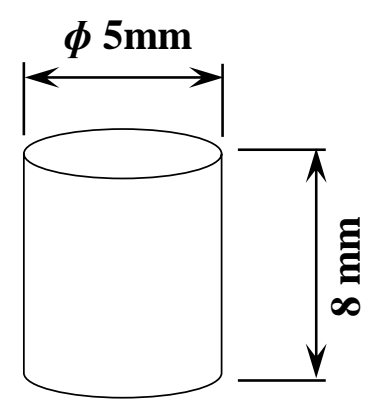

Fig.3.2. Illustration of the specimen for compression testing on $2024 \mathrm{Al}$ and the $2.0 \mathrm{wt} . \%$ CNT/2024 Al composite.

\subsubsection{Tensile tests}

Sub-sized tensile samples in accordance with ASTM E8 standard were machined with the loading axis parallel to ED. The sample geometry and dimensions are shown in Fig.3.3. Tensile 
tests were performed by means of a computerized UNITED tensile testing machine with a sample gauge length of $25 \mathrm{~mm}$ (or a parallel length of $32 \mathrm{~mm}$ ) at a strain rate of $1 \times 10^{-2} \mathrm{~s}^{-1}$.

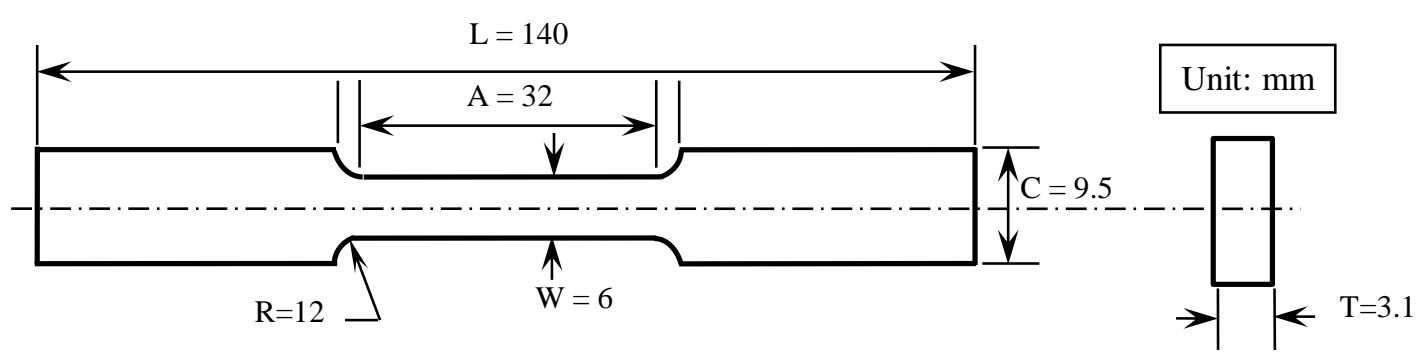

Fig.3.3. Illustration of the dimensions of the sub-sized tensile test specimens.

\subsubsection{Fatigue tests}

Strain-controlled, pull-push type fatigue tests were conducted using a computerized servohydraulic Instron 8801 fatigue testing system via a Fast Track Low Cycle Fatigue (LCF-3) program at a constant strain rate of $1 \times 10^{-2} \mathrm{~s}^{-1}$ and $\mathrm{RT}\left(\sim 25^{\circ} \mathrm{C}\right)$. Fatigue specimens are displayed in Fig.3.4. The gauge section of fatigue samples was ground progressively along the loading direction (LD) with emery papers up to a grit number of 600 to remove the machining marks and to achieve a consistent surface. Tests were carried out in a strain control mode according to ASTM E606. Triangular strain waveform was applied during the tests. LCF tests were performed at zero mean strain $\left(R_{\varepsilon}=-1\right.$, completely reversed strain cycle), at total strain amplitudes of $0.2 \%$, $0.4 \%, 0.6 \%, 0.8 \%, 1.0 \%$, and $1.2 \%$, and at least two samples were tested at each level of the strain amplitudes. The calibration of both load and strain channels was performed for each individual sample prior to testing. The strain-controlled tests at lower strain amplitude levels were continued up to 10,000 cycles, then the tests were changed to load control at a frequency of $50 \mathrm{~Hz}$ using sine waveform. The fracture surfaces of fatigued specimens were examined via 
SEM to identify fatigue crack initiation sites and propagation characteristics. The residual twins in the region near the fracture surface were observed as well.

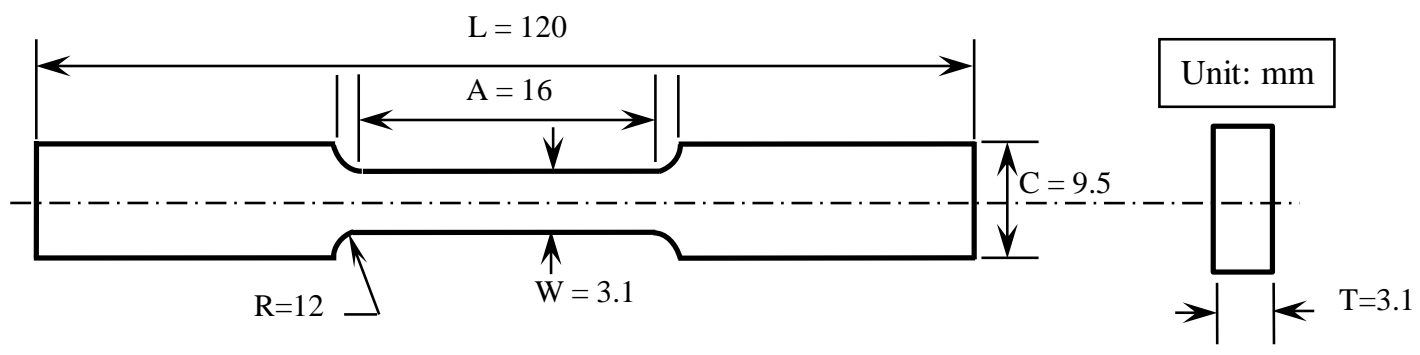

Fig.3.4. Illustration of the dimensions of the sub-sized fatigue test specimens.

To study the effect of strain ratio on the LCF behavior of the ZEK100 Mg alloy, five different strain ratios, i.e., $R_{\varepsilon}=0.5,0,-1,-3$, and $-\infty$, were used at a given total strain amplitude of $0.8 \%$ and at least two samples were tested at each level of the strain ratio. Test parameters used in LCF tests under different strain ratios at constant amplitude of $0.8 \%$ and a strain rate $1 \times 10^{-2} \mathrm{~s}^{-1}$ are listed in Table 3.4 .

Table 3.4 Test parameters under different strain ratios at strain amplitude of $0.8 \%$ and a strain rate of $1 \times 10^{-2} \mathrm{~s}^{-1}$.

\begin{tabular}{cccc}
\hline $\begin{array}{c}\text { Strain ratio } \\
\left(\boldsymbol{R}_{\varepsilon}\right)\end{array}$ & $\begin{array}{c}\text { Mean strain } \\
\left(\boldsymbol{\varepsilon}_{\text {mean }}\right), \boldsymbol{\%}\end{array}$ & $\begin{array}{c}\text { Maximum strain } \\
\left(\boldsymbol{\varepsilon}_{\text {max }}\right), \%\end{array}$ & $\begin{array}{c}\text { Minimum strain } \\
\left(\boldsymbol{\varepsilon}_{\text {min }}\right), \boldsymbol{\%}\end{array}$ \\
\hline 0.5 & 2.4 & 3.2 & 1.6 \\
0 & 0.8 & 1.6 & 0 \\
-1 & 0 & 0.8 & -0.8 \\
-3 & -0.4 & 0.4 & -1.2 \\
$-\infty$ & -0.8 & 0 & -1.6 \\
\hline
\end{tabular}


The varying strain ratio tests were performed in the same manner at a strain rate of $1 \times 10^{-2} \mathrm{~s}^{-1}$ and RT with triangular waveform loading. After fatigue testing, SEM was used to examine the fatigue crack initiation sites and identify the mechanism of fatigue crack propagation under the above applied conditions. 


\section{CHAPTER 4}

\section{DEFORMATION BEHAVIOR OF A RE-FREE AZ31 Mg ALLOY DURING STEPWISE COMPRESSION}

\subsection{Introduction}

The objective of this investigation is to identify the twinning characteristics in a RE-free

extruded AZ31 Mg alloy under favorable conditions of profuse $\{10 \overline{1} 2\}$ extension twinning using in-situ optical microscopy, electron backscatter diffraction, and X-ray diffraction analysis. Various double twinning structures acknowledged theoretically or through atomistic simulations were confirmed experimentally.

\subsection{Microstructural characterization upon compression}

Fig.4.1 shows the true stress-true strain curve of the extruded AZ31 Mg alloy compressed in the $\mathrm{ED}$, along with distinct microstructures at different true strain amounts up to $\sim 10.8 \%$. Several typical areas on the sample surface were observed after each compression, aiming to capture distinctive, typical and special twinning features from the polished sample surface. It is seen from Fig.4.1 that prior to deformation, the alloy exhibited a twin-free microstructure, consisting of a mix of large and small grains (Fig.4.1(a)). Some lenticular traces of extension twinning appeared at $\varepsilon=2.5 \%$ (Fig.4.1(b)), which were basically parallel to each other in a given grain. As the true strain amount increased to $\sim 4.3 \%$ (Fig.4.1(c)) and $\sim 6.0 \%$ (Fig.4.1(d)), twins were broadened and their morphology became more complicated (i.e., more misalignment was 
observed within a single grain). A few distinct features started to emerge; hence the highlighted locations (i.e., $\mathrm{L}_{1}$ to $\mathrm{L}_{7}$ dashed boxes in Fig.4.1) will be studied in more details below. It should be noted that, to avoid the loss of generality, locations $\mathrm{L}_{1}$ to $\mathrm{L}_{7}$ were indicated on the micrographs of Fig.4.1 after carefully examining the whole microstructure so as to identify the most typical and prevailing deformation features.

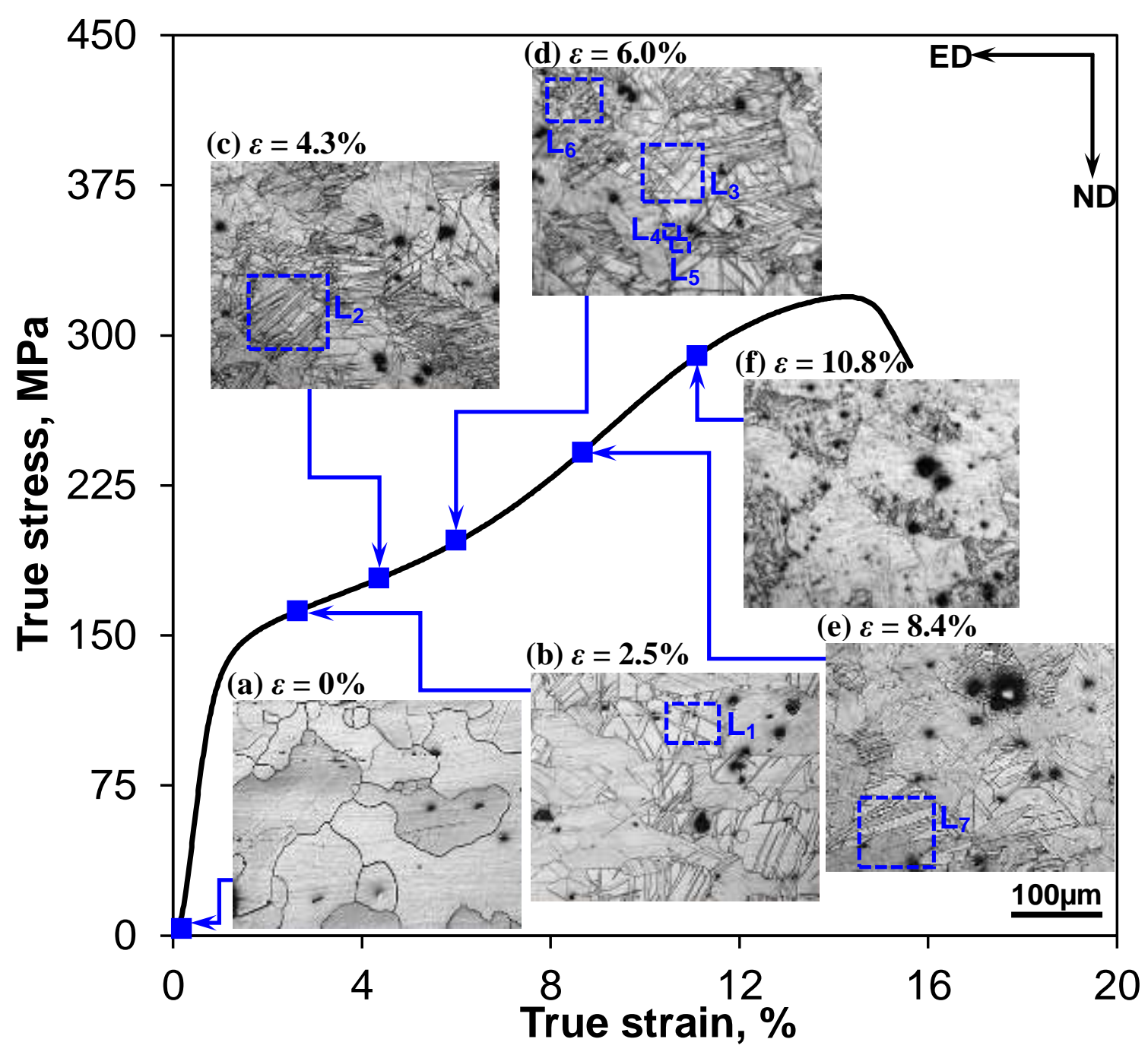

Fig.4.1 Typical compressive true stress-true strain curve along with microstructural changes at varying true strains of (a) $0 \%$, (b) $2.5 \%$, (c) $4.3 \%$, (d) $6.0 \%$, (e) $8.4 \%$, and (f) $10.8 \%$. 
At a compressive true strain of $\sim 8.4 \%$ (Fig.4.1(e)), fewer twins were seemingly seen compared with Fig.4.1(c) and (d), indicating that twin growth occurred via the coalescence of twins or the vanishing of twin boundaries. Extension twins were observed to merge together, sweeping through the entire area of a grain as highlighted in $\mathrm{L}_{7}$ in Fig.4.1(e). In addition, some narrow banded twins started to appear at $\varepsilon \sim 6.0 \%$ leading to the formation of double twins $\left(\mathrm{L}_{4}\right.$ and $\mathrm{L}_{5}$ in Fig.4.1(d)). Twin-twin interactive structures were observed at various true strains throughout the micrographs (e.g., $\mathrm{L}_{1}$ in Fig.4.1(b) at $\varepsilon \sim 2.5 \%$, and $\mathrm{L}_{6}$ in Fig.4.1(d) at $\varepsilon \sim 6.0 \%$ ) and will be explained further below. Most of the matrix was swept at last at a strain of $10.8 \%$ and twins eventually encompassed the entire grain (Fig.4.1(f)).

\subsection{Evolution of crystallographic texture}

$\{0001\}$ pole figures of the extruded AZ31 Mg alloy at different strain amounts are displayed in Fig.4.2. At $\varepsilon=0 \%$, almost all $c$-axes of the grains were nearly perpendicular to the ED (i.e., most basal planes of the grains were parallel to the ED). This orientation is favorable for extension twinning during compression along ED. The initial basal texture was fairly strong with a maximum intensity of $~ 7.7 \mathrm{MRD}$ (multiples of random distribution) and significant changes were detected during compression. At a strain of 2.5\% (Fig.4.2(b)), basal $\{0001\}$ poles began a split towards ED, however the center maintained a higher intensity of $\sim 9.4$ MRD. With increasing strain levels from $\varepsilon=4.3 \%$ (Fig.2(c)) to $8.4 \%$ (Fig.4.2(e)), more rotations of $c$-axes towards ED, opposing the compression direction, were observed where the maximum intensity increased from $\sim 5.1$ to $~ 9.1$ MRD. 


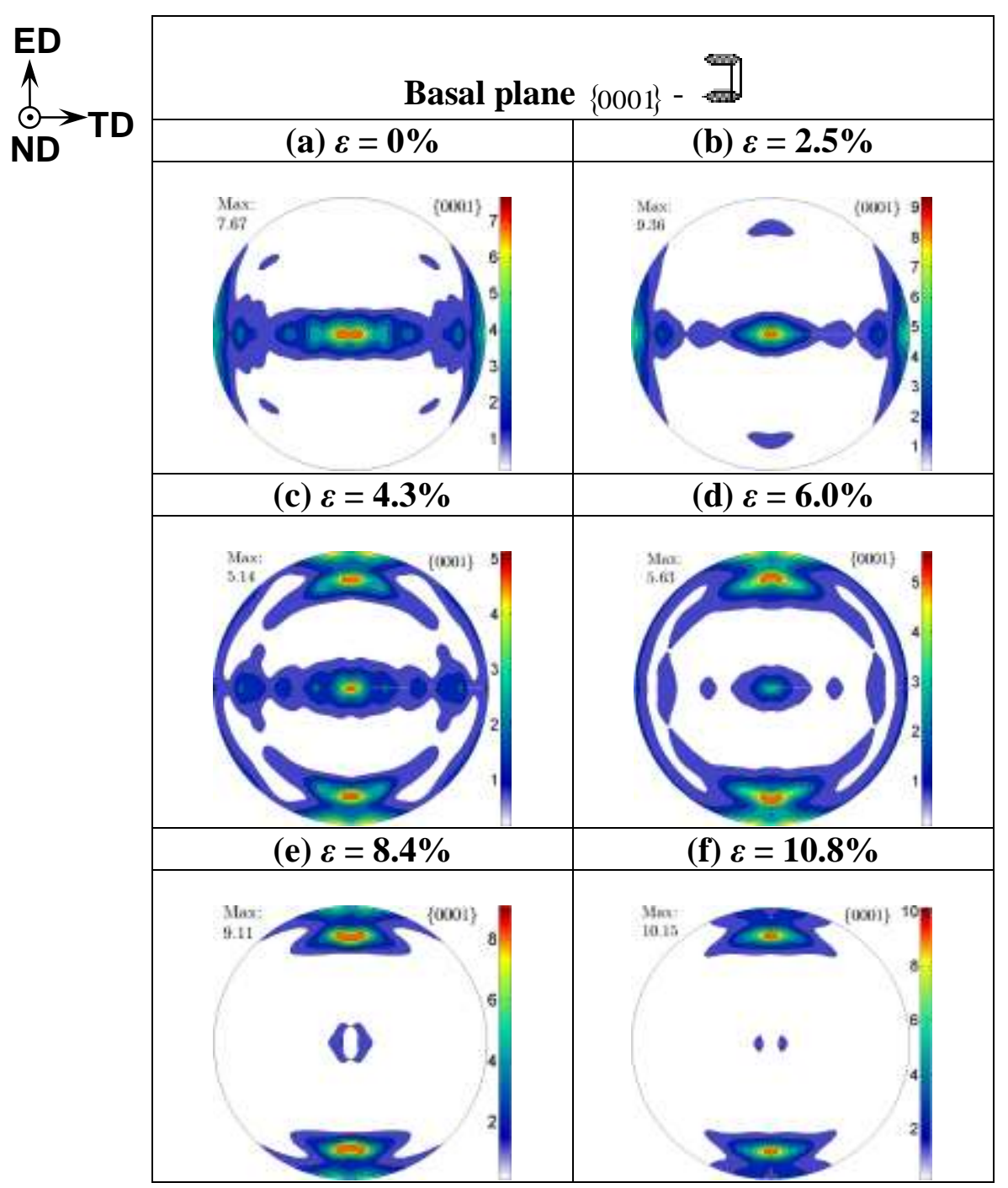

Fig.4.2 $\{0001\}$ pole figures obtained from (a) the undeformed sample and the compressed samples of the extruded AZ31 Mg alloy at true strain amounts of (b) $2.5 \%$, (c) $4.3 \%$, (d) $6.0 \%$, (e) $8.4 \%$ and (f) $10.8 \%$. Color code adjusted automatically.

The $c$-axes of further more grains were directed to ED after $10.8 \%$ strain (Fig.4.2(f)) with a further higher maximum intensity of $\sim 10.2 \mathrm{MRD}$, where almost no grains with the $c$-axes towards the center were left. Specific texture components developed during compression could be analyzed through the ODF. Bunge notations of Euler angles $\left(\varphi_{1} \Phi \varphi_{2}\right)$ were used for that purpose in Fig.4.3(a). A description of the orientation involving sequential rotations of the 
crystal coordinate system through three angles with respect to the specimen coordinate system was provided in Fig.4.3(b). Using such a crystal coordinate system, orientations could be specified from the Euler angles and the relationship between Euler angles $\left(\varphi_{1} \Phi \varphi_{2}\right)$ and texture components $\{h k i l\}\langle u v t w\rangle$ in the Bunge system could be expressed by the following equations [99],

$$
\begin{gathered}
{\left[\begin{array}{l}
h \\
k \\
i \\
l
\end{array}\right]=\left[\begin{array}{ccc}
\frac{\sqrt{3}}{2} & -\frac{1}{2} & 0 \\
0 & 1 & 0 \\
-\frac{\sqrt{3}}{2} & -\frac{1}{2} & 0 \\
0 & 0 & \frac{c}{a}
\end{array}\right] \times\left[\begin{array}{c}
\sin \varphi_{2} \sin \phi \\
\cos \varphi_{2} \sin \phi \\
\cos \phi
\end{array}\right],} \\
{\left[\begin{array}{l}
u \\
v \\
t \\
w
\end{array}\right]=\left[\begin{array}{ccc}
\frac{2}{3} & -\frac{1}{3} & 0 \\
0 & \frac{2}{3} & 0 \\
-\frac{2}{3} & -\frac{1}{3} & 0 \\
0 & 0 & \frac{c}{a}
\end{array}\right] \times\left[\begin{array}{c}
\cos \varphi_{1} \cos \varphi_{2}-\sin \varphi_{1} \sin \varphi_{2} \cos \phi \\
-\cos \varphi_{1} \sin \varphi_{2}-\sin \varphi_{1} \cos \varphi_{2} \cos \phi \\
\sin \varphi_{1} \sin \phi
\end{array}\right] .}
\end{gathered}
$$

ODFs help describe the frequency of occurrence of a particular orientation in the Euler space chosen in the case of Fig.4.3(a) to be limited by $0^{\circ} \leq \varphi_{1} \leq 90^{\circ}, 0^{\circ} \leq \Phi \leq 90^{\circ}$ and $0^{\circ} \leq \varphi_{2} \leq 60^{\circ}$ based on the hexagonal crystal symmetry. Main texture components (TCs) could be seen from Fig.4.3(a) at varying strain amounts, since the identification of the main TCs relies on the localization of the maximum intensities throughout all compression stages. For instance, if a component is initially identified as a maximum intensity (e.g., $\mathrm{TC}_{1}$ at $\varepsilon=0 \%$ and $\varphi_{2}=0^{\circ}$ with an intensity of $\sim 23.7 \mathrm{MRD}$ ), its intensity is determined and plotted throughout all compression stages even when it vanishes later. 


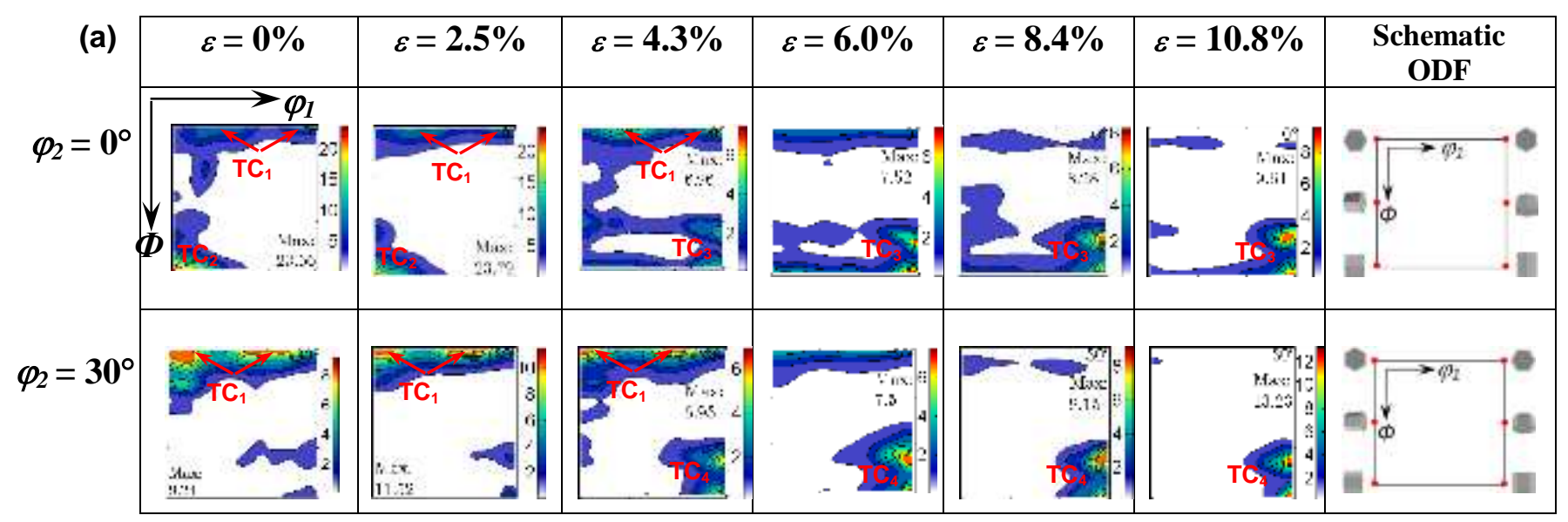

Fig.4.3(a)
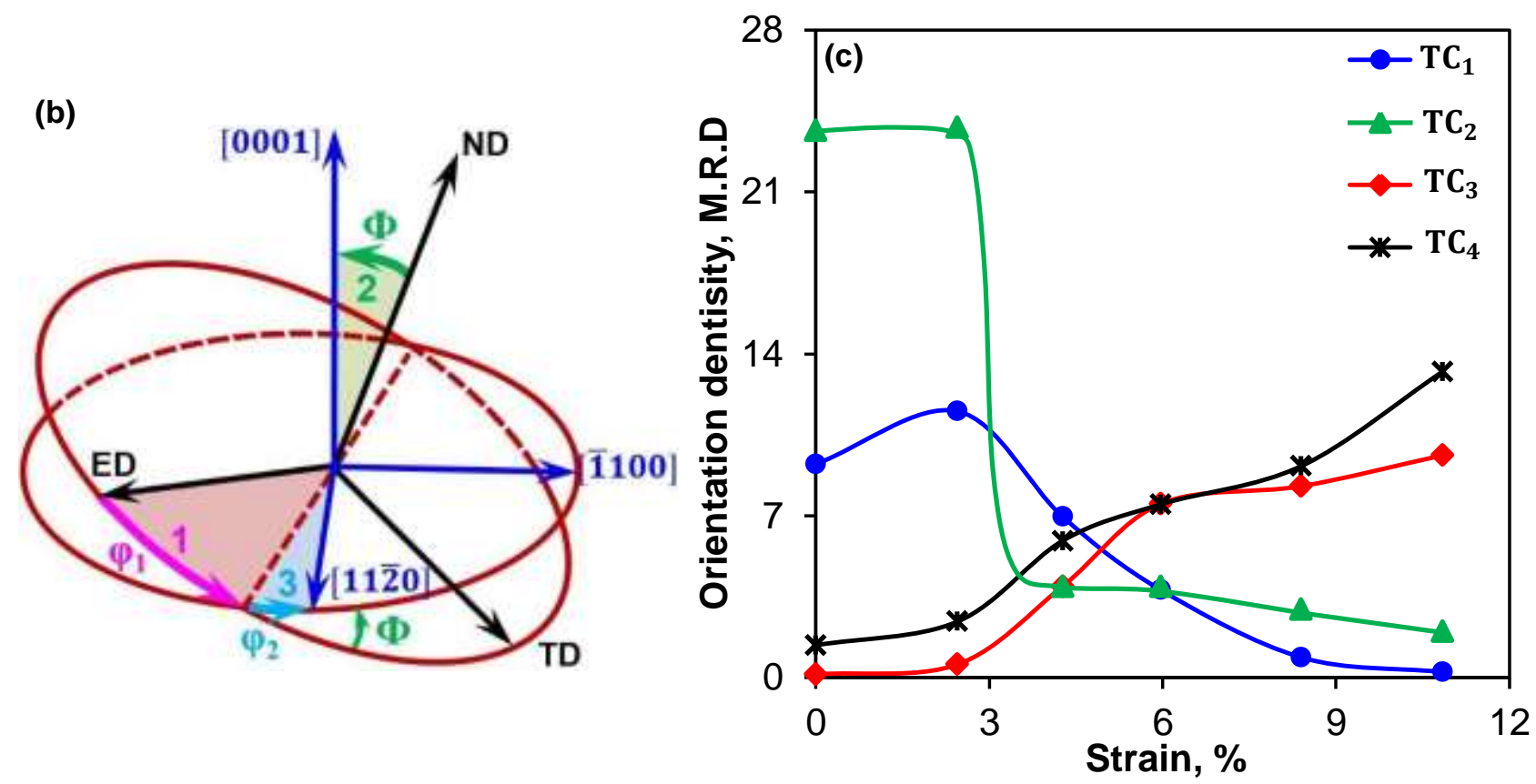

Fig.4.3(b, c)

Fig.4.3 (a) ODF sections at $\varphi_{2}=0^{\circ}$ and $\varphi_{2}=30^{\circ}$ of samples compressed at $0 \%, 2.5 \%, 4.3 \%, 6.0 \%$, $8.4 \%$ and $10.8 \%$, (b) definition of the crystal coordinate system based on the rotation of the specimen coordinate system through the Euler angles $\varphi_{1}, \Phi, \varphi_{2}$ in accordance with the Bunge definition in the order of $1,2,3$, and (c) evolution of main texture components with the strain.

Similarly, if a component is observed to be strengthened at later compression stages (e.g., $\mathrm{TC}_{3}$ and $\mathrm{TC}_{4}$ ) its evolution trend is monitored since earlier compression stages. After locating the 
desired TCs, associated Euler angles are identified from the ODFs. Based on the Euler angles and using the raw data generated during XRD texture measurements to construct the $\mathrm{ODF}_{\mathrm{S}}$, intensities associated with the identified angles could be determined and plotted in Fig.4.3(c). Table 4.1 lists the Euler angles of the identified four major texture components, where the planes and directions are indicated in the hcp unit cell. Note that the unit cell was positioned based on the crystal coordinate system presented in Fig.4.3(b). Euler angles resulting in the same TCs based on Equ.(4.1) and (4.2) have been grouped together in Table 4.1. Based on Fig.4.3(a), the undeformed sample had two main components $\mathrm{TC}_{1}\{0001\}\{2 \overline{1} \overline{1} 0\}$ at Euler angles of (30, 0, 0), (90, $0,0),(0,0,30),(60,0,30)$ and $\mathrm{TC}_{2}\{\overline{1} 2 \overline{1} 0\}\{10 \overline{1} 0\rangle$ at Euler angles of $(0,90,0) . \mathrm{TC}_{1}$ was reported by Hirsch and Al-Samman [13] to be among the most important texture components in extruded Mg alloys. Yi et al. [100] also identified an initial component similar to $\mathrm{TC}_{2}$ when studying the texture evolution in AZ31 during uniaxial loading. As the compressive strain continued to increase, $\mathrm{TC}_{1}$ and $\mathrm{TC}_{2}$ tended to disappear while two new major components, namely $\mathrm{TC}_{3}$ $\{12 \overline{1} 0\}\{0001\rangle$ at Euler angles of $(90,70,0)$ and $(90,90,0)$ and $\operatorname{TC}_{4}\{01 \overline{1} 0\}\langle 0001\rangle$ at Euler angles of $(90,70,30)$ and $(90,90,30)$ started to form. It is clear that at a strain of $10.8 \%, \mathrm{TC}_{1}$ and $\mathrm{TC}_{2}$ almost fully transformed into $\mathrm{TC}_{3}$ and $\mathrm{TC}_{4}$, as seen from Fig.4.3(a), reflecting the rotation of the $c$-axes towards the ED as previously observed in Fig.4.2. Fig.4.3(c) quantifies the change of their intensity with the compressive strain, where $\mathrm{TC}_{1}$ and $\mathrm{TC}_{2}$ initially experienced a slight increase at $\varepsilon=2.5 \%$ before fading away at higher strain amounts. This could be linked to the deviation of the maximum intensities towards the center combined with the slight ED-split that was previously observed in Fig.4.2 at the same strain amount. Starting from $\varepsilon=4.3 \%$, initial components weakened while $\mathrm{TC}_{3}$ and $\mathrm{TC}_{4}$ gradually intensified (Fig.4.3(c)). 
Table 4.1 Main texture components identified from Euler angles in accordance with the specified crystal coordinate system in Fig.4.3(b).

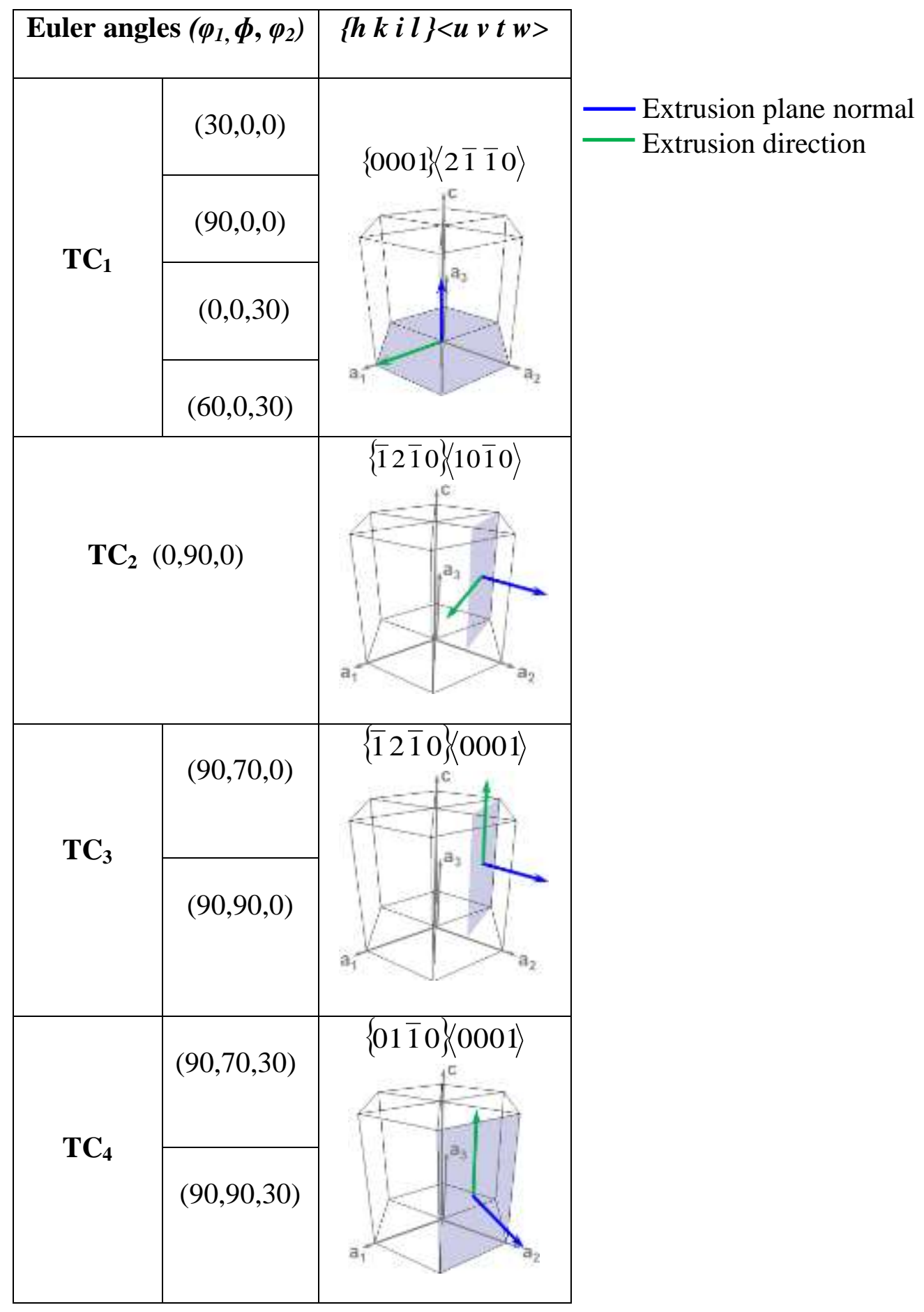




\subsection{EBSD analysis}

EBSD facilitated the study the samples compressed along ED at different strain amounts. Examinations of various twinning scenarios were focused in three typical regions encompassing a few relatively large grains that will be chosen to be studied separately. At each increment of true strain, the same grains were observed to depict the twinning behavior during stepwise deformation. The present EBSD studies used normal-projected orientation maps and a map color legend projecting the direction mutually normal to the ED and the observed surface.

\subsubsection{Twinning evolution in zone 1}

The orientation maps of the extruded AZ31 sample (zone 1) compressed at different strain amounts along with their corresponding inverse pole figures (IPFs) are shown in Fig.4.4. Similar to Fig.4.1, there was no twins present in the undeformed state (Fig.4.4(a)); with increasing strain $\left\{10 \overline{1}_{2}\right\}$ extension twins formed, grew and coalesced. Close observations indicated that most grains in zone 1 seemed to endure a single twin variant, i.e., most of the twins in a given grain were parallel to each other. The initial IPF orientation component of zone 1 confirmed the presence of mainly red (i.e., <0001>-oriented grains) prior to deformation, indicating the presence of basal textures as previously observed through XRD crystallographic texture analysis in Fig.2. With increasing strain, lenticular-shaped extension twins were observed to nucleate and grow, leading to the appearance of some new twin segments due to the further increase in the width of twin bands. When the true strain reached $8.6 \%$, the parent grains in zone 1 were almost totally swept by the $\{10 \overline{1} 2\}$ extension twinning and some smaller subgrains were observed. 

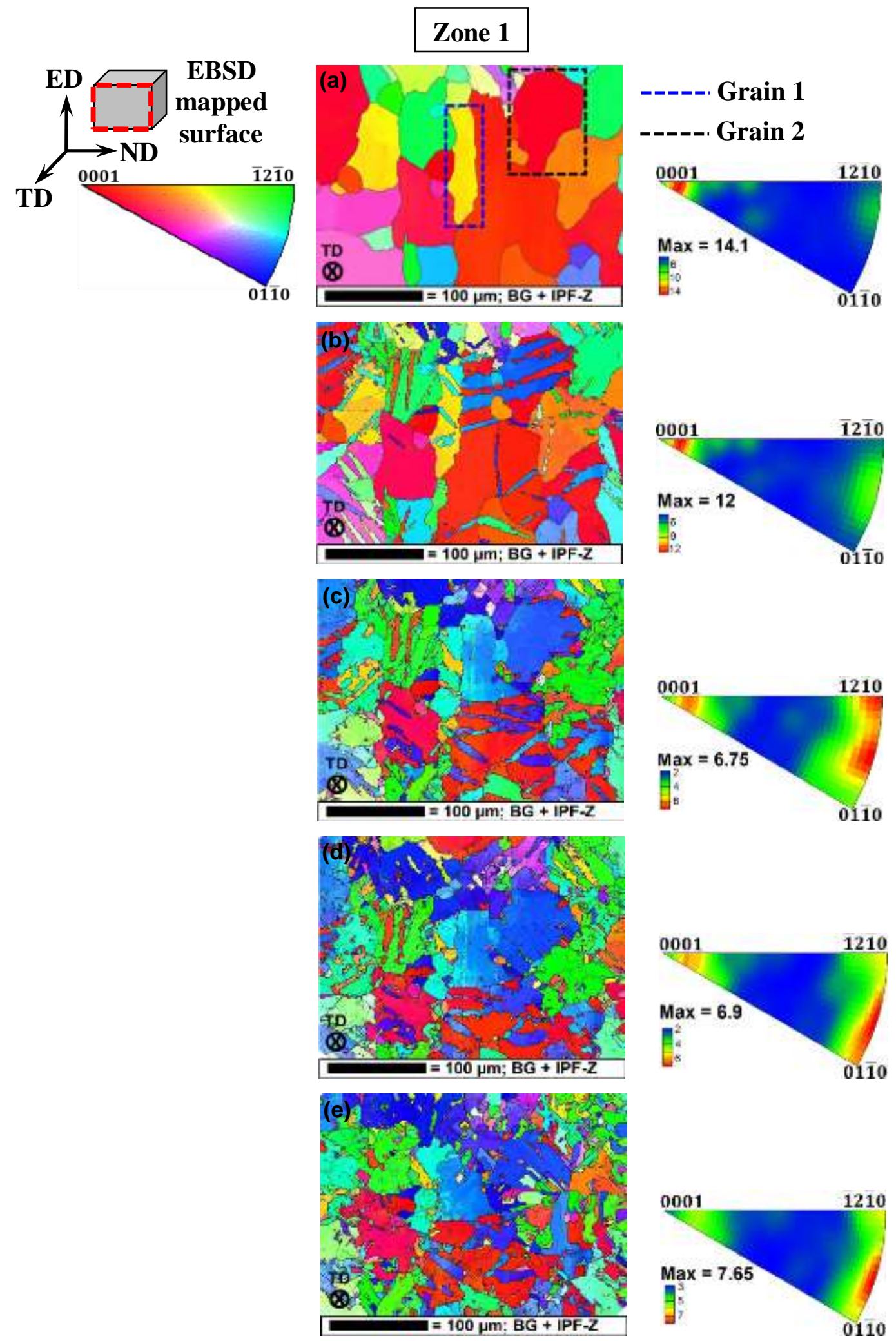

Fig.4.4 Normal-projected EBSD orientation maps and the corresponding inverse pole figures of zone 1 strained along ED at (a) $0 \%$, (b) $2.5 \%$, (c) $4.9 \%$, (d) $6.8 \%$ and (e) $8.6 \%$. 
IPF orientation components shown in Fig.4.4 along with the orientation maps allowed concluding that, throughout the compression steps, more $c$-axes rotated towards the compression direction ED. This was revealed by the change of the maximum intensity on the IPF orientation components from a majority of $<0001>$-oriented grains (at $\varepsilon=0 \%$ ) to a predominant direction between $\langle 01 \overline{1} 0\rangle$ and $\langle\overline{1} 2 \overline{1} 0\rangle($ at $\varepsilon=8.6 \%$ ), in agreement with the Fig.4.2.

\subsubsection{Twin propagation in zone 2 and zone 3}

Another interesting EBSD feature observed is shown in Fig.4.5, which was obtained through the EBSD forward scatter detector (FSD) images of zone 2, facilitating the examination of both the matrix/parents and deformation twins. As the strain amount increased from $0 \%$ to $6.8 \%$, micromechanics of extension twinning became more obvious through the motion of twin boundaries and the twinning-like lattice reorientation. Thus, FSD images went from nearly "flat surfaces" at $\varepsilon=0 \%$ (Fig.4.5(a)) to highly misaligned and uneven surfaces at $\varepsilon=6.8 \%$ (Fig.4.5(e)), where the imposed plastic strain on the parent grain was accommodated by the nucleation and glide of twinning dislocations at the twin boundaries. El Kadiri et al. [52] stated that "rough" twin boundaries were generated as the twins grew increasingly and slip in the parents was detected, causing the formation of local recessions and leading to the observed deformation of both parents and twins, as seen in Fig.4.5(e). In comparison with zone 1 (Fig.4.4), more twins intersecting each other in the same grain were observed in zone 2, where instances of twins branching to other twin systems occurred. In the previously observed zone 1 (Fig.4.4), at $\varepsilon$ $=6.8 \%$, twins were observed to be embedded in the original matrix by thickening or widening. In contrast, in zone 2 at the same strain (Fig.4.5(f)), a majority of slow-growing second-variant 
twin systems were observed, resulting in a more limited growth rate. Further details about the growth of $\{10 \overline{1} 2\}$ extension twins under single and double twin variants are discussed below.

\section{Zone 2}
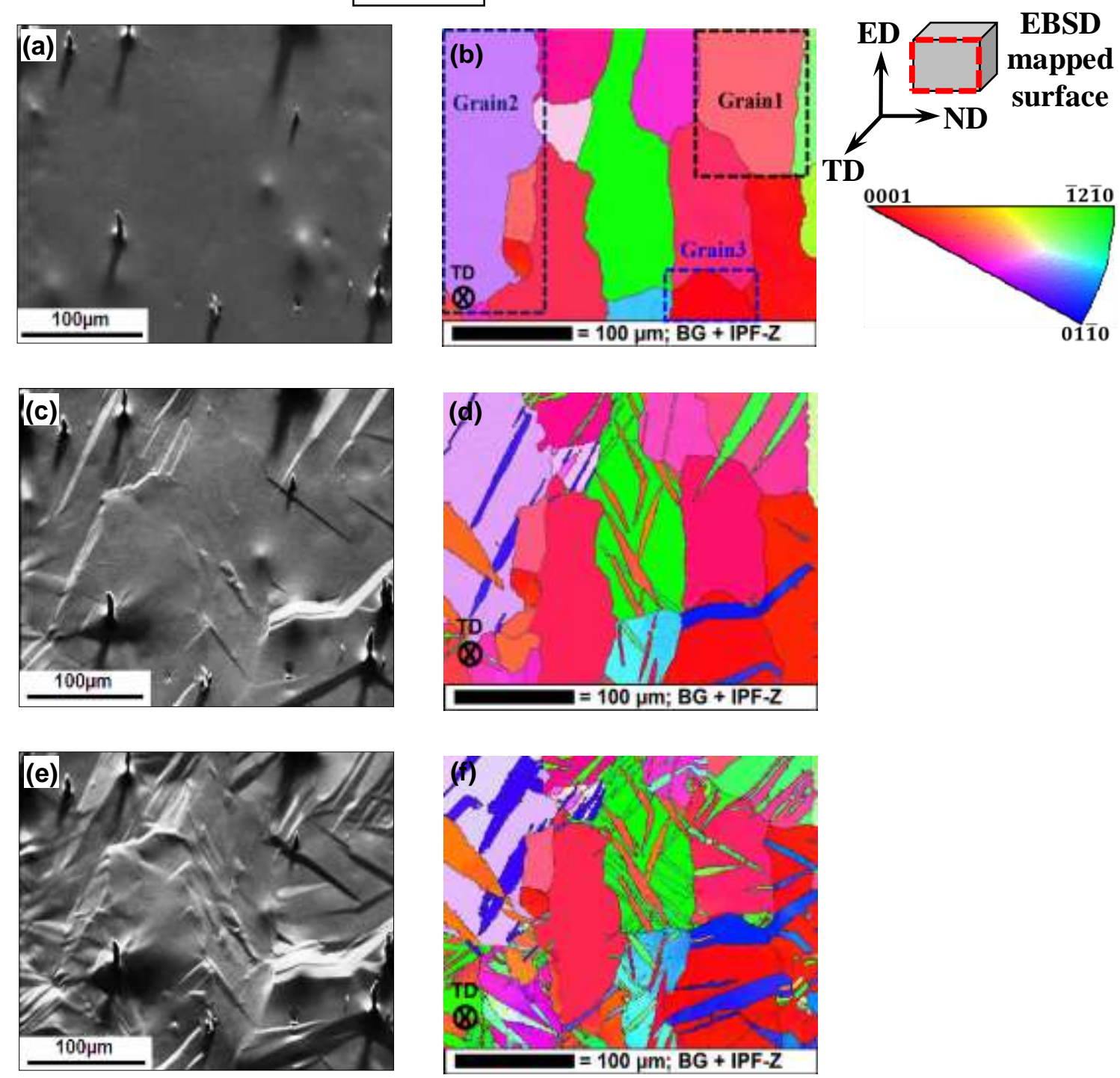

Fig.4.5 EBSD forward scatter detector (FSD) images and corresponding normal-projected orientation maps of zone 2 strained at (a,b) $0 \%$, (c, d) $4.9 \%$ and (e, f) $6.8 \%$, respectively.

A third region has been studied via EBSD analysis, namely zone 3 in Fig.4.6 with a smaller initial true strain of 1.2\% (Fig.4.6(b)), in comparison with zones 1 and 2 (Figs 4.4 and 4.5) which 
started with $2.5 \%$ compressive strain. Zone 3 was therefore used for the detection of finer twinning structures appearing at early stages and was scanned with a smaller EBSD step size. More distinctive features were observed throughout the compressive steps of $2.5 \%$ (Fig.4.6(c)), 5.5\% (Fig.4.6(d)), and 8\% (Fig.4.6(e)), and a few selected grains (indicated by the dashed boxes in Fig.4.6(a)) and specific positions (indicated in Fig.4.6(e)) are reported separately below.
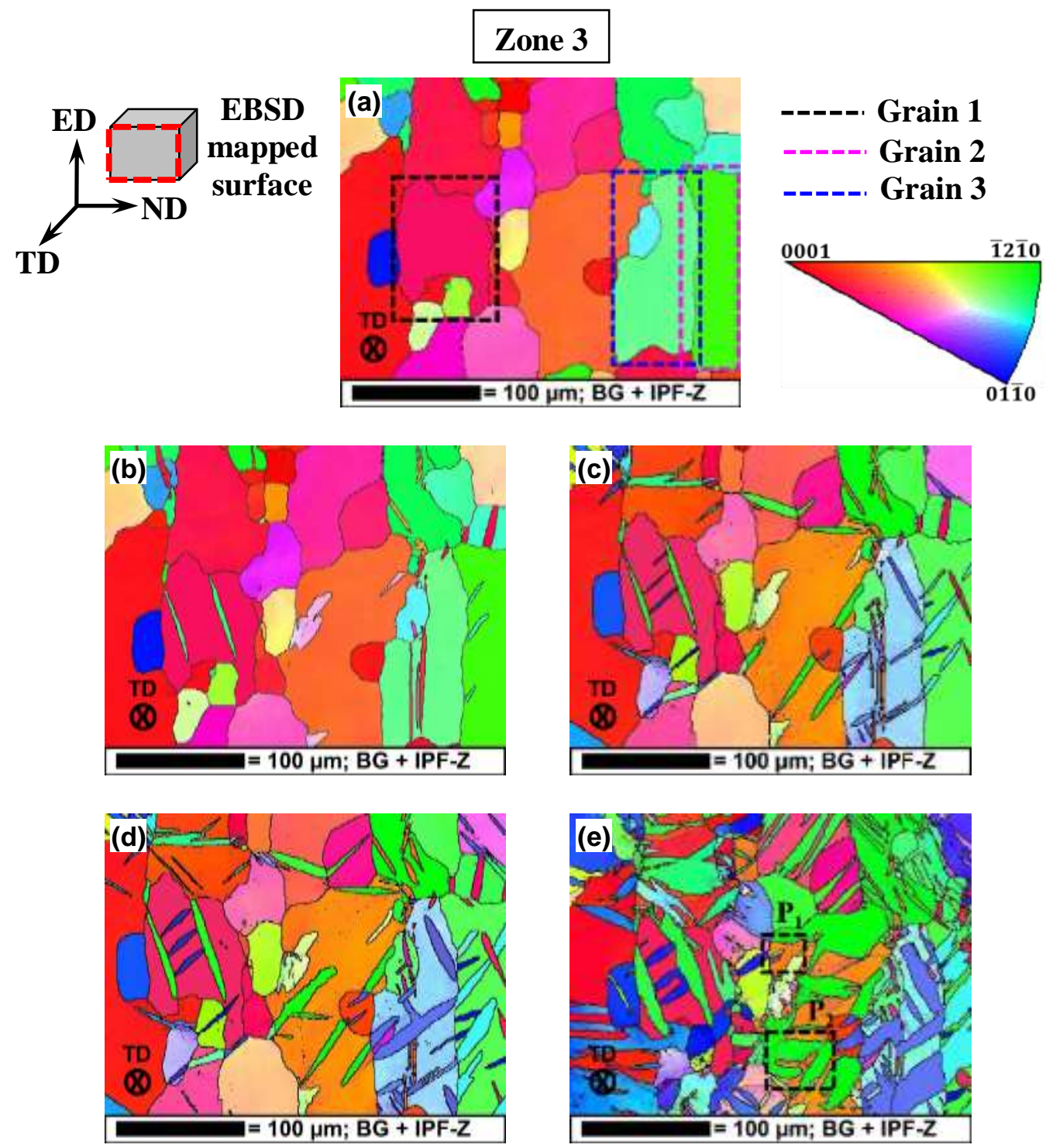

Fig.4.6 Normal-projected EBSD orientation maps of zone 3 strained along ED at (a) $0 \%$, (b) $1.2 \%$, (c) $2.5 \%$, (d) $5.5 \%$ and (e) $8 \%$. 


\subsection{Discussion}

\subsubsection{Effect of the grain shape on the growth of $\{10 \overline{1} 2\}$ twins}

It is known that the activation of the main twinning system $\{10 \overline{1} 2\}\{10 \overline{1} 1\rangle$ provokes a misorientation of $\sim 86.3^{\circ}$ between the twinned and untwinned lattice [100]. Zone 1-grain 1 in Fig.4.7, undergoing stepwise compressions up to a strain of $4.9 \%$ showed an initial misorientation profile crossing the originally untwinned grain $\mathrm{A}-\mathrm{B}$ at $\varepsilon=0 \%$, which displayed just a noisy background (Fig.4.7(b)), i.e., a low angle misorientations of $\sim 1^{\circ}$. A few lenticular twins appeared at $\varepsilon=2.5 \%$, with several peaks of $\sim 86^{\circ}$ seen in the misorientation profile, exactly corresponding to the number of twin boundaries intersected by line A - B (Fig.4.7(d)). At a strain of $4.9 \%$ (Fig.4.7(e)), it is of particular interest to observe that the position of misorientation peaks changed, indicating the movement of extension twin boundaries; the decrease in the number of misorientation peaks suggested the coalescence or merging of neighboring twins.

Twin growth in zone 1 - grain 2 (Fig.4.8(a)), which had a different grain shape from zone 1 grain 1 was further examined. The evolution with respect to the stepwise compression is shown in Fig.4.8(a-c), where the lattice orientation is represented by the schematic hcp unit cell; in agreement with the previously observed results via pole figures (Fig.4.2) and EBSD orientation maps (Fig.4.4), the $c$-axis of the grain was nearly perpendicular to the ED at $\varepsilon=0 \%$ (Fig.4.8(a)). Three major extension twins were observed to form at $\varepsilon=2.5 \%$, which were accommodated by a $\sim 86^{\circ}$ rotation of schematic hcp unit cells with respect to the untwinned matrix (Fig.4.8(b)). 

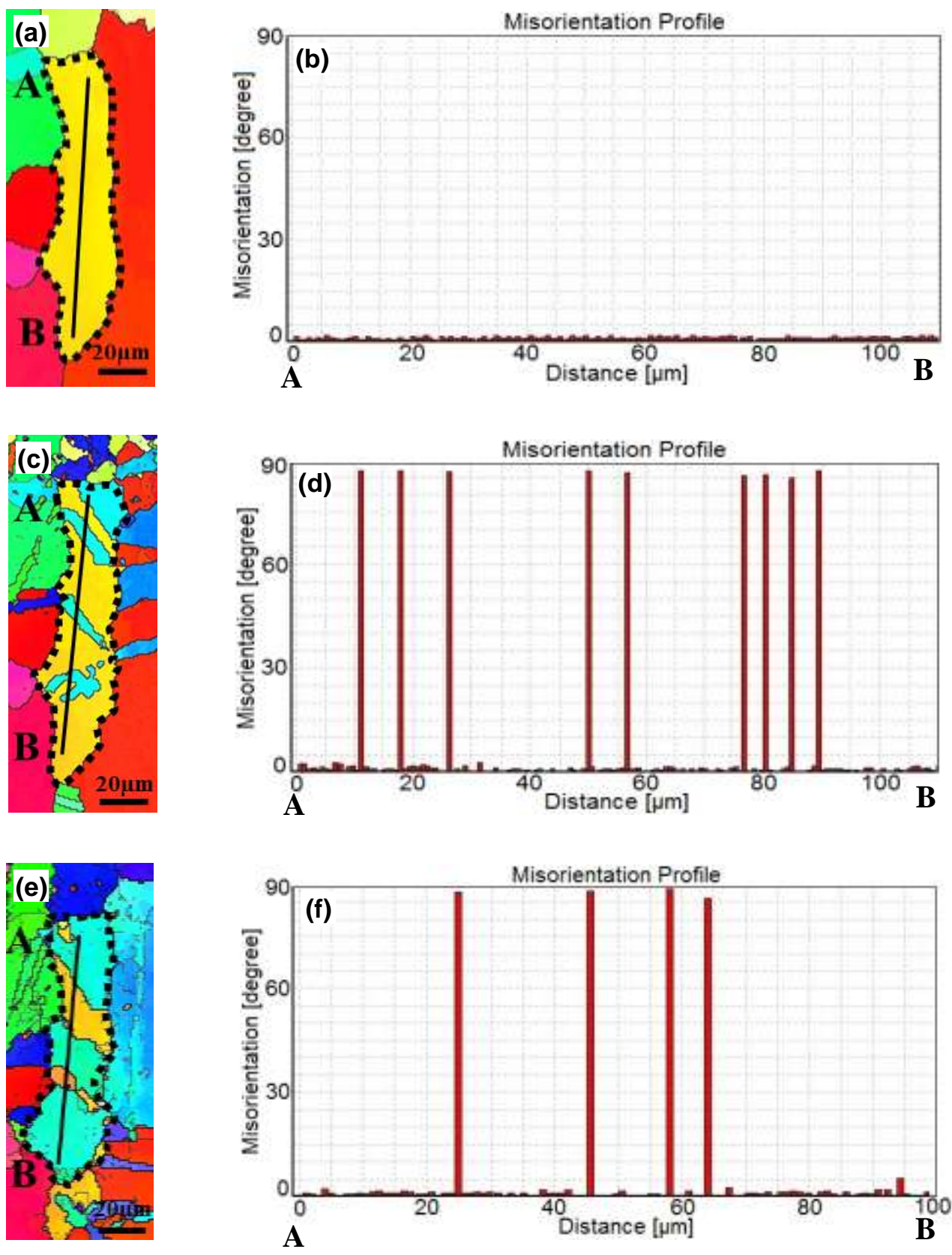

Fig.4.7 Twinning sequence of zone 1- grain 1 by means of characteristic misorientation profiles between points $A$ and $B$ specified on the orientation maps, at true strains of (a, b) $0 \%,(c, d) 2.5 \%$, and (e, f) $4.9 \%$, respectively.

Further compression led to the merging of pre-existing extension twins or the disappearance of twin boundaries, to accommodate the plastic deformation by twinning through the growth of 
prevailing twin lamellae (Fig.4.8(c)). The mechanism of extension twin growth through the nucleation and glide of twinning dislocations on twin boundaries was schematized in Fig.4.8(d).
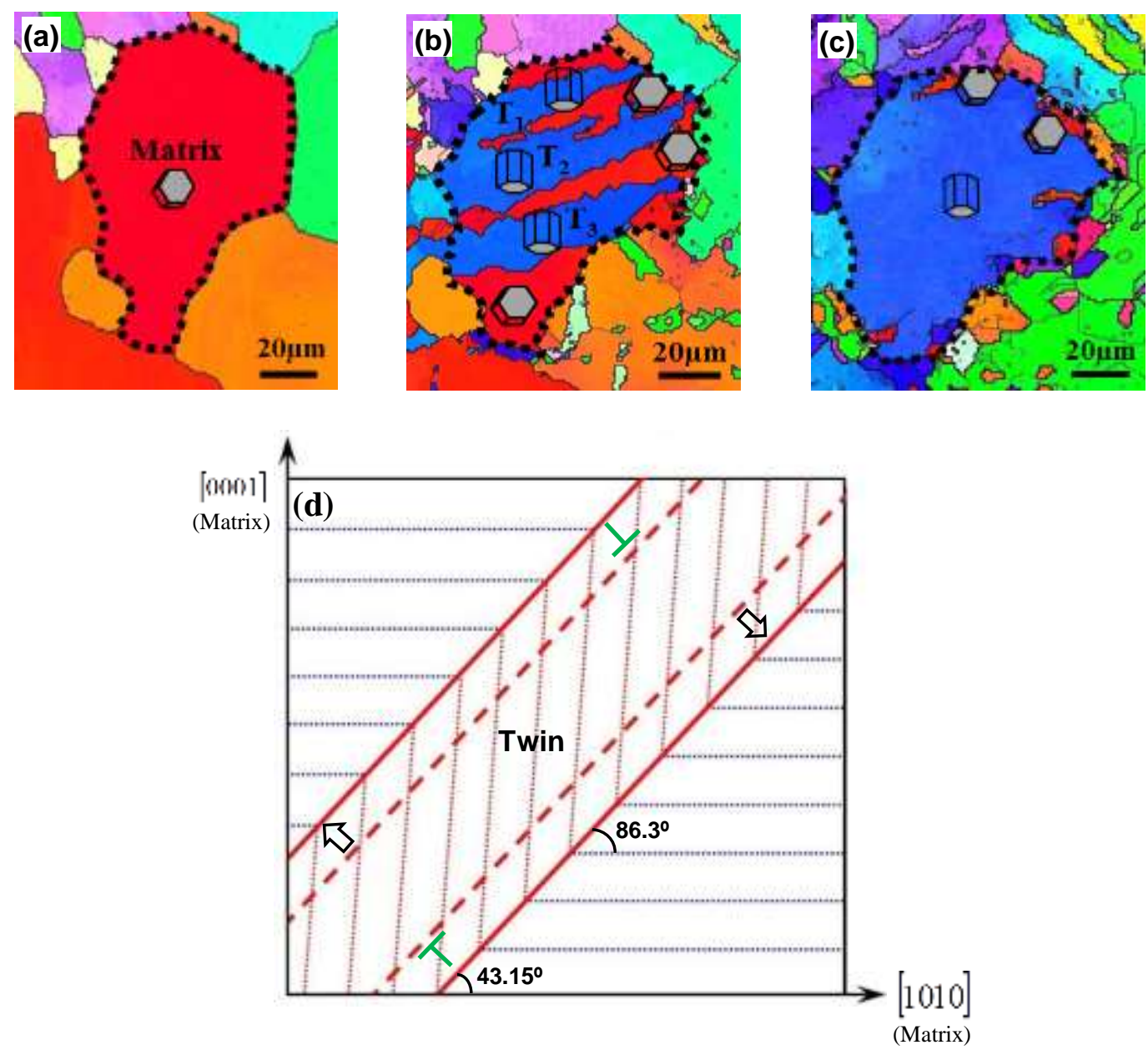

Fig.4.8 $\{10 \overline{1} 2\}$ twin-twin interactions in zone 1 - grain 2: (a) orientation map of the initial twinfree microstructure, and twin growth during stepwise compression of (b) $2.5 \%$ and (c) $4.9 \%$; the lattice orientation is represented by schematic hcp unit cells, and (d) schematic illustration.

The nature of twin growth or twin boundary coalescence in zone 1 - grain 1 (Fig.4.7), and zone 1 - grain 2 (Fig.4.8) was somewhat different. That is, at $\varepsilon=4.9 \%$ zone 1 - grain 2 was almost completely swept by twin growth, while there was still a perceivable untwinned portion left in the zone 1 - grain 1 at $\varepsilon=4.9 \%$, as seen from Fig.4.7(e). This was related to the effect of initial 
grain size, shape and orientation, where he finer the grains and the larger the grain boundary misorientations, the higher the resistance to the nucleation and growth of twins, being similar to the dislocation slip.

\subsubsection{Growth of $\{10 \overline{1} 2\}$ extension twins under similar and distinct twin variants}

Optical image shown in Fig.4.9(a) (i.e., $\mathrm{L}_{2}$ in Fig.4.1(c)) represented twinning deformation through one single variant, where the nucleation of several parallel extension twins with some spacing between them was observed. The lenticular shape of these twins was obvious due to the higher constraint at the intersection of a twin with the grain boundary. This dictated the driving force for the twin to grow near the ends. As a result, extension twins were observed to grow more in the middle area leading to the observed lenticular shapes in Fig.4.9. The $\{10 \overline{1} 2\}$ extension twin in hcp structures possesses six crystallographically equivalent variants. The active twin plane and its trace on the observational surface for a single-variant $\left(V_{l}\right)$ is shown in Fig.1.9(b), through a proper orientation of the hep unit cell with respect to the stress axis. Using EBSD, the evolution of a typical single twin variant could be observed from zone 2 - grain 1 presented in Fig.1.9(c), (d) and (e) through stepwise compression of 0\%, 4.9\% and 6.8\%. Parallel twins were observed to thicken along with the appearance of some new twins with increasing strain, which decreased the spacing between them. The corresponding $\{0001\}$ pole figure (Fig.4.9(f)) displaying the specific orientation of the initial matrix and the twins facilitated the detection of the type and variant of the observed twins in Fig.4.9(d) and (e), where the matrix reoriented towards ED by $\sim 86^{\circ}$, confirming the formation and propagation of extension twins. 


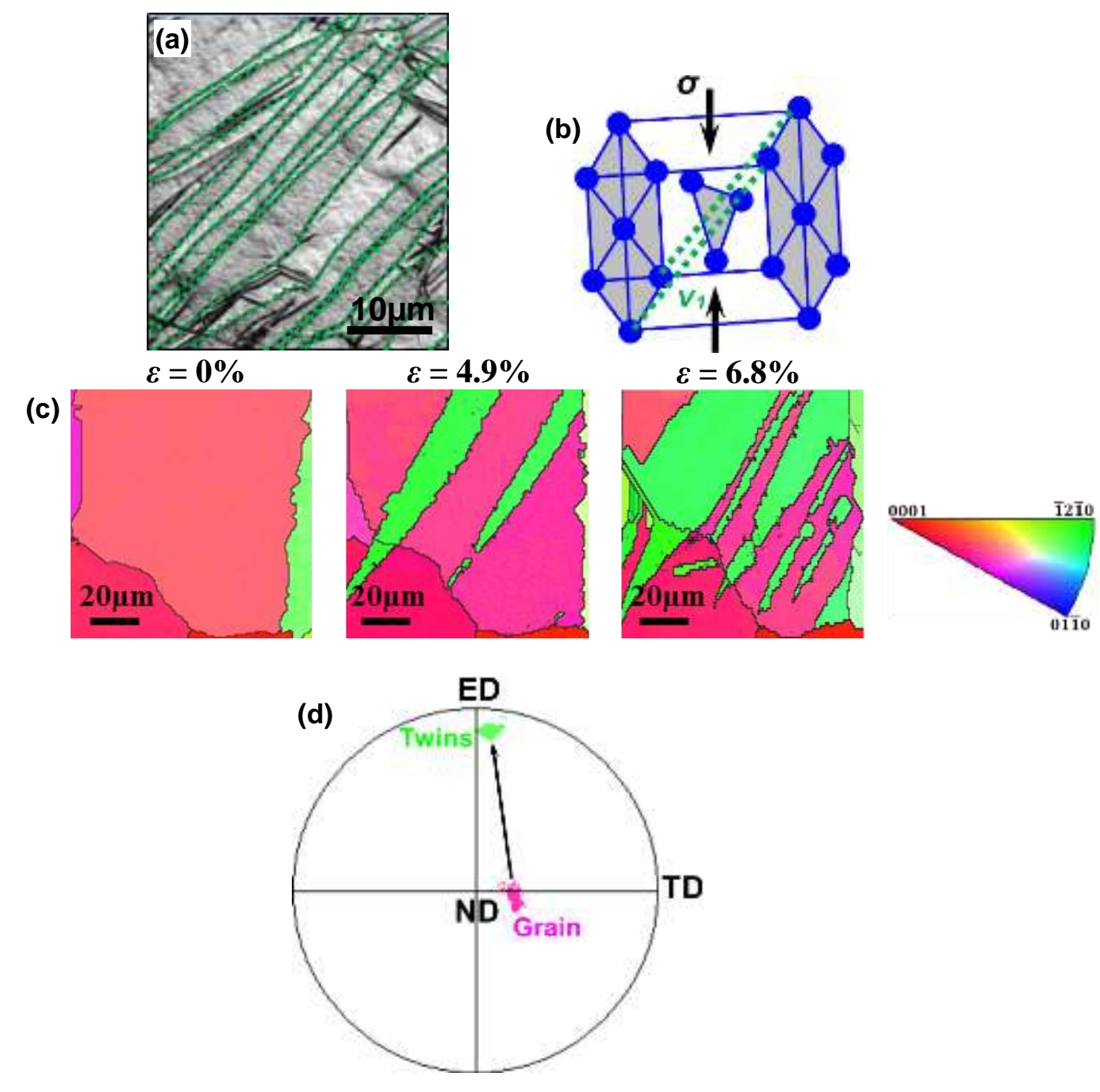

Fig.4.9 (a) Optical micrograph of a grain which experienced one variant of $\{10 \overline{1} 2\}$ extension twinning with (b) the hcp unit cell plotted to detect the active twin planes and their traces. EBSD orientation maps of zone 2 - grain 1 showing the growth of $\{10 \overline{1} 2\}$ twin variant at true strains of (c) $0 \%$, (d) $4.9 \%$ and (e) $6.8 \%$, respectively, and (f) the corresponding $\{0001\}$ pole figure of the matrix and twins.

A different twin morphology with two distinct variants was observed in the optical micrograph of Fig.4.10(a) ( $\mathrm{L}_{3}$ in Fig.1(d)). This phenomenon would involve twin-twin interactions via the growth and inter-blocking between two different twin variants which based on [101] could be characterized as co-zone twins as displayed in the hcp unit cell presented in Fig.4.9(b). It was clearly observed from zone 2 - grain 2 in Fig.4.10(c), (d), and (e) that during compression the 
growth of two variants of twins blocked each other by a "quilted-looking" structure in association with the twin-twin boundary formation.

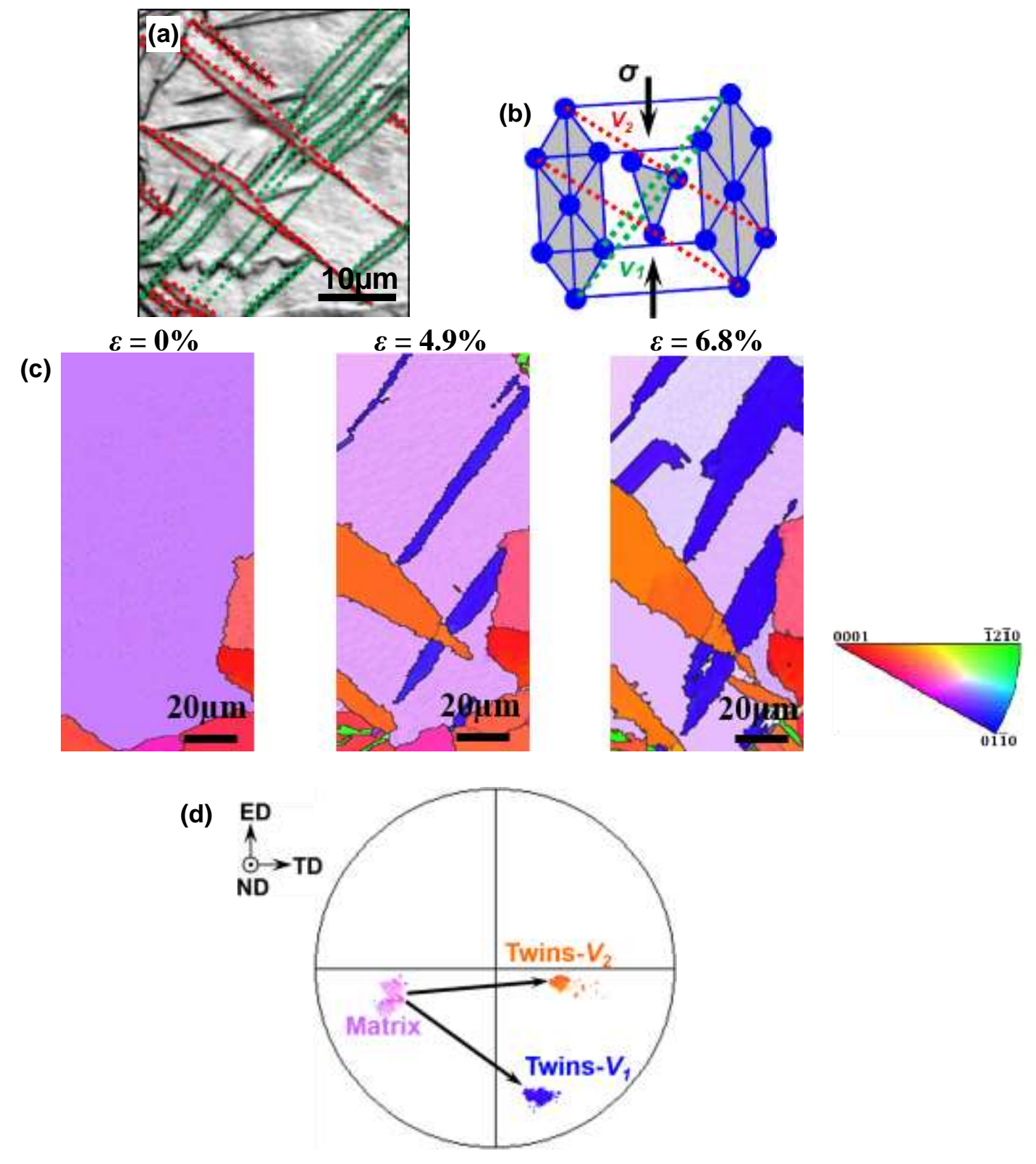

Fig.4.10 (a) Optical micrograph of a grain which experienced two variants of $\{10 \overline{1} 2\}$ extension twinning with (b) the hcp unit cell plotted to detect the active twin planes and their traces. EBSD orientation maps of zone 2 - grain 2 showing the growth of two $\{10 \overline{1} 2\}$ twin variants at true strains of (c) $0 \%$, (d) $4.9 \%$ and (e) $6.8 \%$, respectively, and (f) the corresponding $\{0001\}$ pole figure of the matrix and twins. 
This would retard the twin growth and promote the nucleation of new twins. Although the optical micrograph in Fig.4.10(a) corresponded to a later compression stage ( $\varepsilon=6 \%$ ) in comparison with Fig.9(a) $(\varepsilon=4.3 \%)$, much thinner twins were observed at the same magnification. The matrix region observed in Fig.4.9(a) was nearly saturated at $\varepsilon=4.3 \%$ which indicates that at this stage the nucleation of new twins stopped and instead twin growth became a major deformation mechanism. In this twin growth process, multiple twins of the same variant grew towards a single one until the entire grain was swept via twin merging. In Fig.4.10(a) a roughly counted number of twins was $~ 20$ compared to $~ 10$ twins in Fig.4.9(a), and in the later compressive stage twin boundary coalescence was delayed. It follows that when a grain experiences the propagation of two distinct variants, new twins are observed to nucleate inside the confined spaces created by the several intersections among the different twin variants. The two different twin variants were also identified in Fig.4.10(f) by means of the $\{0001\}$ pole figure displaying the specific orientation of the initial matrix and the two propagating twins. Distinct variants were clearly identified and were observed to convert the matrix orientation to two different axes by an angle of $\sim 86^{\circ}$.

Further investigations were conducted on zone 2 - grain 2 deformed at $6.8 \%$ (Fig.4.10(e)), by considering its Euler angle map (based on the color code provided in Fig.4.11). The matrix M, a few neighboring grains and different twins propagating under similar and different variants were selected in Fig.4.11(a) and were described in terms of their angles in the $3 \mathrm{D}\left(\varphi_{1} \Phi \varphi_{2}\right)$ Euler space. The conversion of $M$ towards $T_{1}$ and $T_{1}$, and towards $T_{2}$ and $T_{2}$ ' was pointed out by the black arrows in Fig.4.11(b). The propagation of the co-zone twins under the two distinct axes from the matrix were clearly seen, which was in agreement with the pole figure in Fig.4.10(f). 
The values of the Euler angles were determined for $\mathrm{M}$ and the selected twins (Fig.4.11(c)) where it was observed that twins sharing the same variant (e.g., $\mathrm{T}_{1}$ and $\mathrm{T}_{1}{ }^{\prime}$ or $\mathrm{T}_{2}$ and $\mathrm{T}_{2}$ ') had almost identical $\left(\varphi_{1} \Phi \varphi_{2}\right)$ triplets. This was expected since extension twins propagating under one single variant were previously seen in Fig.4.9(f) to orient the matrix towards one single new orientation, unlike the case of different twin variants as confirmed by the distribution in the Euler space (Fig.4.11(b)) and the $\left(\varphi_{1} \Phi \varphi_{2}\right)$ angles (Fig.4.11(c)), where a clear re-orientation of the matrix with respect to the co-zone twins was seen.

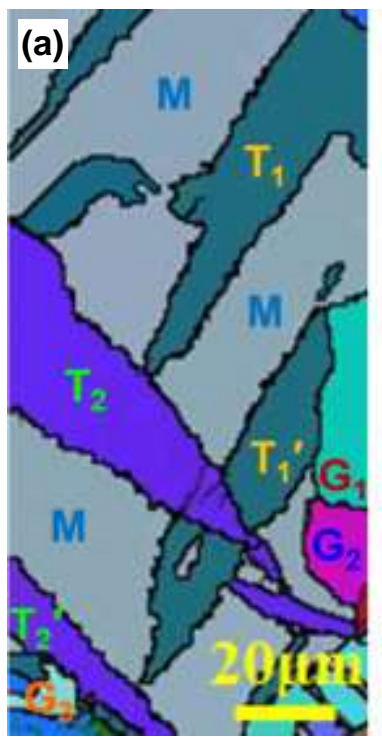

(b)
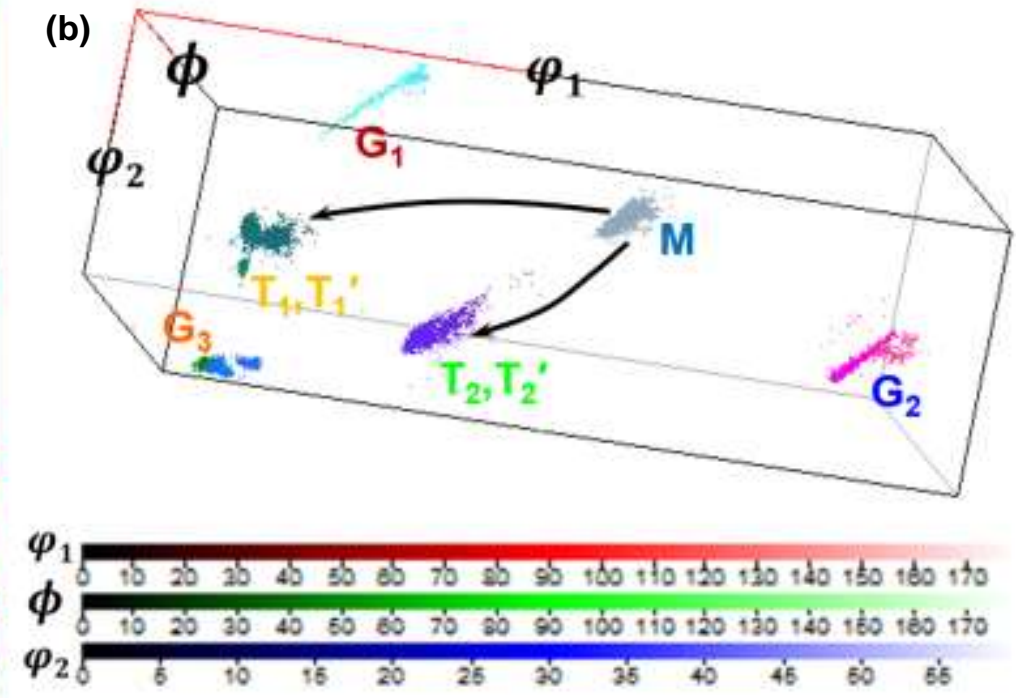

(c)

\begin{tabular}{cccc} 
& $\boldsymbol{\varphi}_{\mathbf{1}}{ }^{\mathbf{o}}$ & $\boldsymbol{\Phi}^{\mathbf{o}}$ & $\boldsymbol{\varphi}_{\mathbf{2}}{ }^{\mathbf{o}}$ \\
\hline $\mathbf{M}$ & 102.34 & 118.61 & 43.89 \\
$\mathbf{T}_{\mathbf{1}}$ & 22.07 & 76.71 & 29.99 \\
$\mathbf{T}_{\mathbf{1}}{ }^{\prime}$ & 22.37 & 77.02 & 29.61 \\
$\mathbf{T}_{\mathbf{2}}$ & 73.12 & 28.68 & 54.17 \\
$\mathbf{T}_{\mathbf{2}}{ }^{\prime}$ & 72.92 & 29.10 & 55.61
\end{tabular}

Fig.4.11 (a) EBSD Euler angle map of zone 2 - grain 2 (Fig.11(e)) strained at $6.8 \%$, (b) orientation relationships between matrix, twins and neighboring grains presented in the $3 \mathrm{D}$ Euler space based on the provided Euler color code, and (c) Euler angles of the matrix and the twins propagating under two different variants. 


\subsubsection{Distinctive characteristics of $\{10 \overline{1} 2\}$ twin-twin interactions}

Although atomistic simulations have been attempted in the literature [103], to the authors' knowledge, specific twin-twin configurations witnessed at different stages of deformation process are lacking. Hence, one major aspect of the present study was to illustrate the kinetics of selected scenarios from the micrographs of Fig.4.1 and the EBSD orientation maps of zone 3 (Fig.4.6) during stepwise compression. Two different twin-twin scenarios have been distinguished and referred to as type A, the ladder-like structure (Fig.4.12) and type B, the branching-like structure (Figs 4.13 and 4.14). Before analyzing these scenarios, it should be noted that the formation process of these twin structures with respect to the compressive stress axis would be more adequate while studying the twin formation in a single crystal. However, in the case of a grain in a polycrystalline material such as the present $\mathrm{AZ31} \mathrm{Mg}$ alloy, the formation of a twin involves shearing of the matrix and when twin nucleation occurs at a grain boundary, the immediate neighborhood in the adjoining grain must also be sheared, and the $2^{\text {nd }}$ order stress state of the grain constrained by neighboring grains has to be considered. Further studies in this aspect are needed.

Fig.4.12(a) represents a ladder-like structure. As shown in the schematic illustration of Fig.4.12(b), it was formed when two pre-existing twins $T_{1}$ and $T_{1}{ }^{\prime}$ with the same $\{10 \overline{1} 2\}$ twin variant were observed to propagate in parallel. Incoming twins $T_{2}, T_{3}$, and $T_{4}$ (i.e., having obviously a smaller size than the pre-existing twins due to their later appearance) were trapped in-between $T_{1}$ and $T_{1}^{\prime}$, leading to a ladder-like characteristic. $T_{2}, T_{3}$, and $T_{4}$ also shared the same variant, but different from $T_{1}$ and $T_{1}{ }^{\prime}$. Due to the lenticular shape of their tips, the new twins $T_{2}$, 
$\mathrm{T}_{3}$ and $\mathrm{T}_{4}$ could not have nucleated from the twin boundaries of the pre-existing twins $\mathrm{T}_{1}$ and $\mathrm{T}_{1}$. Thus, $\mathrm{T}_{2}, \mathrm{~T}_{3}$, and $\mathrm{T}_{4}$ were likely to form separately from the matrix grain as sketched in Fig.4.12(b). It should be noted that for simplification purposes the boundaries of these twins were approximated by two parallel lines rather than the lenticular shape in Fig.4.12(a).

Ladder-like structure was studied via EBSD analysis through stepwise compressions (Fig.4.12(cf)). As stated above, it is confirmed that $T_{1}$ and $T_{1}{ }^{\prime}$ formed first at an earlier compressive stage than that of Fig.4.12(a), since they were already present at a true strain of $1.2 \%$ (Fig.4.12(c)). Then, at $2.5 \%$ (Fig.4.12(d)), a new set of twins with a different variant started to nucleate from the matrix, and impinged on the inner boundaries of $\mathrm{T}_{1}$ and $\mathrm{T}_{1}{ }^{\prime}$, where they were obstructed and twin-twin boundaries formed. This could be more clearly seen at a later compressive stage (Fig.4.12(e) at 5.5\%). At a higher compressive stage of $8 \%$, incoming twins $\mathrm{T}_{2}, \mathrm{~T}_{3}$, and $\mathrm{T}_{4}$ were observed to "dissociate" on the boundaries of pre-existing twins $\mathrm{T}_{1}$ and $\mathrm{T}_{1}$ '. This process was introduced in [102] as the formation of twin-twin boundaries upon dissociating the twinning dislocations of incoming twins into the twinning dislocations of the pre-existing twins with the formation of a residual, leading to the development of a twin "junction" as seen in Fig.4.12(f) where a junction between two twins propagating under a similar variant (i.e., the two merging blue twins in Fig.4.12(f)) was formed along with a pre-existing twin. Hence, the ladder-like structure was characterized by the "trapping" of a series of small parallel twins inside two thicker pre-existing twins, creating the confined spacing bounded by the finer twin lamellas. The orientation relationship between zone 3 - grain 1 and the observed twins in Fig.4.12(c-f) were analyzed by means of the $\{0001\}$ pole figure (Fig.14.12(g)) which displayed the conversion of the matrix orientation towards distinct extension twin variants by $\sim 86^{\circ}$ from the matrix. 
Type A: Ladder-like structure
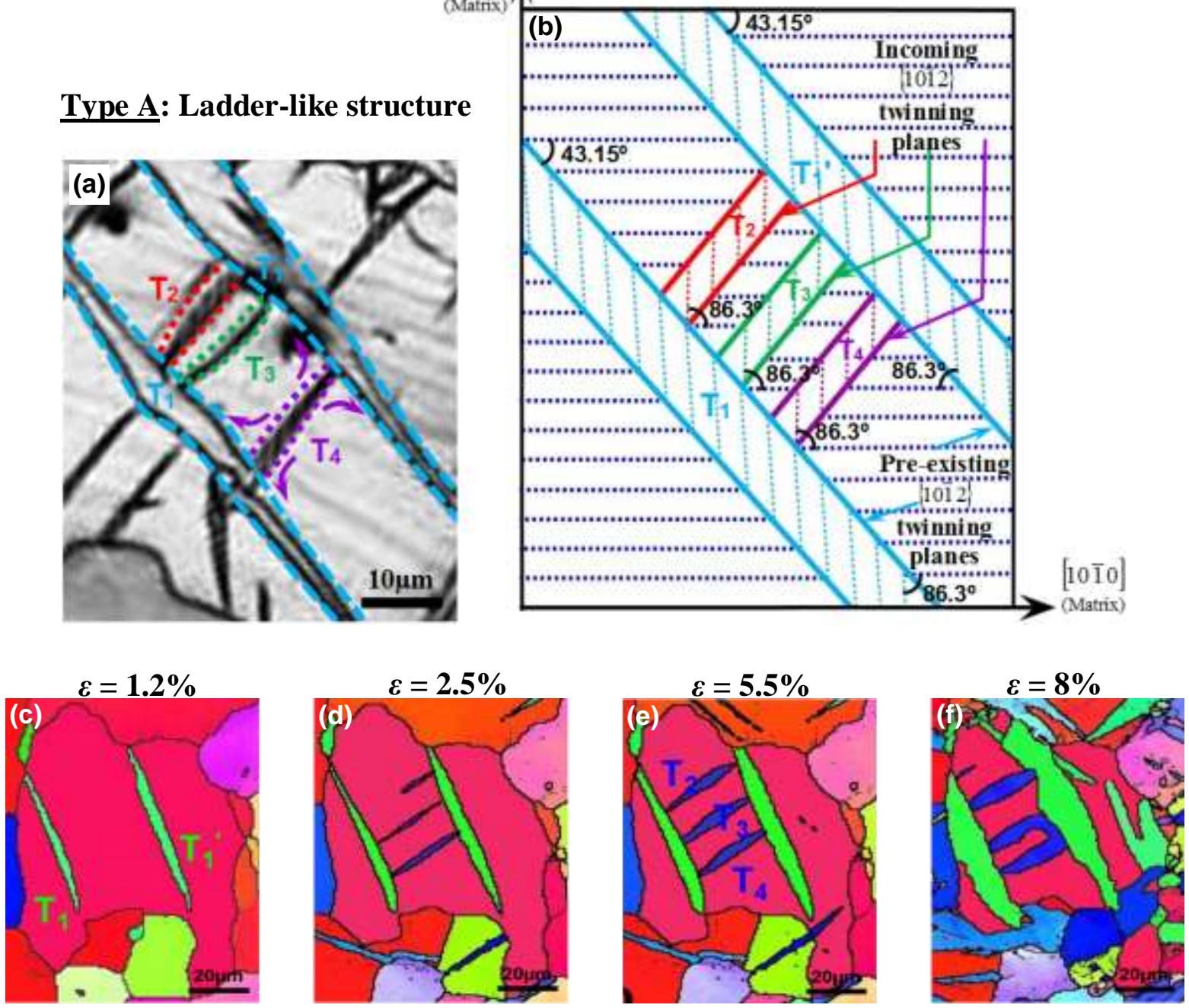

(g)

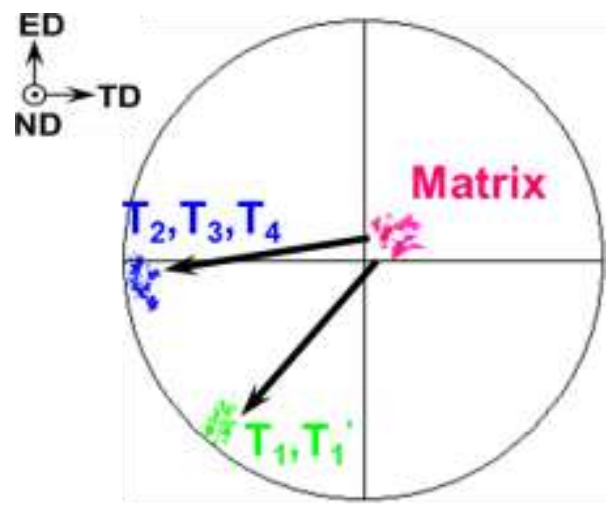

Fig.4.12 (a) Optical micrograph illustrating type A twin-twin interaction (ladder-like structure), and (b) schematic diagram. EBSD orientation maps of zone 3 - grain 1 showing the growth of the ladder-like structure at true strains of (c) $1.2 \%$, (d) $2.5 \%$, (e) $5.5 \%$ and (f) $8 \%$, respectively, and $(\mathrm{g})$ the corresponding $\{0001\}$ pole figure of the matrix and the twins. 
A branching-like structure (Fig.4.13(a)) was also observed at a later compressive stage than the ladder-like structures ( $\mathrm{L}_{6}$ in Fig.4.1(d)). This was likely to be a result of the interactions of three non-co-zone twin variants $V_{1}, V_{2}$, and $V_{3}$ (Fig.4.13(b)). A pre-existing twin $\mathrm{T}_{1}$ (i.e., having the thickest boundaries corresponding to variant $V_{l}$ ) first appeared, and was then followed by incoming twins of the same variant (e.g., $\mathrm{T}_{1}{ }^{\prime}$ and $\mathrm{T}_{1}$ " in Fig.4.13(a)), and of different variants (e.g., $\mathrm{T}_{2}$ and $\mathrm{T}_{2}{ }^{\prime}$ under $V_{2}$, as well as $\mathrm{T}_{3}$ and $\mathrm{T}_{3}{ }^{\prime}$ under $V_{3}$ in Fig.4.13(a)). The shape of the twins in the branching-like structure (e.g., the thickness and sharpness of the tips) was observed to vary among the incoming twins of various variants, which dictated their sequence of appearance. Since the branching-like structure was characterized by the initial formation of $\mathrm{T}_{1}$ (propagating under the variant $V_{l}$ ), and then the appearance of the rest of the twins propagating under $V_{2}$ and $V_{3}$, the Schmid factor (SF) of these variants was of interest in order to understand the mechanisms leading to the selection of specific twin variants. SF was defined as [51],

$$
m=\cos \lambda \cos \phi,
$$

where $\lambda$ is the angle between the load axis and the twinning plane, and $\phi$ is the angle between the load axis and the shear direction. SF values were calculated based on Equ.(4.3) and the detection of the active twin planes and their traces were determined in the HexaSchmid software ( $V_{l}$ in Fig.4.13(c), $V_{2}$ in Fig.4.13(d), and $V_{3}$ in Fig.4.13(e)) for the twins propagating under the various variants involved in Fig.4.13(a). As summarized in Fig.4.13(f), twin $T_{1}$ displayed the highest SF ( 0.499) along the twinning plane and direction of $(10 \overline{1} 2)[10 \overline{1} \overline{1}]$, whereas the rest of the twins propagating under $V_{2}$ along $(\overline{1} 102)[\overline{1} 10 \overline{1}]$ and $V_{3}$ along $(0 \overline{1} 12)[0 \overline{1} 1 \overline{1}]$ displayed a lower Schmid factor $(\sim 0.125)$. The SF values for the different variants thus confirm that in the branching-like structure, the activation and growth of $T_{1}$ were favored by external stresses, when the resolved share stress onto the twinning habit of $V_{l}$ and in the twinning direction is the highest. 


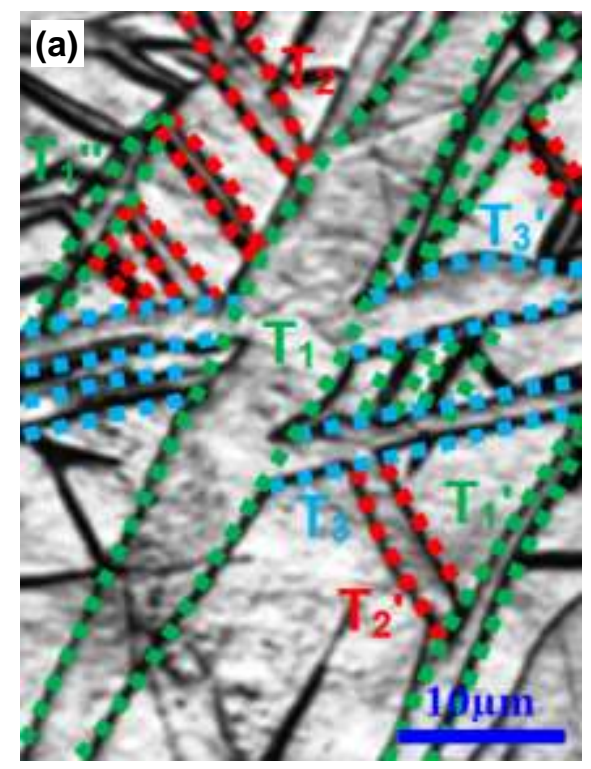

\section{Type B: Branching-like structure}

(b)

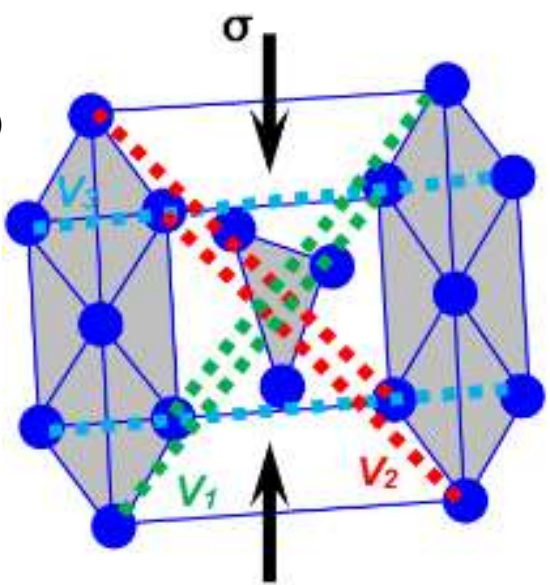

Twin variant $-V_{1}$

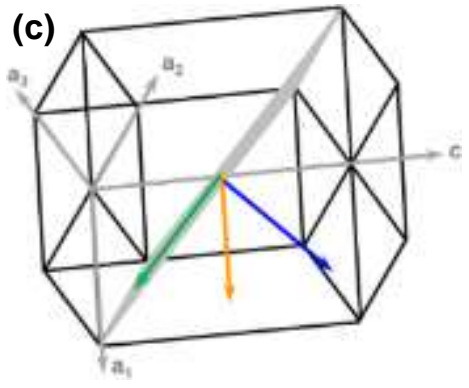

Twin variant $-V_{2}$

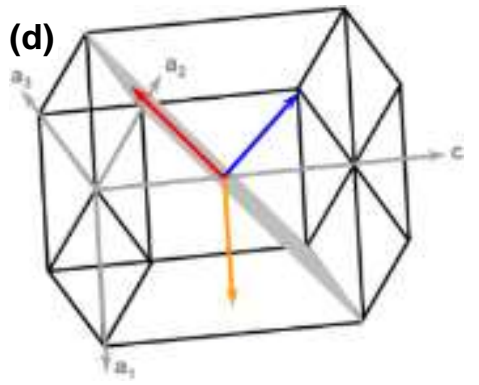

Twin variant $-V_{3}$

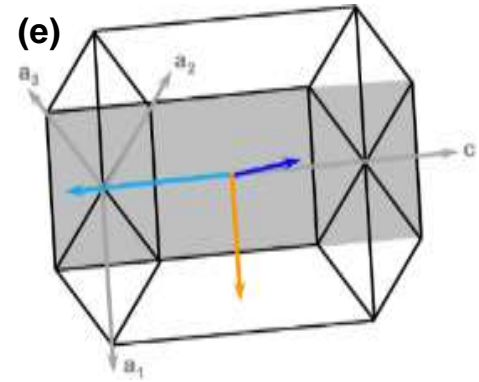

(f)

\begin{tabular}{|ccc|}
\hline & $\begin{array}{c}\text { Twin plane } \\
\text { and direction } \\
(\mathbf{h} \text { k i l })[\mathbf{u} \mathbf{~} \mathbf{t} \text { w] }\end{array}$ & $\begin{array}{c}\text { Schmid factor } \\
\text { (SF) }\end{array}$ \\
\hline$V_{1}$ & $(10 \overline{1} 2)[10 \overline{1} \overline{1}]$ & 0.499 \\
\hline$V_{2}$ & $(\overline{1} 102)[\overline{1} 10 \overline{1}]$ & 0.125 \\
\hline$V_{3}$ & $(0 \overline{1} 12)[0 \overline{1} 1 \overline{1}]$ & 0.125 \\
\hline
\end{tabular}

Load direction

Plane normal

Fig.4.13 (a) Optical micrograph illustrating type B twin-twin interaction (branching-like structure), (b) the corresponding hcp unit cell plotted with respect to the stress axis, and active twin planes and their traces in (c) $V_{1}$, (d) $V_{2}$, and (e) $V_{3}$, and (f) Schmid factors for different variants of extension twinning. 
To depict the evolution of the branching-like structure during stepwise compressions, EBSD investigations were conducted on a selected grain from zone 3 - grain 2, presented in Fig4.14.

(a) $\varepsilon=0 \%$

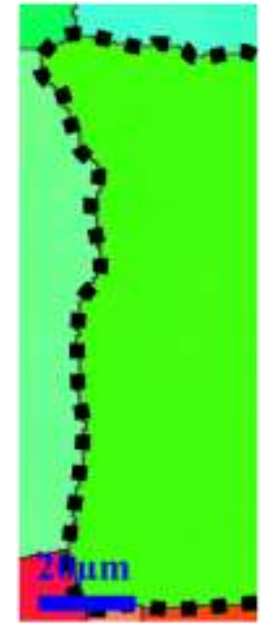

(b) $\varepsilon=1.2 \%$

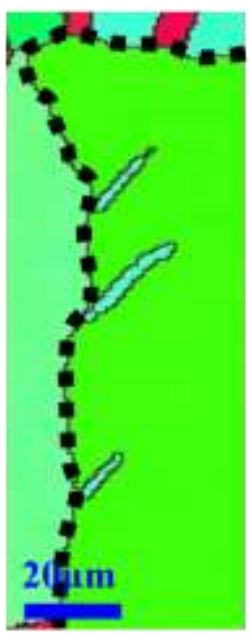

(c) $\varepsilon=2.5 \%$

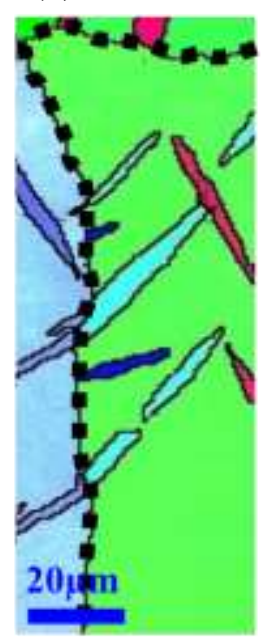

(d) $\varepsilon=5.5 \%$

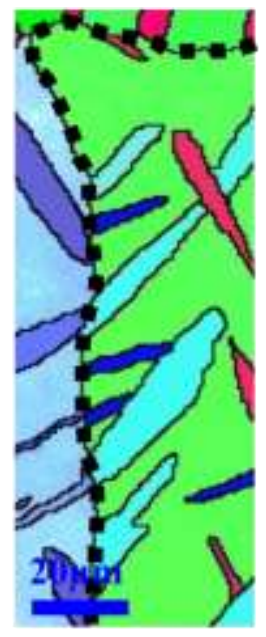

(e) $\varepsilon=8 \%$

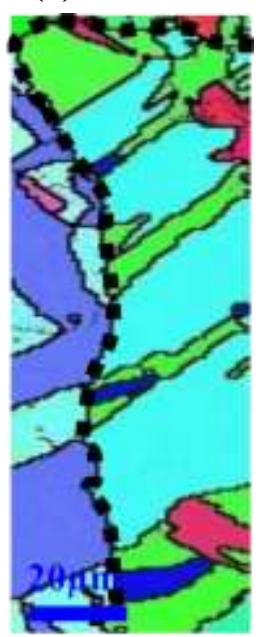

(f)

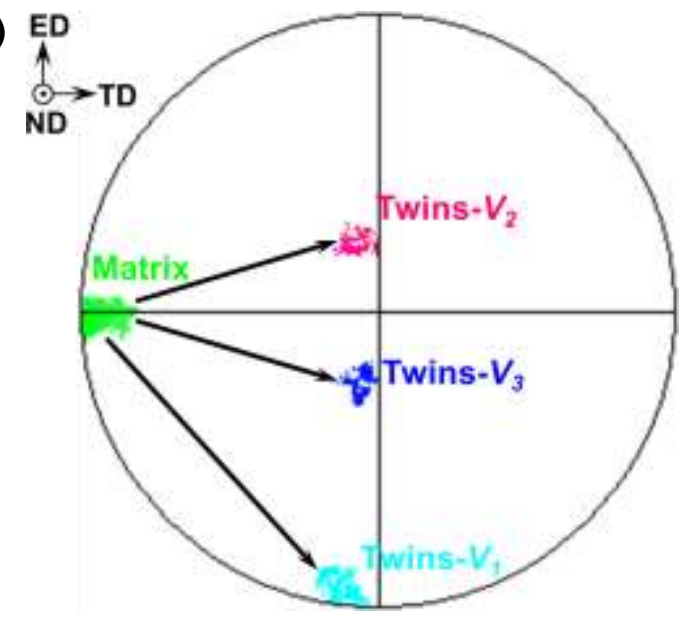

Fig.4.14 EBSD orientation maps showing the growth of the branching-like structure in zone 3 grain 2 at true strains of (a) $0 \%$, (b) $1.2 \%$, (c) $2.5 \%$, (d) $5.5 \%$ and (e) $8 \%$, and (f) the corresponding $\{0001\}$ pole figure of the matrix and the twins.

At the earliest compression stage of $1.2 \%$ (Fig.4.14(b)), zone 3 - grain 2 only witnessed the nucleation of a few thin lenticular twins under the variant $V_{l}$ (Fig.4.13(c)). At the next stage of 2.5\% (Fig.4.14(c)), twins under $V_{l}$ were observed to thicken along with the appearance of two 
sets of twins propagating under $V_{2}$ (Fig.4.13(d)) and $V_{3}$ (Fig.4.13(e)). At 5.5\% (Fig.4.14(d)), all twins under the three distinct variants continued to grow, however twins under $V_{l}$ were characterized by a thicker morphology. The results observed in Fig.4.14(d) at 5.5\% confirmed the previous findings discussed in Fig.4.13(a) due to the proximity of their true strain values, since Fig.4.13(a) was depicted at $6 \%$ true strain. At the final compressive stage of $8 \%$, twins propagating under $V_{l}$ were observed to be highly active compared to the twins growing along the other two variants. Thus, the EBSD analysis was in agreement with the SF results where $V_{l}$ displayed the largest SF of $\sim 0.499$ (Fig.4.13(f)), being favored by the external stresses. In addition, the $\{0001\}$ pole figures of zone 3 - grain 2 and the twins propagating during deformation were displayed in Fig.4.14(f). The matrix grain orientation was observed to change into three distinct orientation axes up to $>85^{\circ}$, corroborating the presence of three variants of $\{10 \overline{1} 2\}$ extension twins. In this context, Godet et al. [51] established a schematic representation of the $\{0001\}$ pole figure of the orientations obtained by extension twinning along with their respective SFs and the obtained results are highly consistent with the variant locations on the pole figure in Fig.4.14(f) and the calculated SFs in Fig.4.13(f).

\subsubsection{Re-twinning and double twinning}

Fig.4.15(a) presents a micrograph of a double extension twin structure where the same variant of secondary $\{10 \overline{1} 2\}$ twin formed inside the primary extension twin. As shown in the schematic diagram (Fig.4.15(b)), re-twinning (i.e., double twinning inside the pre-existing twin with the same twin variant as the parent twin), was observed. In this case both active twin variants were identical and the secondary twin had its basal planes parallel to those of the untwinned matrix. 
Fig.4.15(c) represents zone 2 - grain 3 undergoing stepwise compressions which was observed to experience the nucleation and growth of a single twin $\mathrm{T}_{1}$ at $4.9 \%$ (Fig.4.15(d)). At a more advanced compression of $6.8 \%$ (Fig.4.15(e)), $\mathrm{T}_{1}$ was observed to thicken considerably in comparison with Fig.4.15(d), and a thin twin $\left(\mathrm{T}_{2}\right.$ in Fig.4.15(e)) was observed to propagate under the same variant as the parent twin $\mathrm{T}_{1}$. To better understand the twinning sequence of Fig.4.15(e), the $\{0001\}$ pole figure of the matrix and the twins was presented in Fig.4.15(f). It demonstrates that the initial twinning of $\mathrm{T}_{1}$ converted the matrix orientation to $\sim 86^{\circ}$ towards $\mathrm{ED}$, then the further strain changed the orientation of $\mathrm{T}_{1}$ to $\mathrm{T}_{2}$ by extension twinning as well $\left(\sim 86^{\circ}\right)$ around the same axis. After this double twinning sequence, the scattered orientation went nearly back to the basal orientation. This would be expected since double twinning with the same variant as the parent twin was observed in the schematic representation of Fig.4.15(b) to lead back to a secondary $\{10 \overline{1} 2\}$ twinning basal plane. The nature of the observed double twin was also revealed through the peaks of the misorientation profiles which occurred when the boundaries of $\mathrm{T}_{1}$ (Fig.4.15(g)) and $\mathrm{T}_{2}$ (Fig.4.15(h)) were crossed. It is clear that misorientation angles of $\sim 86.3^{\circ}$ were obtained in all four crossings, confirming the presence of the same variant $\{10 \overline{1} 2\}$ double twinning.

One of the most common double twins is the $\{10 \overline{1} 3\}-\{10 \overline{1} 2\}$ combination shown in Fig.4.16(a). Based on Kwon et al. [104], the $\{10 \overline{1} 3\}-\{10 \overline{1} 2\}$ double twin is usually generated by the formation of a secondary $\left\{10 \overline{1}_{2}\right\}$ twin inside a primary $\{10 \overline{1} 3\}$ twin. The formation sequence of the double twin could be debated. The first possibility is that the $\{10 \overline{1} 2\}$ secondary extension twin occurs simultaneously during growth of the primary contraction twin to facilitate the complex shuffle required at the tip of the growing contraction twin. 

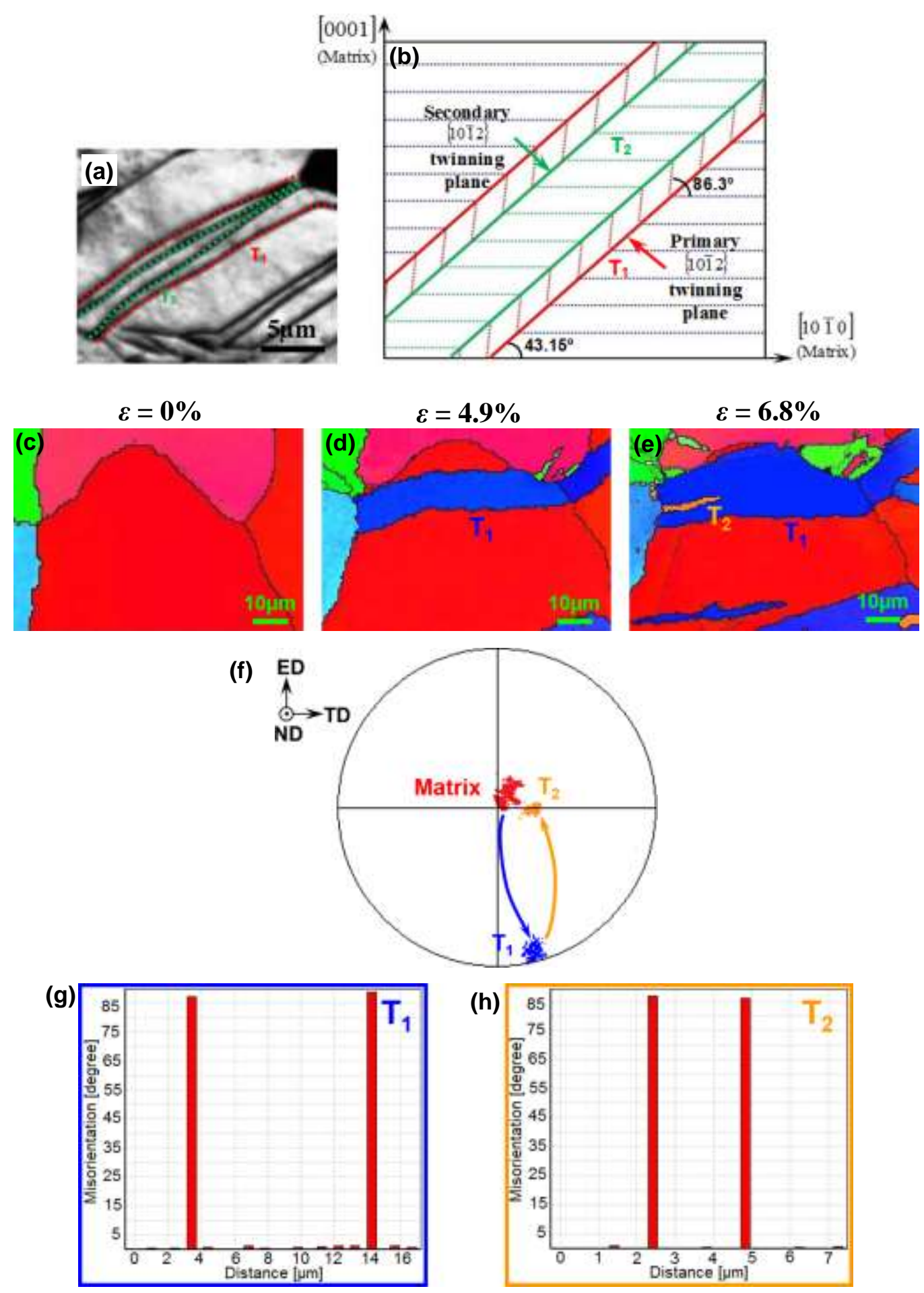

Fig.4.15 (a) Optical micrograph of a grain which experienced $\{10 \overline{1} 2\}$ double twinning with the same twin variant as the parent twin, and (b) the corresponding schematic illustration. EBSD orientation maps of zone 2 - grain 3 at true strains of (c) $0 \%$, (d) $4.9 \%$ and (e) $6.8 \%$, respectively, (d) the corresponding $\{0001\}$ pole figure of the matrix and twins, and misorientation profiles across the boundaries of $(\mathrm{g})$ the parent twin $\mathrm{T}_{1}$, and $(\mathrm{h})$ the secondary twin $\mathrm{T}_{2}$. 
The second contrasting possibility is that despite their narrow size, contraction twins are assumed to form first and then re-twin on $\left\{10 \overline{1}_{2}\right\}$ thus supporting the delayed secondary twinning hypothesis. Beyerlein et al. [54] reinforced the second hypothesis by proving that due to the occurrence of dissociation reactions of slip dislocations, the primary $\{10 \overline{1} 3\}$ twin domain thickens sufficiently to incorporate the $\{10 \overline{1} 2\}$ secondary extension twin, and to contribute to strain accommodations. A schematic illustration attempting to represent the $\{10 \overline{1} 3\}-\{10 \overline{1} 2\}$ double twin could be sketched in Fig.4.16(b), where a pre-existing or newly nucleated $\{10 \overline{1} 3\}$ contraction twin ( $64^{\circ}$ in misorientation) was assumed to quickly retwin with a $\{10 \overline{1} 2\}$ variant, leading to the double twin which continued to grow into the matrix grain. Further evidence could be provided from the EBSD analysis of zone $3-\mathrm{P}_{1}$ (Fig.4.16(c)). At a true strain of $5.5 \%$, only the untwinned area of the matrix grain was observed. At the next compressive stage of $8 \%$, a double twin structure of very fine twin lamellas nucleated. To better understand the twinning sequence, the $\{0001\}$ pole figure of the matrix and the twins was provided. The sequence of twinning could be traced from the matrix orientation which was observed to travel towards $T_{1}$ after undergoing an angle $>60^{\circ}$. $T_{1}$ was then observed to re-orient towards $T_{2}$ by $\sim 86^{\circ}$. Similar observations were made in [55] while studying the formation sequence of the analogous $\{10 \overline{1} 1\}-\{10 \overline{1} 2\}$ double twin where a primary $\{10 \overline{1} 1\}$ twins were followed by $\{10 \overline{1} 2\}$ twins contained within the $\{10 \overline{1} 1\}$.

Another type of twinning was depicted in zone 3 - grain 3 (Fig.4.17(a)) starting from early compression stages at $\varepsilon=1.2 \%$, where a few thin twin lamellas were observed (e.g., $\mathrm{T}_{1}$ in Fig.4.17(b)). To understand the nature of these twins, the corresponding discrete $\{0001\}$ pole figure of the matrix and the twins was displayed in Fig.4.17(f) where a re-orientation by an angle $>70^{\circ}$ was observed. 


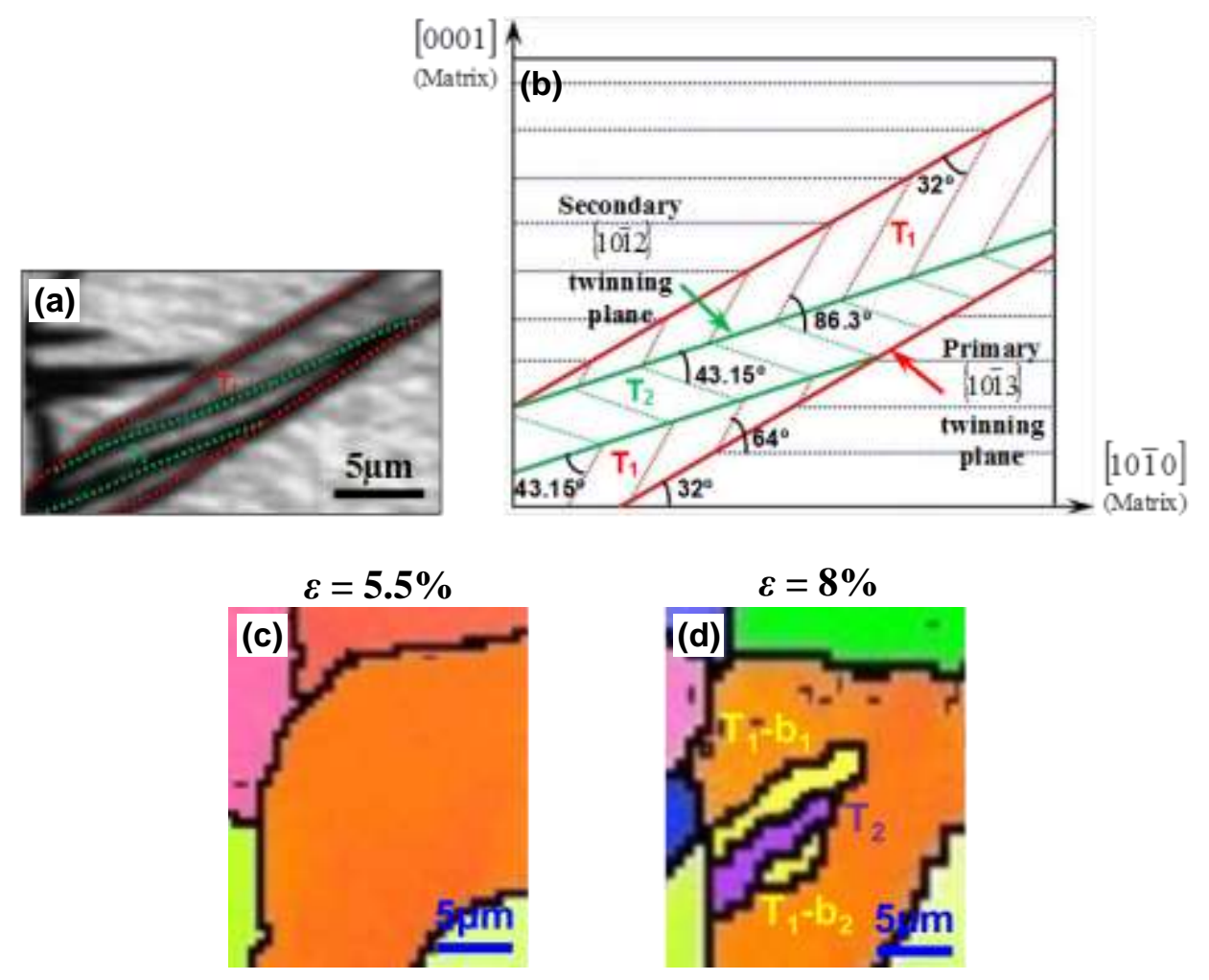

(e)
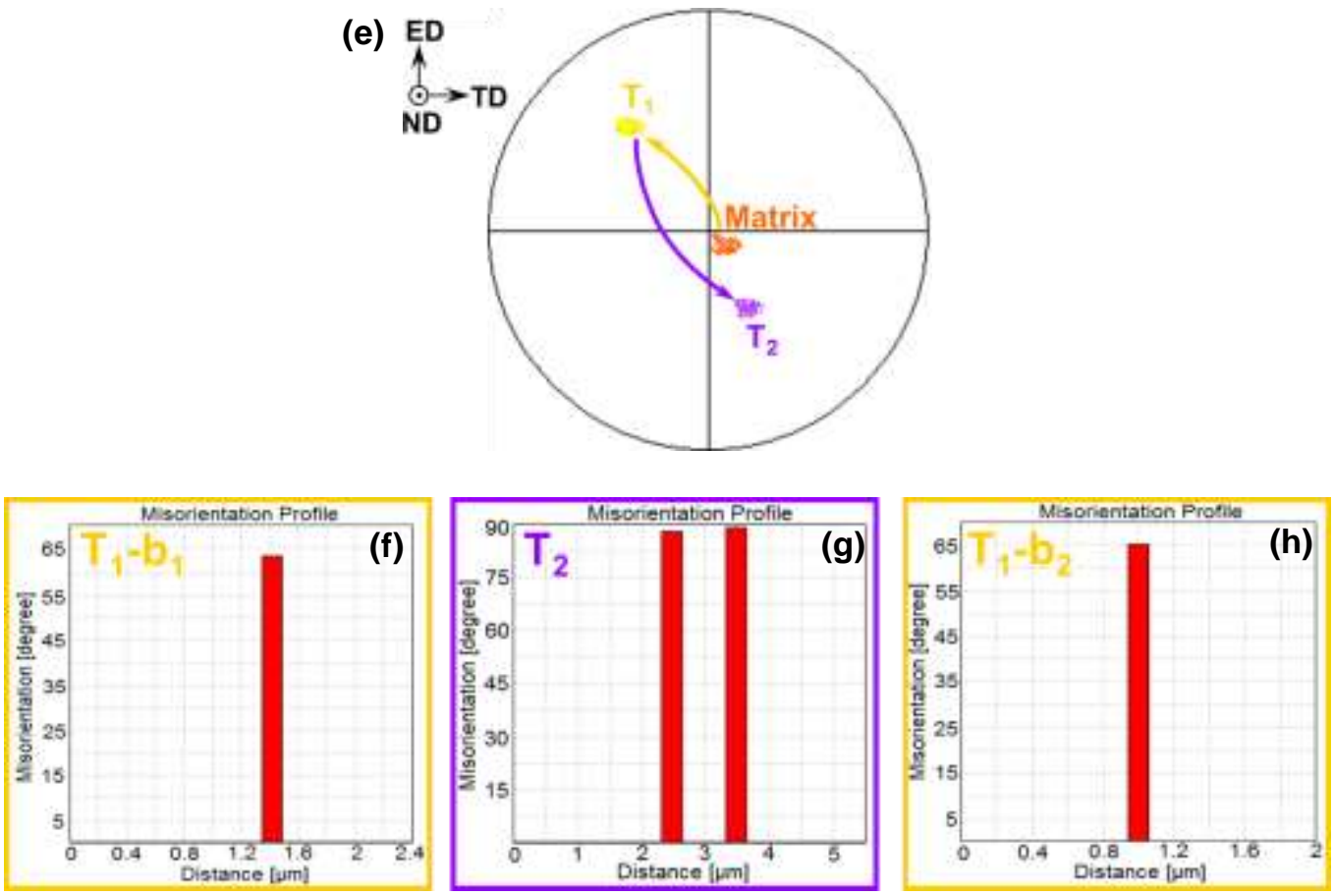

Fig.4.16 (a) Optical micrograph of a grain which experienced $\{10 \overline{1} 3\}-\{10 \overline{1} 2\}$ double twin and (b) the corresponding schematic illustration. EBSD orientation maps of zone $3-\mathrm{P}_{1}$ (Fig.7(e)) compressed at (c) $5.5 \%$, and (d) $8 \%$, showing the formation of the $\{10 \overline{1} 3\}-\{10 \overline{1} 2\}$ double twin, which is confirmed through (e) the corresponding $\{0001\}$ pole figure of the matrix and the twins, and the misorientation profiles across the boundaries (f) $b_{1}$ and $(h) b_{2}$ of the parent twin $T_{1}$ and $(g)$ the secondary twin $T_{2}$. 
This corresponded to the $\{11 \overline{2} 1\}$ twin referred to as the extension twin embryo, and inducing a rotation of the twinning plane by $\sim 72.9^{\circ}$ with respect to the matrix basal plane [105]. Wang et al. [106] studied the nucleation of twin embryos in BCC structures and stated that twin embryo was always able to nucleate from the free surface and to expand into a twin band in some favorable surface sites. Extension twin embryo has also been reported in the literature as a mechanism that aided the formation of $\{10 \overline{1} 2\}$ extension twins. Hence, to understand better the evolution of extension twin embryos, zone 3 - grain 3 was compressed further at 2.5\% (Fig.4.17(c)). The first observation that could be made is that the orientation of the parent matrix and the twin embryos in Fig.4.17(c) slightly varied compared to those shown in Fig.4.17(a) and (b). This could be attributed to the appearance of several lenticular twins under different variants (e.g., $T_{2}, T_{3}$ and $\mathrm{T}_{4}$ in Fig.4.17(c)). Since new twin boundaries were generated, and as the twins started to grow, slip in the parent was detected, causing the formation of local recessions and leading to the observed orientation change in both parent, twins, and a few neighboring grains. The analysis of the twinning features in Fig.4.17(c) revealed that the pre-existing $\{11 \overline{2} 1\}$ twins started already to endure several secondary $\left\{10 \overline{1}_{2}\right\}$ twins at $2.5 \%$ (e.g., formation and propagation of $\mathrm{T}_{2}$ within $\mathrm{T}_{1}$ in Fig.4.17(c)). In addition, a few single $\{10 \overline{1} 2\}$ twins were observed to propagate individually from the matrix under different variants (e.g., $\mathrm{T}_{3}$ in Fig.4.17(c)). It is noted that the pink twin variant which also started to appear (e.g., $\mathrm{T}_{4}$ in Fig.4.17(c)) was not taken into consideration since only the twins which interacted with the extension twin embryos were of interest. The corresponding \{0001\} discrete pole figure (Fig.4.17(g)) revealed different possible orientation scenarios: (1) The matrix travels by $\sim 70^{\circ}$ towards the $\{11 \overline{2} 1\}$ orientations, which re-orients again by $\sim 86.3^{\circ}$, leading to the formation of $\{10 \overline{1} 2\}$ extension twins (the nucleation of $T_{2}$ within $T_{1}$ could be taken as an example). (2) The matrix travels by $\sim 86^{\circ}$ towards the $\{10 \overline{1} 2\}$, leading to the formation of 
single $\{10 \overline{1} 2\}$ extension twins (the nucleation of $\mathrm{T}_{3}$ in the parent matrix of Fig.4.17(c) could be taken as an example). It is noted that both scenarios (1) and (2) are possible since the embryonic twin structures are only present to facilitate the propagation of $\{10 \overline{1} 2\}$ twins and do not oppose their single propagation. At a further compressive stage of $5.5 \%,\{11 \overline{2} 1\}$ twins were less seen and started to vanish to the expense of the $\left\{10 \overline{1}_{2}\right\}$ extension twins. This could be related to the merging of the individual $\{10 \overline{1} 2\}$ twins propagating within the parent grain and the secondary $\{10 \overline{1} 2\}$ extension twins formed inside the $\{11 \overline{2} 1\}$ embryos, which would lead to the disappearance of the embryos. The scattered pole figure in Fig.4.17(h) showed a decrease in the distribution of the $\{11 \overline{2} 1\}$ twins compared to Fig.4.17(g) (i.e., less orange color observed towards the center), however an increase in the scattered points of the $\{10 \overline{1} 2\}$ extension twins was noted in Fig.4.17(h) (i.e., the darker blue color towards ED). The scatter points of the matrix (light blue color towards TD) also decreased in Fig.4.17(h) since the grain is extensively twinned by the $\{10 \overline{1} 2\}$ twins. The final compressive stage at $8 \%$ confirmed all the previous observations since the embryonic twins were almost invisible in Fig.4.17(e) and only thin dots were observed at the center in the Fig.4.17(j). The matrix was also highly twinned due to the $\{10 \overline{1} 2\}$ twin boundary coalescence and the corresponding poles representing the matrix also decreased in Fig.4.17(j), however an increasing number of scattered poles were formed towards ED, confirming the basal plane rotations due to extension twinning. The thick red dashed arrow in Fig.4.17(j) shows that at a true strain of $8 \%$, the $\{11 \overline{2} 1\}$ embryonic twins which played an important role of a "connecting bridge" at early compressive stages between the matrix and the twins almost all vanished, and the matrix is assumed to twin directly by $\sim 86^{\circ}$, without the intermediate passage by the $\{11 \overline{2} 1\}$ twins. 
(a) $\varepsilon=0 \%$

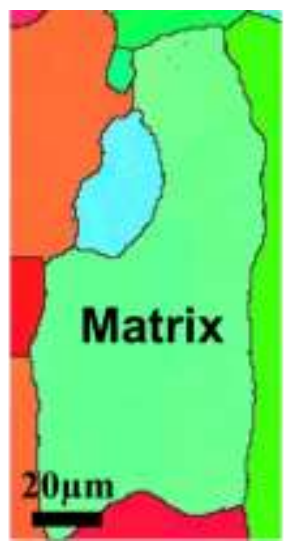

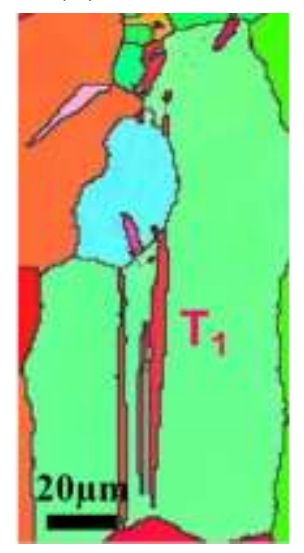

(c) $\varepsilon=2.5 \%$

(d) $\varepsilon=5.5 \%$

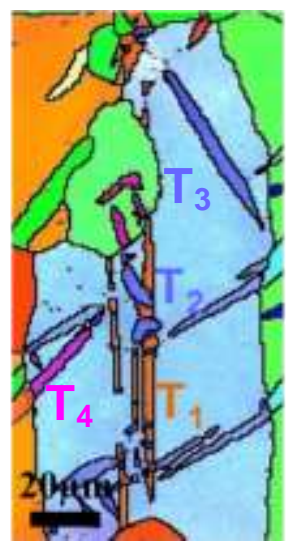

(e) $\varepsilon=\mathbf{8 \%}$
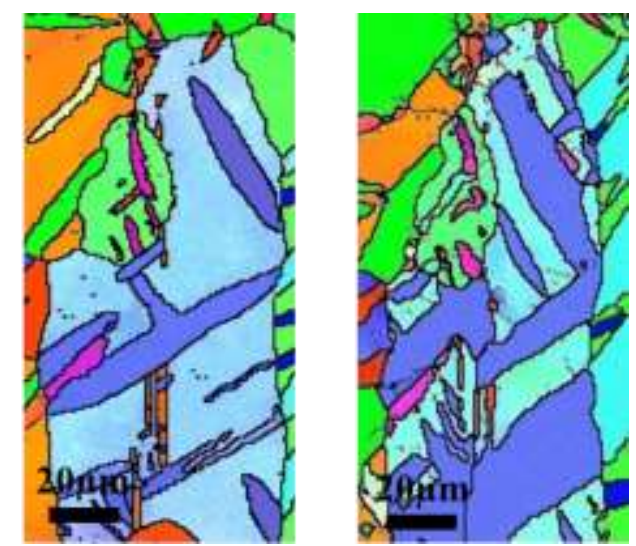
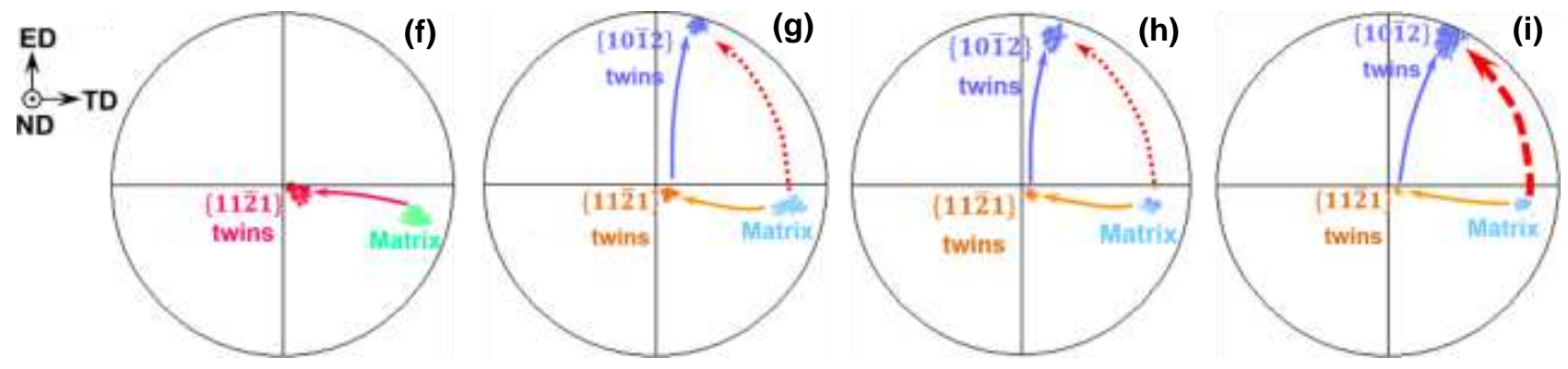

Fig.4.17 (a) Initial EBSD orientation map of zone 3 - grain 3, along with the deformed maps (observational plane and color legend displayed in Fig.4) and their corresponding $\{0001\}$ pole figures of the matrix and the twins at true strain values of $(b, f) 1.2 \%,(c, g) 2.5 \%,(d, h) 5.5 \%$, and (e, i) $8 \%$.

\subsubsection{Feasibility of crossing between different types of twins}

In this section the possibility of the transmission of an impinging twin is examined when crossing another twin through a secondary twinning path. Fig.4.18(a) displays zone $3-\mathrm{P}_{2}$ at a compressive stage of $5.5 \%$ where a few lenticular extension twins were observed to propagate. The same location was observed at a later compressive strain (Fig.4.18(b) at $8 \%$ ) where the previously observed extension twins merged $\left(\mathrm{T}_{\mathrm{e} 1}\right.$ and $\mathrm{T}_{\mathrm{e} 2}$ in Fig.4.18(b)) and a very thin $\{10 \overline{1} 1\}$ contraction twin $\left(\mathrm{T}_{\mathrm{c}}\right.$ in Fig.4.18(b)) was detected between them. 

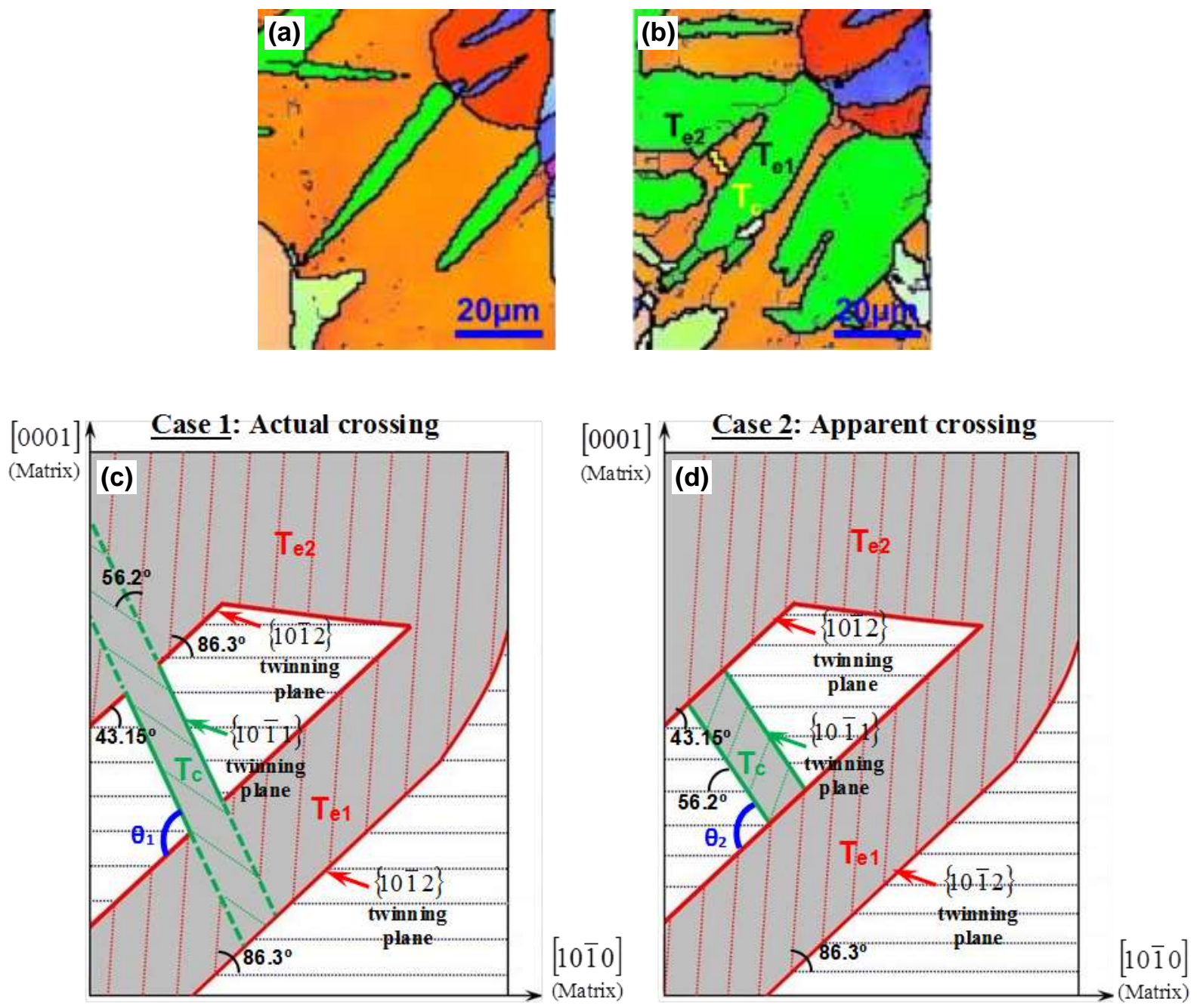

Fig.4.18 EBSD orientation maps of zone $3-\mathrm{P}_{2}$ compressed at (a) $5.5 \%$ and (b) $8 \%$, showing the formation of a crossing-like twin structure between a thin $\{10 \overline{1} 1\}$ contraction twin which encounters two merged $\{10 \overline{1} 2\}$ extension twins. Schematic illustrations of (c) the case of an actual crossing and (d) the case of an apparent crossing between twins are also provided.

The feature observed in Fig.4.18(b) was considered as an "apparent crossing" twin structure, where impinging twins were linked on each side of twin lamella. To better illustrate this behavior, microstructural characteristics were compared with two schematic scenarios (Fig.4.18(c) and (d)), where the angle $\theta$ in Fig.4.18(b) was of concern. The detailed sketches revealed that $\theta<\theta_{1}$ (Fig.4.18(c)) in the case of an actual crossing (i.e., the twin basal plane of $\{10 \overline{1} 1\}$ was obtained 
through a misorientation of $\sim 56.2^{\circ}$ of the $\{10 \overline{1} 2\}$ twin basal plane on both sides of the crossing), where the narrow banded contraction twin was observed to pass through the merged extension twins. In the case of Fig.4.18(d), $\theta \sim \theta_{2}$ and it could be seen that the twinned crystal encountered two extension twin boundaries crossing the secondary twinning path of the contraction twin; however, none of twins was actually crossing the other (i.e., $\mathrm{T}_{\mathrm{c}}, \mathrm{T}_{\mathrm{e} 1}$ and $\mathrm{T}_{\mathrm{e} 2}$ were linked on the sides of the twin lamellas where $T_{c}$ was obtained by a crystal orientation of $\sim 56.2^{\circ}$ from the matrix basal plane). Yu et al. [102] studied the twin intersection region as well as the $c$-axis misorientation with respect to the crystal orientation and concluded that no twin crossing or transmission occurred upon the contact of one twin with another twin; only a "tilt" was induced by dislocation formation at the twin-twin boundary. The previous statement could be related to the fact that hcp structures were characterized by unidirectional twinning having one set of dislocations per twinning plane, hence the unlikeliness of a crossing twin structure.

\subsubsection{Theoretical analysis of twin-twin interactions}

As previously seen, when a grain experiences single or double variants of $\{10 \overline{1} 2\}$ extensions twinning, twin-twin boundaries are expected to form due to the interaction of two or more twin variants with each other. In this context, Fig.4.19(a) represents two incoming twins $\mathrm{T}_{2}$ and $\mathrm{T}_{3}$ of

the same $\{10 \overline{1} 2\}$ twin variant, approaching the boundary of twin $\mathrm{T}_{1}$ having a different $\{10 \overline{1} 2\}$ twin variant. $T_{1}$ is referred to as the pre-existing twin; $T_{2}$ and $T_{3}$ as the incoming twins, based on their chronological appearance. The nature of twinning dislocation reaction was a key in differentiating the three mechanisms presented in Fig.4.19. 

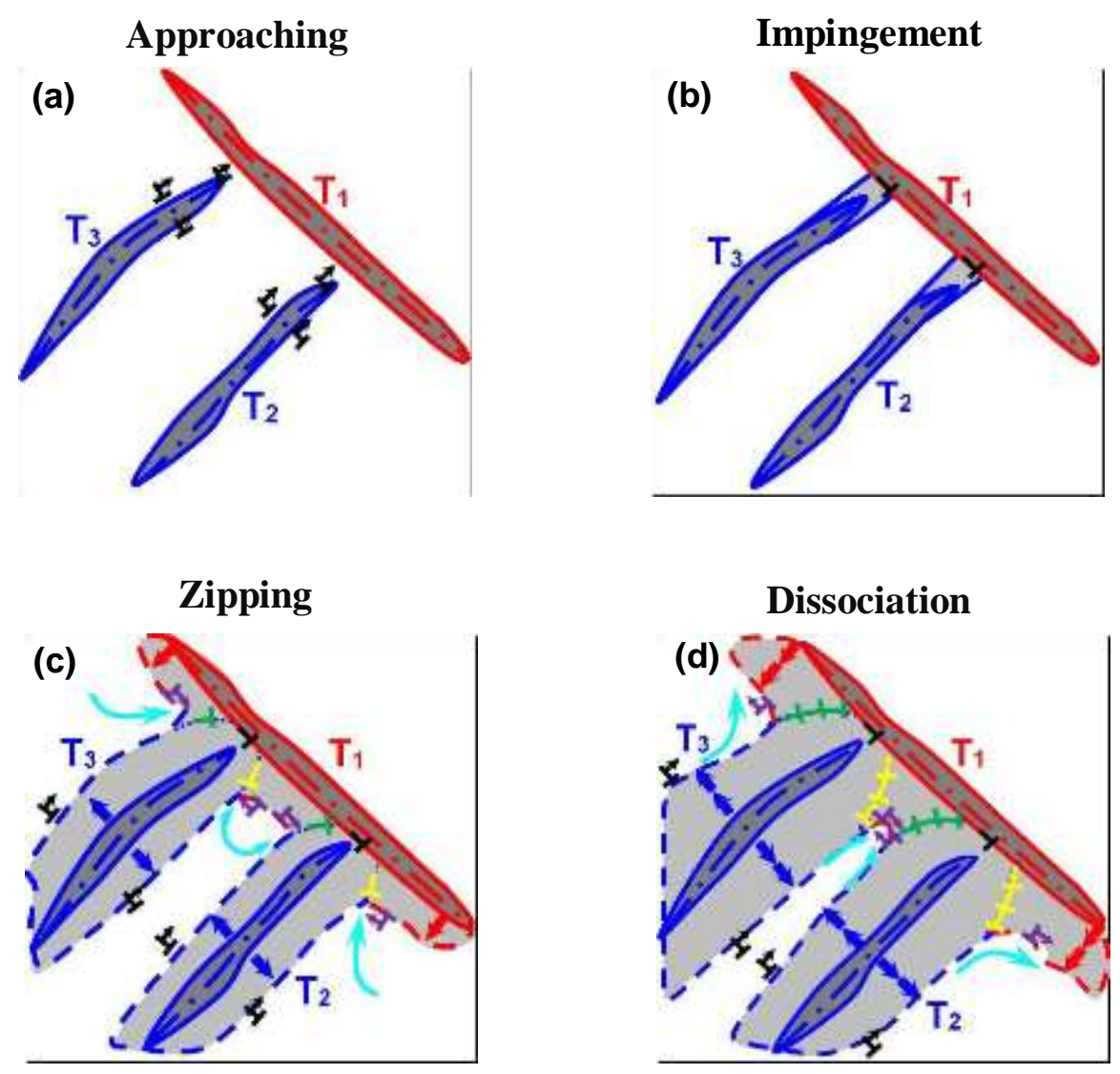

Fig. 4.19 Formation process of twin-twin boundaries: (a) two twins approaching the boundary of a pre-existing twin, (b) impingement of twinning dislocations on the twin boundary, (c) zipping process where dislocations between the twins in contact form common junctions, and (d) dissociation where twinning dislocations associated with one twin dissociate into twinning dislocations of the other twin.

The impingement process, shown in Fig.4.19(b), was encountered whenever the front tips of approaching twins (e.g., $\mathrm{T}_{2}$ and $\mathrm{T}_{3}$ ) were obstructed at the boundary of another pre-existent twin (e.g., $\mathrm{T}_{1}$ ). $\mathrm{T}_{2}$ and $\mathrm{T}_{3}$ were therefore responsible for the impingement of twinning dislocations on the twin boundary of $T_{1}$, leading to coincident twin-twin boundary planes (i.e., the common interface bisecting two twinning planes) with a similar character of the twinning dislocations to the incoming twins. Fig.4.19(c) and (d) are related to the stage at which $\left\{10 \overline{1}_{2}\right\}$ twins merged and continued to grow together. Two scenarios are possible based on the gliding mechanisms of 
twinning dislocations. A color code was used to indicate the dislocations in Fig.4.19(c) and (d), where twinning dislocations related to incoming twins $\mathrm{T}_{2}$ and $\mathrm{T}_{3}$ were colored in black and those for $\mathrm{T}_{1}$ (i.e., the pre-existing twin) in purple. The resultant junctions of twin-twin boundary dislocations were presented in yellow and green on each side of the contact surface, respectively. The configuration shown in Fig.4.19(c) was related to the zipping process defined as the formation of dislocation junctions piling up into a "dislocation wall", which corresponded to the twin-twin boundaries. Twinning dislocations related to the pre- existing twins (i.e., purple color in Fig.4.19(c)) were observed to be pushing inwards to accommodate the formation of the common junctions. The third option sketched in Fig.4.19(d) is related to the dissociation process which could be introduced as the formation of twin-twin boundaries upon dissociating the twinning dislocations of incoming twins (e.g., $\mathrm{T}_{2}$ and $\mathrm{T}_{3}$ ) into the twinning dislocations of the pre-existing twin (e.g., $\mathrm{T}_{1}$ ), with the formation of a residual. Yu et al. [102] reported that the residual formed after gliding of twinning dislocations towards the boundaries was left at the intersection of the two twins, corresponding to a junction. Hence, more resulting twin boundary dislocations were involved (i.e., dislocations presented in green and yellow in Fig.4.19(d)) compared with the previous zipping process, and the dislocations associated with the pre-existing twin (i.e., purple color in Fig.4.19(d)) were pointing outwards to accommodate the dissociation.

\subsubsection{Contribution of twinning to the deformation behavior}

The inadequate number of basal slip systems in $\mathrm{Mg}$ alloys made twinning substantial to accommodate plastic deformation, due to its lower CRSS compared to prismatic and pyramidal slip. Stepwise compression was performed along ED to facilitate extension twinning and its 
derivatives, which led to the observed texture formation in Fig.2 and the reorientation of the main texture components in Fig.4.2. Although narrow banded $\{10 \overline{1} 3\}-\{10 \overline{1} 2\}$ double twins could also accommodate compressive strains along the $c$-axis at RT [107], their limited growth prevented them from reaching the size and volume fraction of the $\{10 \overline{1} 2\}$ extension twins, and hence contributed much less to the shear accommodation. The $\{11 \overline{2} 1\}$ twins could not propagate as single embryonics as most of them grew to extremely thin and imperceptible lamellas. However, they played a major role in accelerating the propagation of $\{10 \overline{1} 2\}$ twins, by rapidly hosting them as secondary twins (Fig.4.17). Among the major impacts of twining on the deformation behavior of AZ31 is the tension-compression yield asymmetry, including a concave-shaped change in the flow curve and a rapid hardening regime [178].

Significant differences in twinning characteristics were observed above, depending on the activation of specific $\left\{10 \overline{1}_{2}\right\}$ twin variants. Also, twin-twin interactions between pre-existing and incoming twins belonging to different twin variants were identified. The foundation of the above-mentioned mechanisms could be understood by considering the following four main aspects: (1) the stresses for activating twinning and slip, (2) activities of twinning and slip during deformation, (3) the Hall-Petch effect by twinning-induced grain size change, and (4) twinninginduced change in activities of slip. The strain accommodated by twinning could be calculated as [109],

$$
\varepsilon_{T}=f_{T} \cdot \bar{m} \cdot \gamma_{T}
$$

where $\varepsilon_{T}$ represents the twinning strain, $f_{T}$ is the volume fraction of twins, $\bar{m}$ is the average Schmid factor of the twin variant, and $\gamma_{T}$ is the characteristic twinning shear. The total strain 
$\varepsilon_{\text {total }}$ could be expressed as the sum of contributions of the slip strain $\varepsilon_{\text {slip }}$ and the twinning strain $\varepsilon_{T}$, i.e.,

$$
\varepsilon_{\text {total }}=\varepsilon_{T}+\varepsilon_{\text {slip }} .
$$

Thus the fractional contribution of twinning during deformation (i.e., the increment in twinning strain per unit total strain) would be,

$$
\frac{d \varepsilon_{T}}{d \varepsilon_{\text {total }}}=\frac{d f_{T}}{d \varepsilon_{\text {total }}} \cdot \bar{m} \cdot \gamma_{T} .
$$

Similar to the present study, Hong et al. [110] plotted each of the contributions of twin and slip during compression along ED and observed that up to $\sim 4 \%$ compressive strain, twinning mostly dominated the deformation behavior. The capacity of twinning then started to decrease, and equal fractions of twinning and slip reached at $\sim 6.2 \%$. This could be linked to the present results, where beyond $\sim 6.0 \%$ compressive strain the twinning nucleation activity was observed to diminish considerably at the expense of the twin growth and twin boundary coalescence.

Since twin-twin boundary interactions have been of major interest in the current study, the effect of these twin boundaries was linked to the increase in the flow stress since they acted as obstacles against the dislocation motion [111]. Volume fraction of these boundaries could be considered inversely proportional to the effective grain size and their effect on the flow stress could be further linked to the Hall-Petch strengthening activated by the "twinning-induced" grain size change. The Hall-Petch hardening stress $\Delta \sigma_{H-P}$ could then be expressed as [110],

$$
\Delta \sigma_{H-P}=k\left(\frac{1}{\sqrt{d_{\text {eff }}}}-\frac{1}{\sqrt{d_{\text {initial }}}}\right),
$$


where $d_{\text {initial }}$ is the initial grain size, $d_{\text {eff }}$ is the effective grain size (at a given strain) and $k$ is the Hall-Petch coefficient. Hence, the various twinning mechanisms discussed in this study had the effect of reducing $d_{\text {eff }}$ through the stepwise compression and leading to a more significant HallPetch stress and supports the observed strain hardening mechanisms via twinning deformation.

\subsection{Summary}

The present work combines detailed observations on single and double twin nucleation, growth, and interaction in an extruded AZ31 Mg alloy. Major findings are:

1. The propagation of a single twin variant led to a relatively fast saturation of twin nucleation after which the increase in strain was predominantly accommodated by the growth of existing twin lamellas.

2. For double twin variants, the intersecting twins led to the confinement of the spaces constrained by the fine twin lamellas. Various double twinning structures acknowledged theoretically or through atomistic simulations were experimentally observed.

3. New twin-twin interaction scenarios were identified depending on the strain amounts and paths of incoming twins and their impingement on the pre-existing twins.

4. The formation of the branching-like structure involved at least three non-co-zone $\{10 \overline{1} 2\}$ extension twin variants and Schmid factor analysis was used to understand its formation with respect to the activation and growth of stress-favored twin variants.

5. EBSD analyses revealed the nucleation and growth of multiple $\{10 \overline{1} 2\}$ secondary extension twin variants in the primary $\{11 \overline{2} 1\}$ embryonic twin, giving rise to the faster vanishing of the primary $\{11 \overline{2} 1\}$ embryonic twin during deformation. 
6. The apparent crossing between a very thin $\{10 \overline{1} 1\}$ contraction twin and two merging $\{10 \overline{1} 2\}$ extension twins was analyzed by means of the angular deviation between the twinning planes of the extension and contraction twins. 


\section{CHAPTER 5}

\section{CYCLIC DEFORMATION OF A LOW RE-CONTAINING ZEK100 Mg ALLOY}

\section{$5.1 \quad$ Introduction}

Low-cost and low RE-containing Mg alloys are being considered for the lightweight automotive applications to reduce fuel consumption and emissions. Design of $\mathrm{Mg}$ components requires strain-controlled LCF behavior. Thus, this investigation was aimed to evaluate the cyclic deformation characteristics and LCF life of a low (0.2 wt.\%) Nd-containing ZEK100 alloy compared to a RE-free $\mathrm{Mg}$ alloy and a high RE-containing alloy.

\subsection{Microstructure and EBSD measurements}

A 3-D EBSD band contrast (BC) mapping is shown in Fig.5.1(b). Black dots observed (i.e., pointed out by the arrows) indicate the presence of a small fraction of randomly distributed second-phase precipitates that will be later investigated by XRD phase identification and EDS. A more keen investigation on grain distribution is shown in Fig.5.2 which represents the EBSD orientation map on the RD-TD plane. Most of the grains were seen to be equiaxed with a quasiuniform distribution. This was mainly related to the happening of dynamic recrystallization (DRX) throughout the rolling process which was followed by annealing. Martin et al. [112] also observed recrystallized grains with a relatively small size $(\sim 5 \mu \mathrm{m})$ in the as-received ZEK100. Based on the standard color key provided, majority of grains on the RD-TD plane were oriented with their $c$-axis perpendicular to RD (i.e., their basal planes were parallel to RD). This is 
common for the rolled and extruded Mg alloys [113]. Also, the EBSD orientation map was in agreement with that reported by Aslam et al. [114] and Martin et al. [112] for a similar ZEK100O alloy.

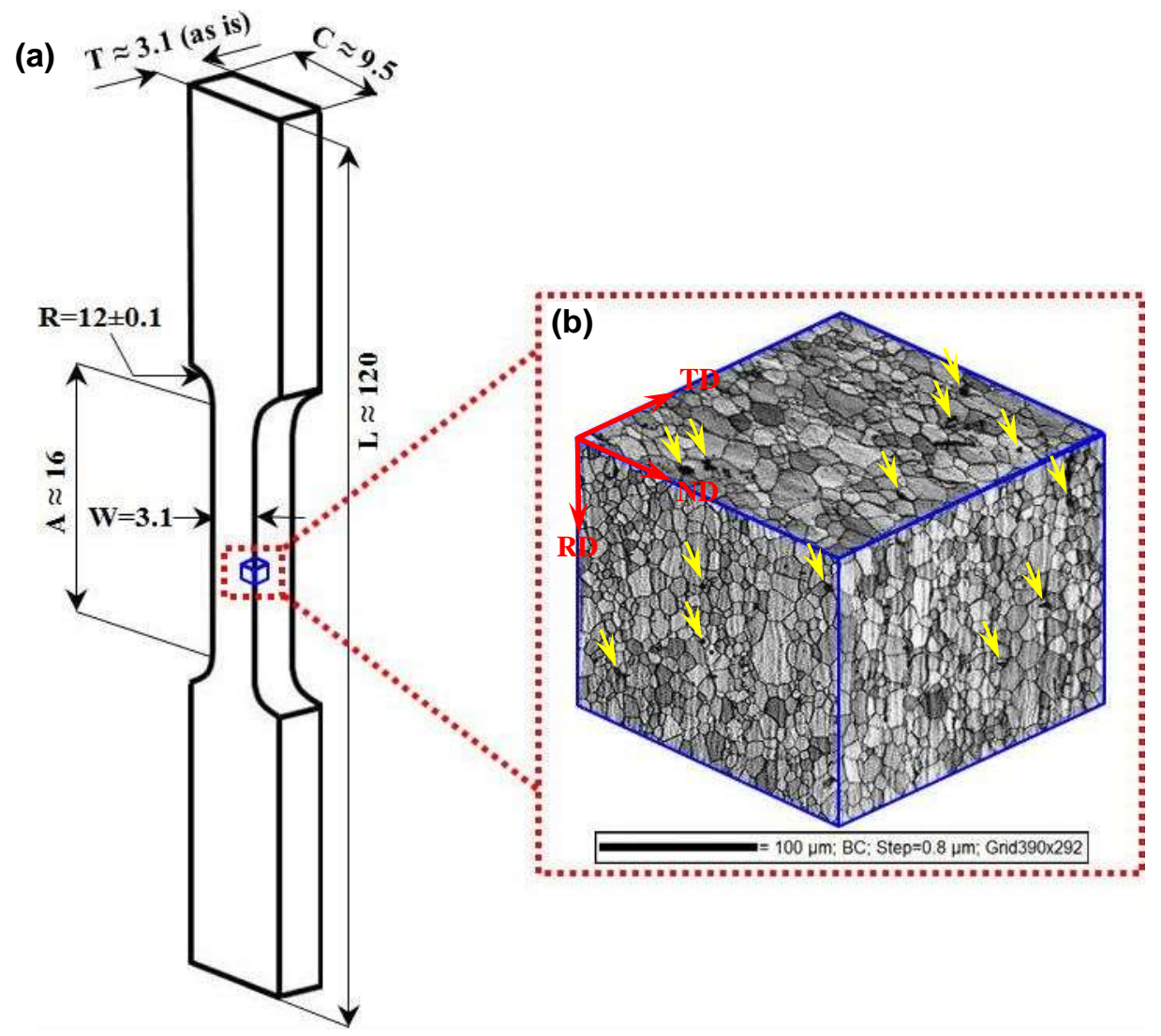

Fig.5.1 (a) Geometry and dimensions (in $\mathrm{mm}$ ) of fatigue test specimens, and (b) threedimensional EBSD band contrast (BC) mapping of the rolled ZEK100 Mg alloy in an annealed state, where RD stands for the rolling direction, TD denotes the transverse direction, and ND indicates the normal direction.

\subsection{X-ray diffraction analysis}

Fig.5.3 shows the XRD pattern of the ZEK100. In addition to the obviously dominant $\alpha-\mathrm{Mg}$ phase, both $\mathrm{Zn}$ and $\mathrm{Nd}$-containing intermetallic compounds were detected in the form of $\mathrm{MgZn}$ 
and $\mathrm{Mg}_{12} \mathrm{Nd}$ phases. $\mathrm{MgZn}$ and $\mathrm{Mg}_{12} \mathrm{Nd}$ could also be seen from a typical back-scattered electron SEM image as shown in Fig.5.4. The EDS point analysis revealed an atom percent of 91.6\% $\mathrm{Mg}$ and $8.4 \% \mathrm{Nd}$ for particle $\mathrm{A}$, which had an atomic ratio close to that of $\mathrm{Mg}_{12} \mathrm{Nd}$. Similarly, an atom percent of $56.2 \% \mathrm{Mg}$ and $43.8 \% \mathrm{Zn}$ was obtained for particle $\mathrm{B}$, which was close to the atomic ratio of $\mathrm{MgZn}$. Indeed, $\mathrm{Mg}_{12} \mathrm{Nd}$ and $\mathrm{MgZn}$ could be seen from the $\mathrm{Mg}-\mathrm{Nd}$ binary phase diagram [115] and the Mg-Zn binary phase diagram [116], respectively.
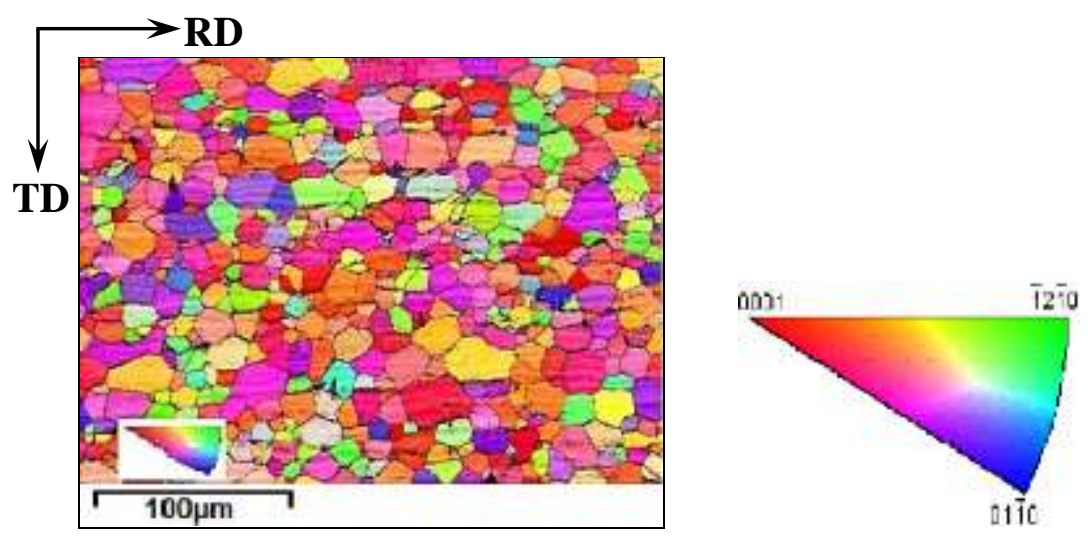

Fig.5.2 EBSD orientation map on the RD-TD plane of the rolled ZEK100 Mg alloy.

\subsection{Characterization of crystallographic texture}

Fig.5.5 displays (0001), (10 $\overline{1} 0)$ and (1011) pole figures of the ZEK100. The observed texture intensities were considerably lower compared to the RE-free AZ31 Mg alloy previously seen in Fig.4.2(a). Texture intensity of 3.7 MRD was observed for ZEK100 for the (0001) basal plane, where the $c$-axes of most grains were aligned perpendicular to the RD. Similarly, texture investigations were conducted for ZEK100 by Min and Lin [78], who reported a maximum intensity of 3.621 MRD for the (0001) pole figure, nearly the same as the present value. 


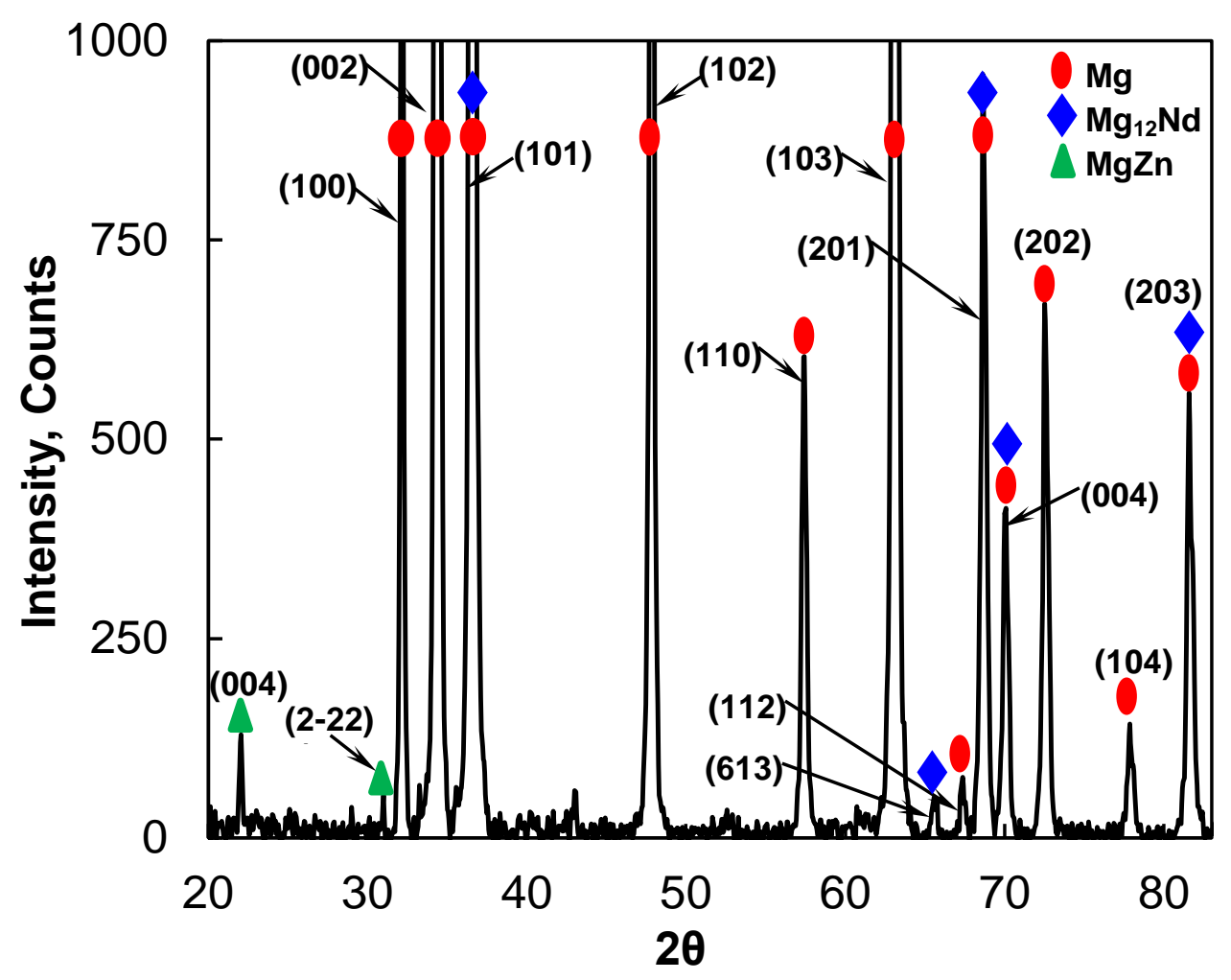

Fig.5.3 X-ray diffraction pattern of the ZEK100 Mg alloy.

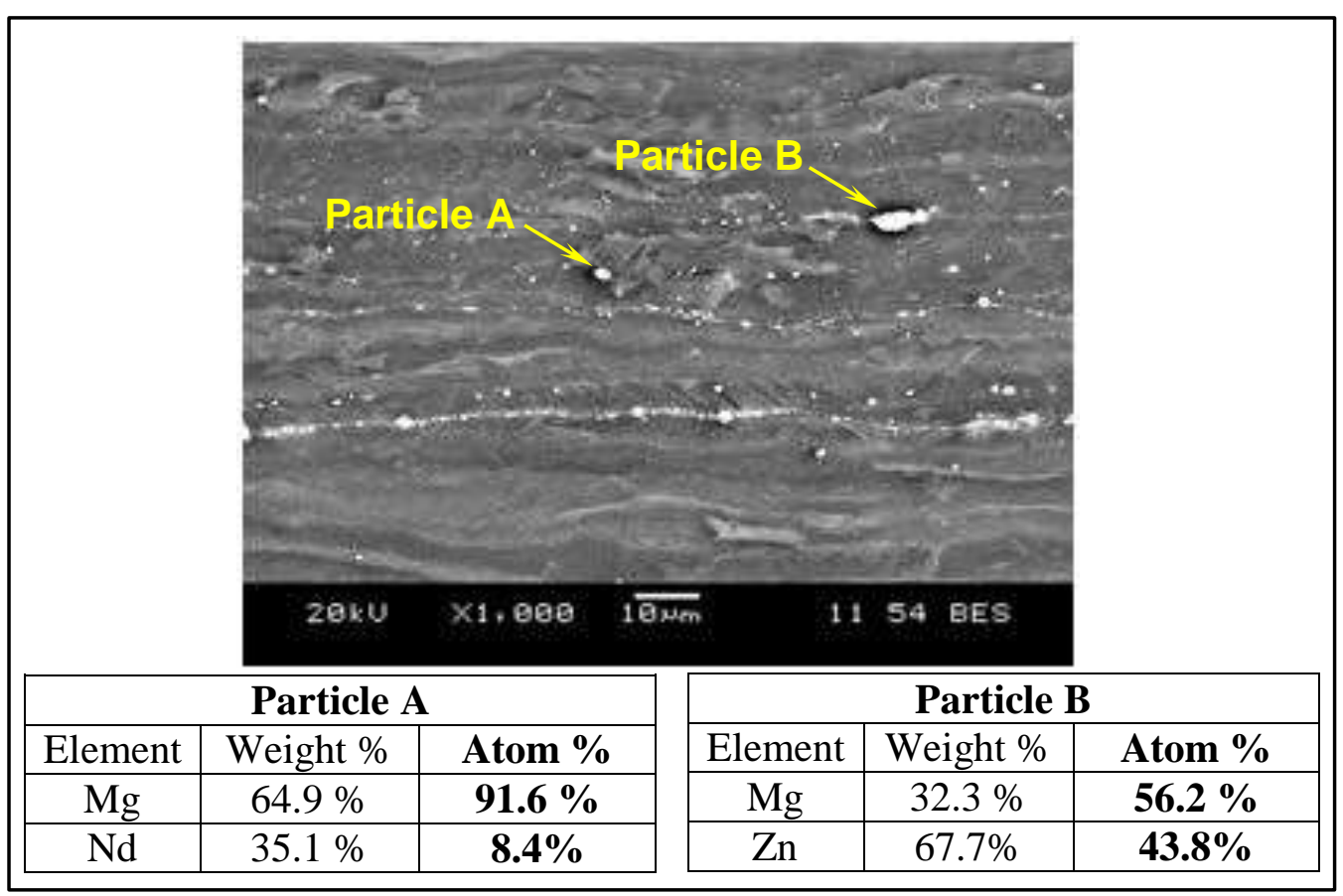

Fig.5.4 SEM back-scattered electron image specifying EDS point analysis locations and the corresponding composition at particles $\mathrm{A}$ and $\mathrm{B}$, respectively. 
The texture exhibited a TD-split basal texture and was attributed to typical $\mathrm{Mg}-\mathrm{X}-\mathrm{RE}$ ternary alloys [117]. Compared with pure Mg or other commercial Mg alloys, it was clear that the addition of a small amount of $\mathrm{Nd}$ led to texture weakening. One reason for texture weakening was likely to be associated with the formation of solid solution of RE elements in $\mathrm{Mg}$, which increased the difficulty for the rotation of basal planes (or twinning) due to the difference in the atomic radii between $\mathrm{Mg}$ and $\mathrm{RE}$ elements. Since the atomic size of all $\mathrm{RE}$ elements is significantly larger than that of $\mathrm{Mg}$, a substitutional $\mathrm{RE}$ atom would cause an intense lattice distortion, i.e., impose compressive strains in its vicinity [118]. Another reason for texture weakening was the presence of second-phase precipitates $\mathrm{Mg}_{12} \mathrm{Nd}$ and $\mathrm{MgZn}$. As reported by Humphreys [119], precipitation of second-phase particles could delay the dislocation movement and form stress concentrations adjacent to the precipitates which would enhance the particle simulated nucleation (PSN) of DRX and thus result in the lessening of basal texture.

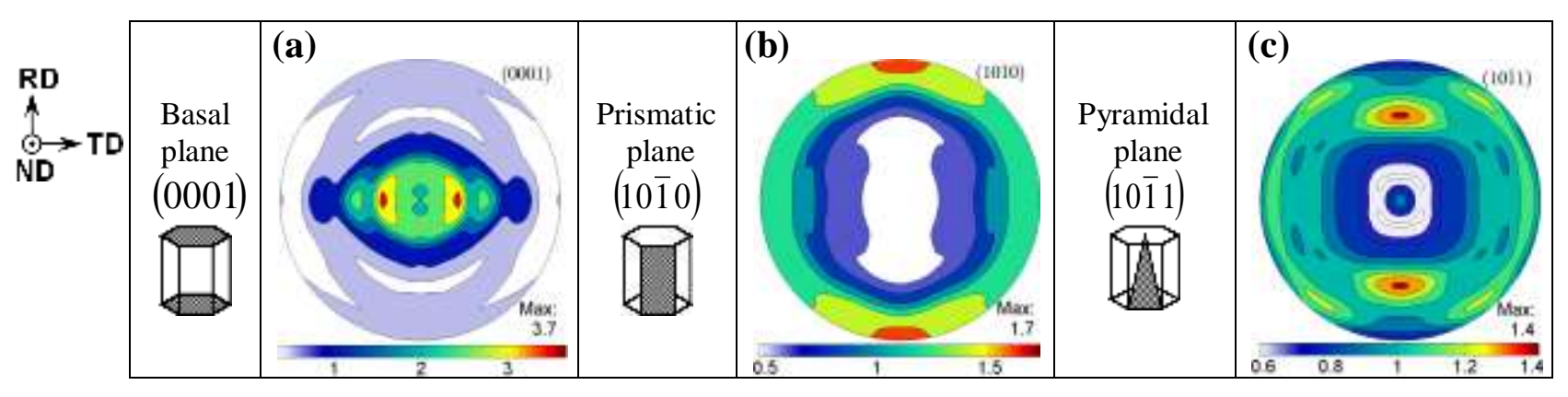

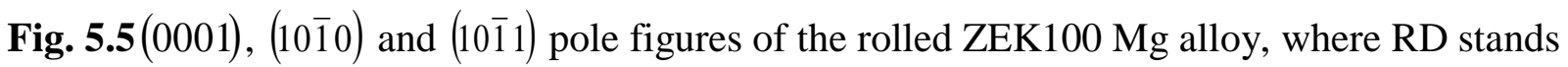
for the rolling direction, TD for the transverse direction, and ND for the normal direction.

\subsection{Hysteresis loops and tensile properties}

Stress-strain hysteresis loops of the first, second and mid-life cycles obtained at a total strain amplitude of $1.2 \%$ and a strain ratio of $R_{\mathrm{S}}=-1$ for the ZEK100 alloy are presented in Fig.5.6, 
where GW103K [75] and AM30 [120] were added for the sake of comparison. ZEK100 displayed a tensile yield strength (TYS) of $225 \mathrm{MPa}$ which lay in-between the two values obtained for AM30 and GW103K (i.e., 200 MPa [120] and 240 MPa [75], respectively). This was expected, since ZEK100 (with 0.2 wt.\% Nd) was compared on purpose to a RE-free Mg alloy (AM30) and a high RE-containing Mg alloy (GW103K with 10 wt.\% Gd and 3 wt.\% Y). This comparison once again brought up one of the major benefits from the addition of RE elements to Mg alloys even with moderate amounts, such the case of ZEK100.

The hysteresis loops of ZEK100 in Fig.5.6 fell in-between those of the almost symmetrical GW103K and the tilted AM30, especially in the compressive phase. Due to the presence of a high amount of RE elements, GW103K displayed a practically symmetrical hysteresis loop, similar to that in the fcc metals, which were controlled by the dislocation slip rather than twinning deformation [47].

\subsection{Cyclic stress and strain responses}

Evolution of stress amplitudes and plastic strain amplitudes with respect to the number of cycles at different strain amplitudes from $0.2 \%$ to $1.2 \%$ is shown in Fig.5.7and 5.8, respectively, under a semi-logarithmic scale along the $\mathrm{X}$ axis. Both stress amplitudes and plastic strain amplitudes augmented, whereas fatigue life of the material diminished with increasing total strain amplitudes. In addition, a slight cyclic softening could be seen at two highest strain amplitudes namely $1.0 \%$ and $1.2 \%$, however, almost cyclic stabilization was noted for the remaining strain amplitudes especially at lower values of $0.2 \%-0.4 \%$. 

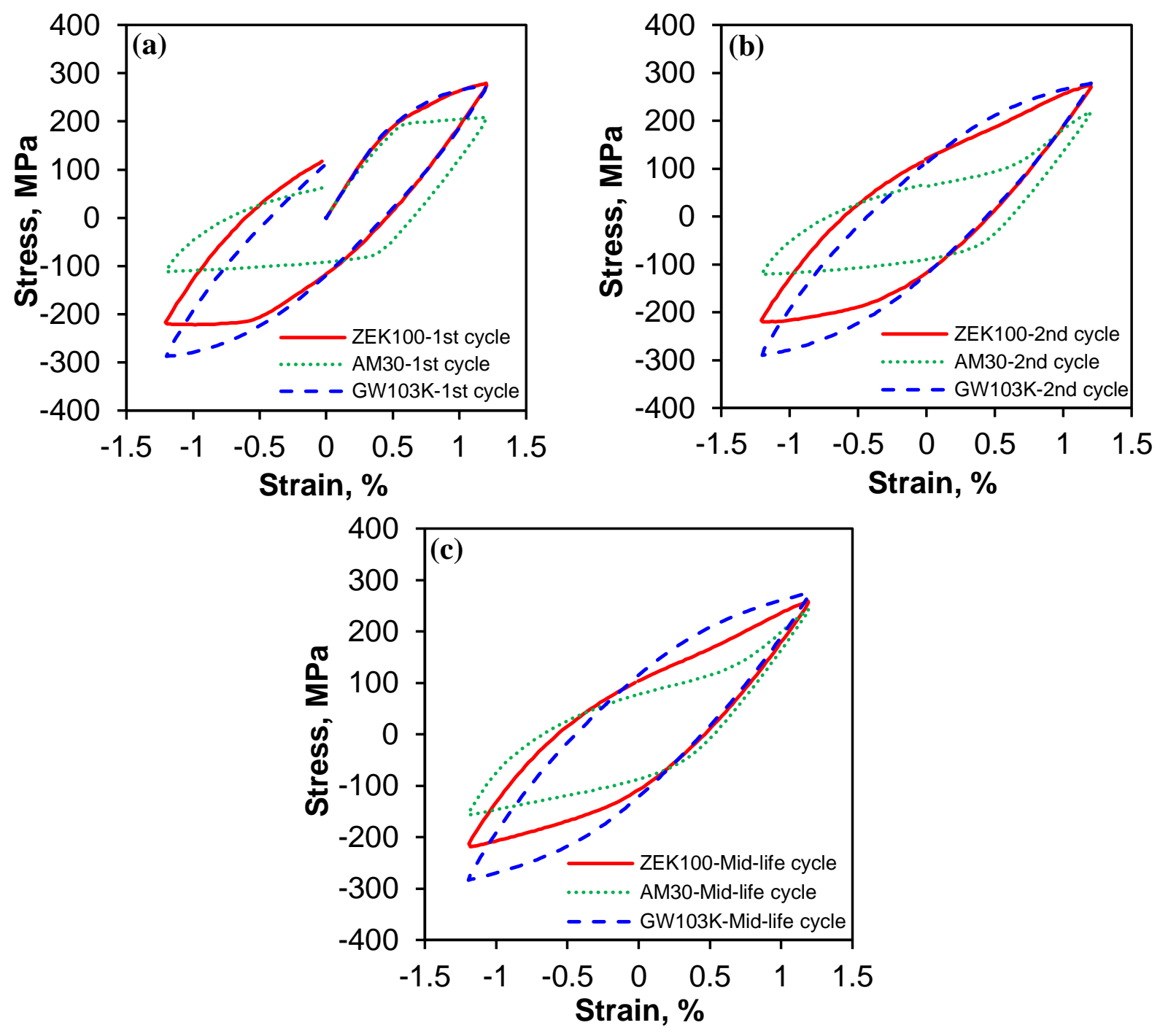

Fig.5.6 Characteristic stress-strain hysteresis loops of the (a) first cycle, (b) second cycle, and (c) mid-life cycle at a total strain amplitude of $1.2 \%$ and strain ratio $R_{\mathrm{S}}=-1$ for the rolled ZEK100 alloy, in comparison with the extruded AM30 and GW103K alloys [75,120].

Unlike the present ZEK100, cyclic hardening occurred at higher strain amplitudes due to the occurrence of extensive twinning in the descending and compressive phase and detwinning in the ascending and tensile phase during cyclic deformation along the extrusion direction in the REfree wrought Mg alloys (e.g., AM30 and AZ31) [120,121]. In the case of high RE-containing 
alloys such GW103K with about 10 wt.\% Gd and 3 wt.\% Y [75], cyclic stabilization was observed up to a strain amplitude of $\sim 1.0 \%$.

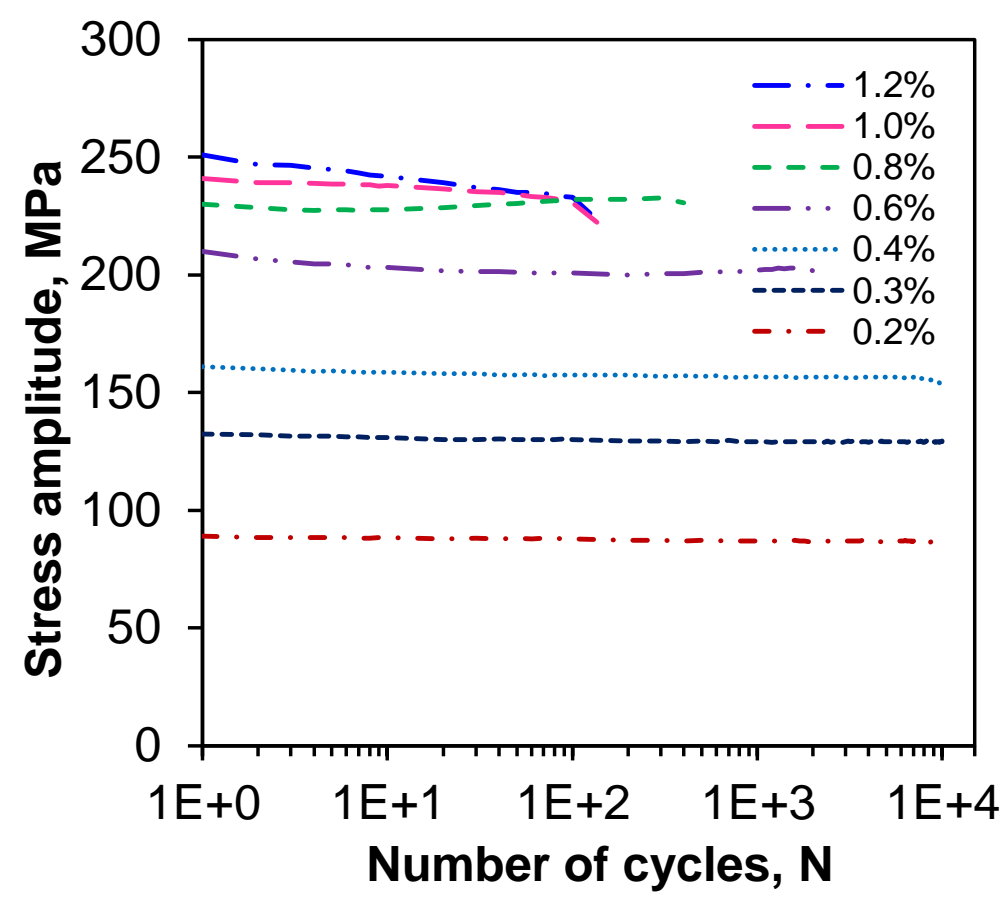

Fig.5.7 Stress amplitude vs. the number of cycles at different total strain amplitudes.

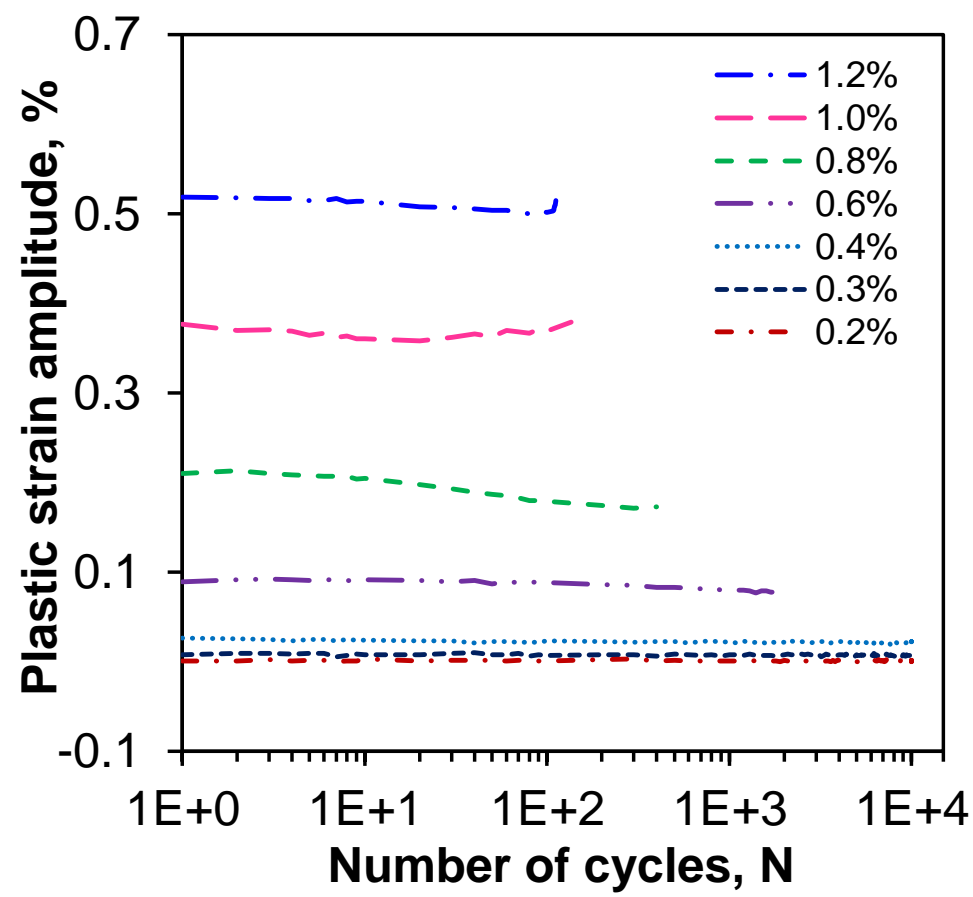

Fig.5.8 Plastic strain amplitude vs. the number of cycles at different total strain amplitudes. 


\subsection{Fatigue life and strain-life fatigue parameters}

Fig.5.9 displays the total strain amplitude $\Delta \varepsilon_{\mathrm{t}} / 2$ as a function of the number of cycles to failure (i.e., fatigue life) for ZEK100, in comparison with the data reported in the literature for both REfree and RE-containing wrought $\mathrm{Mg}$ alloys $[75,77,120,122,123]$. The run-out data points where no failure occurred at $10^{7}$ cycles or more are labeled by horizontally directing arrows.

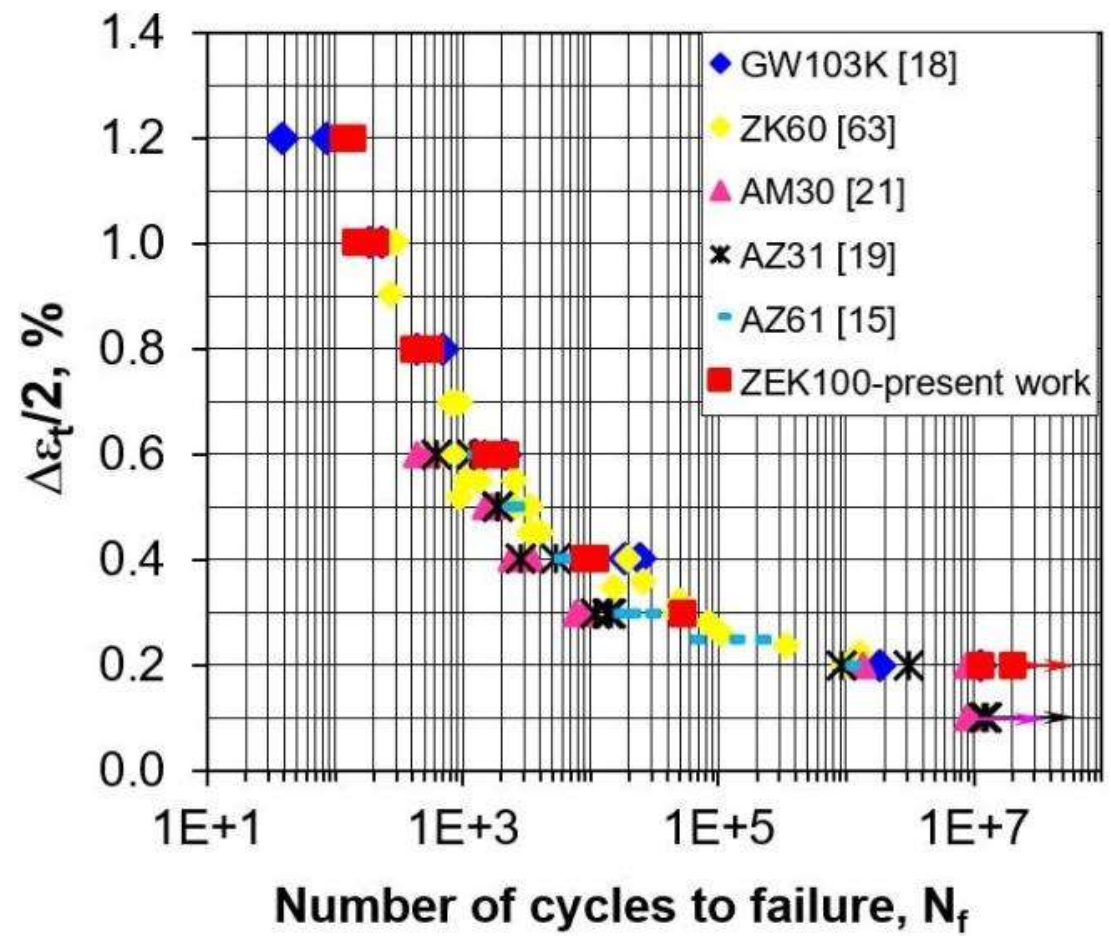

Fig.5.9 Total strain amplitude as a function of the number of cycles to failure for the rolled ZEK100 Mg alloy, in comparison with the data reported in the literature for various wrought $\mathrm{Mg}$ alloys.

A relatively enhanced fatigue life was obtained for ZEK100, when compared to the RE-free alloys (AM30, AZ31 and AZ61). Compared to the rest of the RE-containing alloys (GW103K and ZK60), ZEK100 showed the ability of keeping up for a high and equivalent number of 
cycles to failure at the most total strain amplitudes, although it is an alloy that contained the least RE element ( 0.2 wt.\% Nd) among the three alloys. This suggests the effectiveness of $\mathrm{Nd}$ addition along with the proper processing or alloy state (annealing O-state), which led to a fairly good strength and ductility ( $\sigma_{U T S} \sim 300 \mathrm{MPa}$, and elongation $\sim 13.3 \%$ at RT, and $1 \times 10^{-2} \mathrm{~s}^{-1}$ ), since strain-controlled fatigue life is related to both characteristics. Fatigue behavior could also be expressed through the total strain amplitude consisting of the elastic and plastic strain amplitudes separately, where the elastic strain component was denoted by the Basquin's equation and the plastic strain constituent was known as the Coffin-Manson relation. The equation can be expressed as $[77,120,124,125]$,

$$
\frac{\Delta \varepsilon_{t}}{2}=\frac{\Delta \varepsilon_{e}}{2}+\frac{\Delta \varepsilon_{p}}{2}=\frac{\sigma_{f}^{\prime}\left(2 N_{f}\right)^{b}}{E}+\varepsilon_{f}^{\prime}\left(2 N_{f}\right)^{c},
$$

where $E$ is the Young's modulus (during fatigue testing, the average value for ZEK100 was $\sim 44.9 \mathrm{GPa}), N_{f}$ is the number of cycles to failure, $\sigma_{f}^{\prime}$ is the fatigue strength coefficient, $b$ is the fatigue strength exponent, $\varepsilon_{f}^{\prime}$ is the fatigue ductility coefficient, and $c$ is the fatigue ductility exponent. Fig.5.10 illustrates the elastic, plastic, and total strain amplitudes as a function of the number of reversals to failure $\left(2 N_{f}\right)$.

In order to make sure that cyclic stabilization, also called cyclic saturation, has already occurred, the stress and strain values of the mid-life cycles were used. Hence, the fatigue life parameters obtained by means of Equ.(5.1) were presented in Table 5.1. 


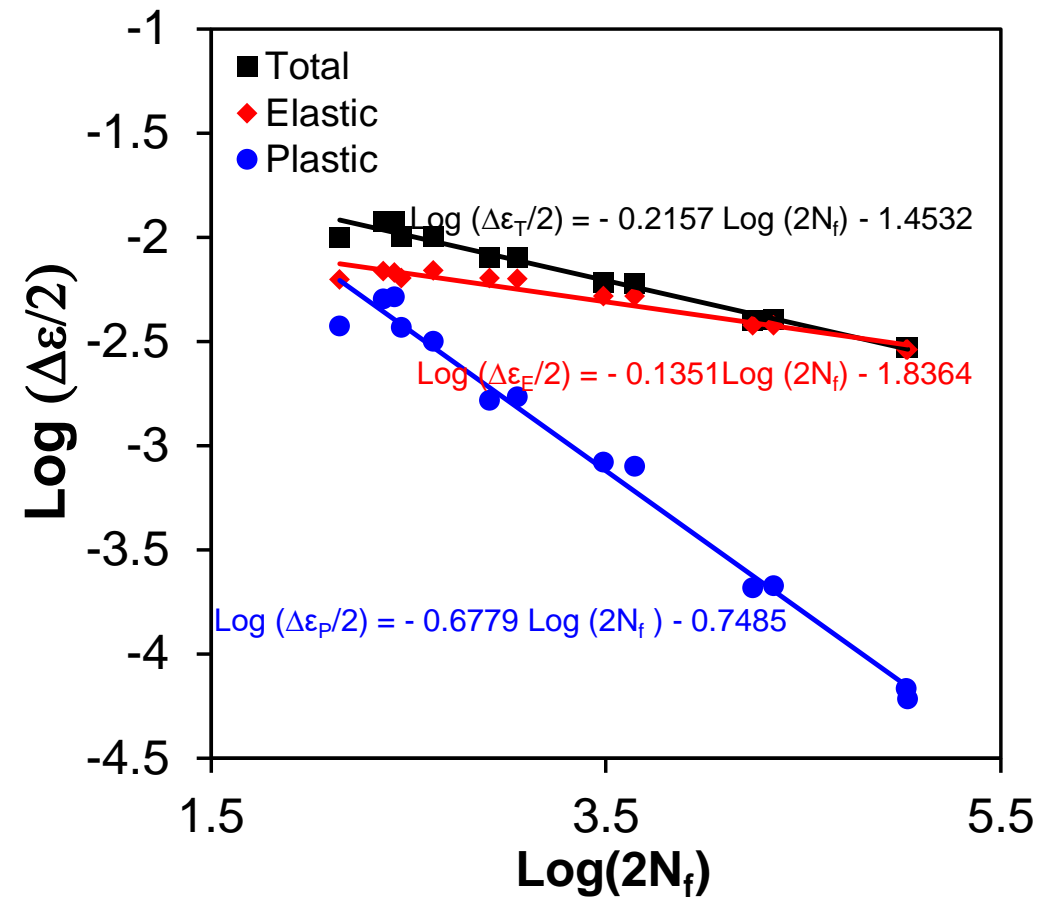

Fig.5.10 Evaluation of strain-life fatigue parameters in the form of log-elastic, plastic and total strain amplitudes vs. log-number of reversals to failure, respectively.

Table 5.1 Low cycle fatigue parameters obtained for the rolled ZEK100 Mg alloy.

\begin{tabular}{lc}
\hline Low cycle fatigue parameters & ZEK100 \\
\hline Cyclic strain hardening exponent, $n^{\prime}$ & 0.15 \\
Cyclic strength coefficient, $K^{\prime}, \mathrm{MPa}$ & 542 \\
Fatigue strength coefficient, $\sigma_{f}^{\prime}, \mathrm{MPa}$ & 654 \\
Fatigue strength exponent, $b$ & -0.14 \\
Fatigue ductility coefficient, $\varepsilon_{f}^{\prime}$ & 0.18 \\
Fatigue ductility exponent, $c$ & -0.68 \\
\hline
\end{tabular}

\section{8 $\quad$ Fractography}

Fig.5.11(a) and (b) display an overall view of fatigued samples tested at total strain amplitudes of $0.4 \%$ and $1.0 \%$, respectively. 

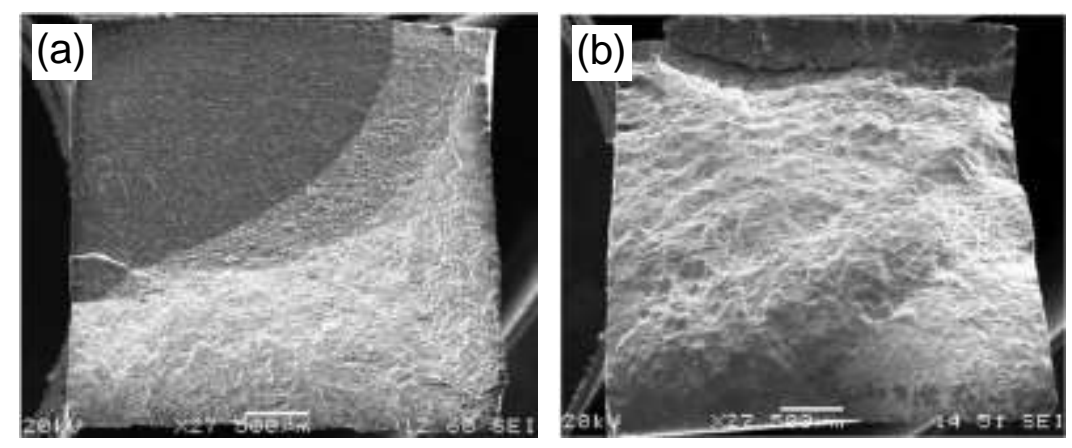

Fig.5.11 SEM micrographs of overall fracture surfaces of the specimens fatigued at a strain amplitude of (a) $0.4 \%$ and (b) $1.0 \%$, respectively.

It is seen that fatigue cracks initiated from the specimen surface and crack initiation occurred from the specimen surface or near surface defects. It was clear that fatigue crack propagation area was larger at lower strain amplitude of $0.4 \%$ than at higher strain amplitude of $1.0 \%$. SEM images taken at a higher magnification in the crack propagation area (Fig.5.12(a) and (b)) showed that at both strain amplitudes, the fatigue crack propagation region encompassed some striation-like features which were basically the distinct line markings appearing on the fractured surface, and were noted to be perpendicular to the crack propagation direction.
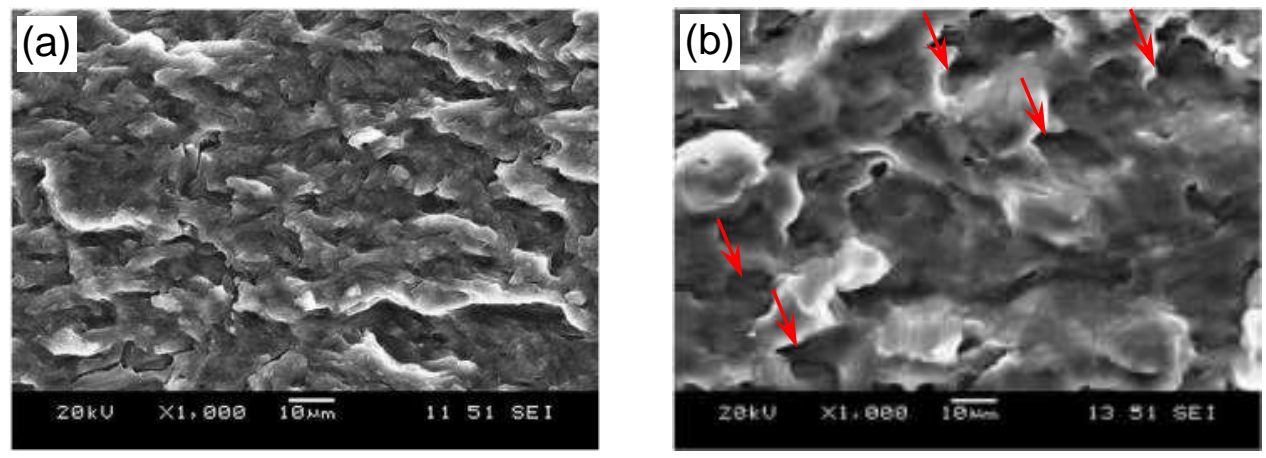

Fig.5.12 SEM micrographs of the fatigue crack propagation area in the specimens fatigued at strain amplitude of (a) $0.4 \%$ and (b) $1.0 \%$, respectively. 
Few secondary cracks could also be perceived. Some shallow dimples along with tear ridges marked by arrows were visible, indicating the presence of larger plastic deformation in front of the crack tip at the higher strain amplitude, considered in this case as a typical behavior.

\subsection{Twinning-detwinning behavior}

As previously mentioned, wrought $\mathrm{Mg}$ alloys are deformed such that majority of grains have their basal planes approximately parallel to the process direction. Therefore, if a load is applied along this direction, the activation stress would be comparatively low for the deformation twinning in compression (i.e., extension twinning) or in tension (i.e., basal slip and/or detwinning) [75]. The activation stress would be fairly high in the case of prismatic and pyramidal slip systems for Mg alloys at room temperature [126]. Consequently, twinning and de-twinning interchange by alternating activities at the time of cyclic loading. During cyclic deformation residual twins got progressively cumulated with increasing number of cycles.

Fig.5.13 displays the optical micrographs of the areas near the fracture surface at strain amplitude of $1.2 \%$, showing the overall distribution of residual twins in a ZEK100 fatigued sample. Although winning was the dominating deformation mechanism in ZEK100, a lesser extent of twinning was observed in Fig.5.13(c). This implicates that in some regions basal slip was dominant, leading to the better symmetry of hysteresis loops of ZEK100 in comparison with RE-free Mg alloys. Twinning was highly implicated in causing the asymmetry of wrought $\mathrm{Mg}$

alloys, generally characterized by tension-compression asymmetry as demonstrated by Wu et al. 
[117]. This asymmetry was mainly accredited to the extensive $\{10 \overline{1} 2\}\{10 \overline{1} 1\rangle$ extension twinning under compressive deformation along the process direction [125].

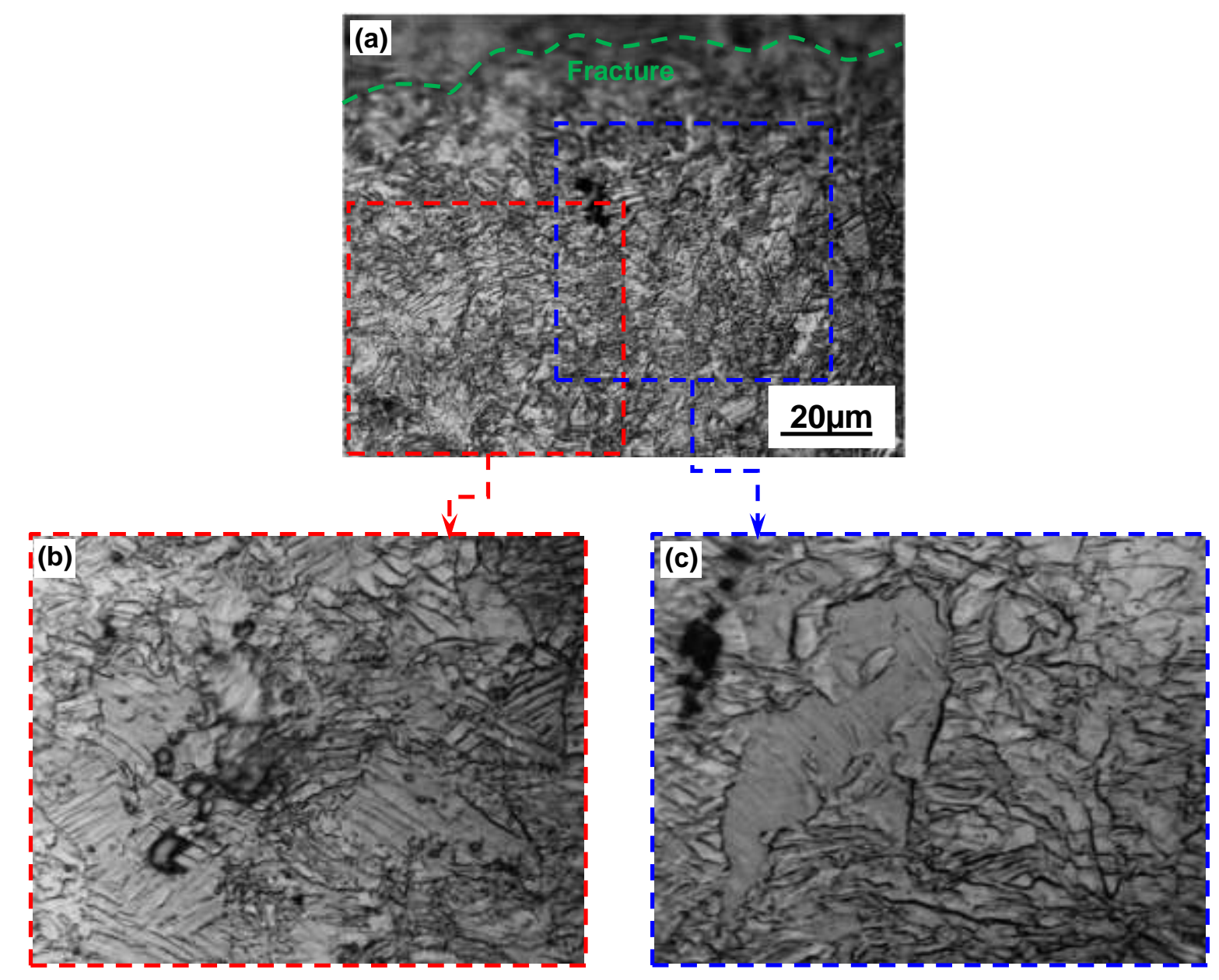

Fig.5.13 (a) Optical micrographs of the areas near the fracture surface, showing the overall distribution of residual twins in a sample of ZEK100 alloy fatigued at a strain amplitude of 1.2\% with emphasis on two regions (b) and (c), respectively.

\section{$5.10 \quad$ Summary}

This investigation helped draw the following conclusions: 
1. Microstructure of ZEK100 in the annealing state consisted of equiaxed grains due to the occurrence of DRX. The alloy contained some $\mathrm{Mg}_{12} \mathrm{Nd}$ and $\mathrm{MgZn}$ particles. The addition of 0.2 wt.\% $\mathrm{Nd}$ along with $1.3 \mathrm{wt} . \% \mathrm{Zn}$ and $0.25 \mathrm{wt} . \% \mathrm{Zr}$ led to the weakening of basal texture in comparison with the extruded RE-free AZ31 and AM30 alloys.

2. While slight cyclic softening was observed at high strain amplitudes, cyclic stabilization occurred at lower strain amplitudes. Fatigue life of ZEK100 determined via the straincontrolled fatigue tests was longer than that of the extruded RE-free Mg alloys, as a result of a fairly good combination of strength with ductility in this alloy.

3. The extent of the asymmetry of the ZEK100 loops was effectively improved in comparison with the extruded RE-free AZ31 and AM30 alloys. This indicated that the small addition of 0.2 wt.\% Nd in the ZEK100 alloy played a significant role in overpowering the incidence of excessive twinning via texture weakening and grain refinement.

4. Fatigue crack initiation was observed to occur from the specimen surface or near-surface imperfections. Crack propagation region encompassed fatigue striation-like features, which were normal to the crack propagation direction. 


\section{CHAPTER 6}

\section{ANELASTIC BEHAVIOR AND EFFECT OF STRAIN RATIO ON CYCLIC DEFORMATION OF ZEK100 Mg ALLOY}

\subsection{Introduction}

The purpose of this study was to identify the effect of strain ratio on cyclic deformation behavior of a rolled ZEK100-O Mg alloy. The anelastic behavior of this alloy largely remained arising from the twinning and detwinning, with the strain ratio identified as an influential parameter via sensitivity analyses. The anelastic strain amplitude, along with three newly-defined parameters (eccentricity, angle deviation, and relative slope change) all decreased with increasing strain ratio, reflecting more symmetric hysteresis loops.

\subsection{Evolution of fatigue behavior with strain ratio}

Stress-strain hysteresis loops of first, second and mid-life cycles for ZEK100 fatigued at different strain ratios $R_{\varepsilon}$ are shown in Fig.6.1. The observation of Fig.6.1(a) allows concluding that the initial tensile phase of the first cycle followed a sort of harmonization through the same path, referred to as Masing behavior as documented by Christ and Mughrabi [127]. The strain ratio was increased from $-\infty$ to +0.5 , where the peak tensile stress $\sigma_{\max }$ reached $\sim 300 \mathrm{MPa}$ (beyond the yield strength of $\sim 225 \mathrm{MPa}$ ) and thus a significant plastic deformation in the tensile phase was noted as seen from Fig.6.1(a), especially for $R_{\varepsilon}=0$ and 0.5. In essence, such a large plastic deformation in the tensile phase was attributed to the stipulated maximum strain $\varepsilon_{\max }$ of $3.2 \%$ for 
$R_{\varepsilon}=0.5$ and $1.6 \%$ for $R_{\varepsilon}=0$, as previously seen in Table 3.4 , which were much higher than the usually-used $0.2 \%$ strain offset for evaluating the YS. Similarly, from Fig.6.1(b) and (c), a noticeable shift of the hysteresis loops at $R_{\varepsilon}=0$ and 0.5 was observed and linked to the stipulation of the maximum and minimum strain limits (Table 3.4). It is clear that the asymmetry of the hysteresis loops were present initially at the first and second cycles, and became comparatively more symmetric in tension and compression at the mid-life cycles.
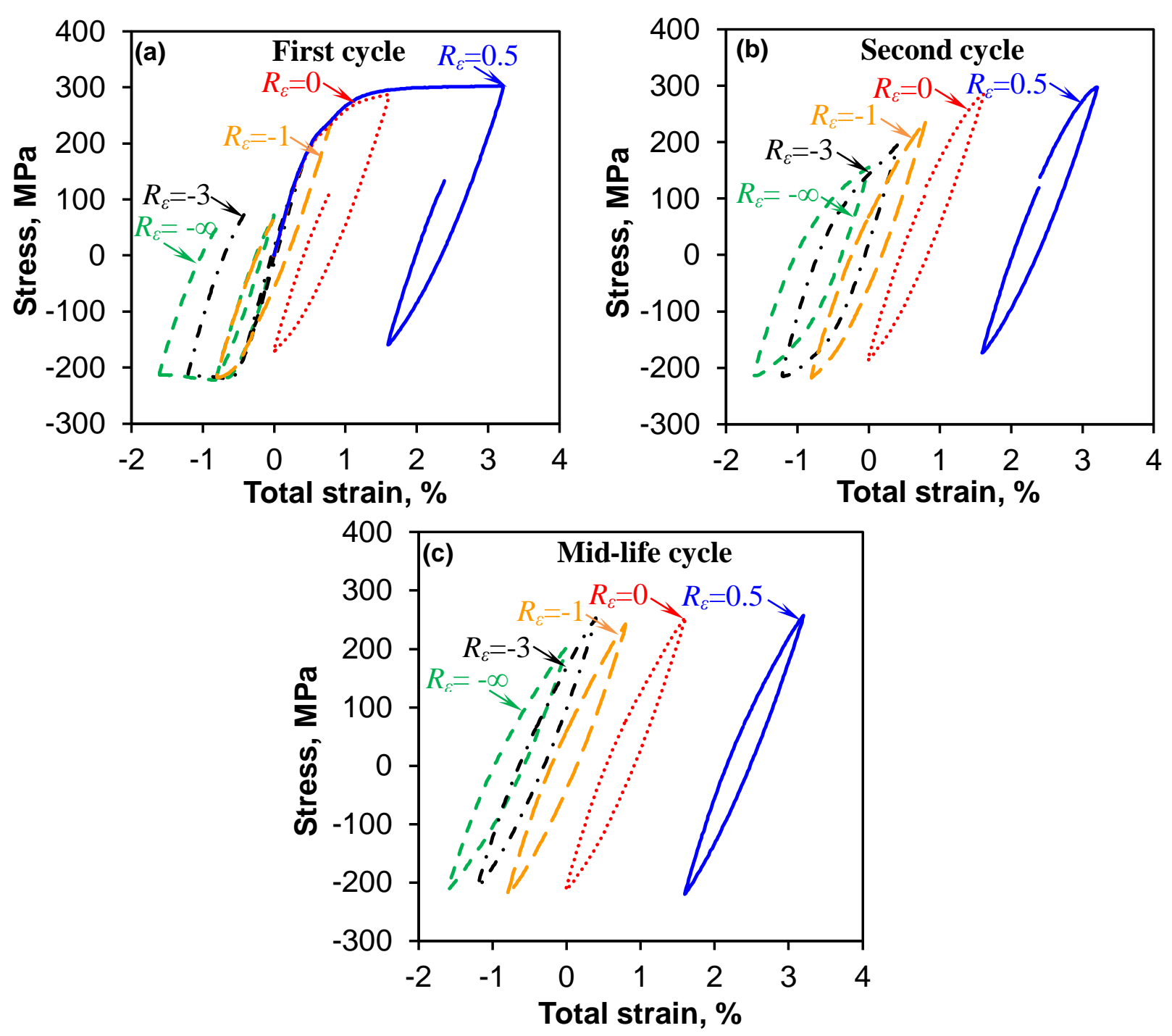

Fig.6.1 Stress-strain hysteresis loops of the rolled ZEK100 Mg alloy tested at different strain ratios at a total strain amplitude of $0.8 \%$, (a) first cycle, (b) second cycle, and (c) mid-life cycle. 
Evolution of stress amplitudes versus the number of cycles at different applied strain ratios on a semi-log scale is presented in Fig.6.2(a). A nearly cyclic stabilization persisted up to failure at strain ratios from -1 to +0.5 . A rise in the stress amplitude from the first to the second cycle at the strain ratios of $R_{\varepsilon}=-\infty$ and -3 was observed, where the rate of change for $R_{\varepsilon}=-\infty$ was more noticeable as highlighted in Fig.6.2(a). This was expected since a higher compressive stress was observed at $R_{\varepsilon}=-\infty$. Different features were reported in [77] for the RE-free AZ31, where the material exhibited high cyclic hardening at negative strain ratios which persisted but tended to weaken at positive strain ratios. Fig.6.2(b) displays the change of the plastic strain amplitude $\left(\Delta \varepsilon_{p} / 2\right)$ at different strain ratios during cyclic deformation. A decrease from the first to the second cycles was observed at all strain ratios, except for the case of $R_{\varepsilon}=-1$ and 0 values, where it maintained a nearly constant evolution up to failure.
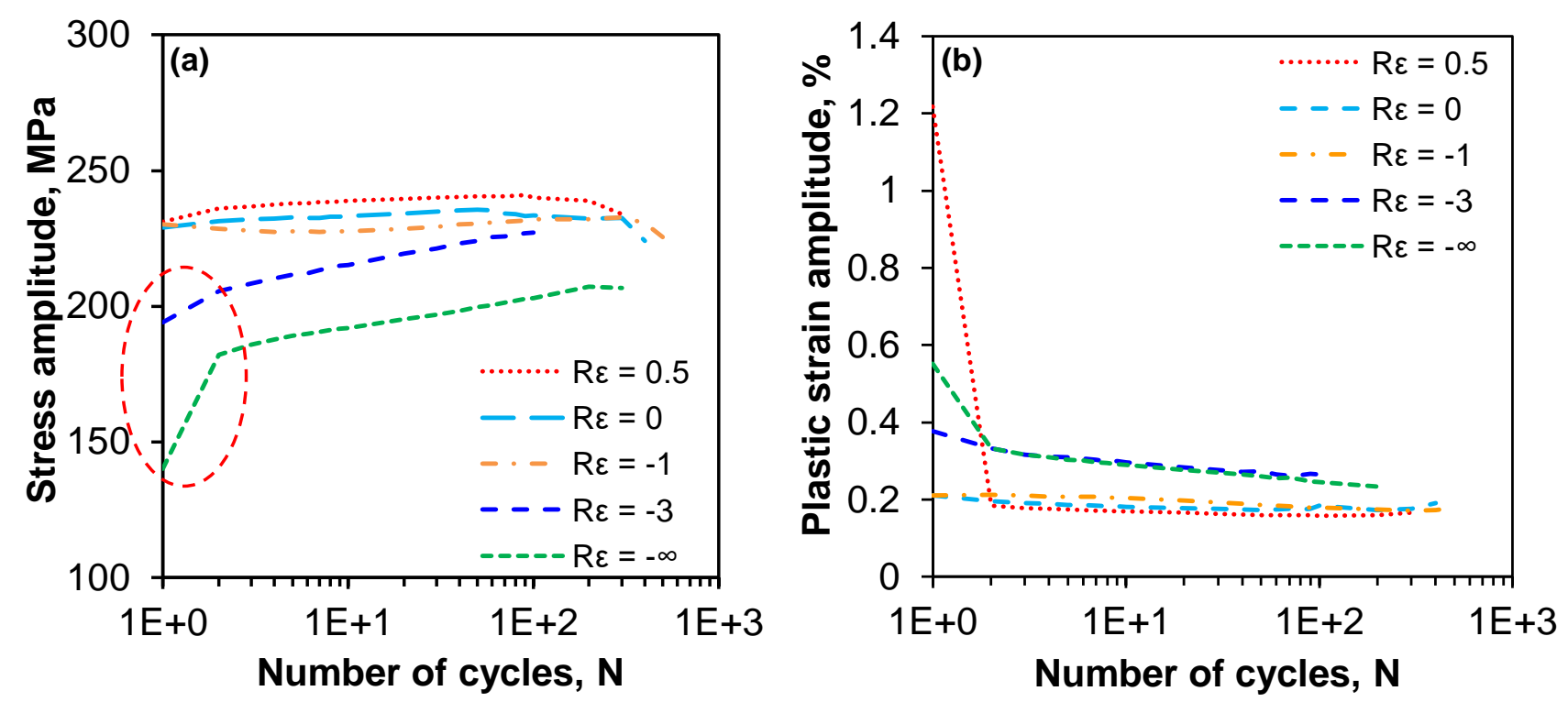

Fig. 6.2 (a) Stress amplitude and (b) plastic strain amplitude vs. the number of cycles at different strain ratios, at a total strain amplitude of $0.8 \%$. 
An abrupt drop was observed at $R_{\varepsilon}=0.5$ where a high initial $\Delta \varepsilon_{p} / 2$ of $\sim 1.2 \%$ was recorded, which exceeded the specified total strain amplitude of $0.8 \%$. Plastic strain amplitude decreased with increasing strain ratio or mean strain at $R_{\varepsilon}=-\infty$ and -3 , while it remained nearly constant from $R_{\varepsilon}=-1$ to 0.5 (Fig.6.2(b)). Similar results were obtained for the high RE-containing Mg alloy GW103K [75], the RE-free extruded AZ31 Mg alloy [77] and the semi-solid processed AZ91D [128]. The fatigue life or number of cycles to failure $N_{f}$ as a function of strain ratio is displayed in Fig.6.3 It was observed to increase with increasing strain ratio from $R_{\varepsilon}=-\infty$ to -1 , reach the maximum at $R_{\varepsilon}=-1$, and then decrease from $R_{\varepsilon}=-1$ to 0.5 . This was a result of the coupled role of cyclic stress amplitude and plastic strain amplitude which were inversely affected by the strain ratio, as previously seen from Fig.6.2(a) and (b).

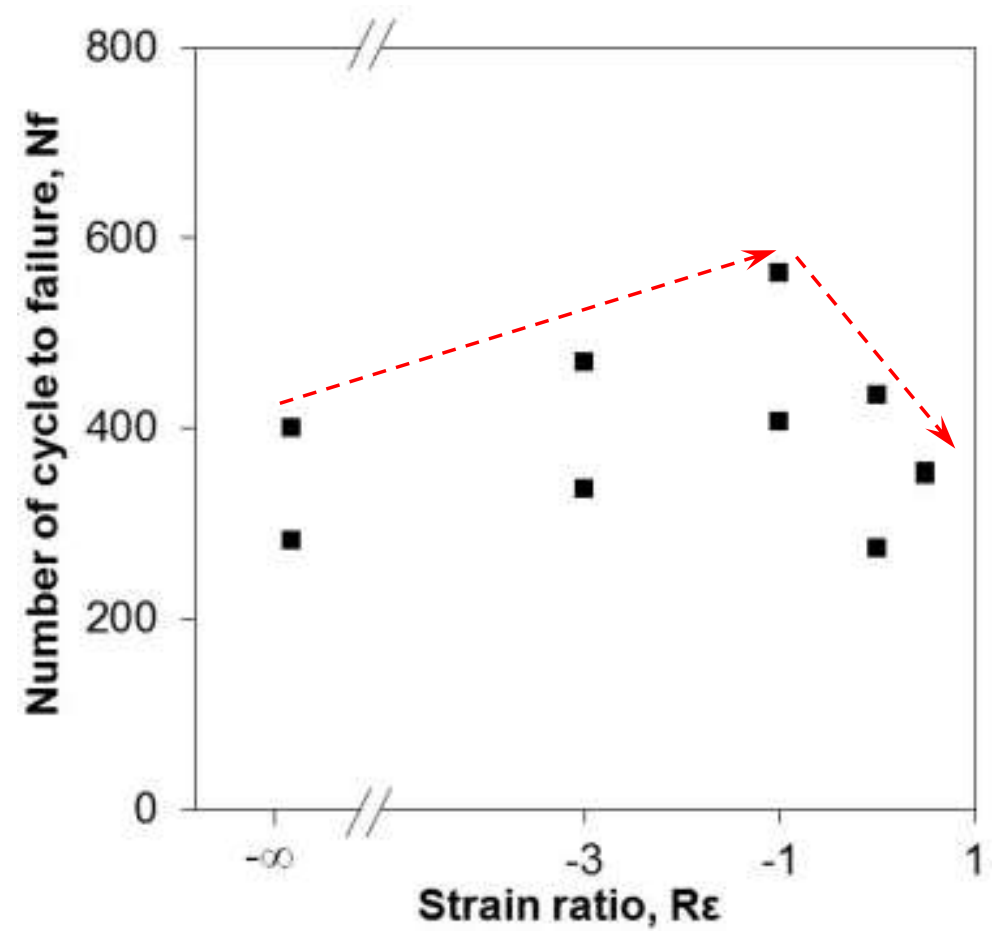

Fig. 6.3 Number of cycles to failure vs. strain ratio for the rolled ZEK100 alloy tested at a total strain amplitude of $0.8 \%$ and a strain rate of $1 \times 10^{-2} \mathrm{~s}^{-1}$. 


\subsection{Fractography}

Fig.6.4 shows an overall view of the fracture surfaces of the specimens fatigued at $R_{\varepsilon}=0.5$ (Fig.6.4(a)) and $R_{\varepsilon}=-\infty$ (Fig.6.4(b)). Fatigue crack propagation area was greater for lower strain ratio (i.e., $R_{\varepsilon}=-\infty$ ). This can be attributed to the lowest maximum tensile stress and the smallest mean stress at $R_{\varepsilon}=-\infty$. This can also be related to the stress amplitude at $R_{\varepsilon}=-\infty$ which was the lowest among all the tests, delaying the onset of rapid propagation where the maximum stress intensity reached the fracture toughness of the alloy.
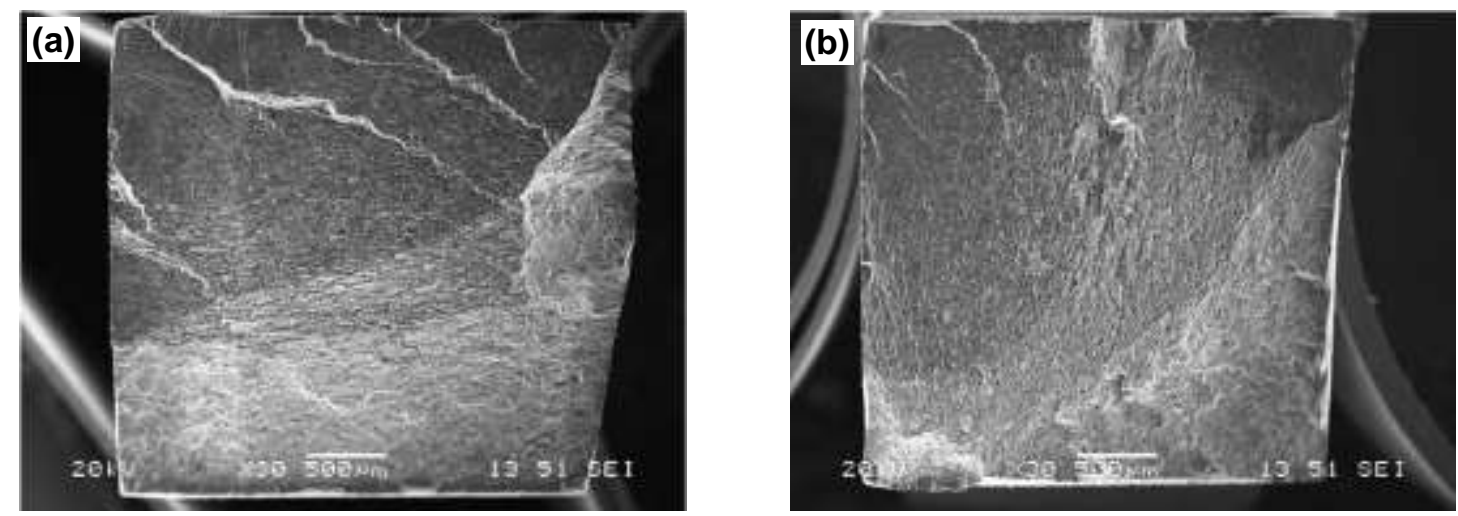

Fig. 6.4 Typical SEM images of the overall fracture surfaces of the specimens fatigued at total strain amplitude of $0.8 \%$ and at a strain ratio of (a) 0.5 and (b) $-\infty$.

\subsection{Quantitative analysis of the anelastic behavior}

As previously stated, ZEK100 exhibited some degree of asymmetry in the stress-strain hysteresis loops in tension and compression (Fig.6.1), which was related to the occurrence of twinningdetwinning process and the related non-linear elastic (or pseudo-elastic or anelastic) behavior [124,125], where an eccentricity parameter $e$ used to quantify hysteresis loop asymmetry was 
defined as a horizontal "gap" in terms of strain amount by which the intersection of a line connecting the upper and lower peaks of the loop (i.e., points $A$ and $B$, respectively, as shown in Fig.6.5) with the strain axis, is shifted from the coordinate origin. It is noted that the eccentricity could also be quantified vertically; however, the horizontal information is used to avoid redundancy.

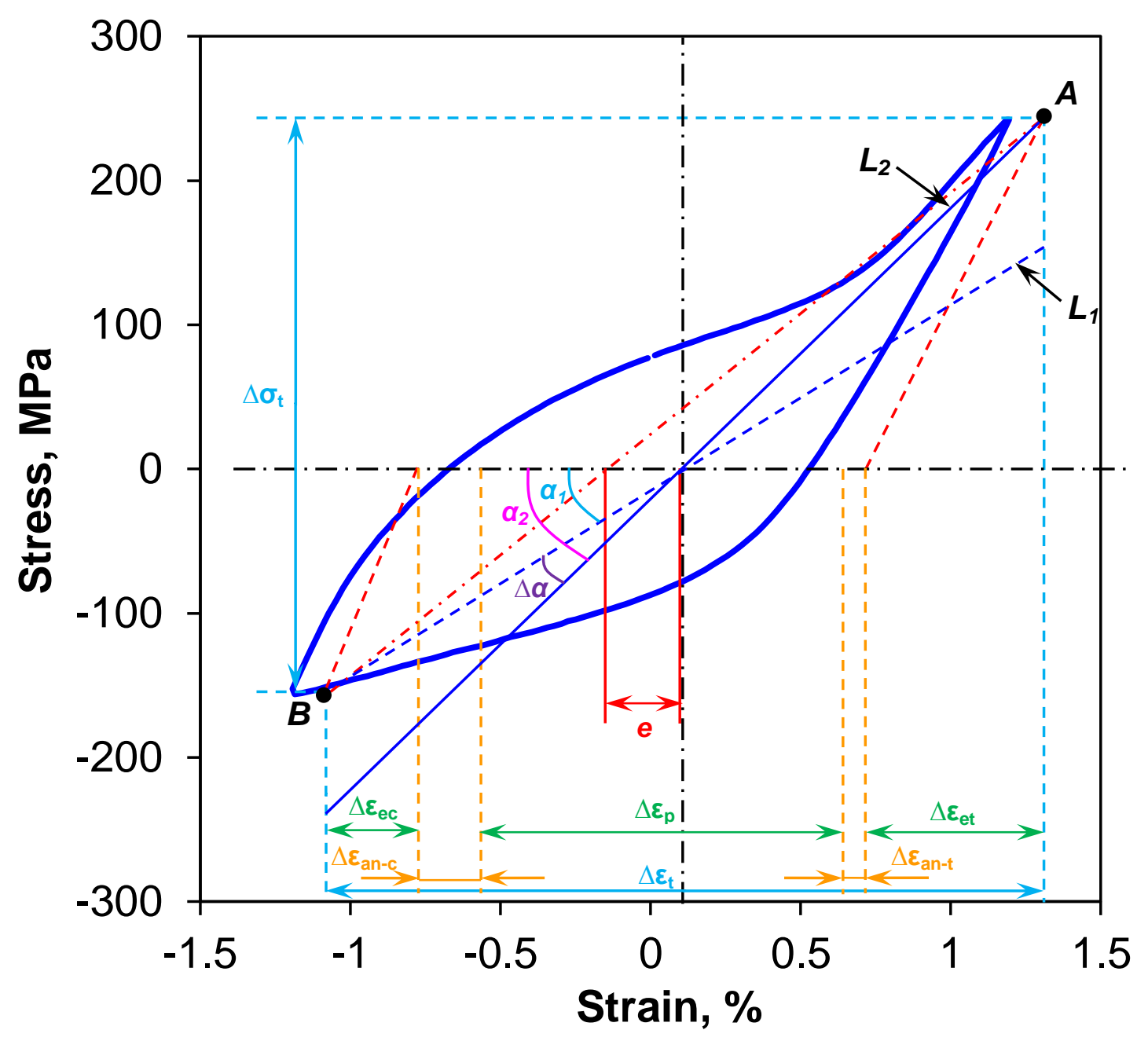

Fig. 6.5 Schematic illustration showing the parameters defined to characterize the anelastic behavior in tension and compression by means of a stress-strain hysteresis loop. 
Two more parameters illustrated in Fig.6.5 are the angle deviation $\Delta \alpha$ and a relative slope change $d$ which are defined as [124],

$$
\begin{gathered}
\Delta \alpha=\alpha_{2}-\alpha_{1}, \\
d=\left|\frac{S_{2}-S_{1}}{S_{1}}\right| \times 100,
\end{gathered}
$$

where $\alpha_{1}$ is the angle between $L_{1}$ and the horizontal axis, $\alpha_{2}$ is the angle between $L_{2}$ and the horizontal axis, $S_{1}$ and $S_{2}$ are the slopes of lines $L_{1}$ and $L_{2}$, respectively (Fig.6.5).

\subsubsection{Effect of strain ratio on the anelastic behavior}

Fig.6.6(a) shows the change of the eccentricity $e$ along the strain axis for the 2 nd and mid-life cycles, respectively. It is seen that the eccentricity decreased with increasing strain ratio, indicating more symmetric hysteresis loops. It is clear that a zero eccentricity value corresponds to a symmetric hysteresis loop, e.g., in the fcc metals [129]. Fig.6.6(b) shows the relative slope

change $d$ for the $2^{\text {nd }}$ cycle and mid-life cycle, respectively. Similarly, the mid-life cycle tended to be slightly flatter, and the increase in the strain ratio led to a decrease in $d$, indicating a better hysteresis symmetry characteristic. Fig.6.5(c) corroborates the above definitions since similar trends were observed for the angle deviation $\Delta \alpha$. Results in Fig.6.6 are directly related to the mechanisms of the material response to cyclic deformation since they express the evolution of the anelastic behavior and the degree of asymmetry of the hysteresis loops. In fact, during fatigue tests at various strain ratios, the twin density varies as twinning and detwinning interchange by alternating activities. In Fig.6.6 it was observed that with decreasing strain ratio, all the asymmetry quantifying parameters increased, confirming the presence of more asymmetric loops. In terms of the response of the alloy, this could be attributed to the high compressive loading 
along the RD and the favorable condition for extension twinning, which would grow and shrink with the increase and decrease of the applied loads leading to an accumulation of residual twins to be discussed in next section.
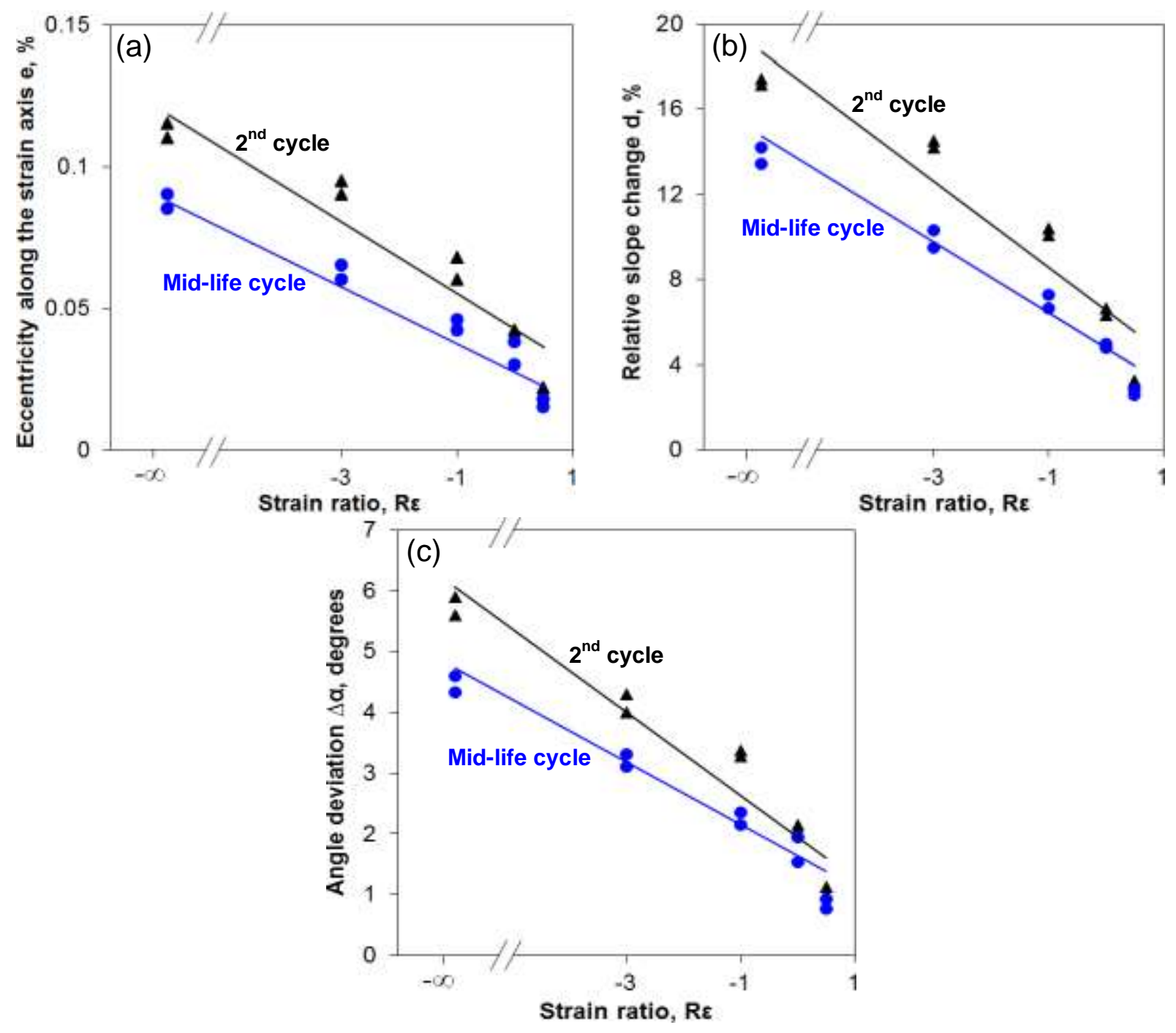

Fig. 6.6 (a) Eccentricity along the strain axis $e$, (b) relative slope change $d$, and (c) angle deviation $\Delta \alpha$ as a function of strain ratio for ZEK100 tested at a strain rate of $1 \times 10^{-2} \mathrm{~s}^{-1}$ at a total strain amplitudes of $0.8 \%$ for the second and mid-life cycles, respectively. 


\subsection{Distribution of residual twins}

Fig.6.7(a) and (b) show optical micrographs in the areas near fracture where the distribution of residual twins is presented for $R_{\varepsilon}=0.5$ and $R_{\varepsilon}=-\infty$ respectively. A large number of residual twins was observed at both strain ratios, which can be related to the insufficiency of slip systems in $\mathrm{Mg}$ at RT. With decreasing strain ratio, twin formation increased due to intense compressive loading along RD. Twinning is therefore predominant through cyclic deformation, especially at lower strain ratios. A lesser extent of twinning was observed in [75] for the GW103K Mg alloy (10 wt.\% Gd and 3 wt.\% Y), which was a richer RE-containing alloy compared with the current ZEK100 $(0.2 \mathrm{wt} . \% \mathrm{Nd})$. The lack of major twin deformation was linked to the fine grain size and REcontaining precipitates [75]. Gharghouri et al. [50] stated that the anelastic effect in $\mathrm{Mg}$ alloys is a consequence of the growth and shrinkage of the $\{10 \overline{1} 2\}\langle 10 \overline{1} 1\rangle$ extension twins with the increase and decrease of the applied load.
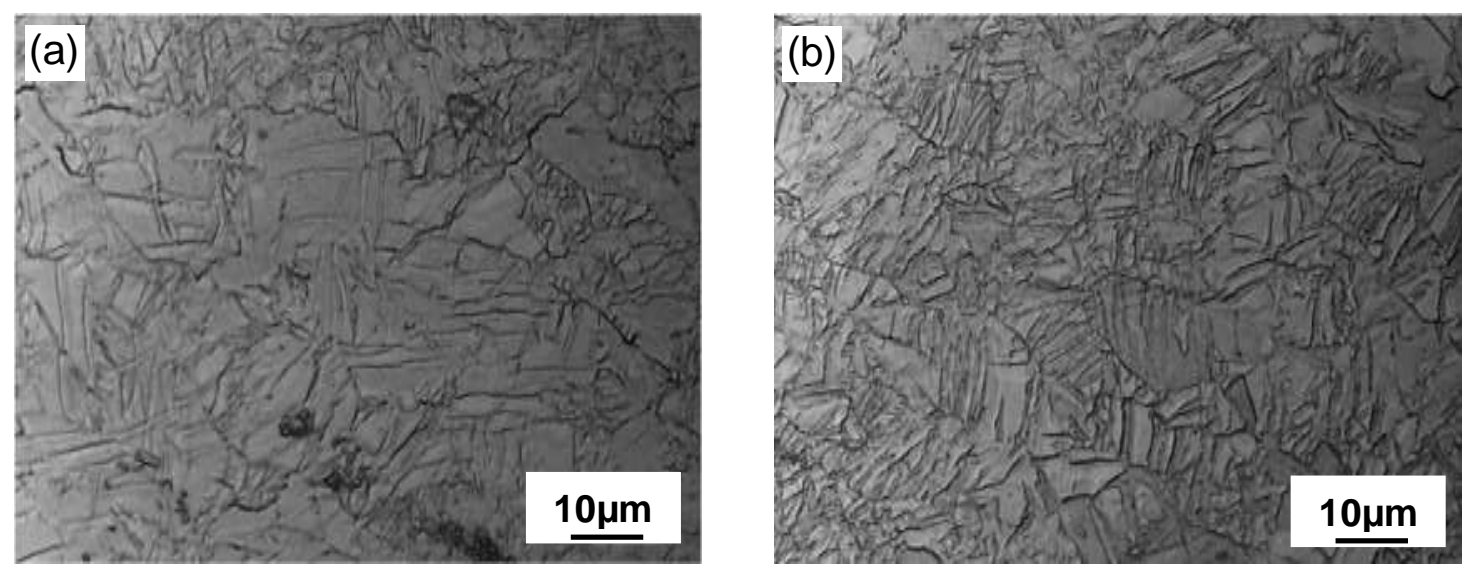

Fig.6.7 Micrographs of the areas near the fracture surface fatigued at (a) $R_{\varepsilon}=0.5$, and (b) $R_{\varepsilon}=-\infty$. 


\subsection{Sensitivity analysis of the anelastic behavior}

\subsubsection{Theoretical foundation}

For the best case scenario of ideal and symmetrical hysteresis loops (e.g., for most fcc alloys), the total strain range $\left(\Delta \varepsilon_{t}\right)$ would basically be [130],

$$
\Delta \varepsilon_{t}=\Delta \varepsilon_{e}+\Delta \varepsilon_{p}
$$

where $\Delta \varepsilon_{e}$ is the total elastic strain range and $\Delta \varepsilon_{p}$ is the total plastic strain range. Meanwhile, the total elastic strain range could be defined as a combination of,

$$
\Delta \varepsilon_{e}=\Delta \varepsilon_{e-t}+\Delta \varepsilon_{e-c}
$$

where $\Delta \varepsilon_{e-t}$ is the tensile elastic strain range and $\Delta \varepsilon_{e-c}$ is the compressive elastic strain range. In the event of tension-compression asymmetry, e.g., in wrought $\mathrm{Mg}$ alloys, Equ.(6.3) needs to be modified to account for the anelastic behavior,

$$
\Delta \varepsilon_{t}=\Delta \varepsilon_{e}+\Delta \varepsilon_{p}+\Delta \varepsilon_{a n}
$$

where $\Delta \varepsilon_{a n}$ is the total anelastic strain range, which can be further expressed as,

$$
\Delta \varepsilon_{a n}=\Delta \varepsilon_{a n-t}+\Delta \varepsilon_{a n-c}
$$

where $\Delta \varepsilon_{a n-t}$ is the tensile anelastic strain range and $\Delta \varepsilon_{a n-c}$ is the compressive anelastic strain range.

Throughout the present investigation, strain ratio has been observed to influence considerably the anelastic behavior. However its impact within a set of influencing parameters is still unclear. Sensitivity analysis would alloy to see whether it persists as the most influencing parameter or fades in comparison with other input factors. Fig.6.8 represents a simplified algorithm of the global sensitivity analysis performed in order to narrow down the most influencing factors on the 
anelastic behavior. Two different input categories were differentiated. The controllable input (i.e., $R_{\varepsilon}$ ) can be defined prior to testing and can be varied by the user as desired. The uncontrollable inputs that actually result from the material behavior and microalloying, and are directly linked to fatigue life, strength and ductility related parameters $\left(N_{f}, \sigma_{f}^{\prime}, b, \varepsilon_{f}^{\prime}\right.$ and $\left.c\right)$.

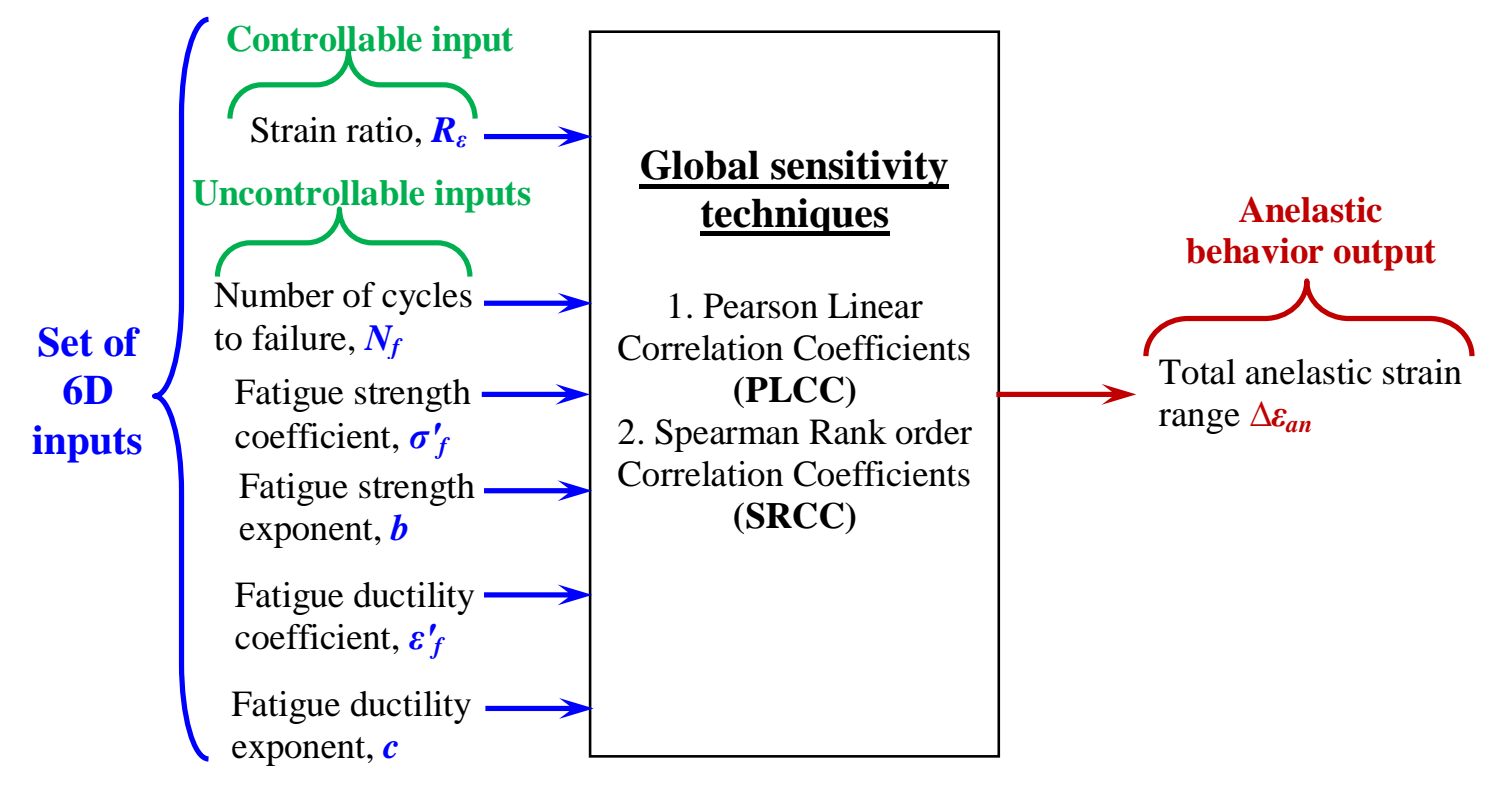

Fig.6.8 Representation of the global sensitivity analysis performed to identify the most influencing factors on the anelastic behavior.

\subsubsection{Sensitivity model}

A 6D set of input factors was listed in Table 6.1, where the range of variation for each input was based on the literature $[75,120,124,130] . R_{\varepsilon}$ actually varied from $-\infty$ to +0.5 , however quantifiable were chosen the sensitivity analysis (a lower limit of -10).

Table 6.1 Six-dimensional input factor set for sensitivity analysis of the anelastic behavior. 


\begin{tabular}{cc}
\hline Input & Range of variation \\
\hline Strain ratio, $R_{\varepsilon}$ & {$[-10-0.5]$} \\
Number of cycles to failure, $N_{f}$ & {$[200-600]$} \\
Fatigue strength coefficient, $\sigma_{f}^{\prime}, \mathrm{MPa}$ & {$[500-700]$} \\
Fatigue strength exponent, $b$ & {$[-0.16--0.05]$} \\
Fatigue ductility coefficient, $\varepsilon_{f}^{\prime}$ & {$[0.01-0.2]$} \\
Fatigue ductility exponent, $c$ & {$[-0.7--0.4]$} \\
\hline
\end{tabular}

Input factors in Table 6.1 have a wide variety of ranges. Hence, the variability of any input factor $X$ was scaled into $\tilde{X}$, which varies within a range in-between 0 to 1 . Thus, knowing the actual physical range of each input factor $\left[X_{\min }-X_{\max }\right]$, the following normalization was defined,

$$
\tilde{X}=\frac{X-X_{\min }}{X_{\max }-X_{\min }}
$$

Monte Carlo sampling was performed with $10^{6}$ iterations to ensure that convergence was reached when constructing the input data set. Based on Equ.(6.5), the anelastic strain amplitude was as,

$$
\frac{\Delta \varepsilon_{a n}}{2}=\frac{\Delta \varepsilon_{t}}{2}-\frac{\Delta \varepsilon_{e}}{2}-\frac{\Delta \varepsilon_{p}}{2}
$$

As previously see in Equ.(5.1), elastic strain amplitude is expressed using the Basquin's equation, and plastic strain amplitude using the Coffin-Manson relation,

$$
\begin{aligned}
& \frac{\Delta \varepsilon_{e}}{2}=\frac{\sigma_{f}^{\prime}\left(2 N_{f}\right)^{b}}{E} \\
& \frac{\Delta \varepsilon_{p}}{2}=\varepsilon_{f}^{\prime}\left(2 N_{f}\right)^{c}
\end{aligned}
$$


Substituting Equ.(6.9) and (6.10) into Equ.(6.8), only the total strain amplitude $\frac{\Delta \varepsilon_{t}}{2}$ is left to be expressed as a function of one of the input variables presented in Fig.6.8 and Table 6.1. This quantity was meant to be constant at $0.8 \%$ in the present tests; however it was affected by an experimental uncertainty, which is common in mechanical testing when introducing controllable quantities $[131,132]$. The uncertainty on the total strain amplitude was monitored and tended to be a function of strain ratio, i.e., $\frac{\Delta \varepsilon_{t}}{2}=f\left(R_{\varepsilon}\right)$. Fig.6.9 shows a slight variation of total strain amplitude for mid-life cycles, corresponding to a relative error below the specified $2.5 \%$ even down to $-\infty$ (Fig.6.9(b)), based on the Instron 2620 series of dynamic strain gauge extensometer designed to meet the requirements of ASTM E83.
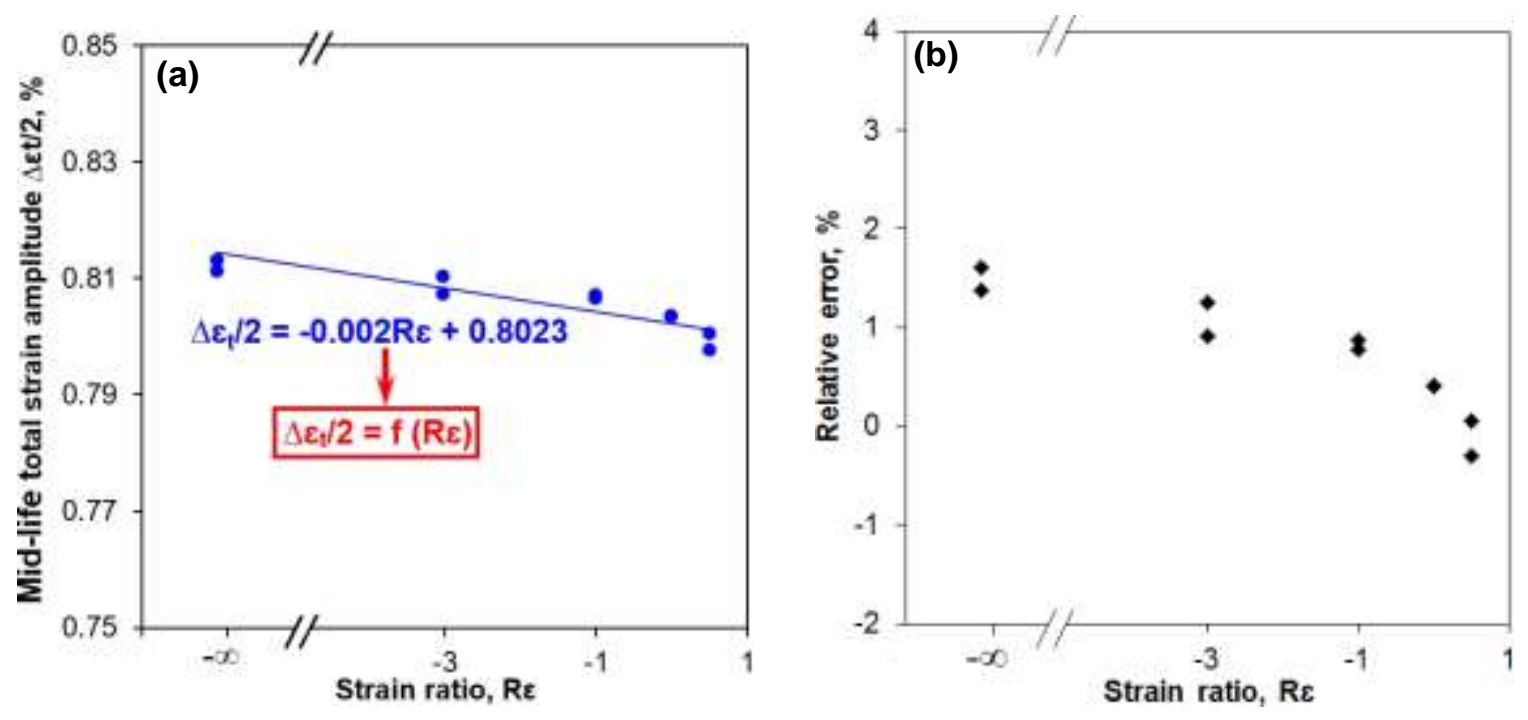

Fig.6.9 (a) Mid-life total strain amplitude, and (b) relative error as a function of strain ratio in the fatigue tests of ZEK100 alloy at a strain rate of $1 \times 10^{-2} \mathrm{~s}^{-1}$.

Using all above information, the anelastic strain amplitude $\Delta \varepsilon_{a n} / 2$ was defined as, 


$$
\frac{\Delta \varepsilon_{a n}}{2}=\frac{\Delta \varepsilon_{t}}{2}-\frac{\Delta \varepsilon_{e}}{2}-\frac{\Delta \varepsilon_{p}}{2}=f\left(R_{\varepsilon}\right)-\frac{\sigma_{f}^{\prime}\left(2 N_{f}\right)^{b}}{E}-\varepsilon_{f}^{\prime}\left(2 N_{f}\right)^{c},
$$

which groups the six controllable and uncontrollable input factors $R_{\varepsilon}, N_{f}, \sigma_{f}^{\prime}, b, \varepsilon_{f}^{\prime}$ and $c$ (with a constant Young's modulus of $E=44.9 \mathrm{GPa}$ ). Two global sensitivity techniques were used in our analysis, beginning with the Pearson linear correlation coefficient (PLCC), which provides a measure of the linear relationship between two sets of variables. For a response $Y$ and a set of inputs $X$, the PLCC can be written as [133],

$$
\rho_{X Y}=\frac{\sum_{i=1}^{N}\left(y_{i}-\bar{y}\right)\left(x_{i}-\bar{x}\right)}{\sqrt{\sum_{i=1}^{N}\left(y_{i}-\bar{y}\right)^{2} \sum_{i=1}^{N}\left(x_{i}-\bar{x}\right)^{2}}},
$$

where $\bar{x}$ and $\bar{y}$ are the means of $X$ and $Y$, respectively.

The PLCC was evaluated between the 6D input factors and the anelastic strain amplitude, as summarized in Fig.6.10(a). It is seen that the most influencing parameter was $R_{\varepsilon}$ (PLCC=0.6005). The second strong was the fatigue ductility exponent $c$ (PLCC $=-0.4277)$, followed by the fatigue ductility coefficient $\varepsilon_{f}^{\prime}(\mathrm{PLCC}=-0.3395)$. It was also observed that the two inputs related to the strength of the material (fatigue strength coefficient $\sigma_{f}^{\prime}$ and the fatigue strength exponent $b$ ) came next, with a lesser degree of negative correlation (PLCC $=-0.3289$ and -0.2812 , respectively). This reflects that both strength $\left(\sigma_{f}^{\prime}\right.$ and $b$ ) and ductility ( $\varepsilon_{f}^{\prime}$ and $c$ ) parameters had a comparatively similar impact on the anelastic behavior of the material (i.e., negative correlations in the range 0.25-0.45). The previous suggests the effectiveness of $\mathrm{Nd}$ addition in ZEK100 along with the proper processing and annealing, giving rise to a fairly good combination of strength and ductility. The least influencing parameter was the number of cycles to failure $N_{f}$ since it was mainly absorbed by the exponents $b$ and as seen in Equ.6.11. 
To verify the previous and remedy the potential disadvantage of PLCC in the event of non-linear relationships, providing the monotonicity condition, the Spearman rank order correlation coefficient (SRCC) were also evaluated [134]. Data were replaced by their corresponding rank, which involves first putting the values in a numerical order and then assigning them new indices to identify where they fall in the ordered set. Let $r$ be the ordered matrix of the 6 inputs and $s$ be the ordered vector of the output, the SRCC can be expressed as follows [134],

$$
\rho_{s r}=\frac{\sum_{i=1}^{N}\left(r_{i}-\bar{r}\right)\left(s_{i}-\bar{s}\right)}{\sqrt{\sum_{i=1}^{N}\left(r_{i}-\bar{r}\right)^{2} \sum_{i=1}^{N}\left(s_{i}-\bar{s}\right)^{2}}},
$$

where $\bar{r}$ and $\bar{s}$ are the means of $s$ and $r$, respectively.
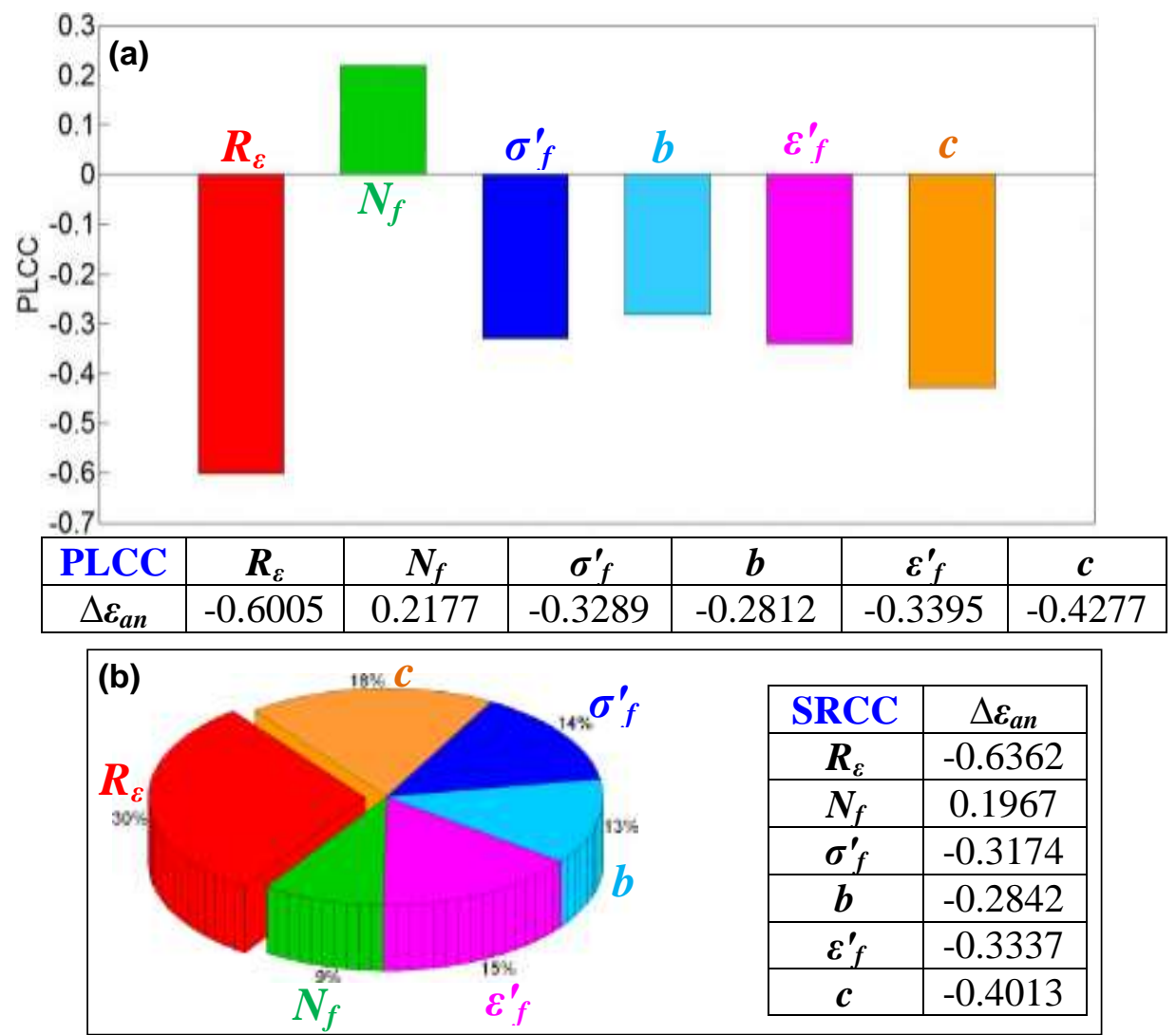

Fig.6.10 Influencing inputs on the anelastic behavior by means of (a) Pearson Linear Correlation Coefficients (PLCC), and (b) Spearman Rank order Correlation Coefficients (SRCC). 
A pie chart was generated in Fig.6.10(b) for the SRCC, and contribution of each input to the anelastic behavior was presented in terms of percentile proportions, corroborating well the findings of the above PLCC. $R_{\varepsilon}$ with a $30 \%$ contribution in a negative correlation persisted as the most influencing.

\subsection{Summary}

1. During cyclic deformation, significant plastic deformation occurred in the tensile phase of the first cycle at $R_{\varepsilon}=0$ and 0.5 due to the stipulated maximum strain. Asymmetry of hysteresis loops initially present tended to diminish towards mid-life cycles at different strain ratios.

2. With increasing strain ratio, fatigue life was observed to first increase, reach its maximum at a strain ratio of $R_{\varepsilon}=-1$, and then decrease. This was a result of the coupled role of cyclic stress amplitude and plastic strain amplitude which were inversely affected by the strain ratio.

3. The mean stress at $R_{\varepsilon}=-1$ remained nearly zero and invariant. The closer the strain ratio to $R_{\varepsilon}=-1$ was, the lower the absolute value of mean stress was, which would also lead to a higher fatigue life. The mean stress relaxation occurred more significantly in the initial stage of cyclic deformation especially for the strain ratio more remotely from $R_{\varepsilon}=-1$.

4. Despite the decrease of the anelastic behavior in this alloy, it still largely remained due to the occurrence of twinning and detwinning. With decreasing strain ratio, the extent of twin formation increased. Global sensitivity analysis indicated that strain ratio was an influential parameter for the anelastic behavior. The anelastic strain amplitude, along with the three newly defined parameters (eccentricity, angle deviation, and relative slope change) all increased with decreasing strain ratio, reflecting more asymmetric and skewed loops. 


\section{CHAPTER 7}

\section{DEFORMATION AND STRENGTHENING MECHANISMS OF 2024AI ALLOY AND 2.0 WT.\% CNT REINFORCED 2024AI COMPOSITE}

\subsection{Introduction}

The main objectives of this study were to examine the microstructural features coming from the CNT addition, evaluate the compressive deformation behavior of the 2.0 wt.\% CNT/2024Al composite at varying strain rates and temperatures, and identify the salient strengthening mechanisms.

\subsection{Microstructure and EBSD analysis}

Fig.7.1 shows the SEM micrographs for the 2024Al (Fig.7.1(a) and (b)) and the 2.0 wt.\% CNT/2024Al (Fig.7.1(c) and (d)). Both base alloy and composite displayed some coarser particles indicated by the thicker yellow arrows on Fig.7.1(b) and (d). However, a close examination of Fig.7.1(c) and (d) revealed two types of particles which could be distinguished by their distinct size and shape. Numerous uniformly distributed nanoparticles in the $\mathrm{Al}$ matrix were seen in the composite (as pointed out by the thinner red arrows in Fig.7.1(d)), which will be further examined via TEM analysis. To understand the nature of coarser particles in the composite, EDS line scan was performed and shown in Fig.7.2. During the EDS analysis all the main alloying elements (i.e., $\mathrm{Al}, \mathrm{Cu}, \mathrm{Mg}$, and $\mathrm{Mn}$ ) were considered, however only $\mathrm{Cu}$-containing particles were present (Fig.7.2(b)). 

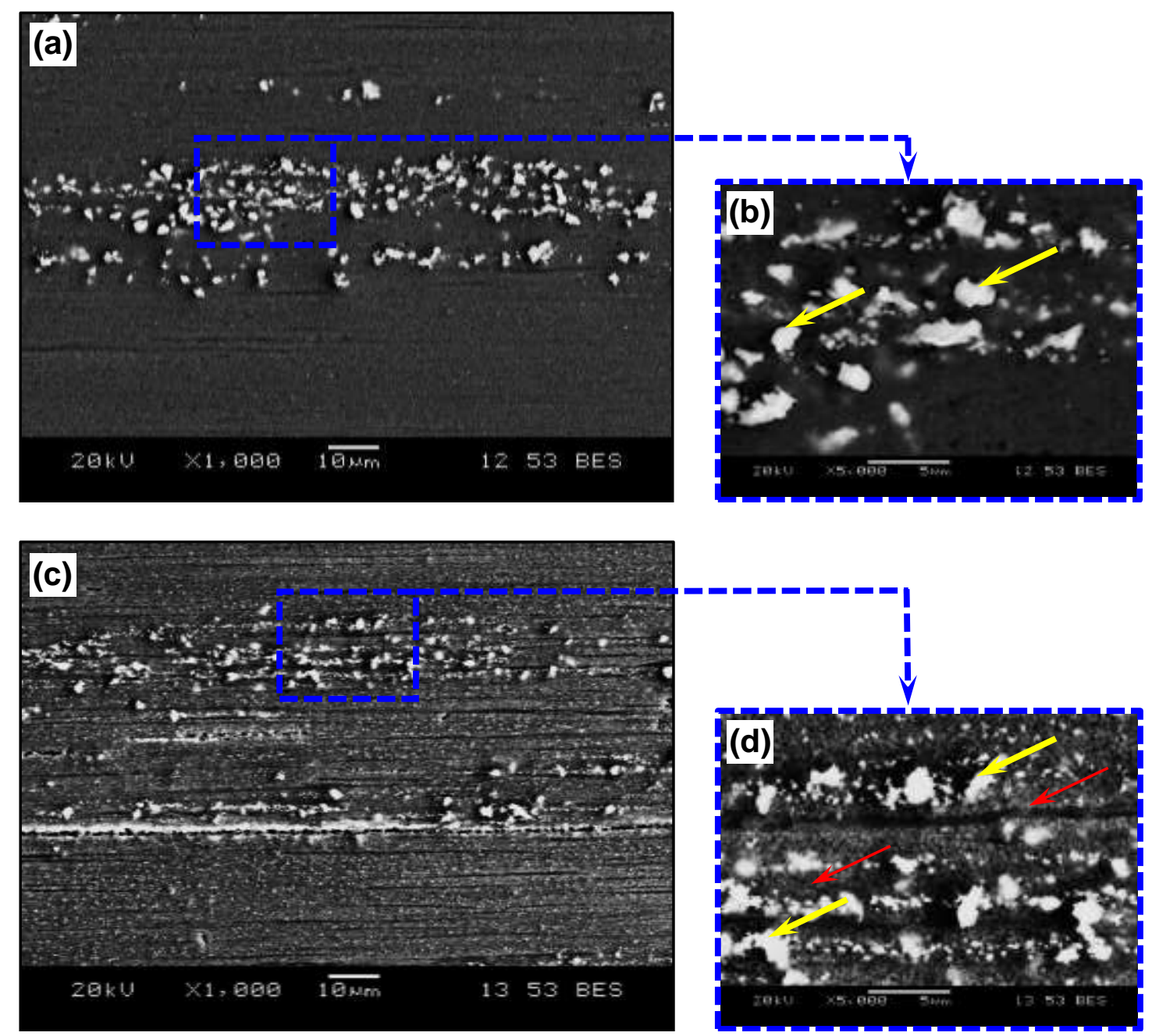

Fig.7.1 Typical SEM micrographs showing the details of microstructural features of the 2024Al alloy and 2.0 wt.\% CNT/2024Al composite at (a, c) lower and (b, d) higher magnifications, respectively. Yellow coarse arrows indicate $\mathrm{Cu}$-containing particles, and red thin arrows designate nanoparticles.

Similar results were reported by Bustamante et al. [27] who studied the microstructure of a 5.0 wt.\% CNT/2024Al composite after sintering, and observed a good dispersion of $\mathrm{Al}_{2} \mathrm{Cu}$ coarse particles due to the low cooling rate in the sintering process. The presence of this phase will be further confirmed via XRD phase analysis. The eventual presence of $\mathrm{Al}_{2} \mathrm{O}_{3}$ oxides due to the spontaneous reaction between $\mathrm{Al}$ and $\mathrm{O}_{2}$, when exposed to air during milling was also acknowledged and will be further discussed in the coming sections. 


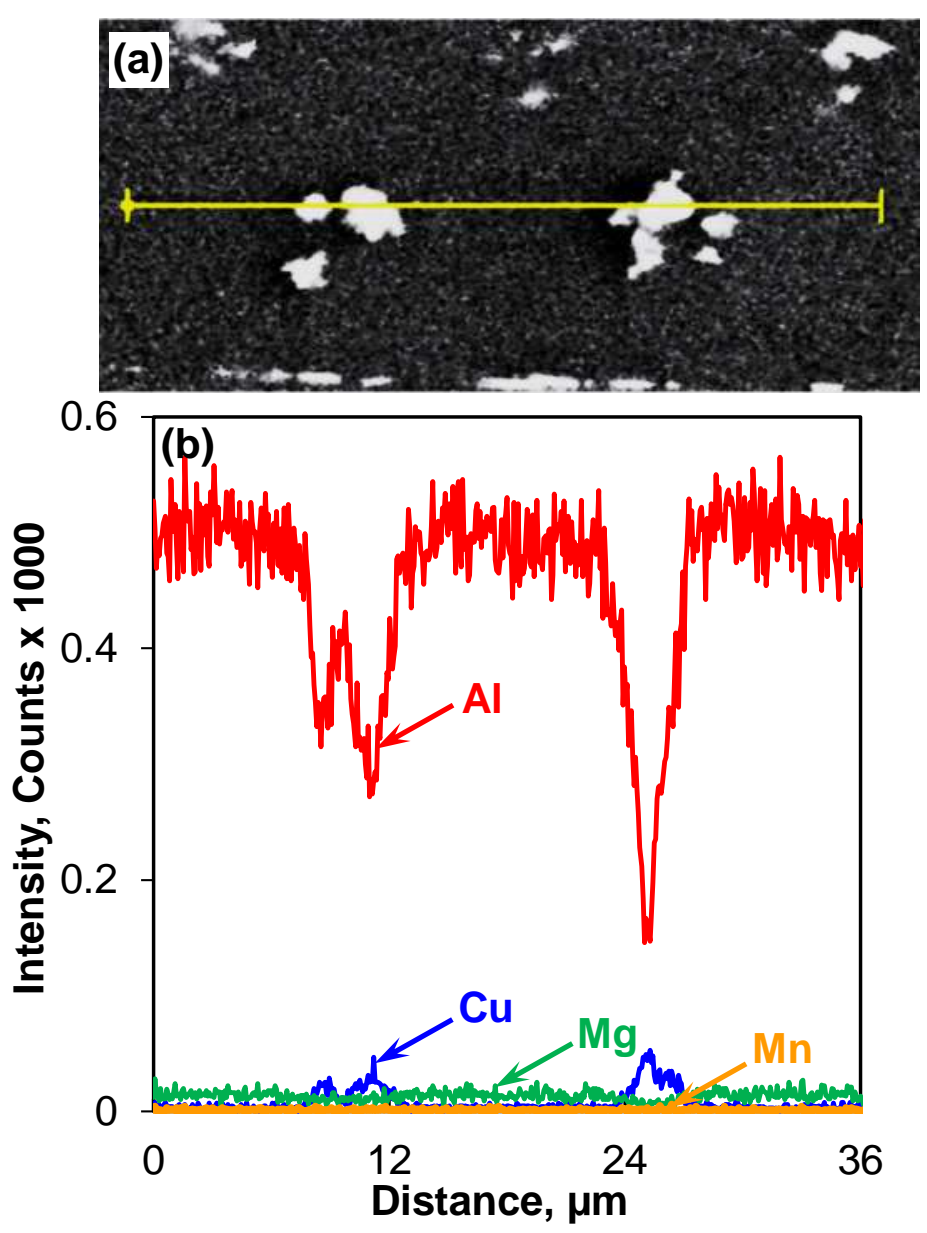

Fig.7.2 (a) A typical SEM back-scattered electron image, and (b) EDS line scan results across a position indicated by the line in (a) for the $2.0 \mathrm{wt} . \% \mathrm{CNT} / 2024 \mathrm{Al}$ composite.

TEM examinations on the $2.0 \mathrm{wt} . \% \mathrm{CNT} / 2024 \mathrm{Al}$ composite revealed singly dispersed CNTs in the $\mathrm{Al}$ matrix, as indicated by the red thin arrows in Fig.7.3(a). The original size of the CNTs was $>5 \mu \mathrm{m}$ in length and 10 20 $\mathrm{nm}$ in diameter, hence, CNTs in Fig.7.3(a) were observed to be shorter than the as-received ones. This could be attributed to the significant breakup in the fabrication of the composite where CNTs were shortened during ball milling due to shear effect. CNTs in Fig.7.3(a) were also seen to be homogeneously dispersed where no cluster or localized agglomerations were detected. The presence of $\mathrm{Al}_{4} \mathrm{C}_{3}$ phase was also perceived, as indicated by the blue thick arrows in Fig.7.3(a), being either directly attached to CNTs or in the matrix in the 
vicinity of CNTs. The existence of this phase will be further verified via XRD analysis. Fig.7.3(b) presents a HRTEM image showing the interface between the Al matrix and CNTs, where different features (i.e., $\mathrm{Al}, \mathrm{CNT}$, and interface) were clearly distinguished. The CNT-Al interface was densely bonded, and no void or defects were observed. The layered carbon structure within the CNTs and the interlayer distance between the CNT walls were visible. The presence of such a coherent interface indicates the direct bond between the matrix and the reinforcements which could be linked to the load transfer mechanism, where CNTs would carry a portion of the load between the matrix and the reinforcements [135]. This would influence the strengthening mechanisms of the composite where interfacial shear stresses originating from the matrix could be transferred to the CNTs.
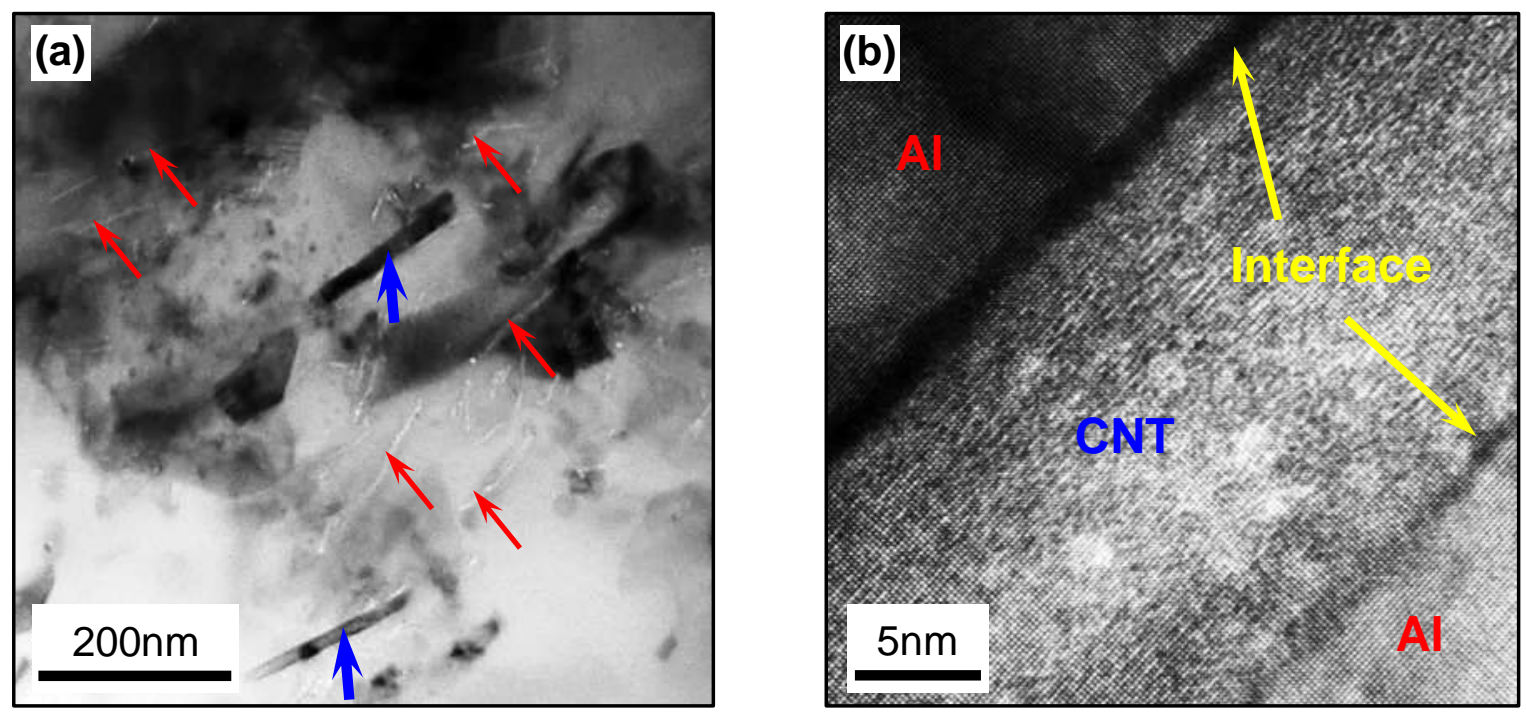

Fig.7.3 (a) A typical TEM image of the $2.0 \mathrm{wt} . \% \mathrm{CNT} / 2024 \mathrm{Al}$ composite. Red thin arrows indicate singly dispersed CNTs, and blue thick arrows designate the $\mathrm{Al}_{4} \mathrm{C}_{3}$ phase, (b) HRTEM image showing the interface structure between the $\mathrm{Al}$ matrix and CNTs. 
Fig.7.4(a) and (b) display the EBSD orientation maps for the 2024Al alloy and the 2.0wt.\% CNT/2024Al composite, where the map color legend was projected towards the ED. Larger elongated grains in both alloy and composite mainly exhibited a $<111>$ texture (blue color), with a few elongated grains orientated in the $<001>$ direction (red color). Equiaxed grains present in Fig.7.4(a) and (b) revealed more randomized orientations or assorted colors. Likewise, EBSD maps of laminated CNT/7055Al composite were studied in [136] and the presence of a strong $<111>$ fiber texture was reported. In fact, this orientation was acknowledged as a hard orientation in the fcc structures, in direct relation to the normal $\{111\}<110>$ slip systems [5]. Elongated grains present in Fig.7.4(a) and (b), along the ED were credited to the severe plastic deformation during extrusion.
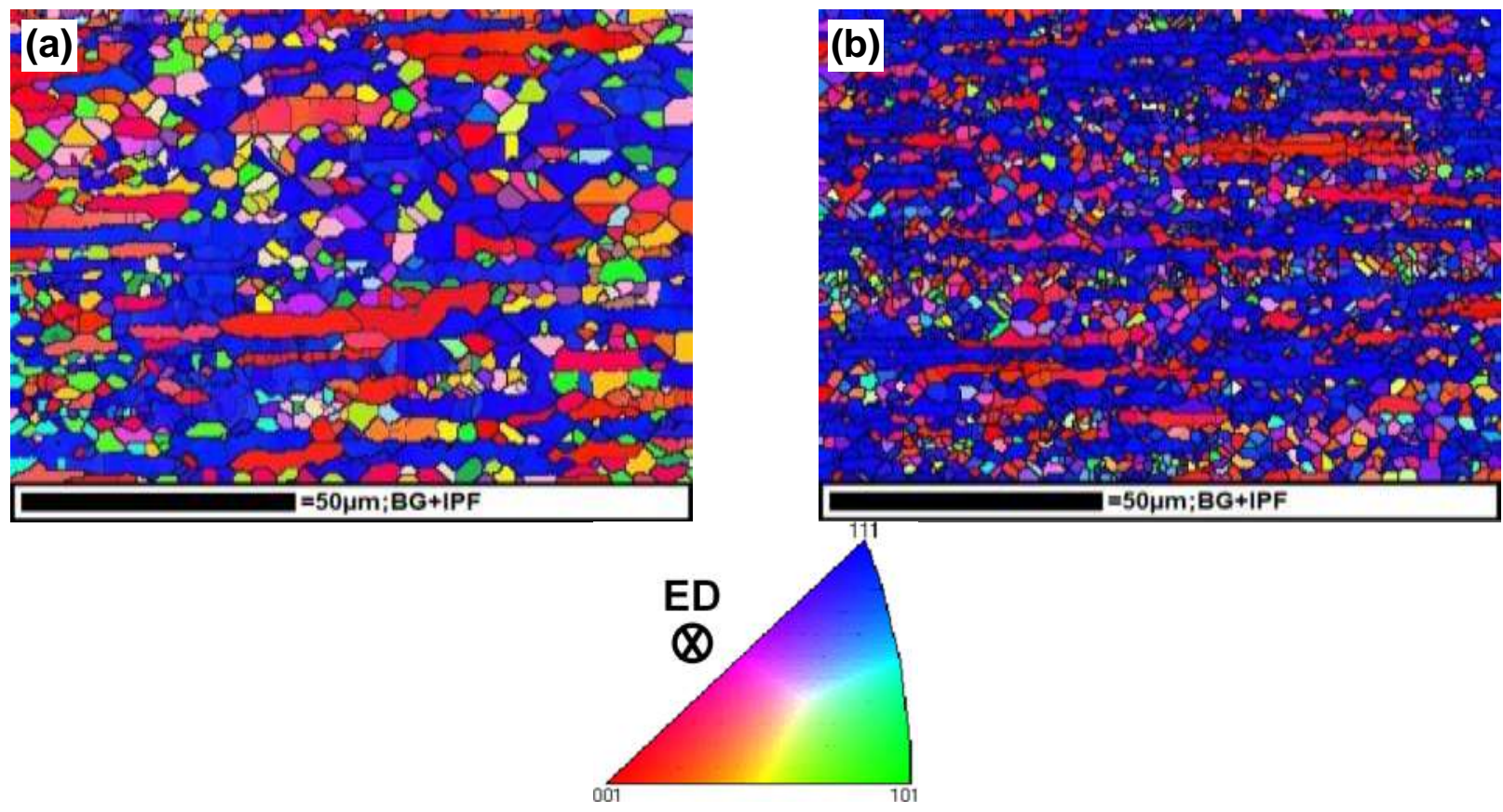

Fig.7.4 EBSD orientation maps of (a) the 2024Al alloy, and (b) the $2.0 \mathrm{wt} \% \mathrm{CNT} / 2024 \mathrm{Al}$ composite. 
Since Fig.7.4(a) and (b) were provided under the same magnification, direct conclusions could be drawn about the major grain refinement achieved in the composite. The grain refinement due to CNT addition was recognized in literature for MMCs $[95,137,138]$. Second-phase particles previously seen observed in Fig.7.1(a) and (b) (nano-sized CNTs and Cu-containing particles) played a major role in controlling grain size, stimulating the nucleation of recrystallization and in pinning boundaries. CNTs in the composite caused a motion restriction of GBs by acting as pinning forces prohibiting the grain growth and leading to the grain refinement observed.

\subsection{X-ray diffraction analysis}

XRD patterns acquired from the alloy and composite are presented in Fig.7.5. The presence of Al and $\mathrm{A} 12 \mathrm{Cu}$ peaks could be clearly observed in both materials.

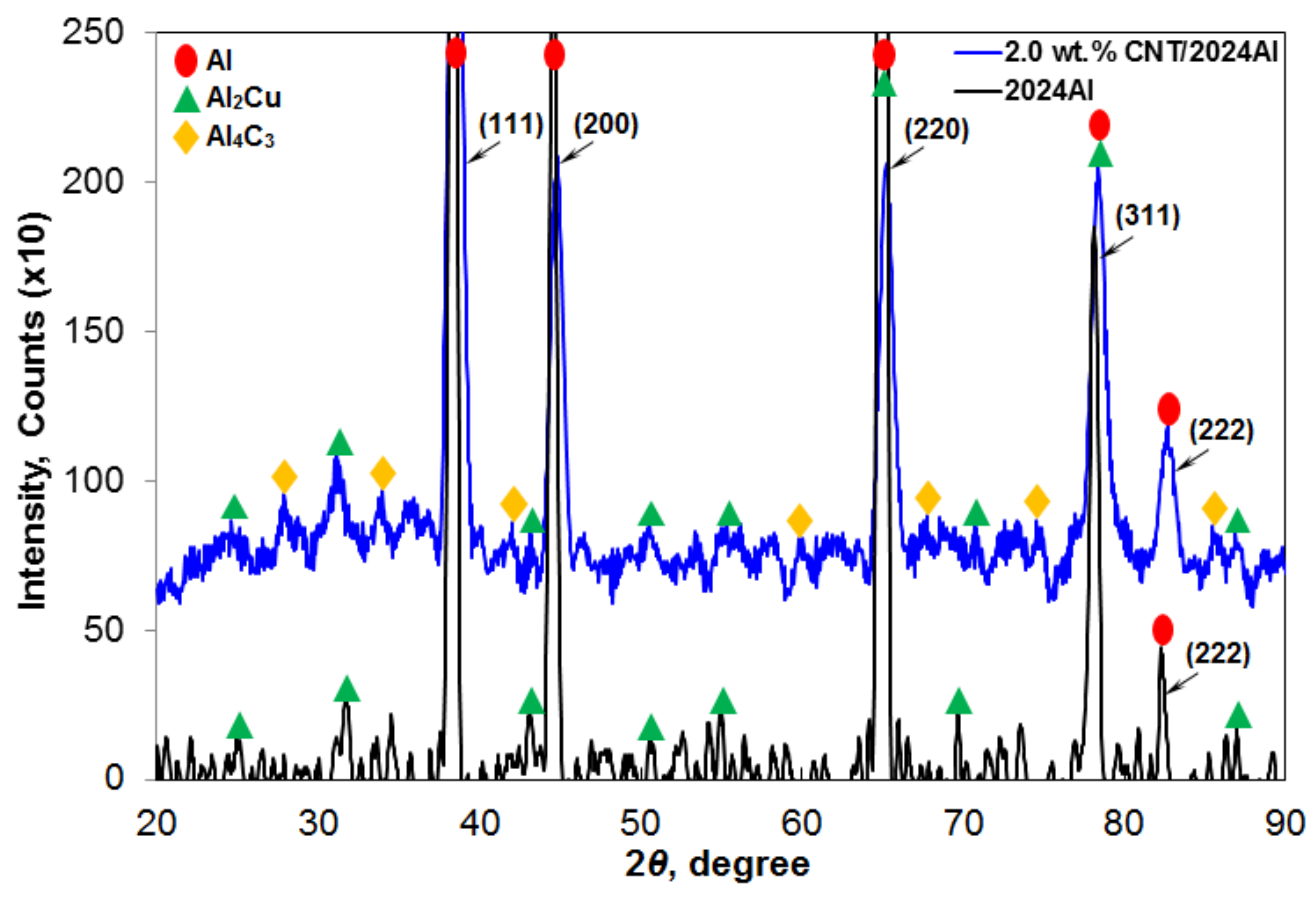

Fig.7.5 X-ray diffraction patterns of the 2024Al alloy and 2.0 wt.\% CNT/2024Al composite. 
Some weak $\mathrm{Al}_{4} \mathrm{C}_{3}$ peaks could also be identified from the XRD pattern of the composite, being in agreement with the TEM observations shown in Fig.7.3(a). No carbon peaks were detected in the 2.0 wt.\% CNT/2024Al, which could be due to the light atomicity of the CNT content, being too small to be detected by XRD. That is, the amount of CNTs of $<2.0 \mathrm{wt} . \%$ after the formation of some $\mathrm{Al}_{4} \mathrm{C}_{3}$ phase was below the detection limit of XRD. A close examination on the two XRD patterns in Fig.7.5 revealed broadening and lower intensities for the 2.0 wt.\% CNT/2024Al. This was attributed to the deformation, grain refinement and straining introduced by the CNTs. In fact, the effects of grain size and strain on the peak broadening could be distinguished on the basis of the Williamson-Hall approach [139],

$$
B \cos \theta=\frac{0.9 \lambda}{d_{g}}+2 \varepsilon \sin \theta
$$

where $B$ is the diffraction peak width at half-maximum intensity, $2 \theta$ is the Bragg diffraction angle, $\lambda$ is the wavelength of the radiation used, $d_{\mathrm{g}}$ is the average grain size, and $\varepsilon$ is the average internal strain. It is clear from Equ.(7.1) that the increase of the bandwidth of the peaks for the composite (Fig.7.5) was due to further straining and grain size reduction expected from CNTs. Peaks of the composite were also slightly delayed or shifted towards higher angles due to the impact of micro-strain on the distortion of the Al lattice because of the presence of CNTs.

\subsubsection{Lattice parameter of the alloy and the composite}

The lattice parameter $a_{o}$ was calculated in both the alloy and composite via Cohen's method [140] based on the least square refinement of the peak positions. According to the Bragg law,

$$
\lambda=2 d \sin \theta
$$


where $\lambda$ is the wavelength of the incident wave ( $\lambda=1.540598{ }^{\circ}$ in the current study), $d$ is the interplanar spacing and $2 \theta$ is the diffraction angle. By rearranging Equ.(7.2) one can write,

$$
\ln \left(\sin ^{2} \theta\right)=\ln \left(\frac{\lambda^{2}}{4}\right)-2 \ln d
$$

Differentiating Equ.(7.3) leads to,

$$
\frac{\Delta \sin ^{2} \theta}{\sin ^{2} \theta}=-2 \frac{\Delta d}{d},
$$

where $\frac{\Delta d}{d}$ is known as the systematic error, and is assumed to be in the form of,

$$
\frac{\Delta d}{d}=K \cos ^{2} \theta
$$

where $K$ is a constant. Substituting Equ.(7.5) into Equ.(7.4),

$$
\begin{gathered}
\frac{\Delta \sin ^{2} \theta}{\sin ^{2} \theta}=-2 K \cos ^{2} \theta, \\
\text { or } \Delta \sin ^{2} \theta=-2 K \cos ^{2} \theta \sin ^{2} \theta=D \sin ^{2} 2 \theta,
\end{gathered}
$$

with $D(=-\mathrm{K} / 2)$ is known as the drift constant, specific for every diffraction pattern [140].

In the Cohen's method $\Delta \sin ^{2} \theta$ is considered as,

$$
\Delta \sin ^{2} \theta=\sin ^{2} \theta-\sin ^{2} \theta_{0}
$$

where $\sin ^{2} \theta_{0}$ could be given by [44],

$$
\sin ^{2} \theta_{0}=\frac{\lambda^{2}}{4 a_{0}^{2}}\left(h^{2}+k^{2}+l^{2}\right)
$$

Considering Equ.(7.7) and (7.8), the new form of Equ.(7.6) becomes,

$$
\sin ^{2} \theta-\frac{\lambda^{2}}{4 a_{0}^{2}}\left(h^{2}+k^{2}+l^{2}\right)=D \sin ^{2} 2 \theta .
$$

Equ.(7.9) could then be generalized as, 


$$
\sin ^{2} \theta_{i}=A \alpha_{i}+D \delta_{i}
$$

where $A=\frac{\lambda^{2}}{4 a_{0}^{2}}, \alpha_{i}=h_{i}^{2}+k_{i}^{2}+l_{i}^{2}$, and $\delta_{i}=\sin ^{2} 2 \theta_{i}$

By combining Cohen's method with the least square approach, the optimum values of the coefficients $A$ and $D$ in Equ.(7.10) could be obtained by minimizing $f(A, D)$,

$$
f(A, D)=\sum_{i}\left(\sin ^{2} \theta_{i}-A \alpha_{i}-D \delta_{i}\right)^{2}
$$

Hence, the minimization condition was applied,

$$
\frac{\partial f(A, D)}{\partial A}=\frac{\partial f(A, D)}{\partial D}=0
$$

leading to a system of two equations,

$$
\left\{\begin{array}{l}
A \sum_{i} \alpha_{i}^{2}+D \sum_{i} \alpha_{i} \delta_{i}=\sum_{i} \sin ^{2} \theta_{i} \alpha_{i} \\
A \sum_{i} \alpha_{i} \delta_{i}+D \sum_{i} \delta_{i}^{2}=\sum_{i} \sin ^{2} \theta_{i} \delta_{i}
\end{array} .\right.
$$

Data needed to resolve the system of Equ.(7.13) was determined from the XRD patterns in Fig.7.5 and was summarized in Table 7.1. This system was solved twice for the base alloy and composite and using the obtained values of $A$ (i.e., $\left.A=\frac{\lambda^{2}}{4 a_{0}^{2}}\right), a_{o}$ was then calculated to be 4.059 $\stackrel{\circ}{A}$ for the $2024 \mathrm{Al}$ and $4.051 \stackrel{\circ}{A}$ for the $2.0 \mathrm{wt} . \% \mathrm{CNT} / 2024 \mathrm{Al}$ composite.

Table 7.1 Cohen method parameters for the determination of lattice constants

\begin{tabular}{c|ccccc}
\hline Peak No & h & k & l & $\boldsymbol{2 \theta} / \mathbf{2 0 2 4 A l}$ & $\mathbf{2 \theta} / \mathbf{2 . 0}$ wt.\% CNT/2024Al \\
\hline $\mathbf{1}$ & 1 & 1 & 1 & $38.4830^{\circ}$ & $38.5285^{\circ}$ \\
$\mathbf{2}$ & 2 & 0 & 0 & $44.7367^{\circ}$ & $44.7742^{\circ}$ \\
$\mathbf{3}$ & 2 & 2 & 0 & $65.1062^{\circ}$ & $65.1579^{\circ}$ \\
$\mathbf{4}$ & 3 & 1 & 1 & $78.2396^{\circ}$ & $78.3086^{\circ}$ \\
$\mathbf{5}$ & 2 & 2 & 2 & $82.4390^{\circ}$ & $82.5975^{\circ}$ \\
\hline
\end{tabular}


While the lattice parameter for the composite could not be found in the literature, the lattice parameter $a_{o}$ for the alloy was reported to be $4.06 \stackrel{\circ}{A}$ [141], confirming the validity of the result obtained above. The lattice parameter of 2024Al was also reported in [142] when studying the effect of dissolution of $\mathrm{Cu}$ and $\mathrm{Mg}$ atoms in the $\mathrm{Al}$ lattice, and they obtained a value of $4.062 \stackrel{\circ}{A}$. The slight difference from the present result (i.e., $4.059 \stackrel{\circ}{A}$ ) could be related to the different amounts (in wt.\%) of alloying elements between the currently-studied Al2024 (4.5 Cu, 1.5 Mg, 0.6 Mn) and the Al2024 in [142] (4.1 Cu, 1.9 Mg, 0.5 Si). The decrease of the lattice parameter in the composite justifies the delay of the peaks shown in Fig.7.5 due to the lattice shortening brought by the compressive micro-strains arising from the CNT addition. Qi and Wang [143] developed a model to link the lattice parameter to the volume fraction, size and shape of the nanoparticles in the metal matrix. A "shape factor" was introduced and was found to generate a $10 \%$ lattice change of the composite. The lattice parameter was also reported to decrease with increasing volume fraction of nanoparticles within the matrix.

\subsection{Crystallographic texture}

Fig.7.6 shows the $\{100\},\{110\}$ and $\{111\}$ pole figures determined via XRD for the 2024Al (Fig.7.6(a), (f), and (k)) and the 2.0 wt.\% CNT/2024Al (Fig.7.6(c), (h), and (m)). A respective comparison was made with the pole figures obtained via EBSD for the 2024Al (Fig.7.6(b), (g), and (i)) and for the 2.0 wt.\% CNT/2024Al (Fig.7.6(d), (i), and (n)). It is seen that the two methods showed good agreement for both materials in terms of the location of major components and maximum intensities. Hence, based on either method the results obtained for the alloy and the composite revealed a similar texture and standard texture components could be identified. 


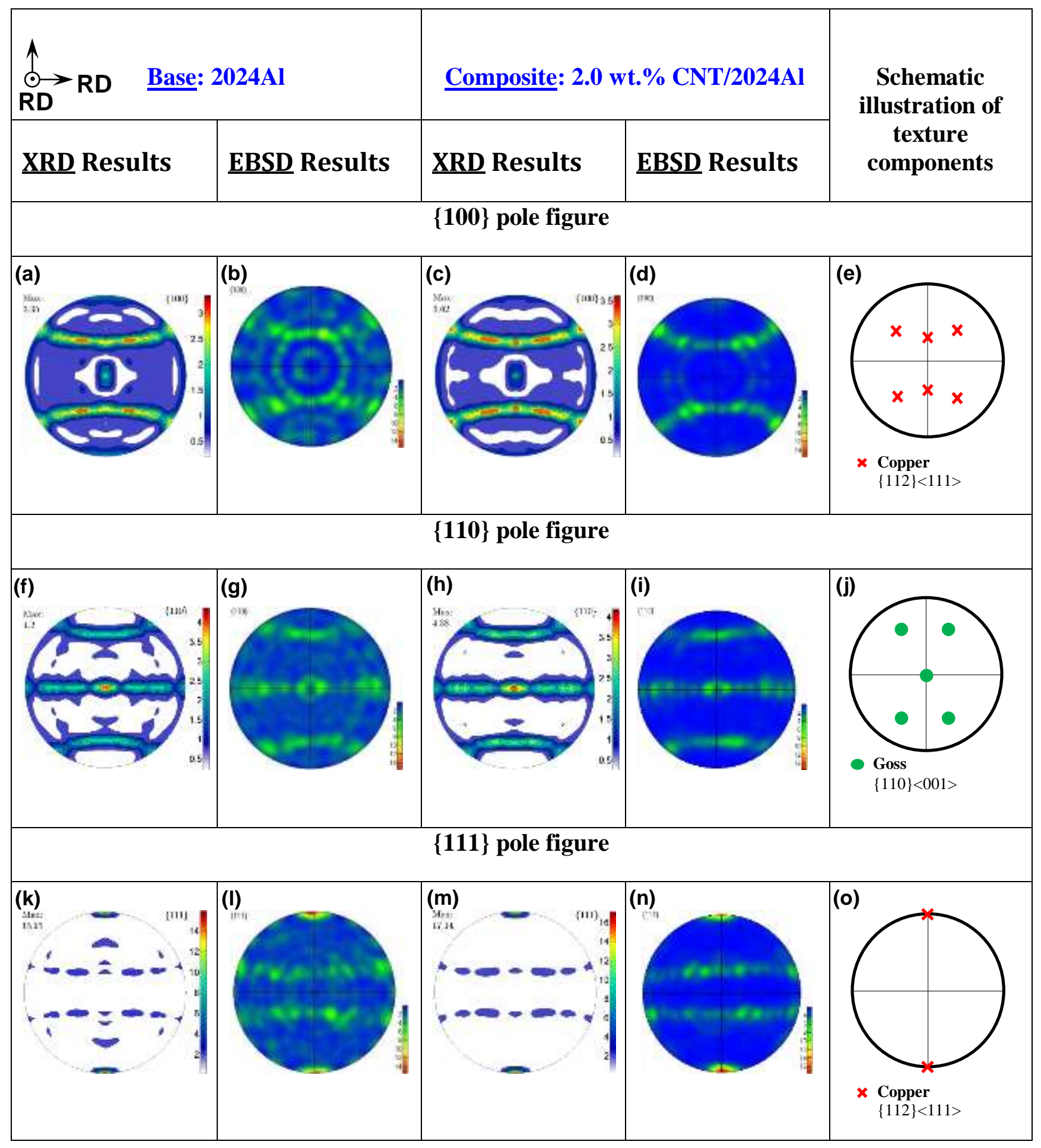

Fig.7.6 $\{100\},\{110\}$, and $\{111\}$ pole figures of the 2024Al alloy based on (a, f, k) XRD results and (b, g, l) EBSD results along with the $2.0 \mathrm{wt} . \% \mathrm{CNT} / 2024 \mathrm{Al}$ composite based on (c, h, m) XRD results and $(\mathrm{d}, \mathrm{i}, \mathrm{n})$ EBSD results, respectively, where ED stands for the extrusion direction, and $\mathrm{RD}$ denotes the radial direction, and $(\mathrm{e}, \mathrm{j}, \mathrm{o})$ indicate schematically the texture components. 
A closer look at the $\{100\}$ pole figure revealed the presence of $\{112\}<111>$ Copper texture [144] as illustrated in Fig.7.6(e). The formation of the Copper type texture could be linked to the deformation process where internal reaction stresses were induced within the matrix as well as external shear stresses brought by the impact of the extrusion dies [145]. Based on Fig.7.6(j), the $\{110\}$ pole figure allowed identifying the $\{110\}<001>$ Goss texture [146] known to occur as recrystallization texture in fcc materials. Copper texture was further seen from the $\{111\}$ pole figure where the location of the maximum intensities (Fig.7.6(o)) characterized the $\{112\}<111>$ Copper texture [144], corroborating the identification based on the $\{100\}$ pole figure. Texture strengthening for the composite compared to the base alloy was detected to a certain extent in all pole figures. For example, the intensity of Copper texture increased from $\sim 16.0$ multiples of random distribution (MRD) for the 2024Al to $17.1 \mathrm{MRD}$ for the $2.0 \mathrm{wt} . \% \mathrm{CNT} / 2024 \mathrm{Al}$ as seen from the $\{111\}$ pole figure. A less significant increase was observed for the Goss texture as seen from the $\{110\}$ pole figure, from $\sim 4.2$ for the base alloy to $\sim 4.4$ MRD for the composite. It should be noted that depending on the type of reinforcement and the initial texture, the strengthening of base alloy could lead to either stronger or weaker texture, and both scenarios have been reported. For instance, Kocks et al. [147] studied the texture evolution while comparing a 8090Al with two reinforced composites: (1) $15 \mathrm{vol} . \% \mathrm{SiC}$ whiskers/8090Al, and (2) 15 vol.\% SiC particles/8090Al. The intensity of texture became weaker in the SiC whisker reinforced composite, and stronger in the $\mathrm{SiC}$ particle reinforced composite, with respect to the base alloy. This observation underlines the impact of the type of reinforcement on the texture development, suggesting that the particles are not as effective as whiskers at repressing the texture [147]. Habibi et al. [148] reported the presence of both texture "hardening" and "softening" which they assumed strongly reliant on the CNT content and proved that the 
strengthening of a material could be achieved through the texture hardening. Conditions favoring stronger textures in the composite were discussed in [149] and were attributed to the presence of nanoparticle in the composite. Dislocation movement is strongly hindered by nanoparticles since the "geometrically necessary dislocations" at the matrix/CNT interface have to be stored to accommodate strain incompatibilities. Hence, nanoparticles facing a high dislocation density are "cut" by dislocations, so that local softening and strongly localized slip on only a few preferred slip systems will take place [149]. This will lead to less effective "constraints" for the deformation imposed by the surrounding grains, thus only the most favorably oriented slip systems will be active, resulting in a stronger preferred orientation [150].

\subsection{Compressive behavior}

Fig.7.7 shows the true stress-true strain compression curves of the base alloy and the composite obtained at different temperatures and strain rates. All the flow curves obtained at all temperatures and strain rates will be included in the appendix. In Fig.7.7(a) test temperatures were varied (i.e., $200,250,300,350$ and $400^{\circ} \mathrm{C}$ ) at a fixed strain rate of $0.001 \mathrm{~s}^{-1}$, whereas in Fig.7.7(b), tests were held at $300^{\circ} \mathrm{C}$ while varying the strain rates (i.e., 0.1, 0.01, and $0.001 \mathrm{~s}^{-1}$ ). A comparison between both plots affirms that the test temperature had a stronger effect on the deformation curves of both materials than the strain rate. Overall, the flow stress increased considerably as the true strain increased during the initial stages of deformation. Once the peak value was reached, a saturation level was attained regardless of the strain rate value. To quantify better the sensitivity of the alloy and the composite to both strain rate and temperature, Fig.7.8 was generated. CYS decreased with increasing temperatures and increased with strain rates. 

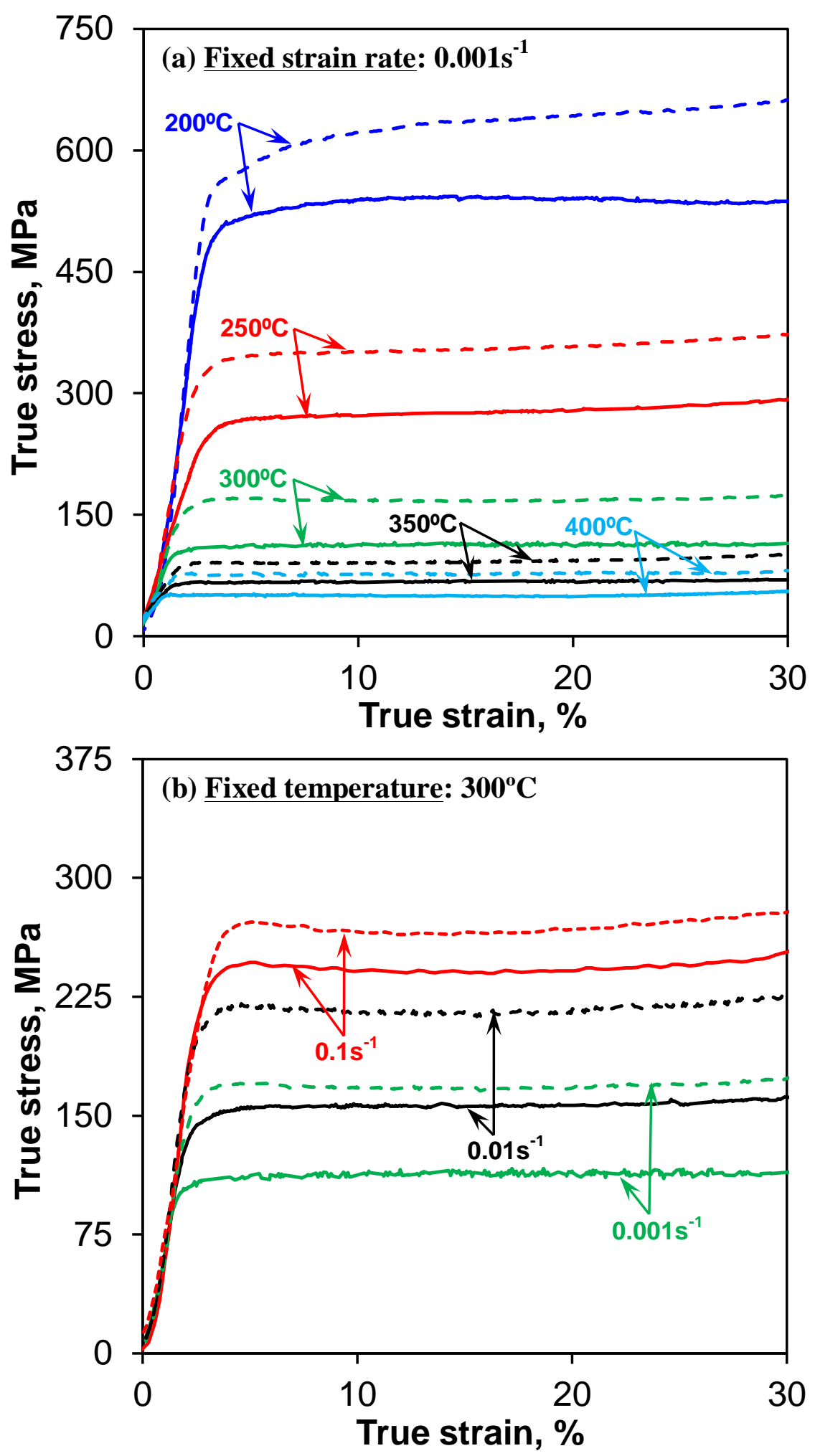

Fig.7.7 Compressive true stress-true strain curves of the 2024Al alloy (continuous lines) and the $2.0 \mathrm{wt} . \% \mathrm{CNT} / 2024 \mathrm{Al}$ composite (dashed lines): (a) at a strain rate of $0.001 \mathrm{~s}^{-1}$ and varying temperatures, and (b) at $300^{\circ} \mathrm{C}$ and varying strain rates. 
The dependence on the strain rate at different temperatures was less noticeable in the case of the composite. However, when comparing the behavior of both materials at a given strain rate and any temperature (Fig.7.8(a) and (b)), the strengthening behavior brought by CNT addition was clearly demonstrated through the enhancement of the CYS values for any chosen combination. This could be attributed to the Hall-Petch strengthening, also known as the GB strengthening where the material is strengthened by changing its average grain size due to the interaction between dislocations and GBs [151].

Fig.7.8(a) and (b) also showed that the evaluated CYS decreased with increasing temperatures at all strain rates for both materials. This could be attributed to the augmented thermal activation of the composite and the kinetic energy of the metal matrix which boosted the dislocation movements at higher temperatures. The significant improvement in the compressive properties of the composite at various temperatures and strain rates relative to the 2024Al base alloy could also be linked to the homogenous dispersion of the CNTs in the Al matrix as formerly seen in Fig.7.3(a).

Various factors related to the fabrication process might have also contributed to the enhancement of the flow behavior of the alloy and composite, mainly ball milling, which has resulted in a more refined microstructure characterized by improved high-temperature properties. In addition, the spontaneous reaction between $\mathrm{Al}$ and $\mathrm{O}_{2}$ when exposed to air during milling of the 2024Al powders, might lead to the formation of $\mathrm{Al}_{2} \mathrm{O}_{3}$ oxides. These particles might then be dispersed in the 2024Al matrix after extrusion, which could induce an increase in strength according to the Orowan strengthening mechanism $[152,153]$. 
Various mechanisms that played a role in the enhancement of the reinforced composite will be discussed in this section.
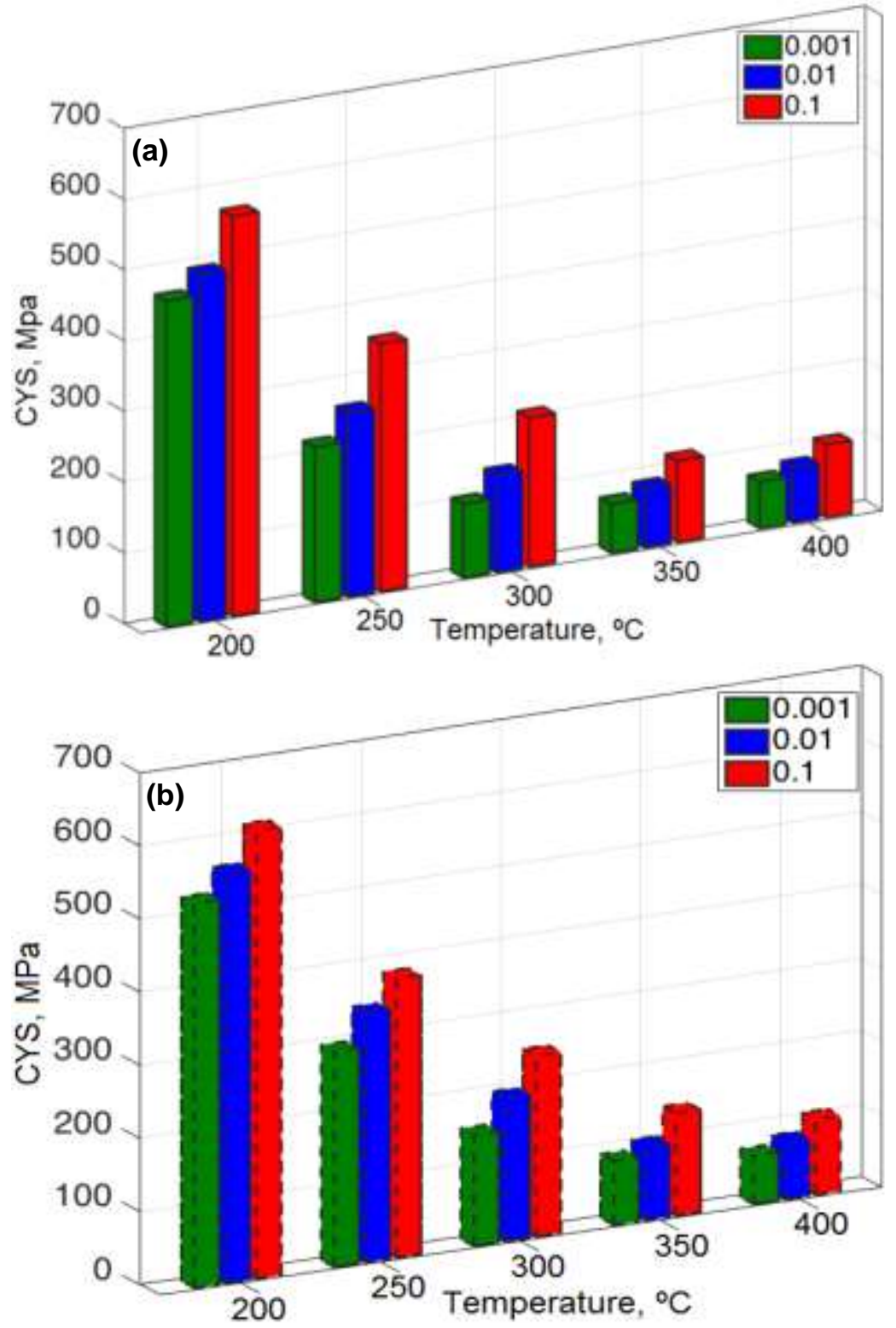

Fig.7.8 Change of compressive yield strength (CYS) with deformation temperature and strain rate of (a) the 2024Al alloy, and (b) the $2.0 \mathrm{wt}$ \% CNT/2024Al composite. Strain rates are expressed in $\mathrm{s}^{-1}$. 


\subsubsection{Grain refinement and grain boundary strengthening}

As previously seen in Fig.7.4, a clear grain refinement for the CNT reinforced 2024Al composite was attained. In fact, the impact of CNT addition on the grain refinement was well documented in the literature for MMCs. For instance, Suarez et al. [137] measured the mean grain size of a pure Ni, 1.0 wt.\%, 2.0 wt.\%, 3.0 wt.\% and 5.0 wt.\% MWCNT/Ni reinforced composites, and reported values of $47.6,22.4,4.9,4.9$, and $5.3 \mu \mathrm{m}$, respectively. Clearly, the grain size of the matrix was able to be refined once CNTs were added. The addition of CNTs up to 2.0 wt.\% could decrease the grain size significantly, beyond which no further grain refinement was achieved. Thus, an "empirical reinforcement limit" at 2.0 wt.\% MWCNT was specified [137]. Liu et al. [95] indicated that singly dispersed CNTs in the composite tended to be dispersed along GBs resulting in a much finer grain size. This could be linked to the results presented in Fig.7.3(a), where TEM observations revealed singly dispersed CNTs.

Hall-Petch strengthening (i.e., GB strengthening) is directly linked to the CYS enhancement when the composite is strengthened by refining its average grain size [154]. The motion of dislocations during deformation is impeded by GBs due to the misorientation between neighboring grains. The smaller the grain size is, the more GBs are present, leading to a more difficult dislocation movement and thus a higher flow stress. This is due to the fact that GBs act as "pinning points" prohibiting the dislocation movement and the eventual multiplication of dislocations. This leads to a "pile up" of dislocation clusters being unable to move past a boundary. Dislocations could be rearranged into low-energy structures (i.e., substructures) characterized by their low angle grain boundaries (LAGBs). In this context, Fig.7.9(a) displays 
the grain boundary mapping of the $2.0 \mathrm{wt} . \% \mathrm{CNT} / 2024 \mathrm{Al}$ along with the misorientation angle histogram (Fig.7.9(b)). A bi-modal distribution is observed where a moderately important portion of the microstructure was characterized by LAGBs (i.e., angles of $<10^{\circ}$ ) which confirms the previously described kinetics of grain refinement.

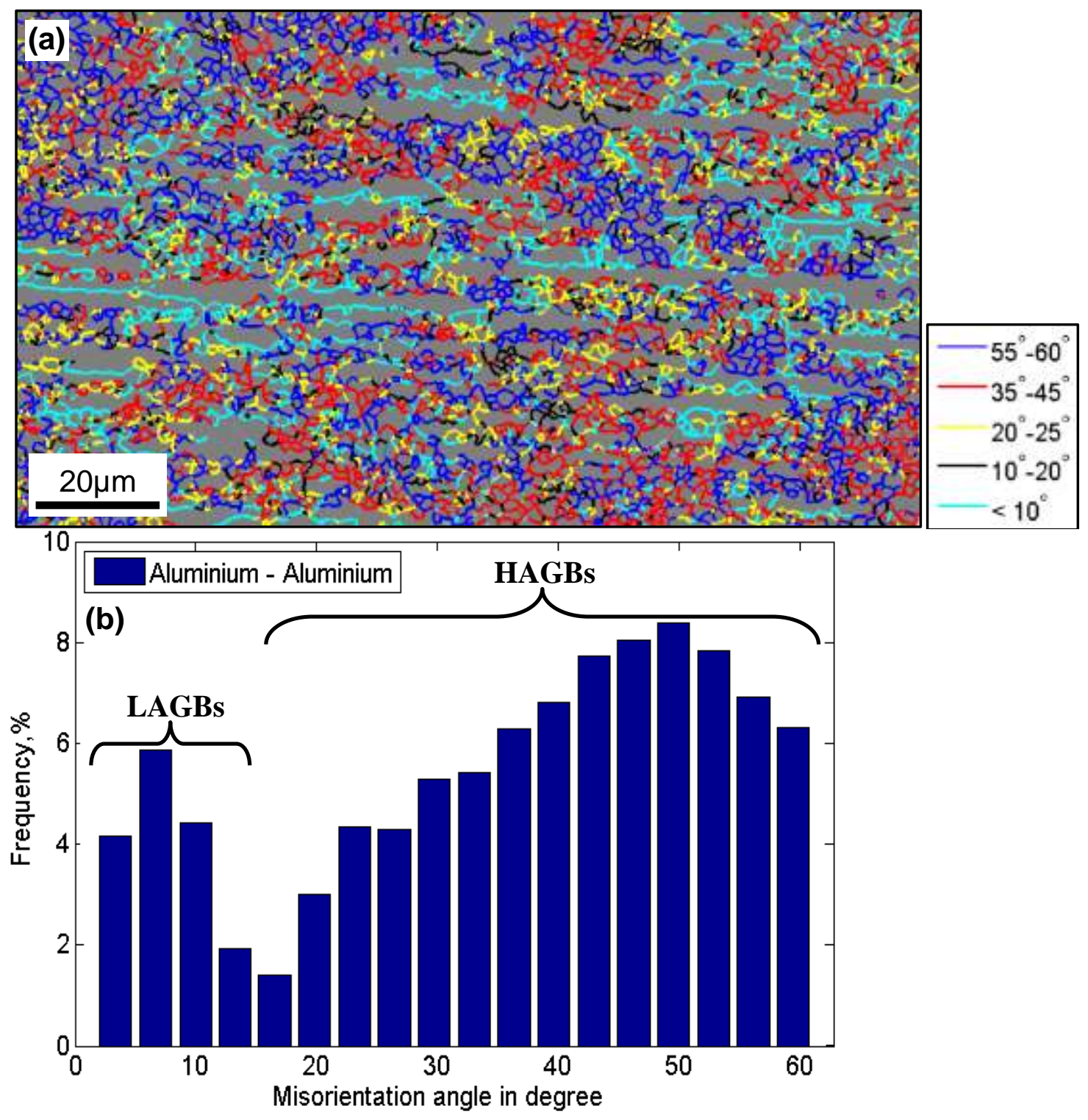

Fig.7.9 (a) Grain boundary mapping along with (b) the misorientation angle histogram for the 2.0 wt.\% CNT/2024Al composite. 
The presence of LAGBs would be associated with the formation of subgrains/substructures during ball milling. A majority of high angle grain boundaries (HAGBs) shown in Fig.7.9 would be linked to the happening of recrystallization to be discussed below.

\subsubsection{Recrystallization and built-up of substructures}

Recrystallization is directly linked to the strengthening behavior since it is related to the evolution of the grain microstructure. Various possible scenarios could lead to the occurrence of recrystallization, where the most common one is the formation and migration of HAGBs. This is in good agreement with the results observed in Fig.7.9 about the dominance of misorientation angles $>25^{\circ}$. In other words, dislocation rearrangements leading to the formation of low dislocation density regions (associated with HAGBs) are capable of fast migration over the strained matrix.

Due to the existence of subgrains in the $2.0 \mathrm{wt} . \% \mathrm{CNT} / 2024 \mathrm{Al}$ composite as revealed by the LAGBs in Fig.7.9, the nucleation of recrystallization by means of subgrain coalescence could also be another scenario. Earlier studies by Sandstrom et al. [155] introduced subgrain coalescence as the subgrain growth and the disappearance of sub-boundaries which lead to the decrease of the stored energy and the change of orientations between coalesced and neighboring subgrains. This promotes the formation of HAGBs having the ability to migrate fast and to form the recrystallization nucleus. The happening of recrystallization by the migration of LAGBs (i.e., sub-boundaries) has been well documented in the literature dating back to the Cahn-Cottrell model [156]. 


\subsubsection{Zener drag effect}

Second-phase particles (i.e., nano-sized CNTs and Cu-containing particles) played an important role in controlling grain size and texture, in stimulating the nucleation of recrystallization and in pinning boundaries. To move a boundary past a particle, a force has to be exerted which is related to the size of the particle and the energy of the boundary [157]. In a random distribution of particles which are assumed under a spherical shape, it is possible to express the pinning force $P_{z}$ exerted on the boundary could be given by [158],

$$
P_{z}=\frac{3 \gamma V_{p}}{2 r},
$$

where $\gamma$ is the grain boundary energy, $V_{p}$ is the particle volume fraction and $r$ is the particle radius. Based on Equ.(7.14), the presence of small and very closely spaced particles play the role of pinning sites on the grain boundaries [159]. In the case of the studied 2.0 wt.\% CNT/2024Al, Equ.(7.14) would have to be adjusted for the tube shape of the CNTs by considering a length component in the equation. Overall, independently from the particle shape, the stagnation from the Zener drag effect happened due to the motion restriction of GBs mainly by the CNTs which act as the pinning force or sometimes called "frictional forces" to the moving GBs, prohibiting the grain growth and therefore leading to the grain refinement as observed in Fig.7.4(b).

\subsubsection{Load transfer, thermal mismatch and Orowan looping}

Load transfer, generation of dislocations by thermal mismatch, and Orowan looping were discussed in the literature as major mechanisms for the enhancement of the mechanical 
properties of CNT/Al reinforced composites $[135,136,160]$. These mechanisms were generally thought to occur simultaneously where the strength of the composite is the sum of each improvement brought from each of the afore-mentioned mechanisms, plus the inherent strength of the matrix itself. At the atomic level, a strong cohesion between the matrix and CNTs can be attained due to the nano-size of the reinforcement, leading to a direct bond between the matrix and reinforcement (Fig.7.3(b)). Hence, depending on their volume fraction, CNTs would carry a certain portion of the load (i.e., load transfer) between matrix and reinforcement. The basis of the load transfer theory was initially introduced by Kelly and Tyson [161]. They stated that the applied stresses could be transferred to the reinforcements (e.g., CNTs) through interfacial shear stresses emanating from the matrix. In the case of the studied $2.0 \mathrm{wt} . \% \mathrm{CNT} / 2024 \mathrm{Al}$, this could be justified by the formation of the $\mathrm{Al}_{4} \mathrm{C}_{3}$ phase at the interface. Similarly, Kwon et al. [162] and Bakshi and Agarwal [24] reported that the formation of $\mathrm{Al}_{4} \mathrm{C}_{3}$ is necessary for an effective load transfer between the matrix and CNTs.

The improved strength of the composite associated with the generation of dislocations by thermal mismatch is produced from the "mismatch" in the coefficients of thermal expansions (CTEs) of the CNTs $\left(\sim 1 \times 10^{-6}{ }^{\circ} \mathrm{C}^{-1}[44]\right)$ and the $2024 \mathrm{Al}$ matrix $\left(\sim 22.9 \times 10^{-6}{ }^{\circ} \mathrm{C}^{-1}[44]\right)$, in the cooling phase of processing. This engenders the formation of dislocations, and with increasing their density in the matrix, the composite is strengthened. This improvement was attributed by George et al. [160] to the "prismatic punching" of dislocations at the interface, stimulating the work hardening of the matrix. 
1. The addition of $2.0 \mathrm{wt} . \% \mathrm{CNTs}$ in a $2024 \mathrm{Al}$ alloy led to fine grain sizes along with some $\mathrm{Cu}-$ containing second phase particles. TEM examinations revealed singly dispersed CNTs in the matrix, although the shortening of CNTs was observed to occur due to ball milling.

2. The presence of $\mathrm{Al}_{4} \mathrm{C}_{3}$ phase, being either directly attached to CNTs or in the matrix in the neighborhood of CNTs, suggested the occurrence of reactions between the Al matrix and CNTs during the composite synthesis.

3. The X-ray diffraction peaks of the CNT reinforced composite were observed to be broadened due to the grain size reduction and compressive micro-strains brought by the CNT addition. The presence of compressive micro-strains also led to the lattice shortening.

4. The deformation resistance of the composite at both RT and HT was effectively enhanced owing to the addition of CNTs. This was mainly associated to the Hall-Petch strengthening, and composite strengthening including load transfer, thermal mismatch and Orowan looping. 


\section{CHAPTER 8}

\section{HOT DEFORMATION BEHAVIOR OF 2024AI BASE ALLOY AND 2.0 WT.\% CNT REINFORCED 2024AI COMPOSITE}

\subsection{Introduction}

The optimized processing window for a given material at high temperatures is determined based on its workability parameters mainly, activation energy, efficiency of power dissipation, instability criteria, and existence of favorable microstructural mechanisms. Constitutive equations were employed to evaluate material characteristics and correlate them to the hot deformation behavior.

\subsection{Constitutive modeling}

Relationship between strain rate, deformation temperature, and flow stress was extensively used in literature through the Arrhenius equations, after being initially proposed by [163] as follows:

$$
\dot{\varepsilon}=A[\sinh (\alpha \sigma)]^{n} \exp \left(-\frac{Q}{R T}\right)
$$

where $n$ and $A$ are material constants, $\alpha$ is a stress multiplier, $\sigma$ is the flow stress (MPa), $Q$ is the activation energy for hot deformation $(\mathrm{kJ} / \mathrm{mol}), R$ is the universal gas constant $(8.314 \mathrm{~J} / \mathrm{mol}-\mathrm{K})$, and $T$ is the deformation temperature $(\mathrm{K})$. For high and low stress levels, the relationship between the flow stress and strain rate could be described by [164]:

$$
\dot{\varepsilon}=A_{1} \sigma^{n_{1}} \exp \left(-\frac{Q}{R T}\right)
$$




$$
\dot{\varepsilon}=A_{2} \exp (\beta \sigma) \exp \left(-\frac{Q}{R T}\right),
$$

where $A_{1}$ and $A_{2}$ are the material constants. Eqs.(8.4) and (8.5) are obtained by taking the natural logarithm on both sides of Eqs.(8.2) and (8.3) such as:

$$
\begin{gathered}
\ln \dot{\varepsilon}=\ln A_{1}+n_{1} \ln \sigma-\frac{Q}{R T}, \\
\ln \dot{\varepsilon}=\ln A_{2}+\beta \sigma-\frac{Q}{R T} .
\end{gathered}
$$

The term $\sigma$ is substituted in the current study by the CYS values. Data obtained at the different test temperatures (i.e., $200,250,300,350$, and $400^{\circ} \mathrm{C}$ ) were taken into account to compute the activation energy for the alloy and the composite.

Fig.8.1(a) shows the plot of $\ln \dot{\varepsilon}$ vs. $\ln \sigma$ for both alloy and composite. A linear relationship was noted for both cases having the slope $n_{1}$ as introduced in Equ.(8.4). Fig.8.1(b) displays the relation between $\ln \dot{\varepsilon}$ and $\sigma$ for both materials. A linear trend was also depicted and characterized by the slope $\beta$ previously introduced in Equ.(8.5). Substituting the values of CYS for alloy and composite at the corresponding strain rates and temperatures into Equ.(8.4) and (8.5), the values of $n_{l}$ and $\beta$ could be obtained while accounting for a linear regression. Average slope values were taken to calculate $n_{l}$ and $\beta$. The stress multiplier $\alpha$ could also be estimated as $\alpha=\beta / n_{l}$. The obtained values of $n_{1}, \beta$, and $\alpha$ for the alloy and composite have been summarized in Table 8.1. Back to Equ.(8.1), and taking its the natural logarithm on both sides, Equ.(8.6) could be expressed as,

$$
\ln \dot{\varepsilon}=\ln A+n \ln [\sinh (\alpha \sigma)]-\frac{Q}{R T} .
$$


Fig.8.1(c) shows the evolution of $\ln \dot{\varepsilon}$ vs. $\ln (\sinh (\alpha \sigma))$ where a linear trend was also depicted for the $2024 \mathrm{Al}$ and the $2.0 \mathrm{wt} . \% \mathrm{CNT} / 2024 \mathrm{Al}$, and characterized by the average slope $n$. Obtained values of $n$ for alloy and composite were also included in Table8.1.
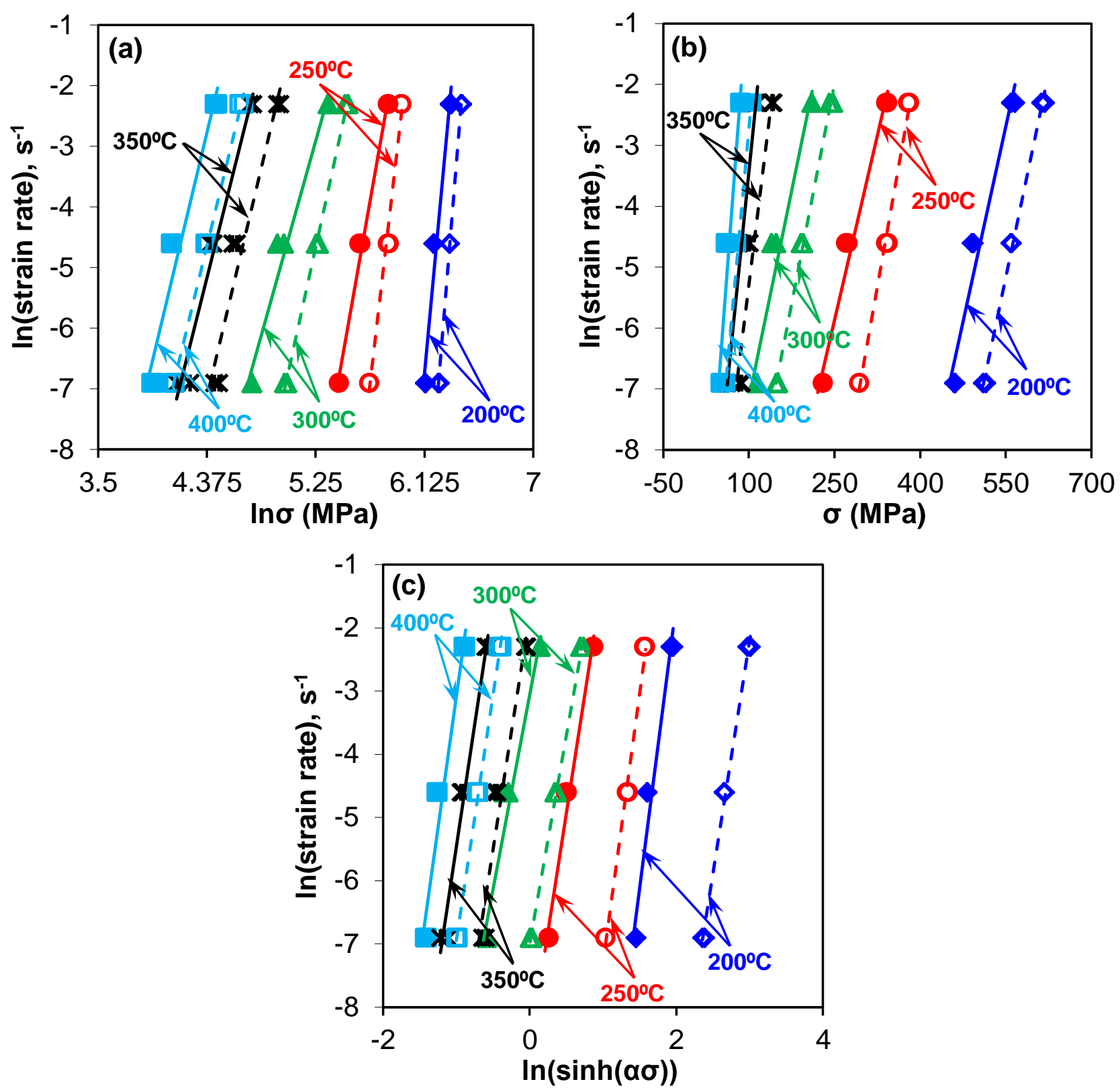

Fig.8.1The relationship of CYS, strain rate and temperature of the base 2024Al alloy (continuous lines) and the $2.0 \mathrm{wt} . \% \mathrm{CNT} / 2024 \mathrm{Al}$ (dashed lines): (a) $\ln (\dot{\varepsilon})$ versus $\ln (\sigma)$, (b) $\ln (\dot{\varepsilon})$ versus $\sigma$, and (c) $\ln (\dot{\varepsilon})$ versus $\ln [\sinh (\alpha \sigma)]$. 
Table 8.1 Main parameters of the hot deformed 2024Al alloy and the 2.0 wt.\% CNT/2024Al composite evaluated from the constitutive equations.

\begin{tabular}{ccccccc}
\hline & $\boldsymbol{n}_{\boldsymbol{1}}$ & $\boldsymbol{\beta}$ & $\boldsymbol{\alpha}$ & $\boldsymbol{n}$ & $\boldsymbol{S}$ & $\boldsymbol{Q}, \mathbf{k J} / \mathbf{m o l}$ \\
\hline 2024Al & 13.84 & $64.88 \times 10^{-3}$ & $4.68 \times 10^{-3}$ & 7.15 & 4.61 & $\mathbf{2 7 4 . 5}$ \\
$\begin{array}{c}\text { 2.0 wt.\% } \\
\text { CNT/2024Al }\end{array}$ & 11.53 & $69.04 \times 10^{-3}$ & $5.98 \times 10^{-3}$ & 7.09 & 5.46 & $\mathbf{3 2 2 . 3}$ \\
\hline
\end{tabular}

\subsection{Activation energy of hot deformation}

The activation energy $Q$, is generally related to the thermodynamic mechanism of dislocation movement and can reveal the workability of materials [165]. It is in fact a measure of the minimum energy required to initiate dislocation movement by diffusion, and is considered to be an important physical parameter indicating the degree of difficulty to deform a material under specific deformation conditions [166]. Activation energy could be determined after taking partial differential of Equ.(8.6), the following could then be obtained,

$$
Q=R\left[\frac{\partial \ln \dot{\varepsilon}}{\partial \ln [\sinh (\alpha \sigma)]}\right]_{T} \cdot\left[\frac{\partial \ln [\sinh (\alpha \sigma)]}{\partial(1 / T)}\right]_{\dot{\varepsilon}}=R n S
$$

The newly introduced parameter $S$ in Equ.(8.7), is the average slope of the $\ln [\sinh (\alpha \sigma)]-1 / T$ plot at various strain rates (Fig.8.2(a) and (b) for the alloy and the composite respectively). The obtained values of $S$ for the 2024Al and the $2.0 \mathrm{wt} . \%$ CNT 2024Al composite are also included in Table 8.1. Activation energy $Q$ could thus be calculated based on Equ.(8.7) and was obtained to be $274.5 \mathrm{~kJ} / \mathrm{mol}$ for the alloy and $322.3 \mathrm{~kJ} / \mathrm{mol}$ for the composite (Table 8.1 ) which are both higher than that for self-diffusion in pure aluminum [167] as expected, due to the impact of 
alloying elements and the CNT addition on the thermos-mechanical properties of alloy and composite. Different values have been reported in literature for the activation energy of $\mathrm{Al}$ alloys. For instance Wang et al. [168] stated that for the AA7050 alloy, it could go as high as 470.2 $\mathrm{kJ} / \mathrm{mol}$ at $603 \mathrm{~K}$ and a strain rate of $0.001 \mathrm{~s}^{-1}$, and as low as $82.8 \mathrm{~kJ} / \mathrm{mol}$ at $693 \mathrm{~K}$ and $10 \mathrm{~s}^{-1}$. A similar temperature range as the current study $\left(200-400^{\circ} \mathrm{C}\right)$ was considered in [169] and activation energy of $282.6 \mathrm{~kJ} / \mathrm{mol}$ was reported for the $\mathrm{Al}-\mathrm{Si}$ alloy, which is comparable to the obtained value for the 2024Al alloy $(274.5 \mathrm{~kJ} / \mathrm{mol})$. The alloy reported in [169] was then modified by adding $\mathrm{V}$ and $\mathrm{Zr}$ leading to an activation energy of $315.2 \mathrm{~kJ} / \mathrm{mol}$. The previous emphasizes the impact of the alloying elements, analogue to the CNT additions in the current study, where higher activation energy was obtained for the composite $(322.3 \mathrm{~kJ} / \mathrm{mol})$. This could be linked to the fact that the $2.0 \mathrm{wt} . \% \mathrm{CNT} / 2024 \mathrm{Al}$ was treated under longer homogenization times leading to a higher strength, and hence a more important lattice resistance. Compared to the base alloy, due to the presence of CNTs, dislocations were pinned by the nanoparticles and encountered friction drags. Both thermal energy and external stresses required for dislocation motion increased. The presence of finer particles is therefore crucial for the increase of activation energy. Malas et al. [167] also compared the activation energy of (1) wrought 2024Al, (2) powder metallurgy 2024Al, and (3) PM 2024Al with $\mathrm{SiC}$ whiskers $\left(\mathrm{SiC}_{\mathrm{W}}\right)$. The flowing intervals for $Q$ were reported for the enumerated materials: (1) [90-200] kJ/mol, (2) [167-418] kJ/mol, and (3) [377-1047] kJ/mol. First analogy that could be made with the results of the current study is that the obtained value for the $2024 \mathrm{Al}(Q=274.5 \mathrm{~kJ} / \mathrm{mol})$ falls within the PM $2024 \mathrm{Al}$ intervals (i.e., ball milling in the current study). The second observation is about the tremendous increase in the values of $Q$ due to $\mathrm{SiC}_{\mathrm{W}}$ reinforcement, although greater values were observed interval (3) above) compared with the current $2.0 \mathrm{wt} . \% \mathrm{CNT} / 2024 \mathrm{Al}$ composite $(Q=322.3 \mathrm{~kJ} / \mathrm{mol})$. The 
higher values obtained for the $\mathrm{SiC}_{\mathrm{W}}$ were expected since it involves rigid ceramic phases in a plastically deforming 2024Al matrix.
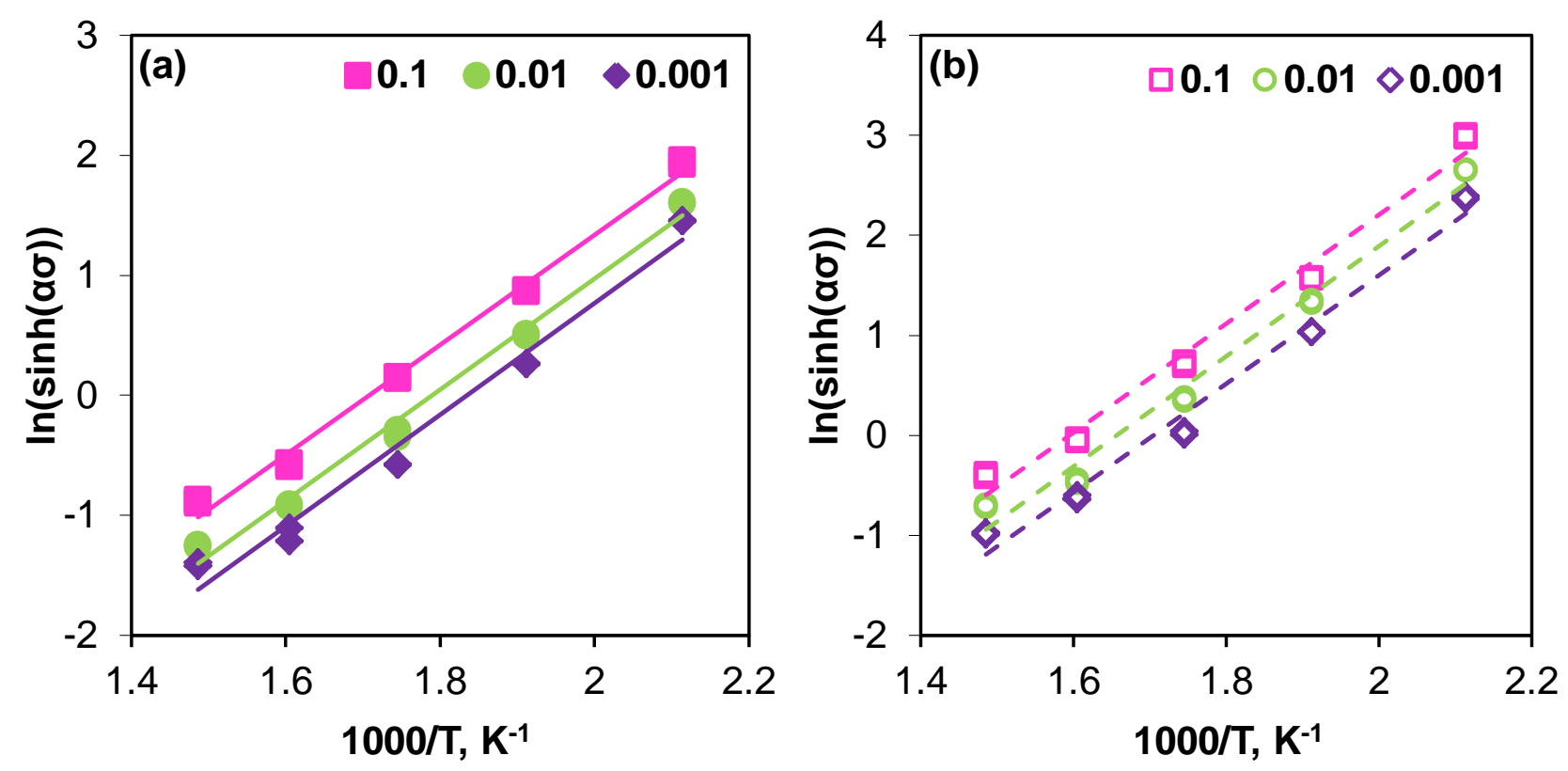

Fig.8.2 Relationship of CYS, strain rate and temperature in terms of $\ln [\sinh (\alpha \sigma)]$ versus $1000 / T$ (a) for the $2024 \mathrm{Al}$ alloy, and (b) for the $2.0 \mathrm{wt} . \% \mathrm{CNT} / 2024 \mathrm{Al}$ composite. Strain rates are expressed in $\mathrm{s}^{-1}$.

\subsection{Basis of processing maps}

Processing maps represent an effective alternative for determination of optimized hot workability regions of materials. The concept of processing maps relies on the dynamic material model (DMM) [169-171], according to which the workpiece dissipates power during hot deformation and the constitutive response of the material at a given temperature depends essentially on strain rate and to a lesser extent on strain. The total power per unit volume $P$ absorbed by the 
workpiece during plastic flow may be expressed as a sum of two complementary functions [172] such as,

$$
P=G+J=\int_{0}^{\dot{\varepsilon}} \sigma d \dot{\varepsilon}+\int_{0}^{\sigma} \dot{\varepsilon} d \sigma
$$

where $G$ represents the power dissipated by plastic deformation, most of which is converted into heat, and $J$ represents the dissipation through microstructure evolution (e.g., dynamic recovery, dynamic recrystallization, superplastic flow, phase transformation, crack propagation). At any given temperature and strain, the partitioning of power between $J$ and $G$ is given by [166],

$$
\frac{\partial J}{\partial G}=\frac{\partial \ln \sigma}{\partial \ln \dot{\varepsilon}}=m
$$

where $m$ is the strain rate sensitivity of the material. Knowing the values of $m$, dissipation through the microstructure evolution $J$ may be normalized with respect to $J_{\max }$ to obtain a dimensionless parameter called the efficiency of power dissipation [170] such as,

$$
\eta=\frac{J}{J_{\max }}=\frac{2 m}{m+1}
$$

The variation of $\eta$ with temperature and strain rate reveals the features of power dissipation arising through microstructural changes in the workpiece, creating the power dissipation map. This map could be presented as contour plots of efficiency variation in the temperature-strain rate domain where different regions (i.e., corresponding to different $\eta$ values) signify different deformation mechanisms. A continuum instability criterion, obtained based on the extremum principles of irreversible thermodynamics, could also be provided [173],

$$
\xi(\dot{\varepsilon})=\frac{\partial \ln (m / m+1)}{\partial \ln \dot{\varepsilon}}+m<0
$$


Equ.(8.11) leads to obtaining the instability parameter $\xi(\dot{\varepsilon})$ which is used to mark out the flow instability regions, after being evaluated as function of temperature and strain rate. The instability map was obtained when $\xi(\dot{\varepsilon})<0$, representing the occurrence of flow instability. As stated above, with the values of $\eta$ and $\xi(\dot{\varepsilon})$ under different temperatures, strain rates and strains, processing maps can be established by superimposing the instability maps over the power dissipation maps. Conventional 2-D maps and 3-D processing maps will be analyzed in the coming section.

\subsection{Conventional 2-D processing maps}

Fig.8.3(a), (b) and (c) display the 2D processing maps of the 2024Al obtained at strain values of $0.1,0.3$, and 0.5 respectively. Equal strain values were chosen for the $2.0 \mathrm{wt} . \% \mathrm{CNT} / 2024 \mathrm{Al}$ as presented in Fig.8.4(a), (b), and (c). Colored areas in Fig.8.3 and 8.4 separated by contour lines designate the degree of efficiency (i.e., reflecting $\eta$ values calculated based on Equ.(8.10)). The limit of the highest efficiency region in each map was approximated by the dashed lines, i.e., marking the regions $R_{1}$ in Fig.8.3 and 8.4. The highlighted regions $R_{2}$, denoted the flow instability domains plotted based on Equ.(8.11). Peak efficiency values showed a slight variation from a map to the other for both materials. For instance, for the 2024Al, peak values of $\eta$ varied from $\sim 35 \%$ at $\varepsilon=0.1$, to $\sim 37 \%$ at $\varepsilon=0.3$, to $\sim 39 \%$ at $\varepsilon=0.5$. Similarly, a variation of the maximum $\eta$ values was reported in [170] for the $2024 \mathrm{Al}$ alloy, from $\sim 39 \%$ at $\varepsilon=0.1$, to $\sim 44 \%$ at $\varepsilon=0.3$, to $\sim 46 \%$ at $\varepsilon=0.5$. The larger $\eta$ values in [170] than to the current study could be related to the bigger temperature range (up to $500^{\circ} \mathrm{C}$ ), compared with the upper limit of $400^{\circ} \mathrm{C}$ in the 
present work. Efficiency was also reported in [171] to increase with increasing strain, which was observed for the composite in Fig.8.4 as well ( $\eta \sim 27 \%$ at $\varepsilon=0.1, \sim 29 \%$ at $\varepsilon=0.3$, and $\sim 30 \%$ at $\varepsilon=0.5)$.
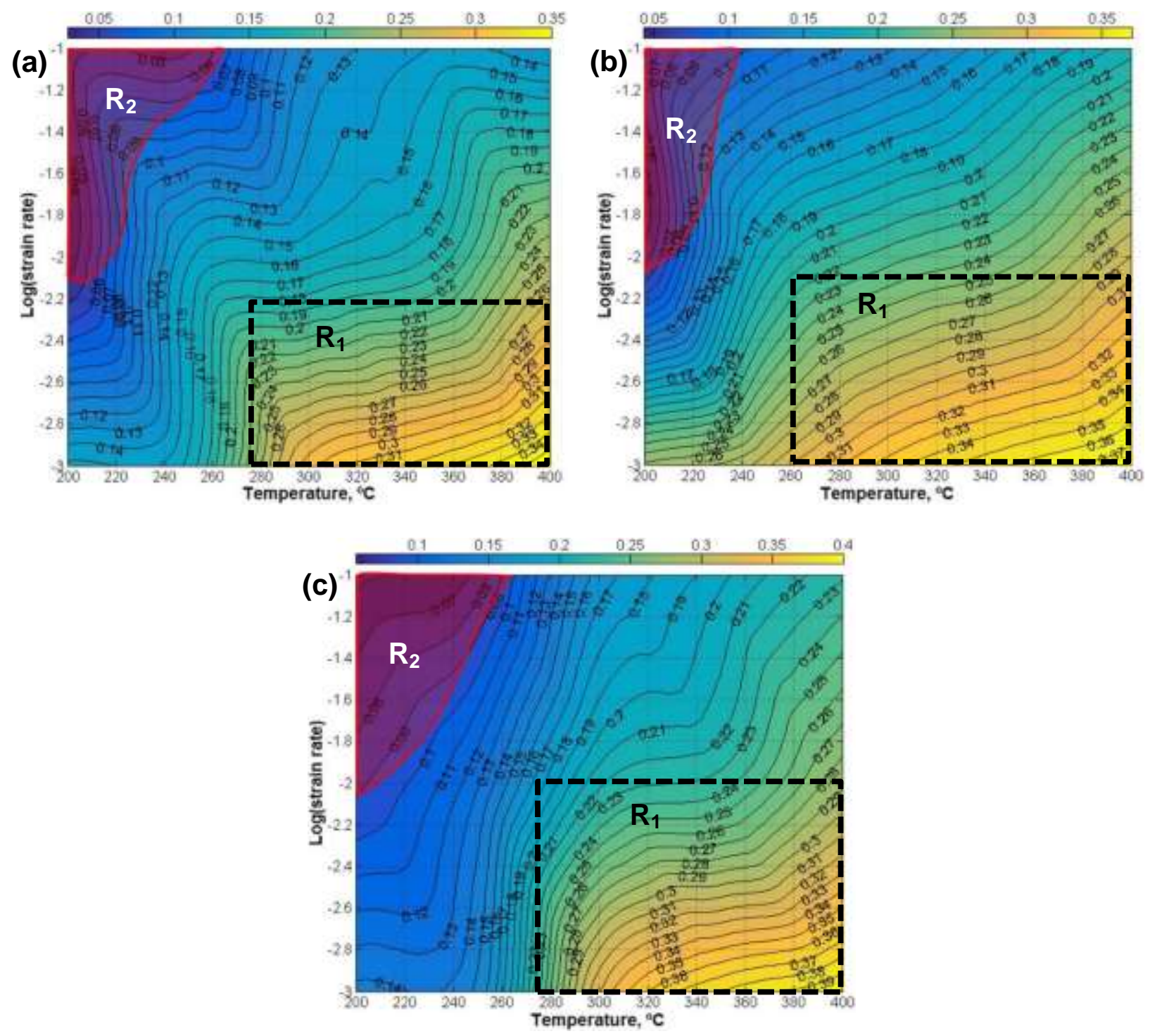

Fig.8.3 Processing maps of the 2024Al alloy for the true strain values of (a) $\varepsilon=0.1$, (b) $\varepsilon=0.3$, and (c) $\varepsilon=0.5$, respectively.

Based on Fg.8.3 and 8.4, $\mathrm{R}_{1}$, representing the stable domains where fairly high values of efficiency existed, coincided with the samples deformed at higher temperatures and lower strain 
rates. However, $\mathrm{R}_{2}$ corresponded to lower temperatures and higher strain rates. Limits of $\mathrm{R}_{1}$ were slightly variable with strain for both alloy and composite. For the 2024Al, it went from 280 $400^{\circ} \mathrm{C} / 0.001-0.006 \mathrm{~s}^{-1}$ at $\varepsilon=0.1$, to $260-400^{\circ} \mathrm{C} / 0.001-0.008 \mathrm{~s}^{-1}$ at $\varepsilon=0.3$, and $275-400^{\circ} \mathrm{C} / 0.001-$ $0.01 \mathrm{~s}^{-1}$ for $\varepsilon=0.5$. As for the composite in Fig.8.4, $\mathrm{R}_{1}$ also varied based on strain: 303$400^{\circ} \mathrm{C} / 0.001-0.01 \mathrm{~s}^{-1}$ at $\varepsilon=0.1,280-400^{\circ} \mathrm{C} / 0.001-0.006 \mathrm{~s}^{-1}$ at $\varepsilon=0.3$, and $285-400^{\circ} \mathrm{C} / 0.001-$ $0.009 \mathrm{~s}^{-1}$ at $\varepsilon=0.5$.
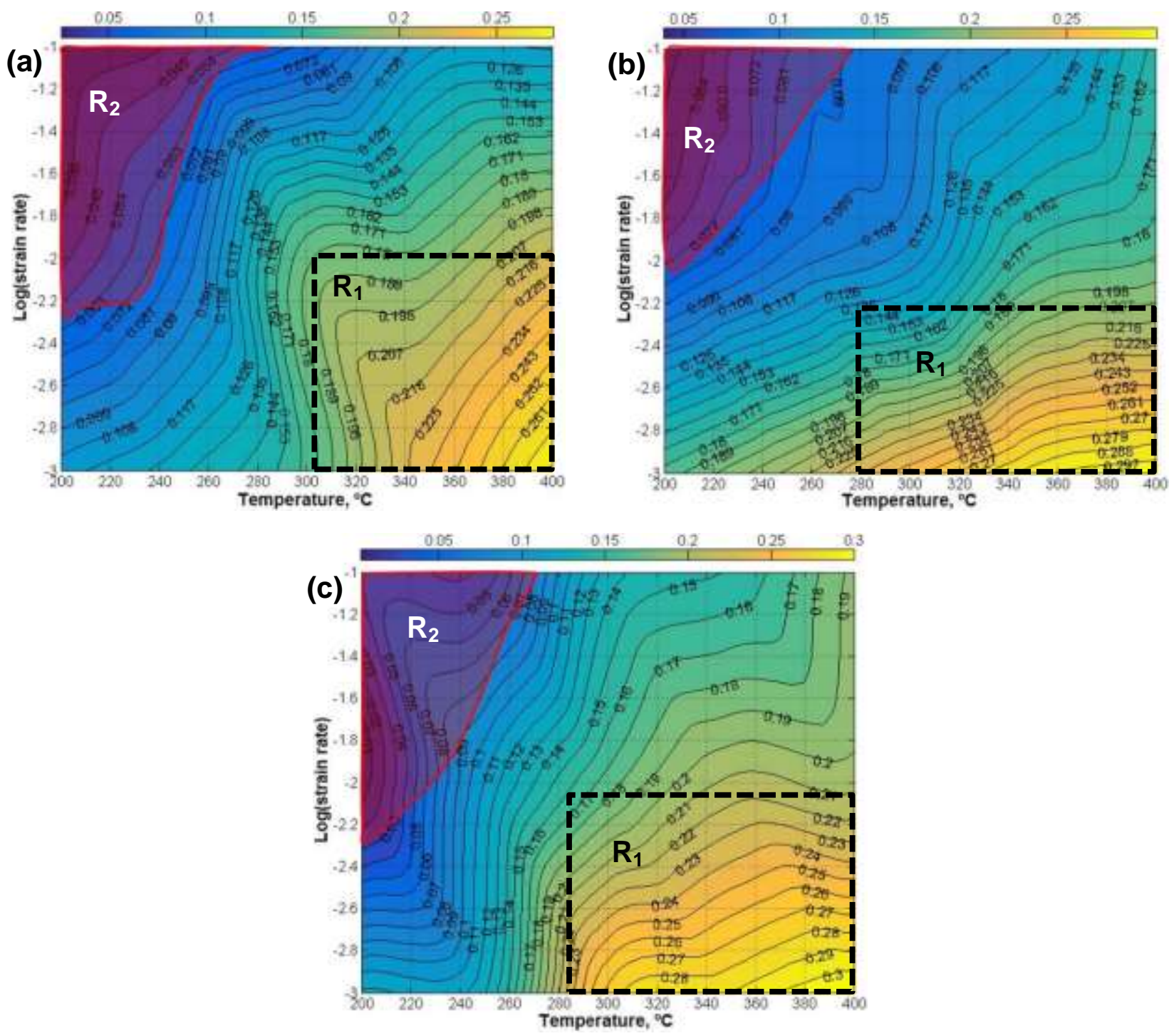

Fig.8.4 Processing maps of the 2.0 wt. $\% \mathrm{CNT} / 2024 \mathrm{Al}$ composite for the true strain values of (a) $\varepsilon=0.1$, (b) $\varepsilon=0.3$, and (c) $\varepsilon=0.5$, respectively. 
Instability regions $\mathrm{R}_{2}$, although observed to persist around same locations, were also perceived to vary moderately with strain for both materials. By comparing the extent of each of the regions between alloy and composite, the "safe" (i.e., stable) window was observed to relatively shrink for the composite. The previous was expected since structural complexity introduced by CNT addition led to the restriction of the working window, demanding greater process control. Likewise, Malas et al. [167] studied the difference of the optimum processing windows between a P/M-2024Al and a P/M-2024Al reinforced with 20 v/o $\mathrm{SiC}_{\mathrm{w}}$. They reported that the safe processing for the composite occurred over a narrower range of temperature and strain rate, which was accredited to the reinforcement, being a rigid ceramic phase in a plastically deforming matrix, risking cavitation and whisker fracture at lower temperatures. In addition, safe regions of the A356 alloy and the A356 reinforced with 5 wt.\% $\mathrm{B}_{4} \mathrm{C}$ composite were studied in [174]. Maximum efficiency region was observed to decrease for the composite, which emphasizes the impact of the nature of reinforcement.

\subsection{3-D processing maps}

The effect of strain could not be well stated in Fig.8.3 and Fig.8.4 due to the discontinuity of the maps when showing results for each strain value separately. Thus, it is necessary to link the processing to some material's visual continuum model that can depict the continuity in deformation parameters. For this purpose, 3-D processing maps and flow instability maps were developed for the alloy and composite (Figs.8.5-8.8), having the advantage of identifying the workability conditions by reflecting the safe hot processing conditions of the whole deformation process directly. The three major influential parameters were targeted each at a time (i.e., $\varepsilon$ in 
Fig.8.5, $T$ in Fig.8.6, and $\dot{\varepsilon}$ in Fig.8.7). 3-D power dissipation maps were built every time, while introducing "slices" at chosen values of one of the previously mentioned parameters to better see its effect in a continuous manner.

Fig.8.5(a) and (b) represent the 3-D power dissipation maps of the $2024 \mathrm{Al}$ and the $2.0 \mathrm{wt} . \%$ $\mathrm{CNT} / 2024 \mathrm{Al}$ respectively, sliced at $\varepsilon=0.1, \varepsilon=0.3$, and $\varepsilon=0.5$. Effect of strain on the efficiency could be seen vertically through the variation of the grid levels, which proved that the efficiency of power dissipation varied reasonably with increasing strain. In fact $\eta$ increased with increasing strain at higher temperatures and lower strain rates or at lower temperatures and higher strain rates for both alloy and composite. The augmented $\eta$ values of the alloy compared to the composite were also well appreciated through the variation of the color flow.
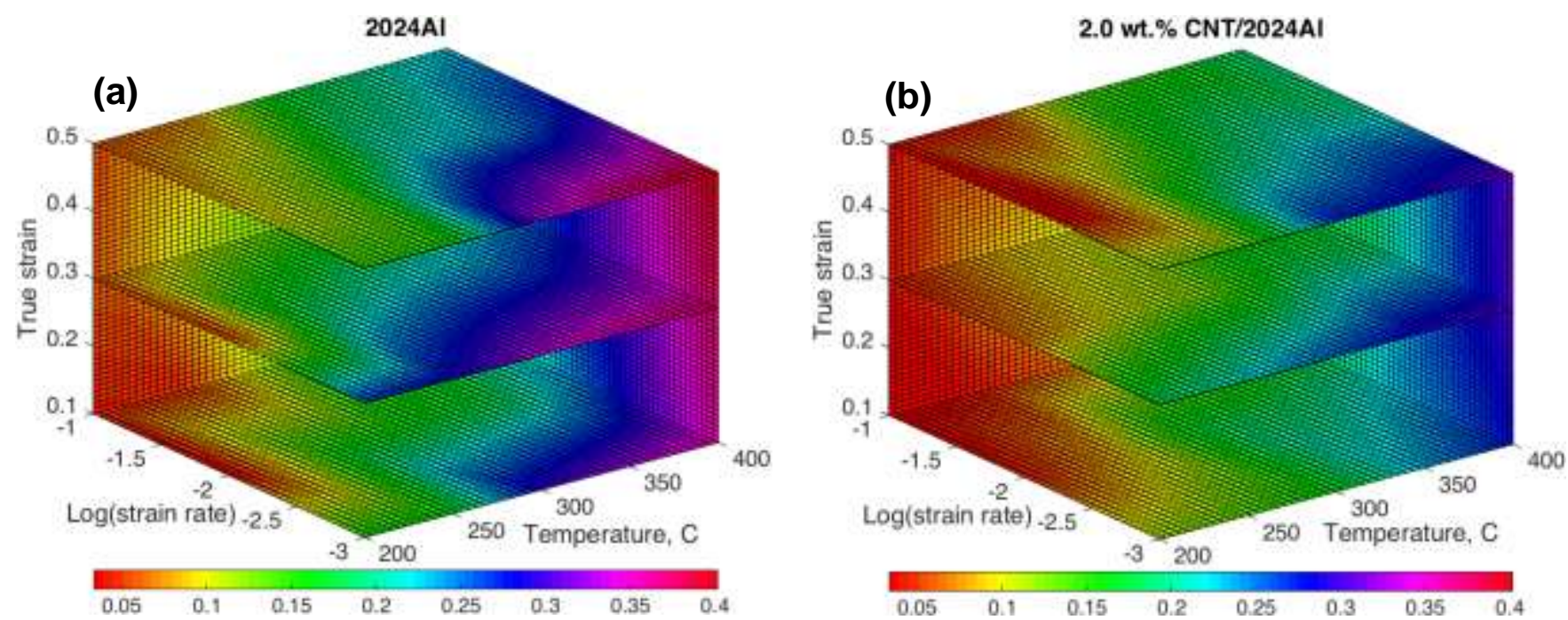

Fig.8.5 3-D power dissipation maps of (a) the 2024Al alloy, and (b) the $2.0 \mathrm{wt} . \% \mathrm{CNT} / 2024 \mathrm{Al}$ composite sliced at true strain values of $\varepsilon=0.1, \varepsilon=0.3$, and $\varepsilon=0.5$, respectively.

Fig.8.6(a) and (b) represent the second slicing option based on the temperature values. Three intermediate temperatures were chosen i.e., 200, 300, and $400^{\circ} \mathrm{C}$. The efficiency of power 
dissipation increased unceasingly with increasing temperature for both materials, where higher values were achieved by the alloy. On each of the chosen temperatures, $\eta$ values displayed an increasing flow towards lower strain rates.
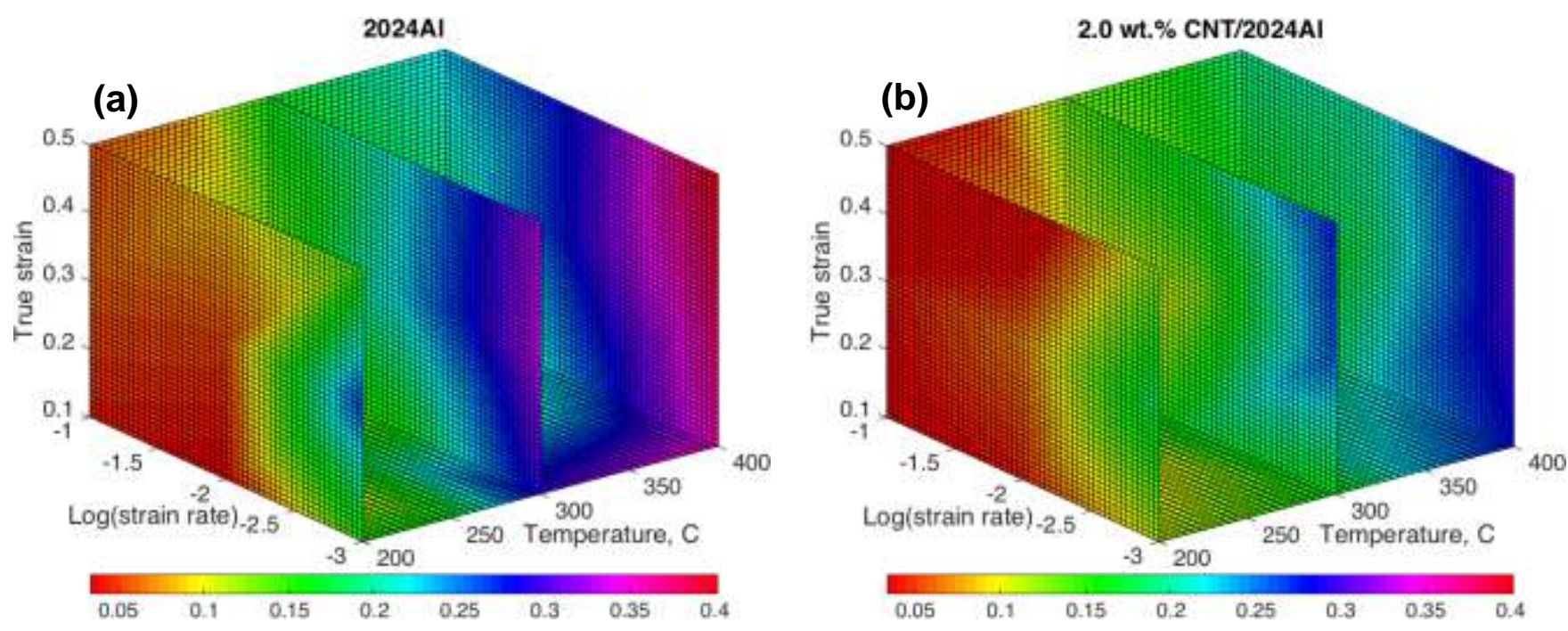

Fig.8.6 3-D power dissipation maps of (a) the 2024Al alloy, and (b) the 2.0 wt.\% CNT/2024Al composite sliced at temperature values of 200,300 , and $400^{\circ} \mathrm{C}$, respectively.

Fig.8.7(a) and (b) highlight the effect of strain rate on the efficiency values sliced at $0.001,0.01$, and $0.1 \mathrm{~s}^{-1}$. Efficiency clearly increased with decreasing strain rates for both materials. However, for the 2024Al in Fig.8.7(a), at the lowest strain rate of $0.001 \mathrm{~s}^{-1}$, two different regions that could not be previously seen in the 2-D maps, were identified. Values of $\eta \sim 20 \%$, were seen at lower temperatures $\left(200-275^{\circ} \mathrm{C}\right)$ at higher and lower strains, highlighting further the effect of strain on the processing maps. This allure was also depicted for the composite to some extent.

Based on the high efficiency regions observed in the 3-D maps, it could be inferred that the favorable conditions for hot processing persisted in the regions of lower strain rates and higher 
temperatures. It is known that domains with a higher efficiency of power dissipation commonly represent optimum processing conditions; however it is still primordial to observe the evolution of flow instabilities. Thus, 3-D flow instability maps were generated for the alloy and composite while considering only higher strain regions based on Fig.8.3 and 8.4. Slicing was made along $0.01,0.03$, and $0.1 \mathrm{~s}^{-1}$.
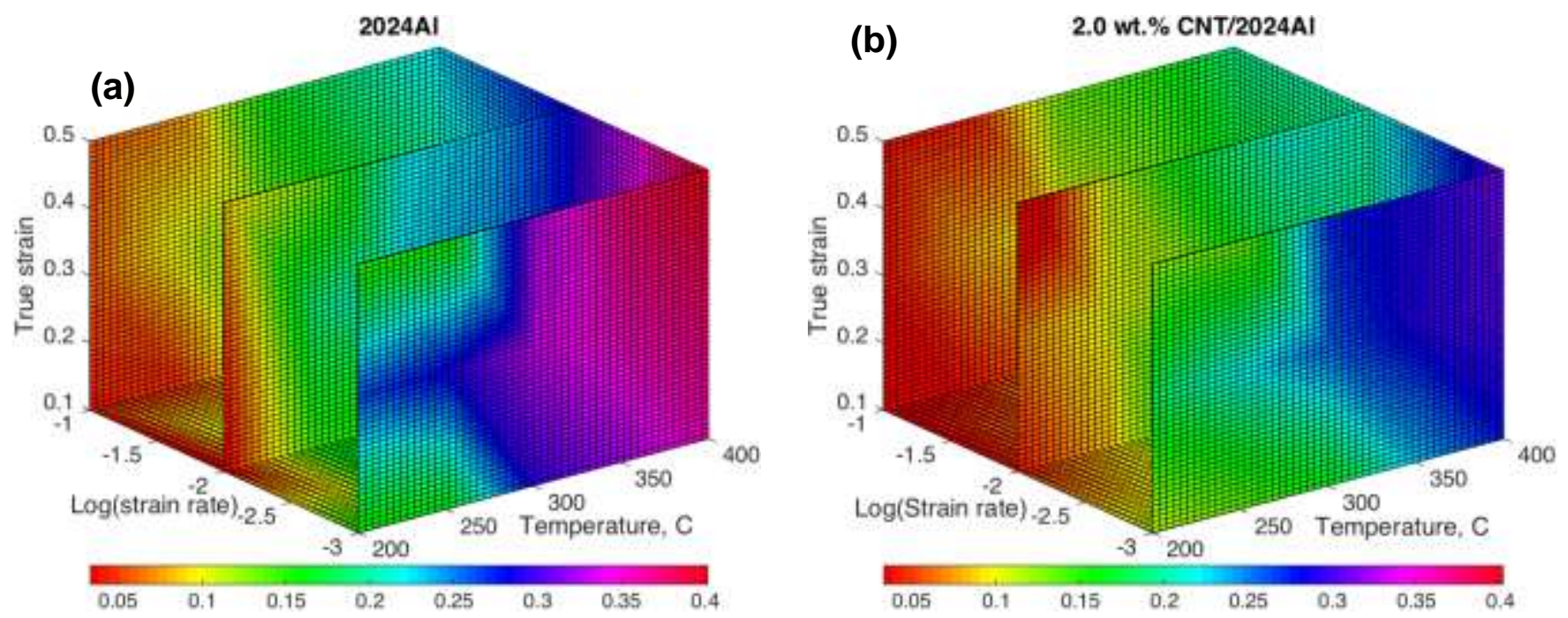

Fig.8.7 3-D power dissipation maps of (a) the 2024Al alloy, and (b) the $2.0 \mathrm{wt.} \% \mathrm{CNT} / 2024 \mathrm{Al}$ composite sliced at strain rate values of $0.001 \mathrm{~s}^{-1}(\log (\dot{\varepsilon})=-3), 0.01 \mathrm{~s}^{-1}(\log (\dot{\varepsilon})=-2)$, and $0.1 \mathrm{~s}^{-1}$ $(\log (\dot{\varepsilon})=-1)$, respectively.

Larger instable regions were observed for the composite at all the chosen strain rates. Most negative $\xi$ values were depicted around the temperature edges (i.e., $\sim 200^{\circ} \mathrm{C}$ ) in Fig.8.8(a). A bigger sensitivity to strain rate for the composite was seen, where the most negative $\xi$ values were observed at $0.1 \mathrm{~s}^{-1}$. The instability region almost vanished at $0.01 \mathrm{~s}^{-1}$ for the alloy, in contrast with the composite where although less negative values were seen compared to higher strain rates, the larger instable region persisted (Fig.8.8(b)). 

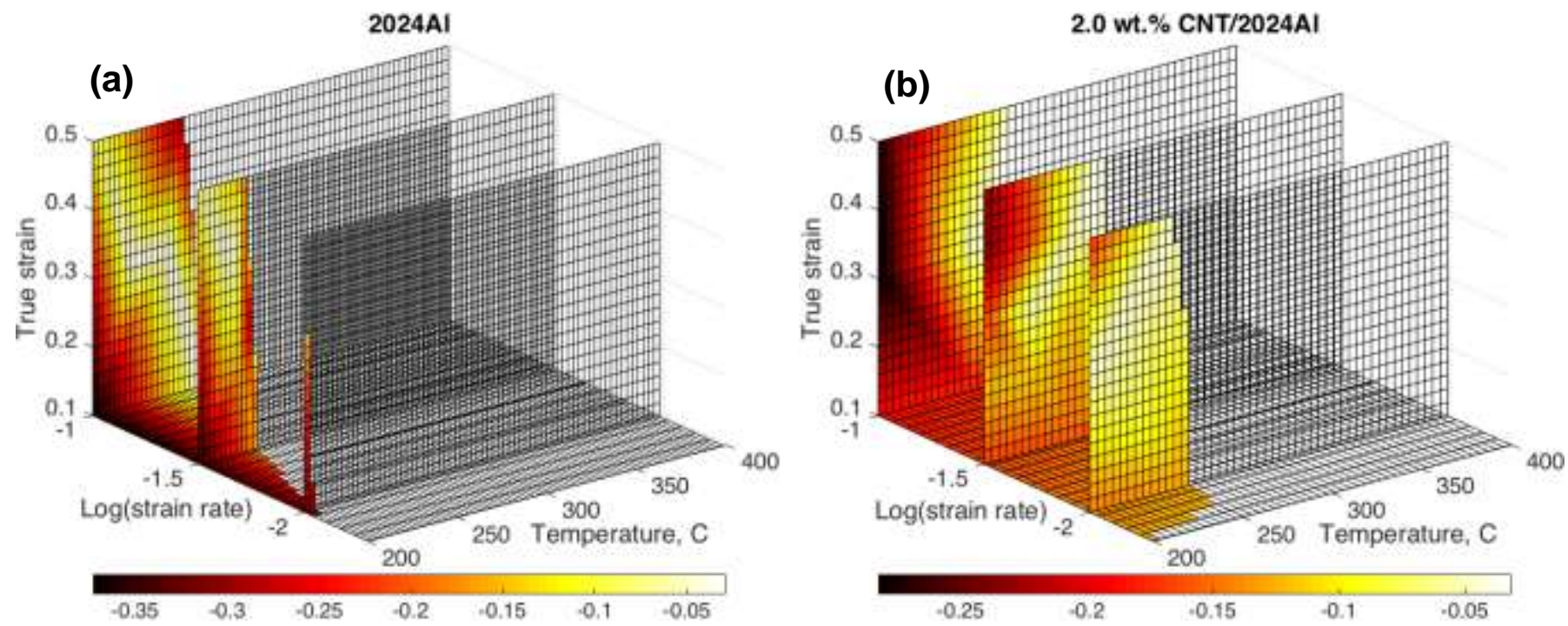

Fig.8.8 3-D flow instability maps of (a) the 2024Al alloy, and (b) the 2.0 wt. $\%$ CNT/2024Al composite sliced at strain rate values of $0.01 \mathrm{~s}^{-1}(\log (\dot{\varepsilon})=-2), 0.03 \mathrm{~s}^{-1}(\log (\dot{\varepsilon})=-1.5)$, and $0.1 \mathrm{~s}^{-1}$ $(\log (\dot{\varepsilon})=-1)$, respectively.

3-D Instability maps suggest that the hot workability of the alloy and composite became inferior as the strain rate increased; hence it became progressively difficult to deform the alloy and composite at higher strain rates. It is generally accepted that flow instability is associated with the localized shear initiated by high strain rates [166].

\subsection{Hot deformed microstructures}

\subsubsection{Deformed microstructures in the "unstable" domain}

Knowing that the increase in microstructural complexities could lead to narrowing down the hot working windows of the alloy and composite, it is crucial to understand the various 
microstructural aspects at different combinations of strain rates and temperatures. The current section is hence designated to the study of "instable" high temperature deformation features.

\subsubsection{Deformed 2024Al samples}

Fig.8.9(a) represents the SEM micrograph showing the overall microstructural features of the $2024 \mathrm{Al}$ alloy deformed at a strain rate of $0.1 \mathrm{~s}^{-1}$ and $200^{\circ} \mathrm{C}$.

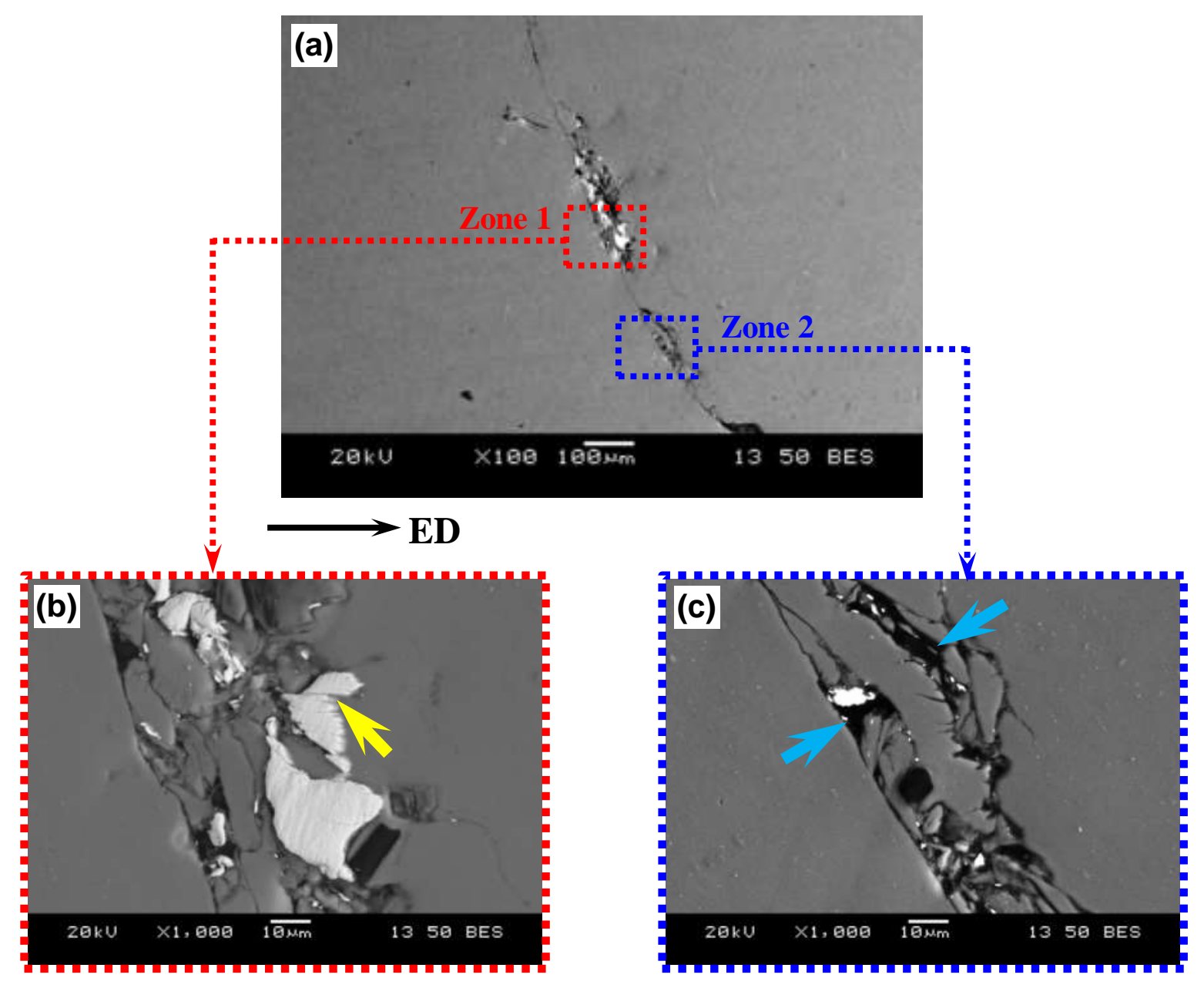

Fig.8.9 Typical SEM micrograph showing (a) the overall microstructural features of the 2024Al alloy deformed at a strain rate of $0.1 \mathrm{~s}^{-1}$ and $200^{\circ} \mathrm{C}$. Magnified micrographs of (b) zone1 and (c) zone 2 are also provided. 
A major crack was observed through the micrograph. Such regions are usually characterized by their inferior workability and may be induced by microstructural imperfections, such as micro/macro cracks, adiabatic shear bands, flow localization, wedge cracking, particle cracking and de-bonding [175]. Two different zones have been considered in Fig.8.9(a): Zone 1, magnified in Fig.8.9(b), and Zone 2 magnified in Fig.8.9(c). Micro-cracks were observed to propagate in Fig.8.9(b) and to affect the second phase particles, where the crack nucleated and propagated due to the presence of stress concentration caused by nonhomogeneous deformation at higher strain rates. As for Fig.8.9(c), the presence of voids (i.e., pointed out by arrows) was detected. These voids are generated between the "soft" matrix and the "hard" particles, originating macroscopic cracks from the surface to the interior linking the voids. Cracking could also be attributed to considerable stress concentration, caused by the sliding of neighboring grains under shear stress, where some micro cracks occur near the specimen surface and continue to propagate to macro cracks by linking each other.

Fig.8.10(a) represents a magnified view of the second-phase particle pointed out previously in Zone1 (Fig.8.9(b)). As mentioned earlier, coarser particles observed in the 2024Al were identified as $\mathrm{Al}_{2} \mathrm{Cu}$ particles. Several cracks were observed to occur in Fig.8.10(a) and tended to be parallel to the macroscopic compression axis (Along ED), indicating that fracturing was caused by local shear stresses. As schematized in Fig.8.10(b), two sorts of cracks were observed: major and minor cracks. Major cracks led to the lateral shift of the upper portion (part 1 in Fig.8.10(b)) of the particle along the compression axis, where a clear "particle cutting" was witnessed. The previous could be attributed to the dislocations moving along the primary slip planes. When larger compressive stresses are applied, part 1 and 2 in Fig.8.10(b) are expected to fully detach giving the illusion of the formation of two finer particles. 

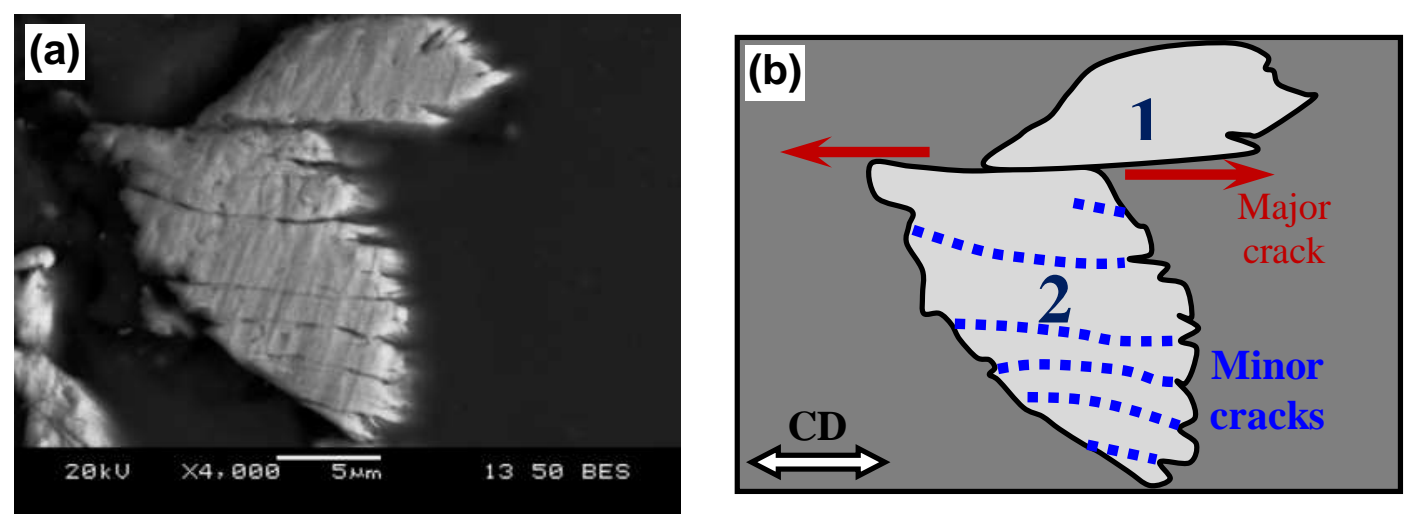

Fig.8.10 Second-phase particle cracking in the 2024Al base alloy compressed at $0.1 \mathrm{~s}^{-1}$ and $200^{\circ} \mathrm{C}$ : (a) SEM micrograph, and (b) schematic representation.

\subsubsection{Deformed 2.0wt.\% CNT/2024Al samples}

Fig.8.11(a) represent the SEM micrograph of the 2.0 wt.\% CNT/2024Al composite deformed at a strain rate of $0.1 \mathrm{~s}^{-1}$ and $200^{\circ} \mathrm{C}$. In addition to crack propagation through the observed surface, void formation in the composite was noted, as pointed out by the arrows in Fig.8.11(a). Similar observations were made in [176] for the $\mathrm{Al} 6063 / 0.75 \mathrm{Al}_{2} \mathrm{O}_{3} / 0.75 \mathrm{Y}_{2} \mathrm{O}_{3}$ composite where the microstructural damage consisted of cracks and debonding since the matrix undergoes plastic flow, while the particles do not deform. The previous was expected to lead to the reduction of the workability of the composite due to cavity formation, eventually contributing to ductile fractures. A specific region was selected in Fig.8.11(b). Two different types of cracks were observed: Minor cracks propagating along the ED (thin red arrows), and major cracks having an orientation of $\sim 45^{\circ}$ with respect to ED (thick green arrows). The different nature of these cracks could be linked to the loading along ED which induced a significant difference in the elastic moduli between the matrix and CNTs. This caused stress concentrations and inhomogeneous flow of the 
2024Al matrix, thus leading to the deep shearing micro-cracks (i.e., always inclined $45^{\circ}$ to the vector of the applied stress [177]) and the minor cracks parallel to ED.
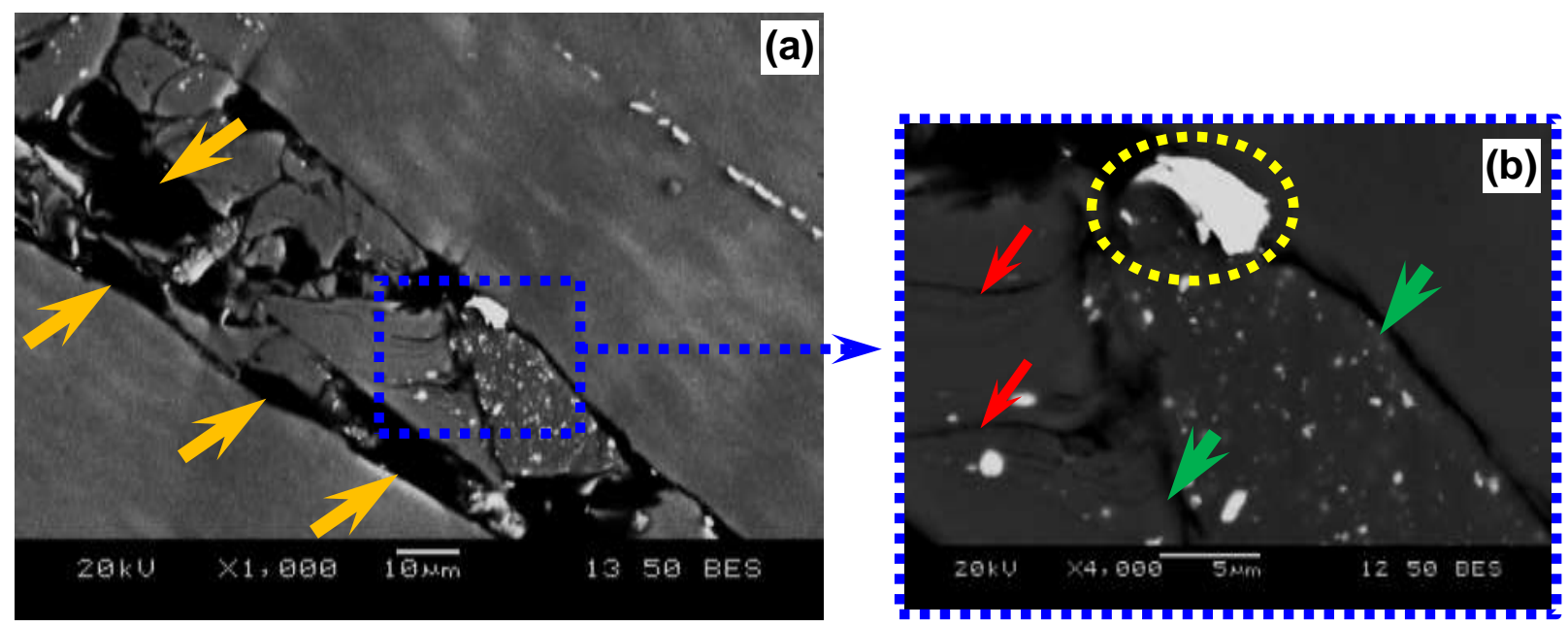

\section{ED}

Fig.8.11 Typical SEM micrograph showing (a) microstructural features of the $2.0 \mathrm{wt} . \%$ $\mathrm{CNT} / 2024 \mathrm{Al}$ composite deformed at a strain rate of $0.1 \mathrm{~s}^{-1}$ and $200^{\circ} \mathrm{C}$. Magnified view provided in (b).

The deformation stress generated near the matrix/SiC $\mathrm{C}_{\mathrm{P}}$ interface was also studied in [178] for the 20 vol. $\% \mathrm{SiC}_{\mathrm{P}} / 2024 \mathrm{Al}$. It was concluded that if deformation stress generated near the matrix/reinforcement interface does not have enough time to be accommodated at higher strain rates and exceeds the strength of the interface. Cavities would occur, grow, coalesce, and cause damage. Another observation could be made where the second phase particle, shown by dashed lines in Fig.8.11(b), displayed enhanced strength and resistance when compared to the particle previously studied in Fig.8.10 (i.e., only one crack plane was slightly depicted for the particle in Fig.8.11(b)). A similar observation was made in [169] where precipitates of the reinforced $\mathrm{Al}-\mathrm{Si}$ 
alloy were observed to be stronger than those in the base alloy and a less frequent cracking was reported.

Fig.8.12(a) represents the SEM micrograph of the 2.0wt.\% CNT/2024Al sample compressed at $400^{\circ} \mathrm{C}$ and $0.1 \mathrm{~s}^{-1}$. A clear rotation of second phase particles was noted with respect to the compression direction (ED shown in Fig.8.12). As schematized in Fig.8.12(b), and angle $\theta$ could be defined between the loading direction and the longitudinal axis of the particle.
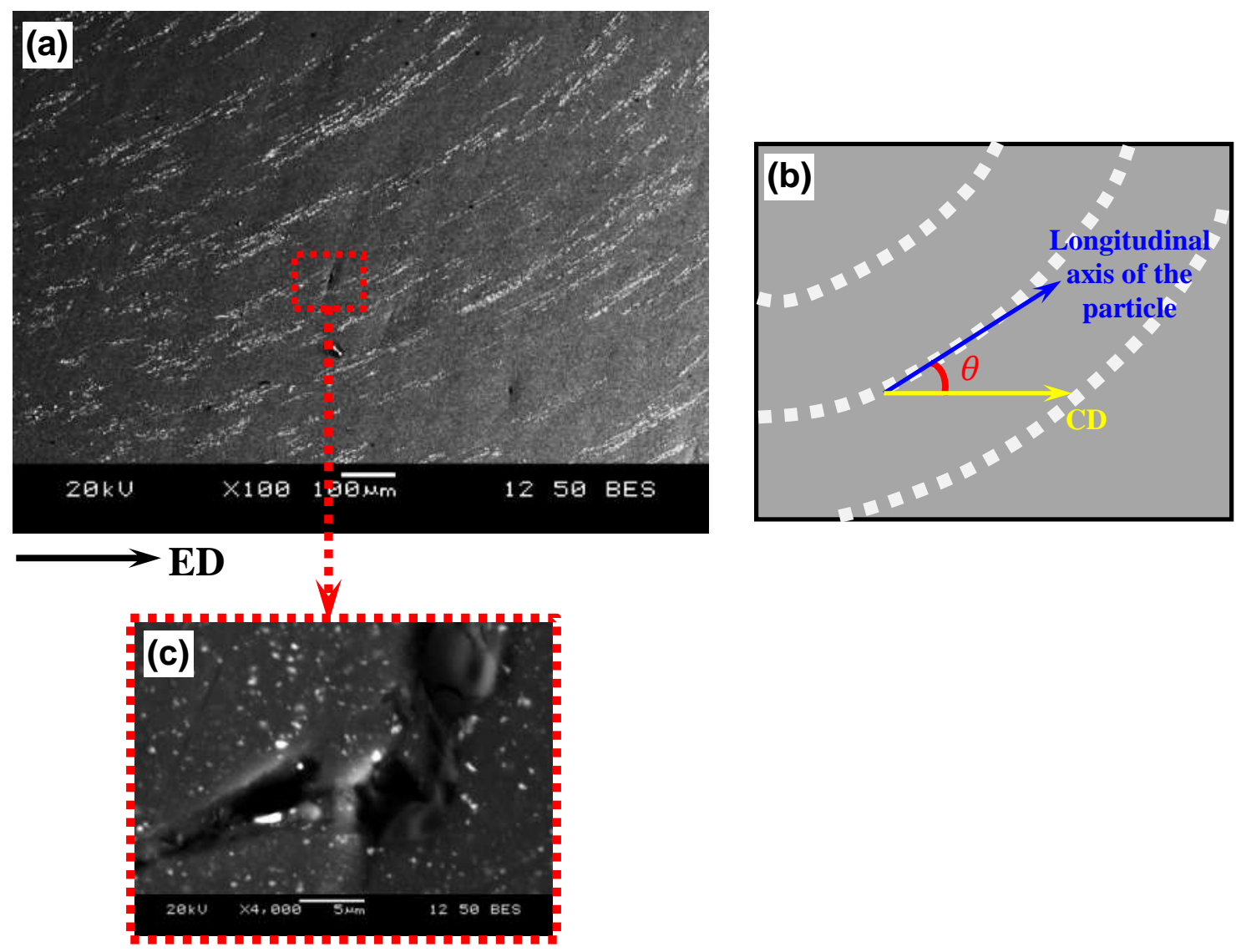

Fig.8.12 Typical SEM micrograph showing (a) second-phase particle rotation of the $2.0 \mathrm{wt} \%$ $\mathrm{CNT} / 2024 \mathrm{Al}$ composite deformed at a strain rate of $0.1 \mathrm{~s}^{-1}$ and $400^{\circ} \mathrm{C}$, (b) schematic representation, and (c) magnified view. 
A rotation of the Si particles was also observed for the Al-6-65Si-0.44Mg reinforced with $\mathrm{B}_{4} \mathrm{C}$ particulates where a schematic diagram of the particle alignment before and after compression described its rotation during material flow [174]. The orientation distribution of the eutectic $\mathrm{Si}$ particles after compression at a strain rate of $1 \mathrm{~s}^{-1}$ and $400^{\circ} \mathrm{C}$ was also provided in [169] where the fractions of precipitate of precipitate orientation based on the angle formed with the loading direction were quantified. Re-orientation of the particles with increasing deformation temperature was previously linked to eventual void nucleation, growth and coalescence [169]. To confirm that, the presence of some voids was detected in Fig.8.12(a) and was further magnified in Fig.8.12(c).

\subsubsection{Deformed microstructures in the "stable" domain}

Depending on the studied materials, microstructure mechanisms of the safe (i.e., stable) domains have previously been attributed to dynamic recrystallization (DRX), dynamic recovery (DRY) and superplasticity [171]. The following section is thus dedicated to studying the deformed microstructures in the stable domains. Using the efficiency of power dissipation and the dynamic stability criteria above, the safe hot working widow for the $2024 \mathrm{Al}$ and the 2.0 wt. $\%$ CNT/2024Al were found around higher temperatures and smaller strain rates. Hence, deformed microstructures and textures of the alloy and composite will be analyzed at 300,350 , and $400^{\circ} \mathrm{C}$, at a fixed strain rate of $0.001 \mathrm{~s}-1$. It is noted that for the 2024Al, EBSD analysis will be presented. An effort of obtaining deformed microstructures of the composite via EBSD was also attempted, however was not successful. In fact, it is already challenging to obtain accurate EBSD results of as-received MMCs, which made it even harder to analyze them after deformation, due to the 
poor fit of the solution to the pattern. Hence, XRD analysis will be relied on for the case of the composite.

\subsubsection{Deformed 2024Al samples}

Fig.8.13(a), (c), and (e) represent the post-deformed EBSD orientation maps for the $2024 \mathrm{Al}$ at $\dot{\varepsilon}=0.001 \mathrm{~s}^{-1}$, and temperature values of 300,350 , and $400^{\circ} \mathrm{C}$ respectively. Most of the grains in Fig.8.13(a) were equiaxed and an important grain size reduction was noticed in comparison with the as-received orientation map of the 2024Al (Fig.7.4(a)). Also more randomized orientations were observed in Fig.8.13(a) in comparison with Fig.8.13(c), where majority of the larger elongated grains exhibited a $<111>$ texture. Most of the elongated grains previously seen in Fg.7.4(a) vanished, expect a minority of grains which were pointed out by arrows in Fig.8.13(a). The corresponding cumulative histogram of grain dimeters displayed in Fig.8.13(b), showed a pick value at $\sim 3.4 \mu \mathrm{m}$, representing the size of majority of the grains. A clear grain refinement was noted compared to the as-received microstructure in Fig.7.4(a), previously found to be $\sim 5$ $\mu \mathrm{m}$ for most of the grains. Fig.8.13(c) represents the EBSD orientation map at a similar strain rate $(0.001 \mathrm{~s}-1)$ but at a higher temperature of $350^{\circ} \mathrm{C}$. Very few elongated grains persisted within a majority of equiaxed grains.

A more randomized grain orientation was observed, with a further refined grain size of $\sim 2.9 \mu \mathrm{m}$

for majority of the grains. As previously stated, a maximum efficiency of power dissipation of $\sim 39 \%$ was attained at $400^{\circ} \mathrm{C}$ and $0.001 \mathrm{~s}-1$ which corresponds to the condition of deformation of the map in Fig.8.13(e). All grains in Fig.8.13(e) were equiaxed. 
$300^{\circ} \mathrm{C}$
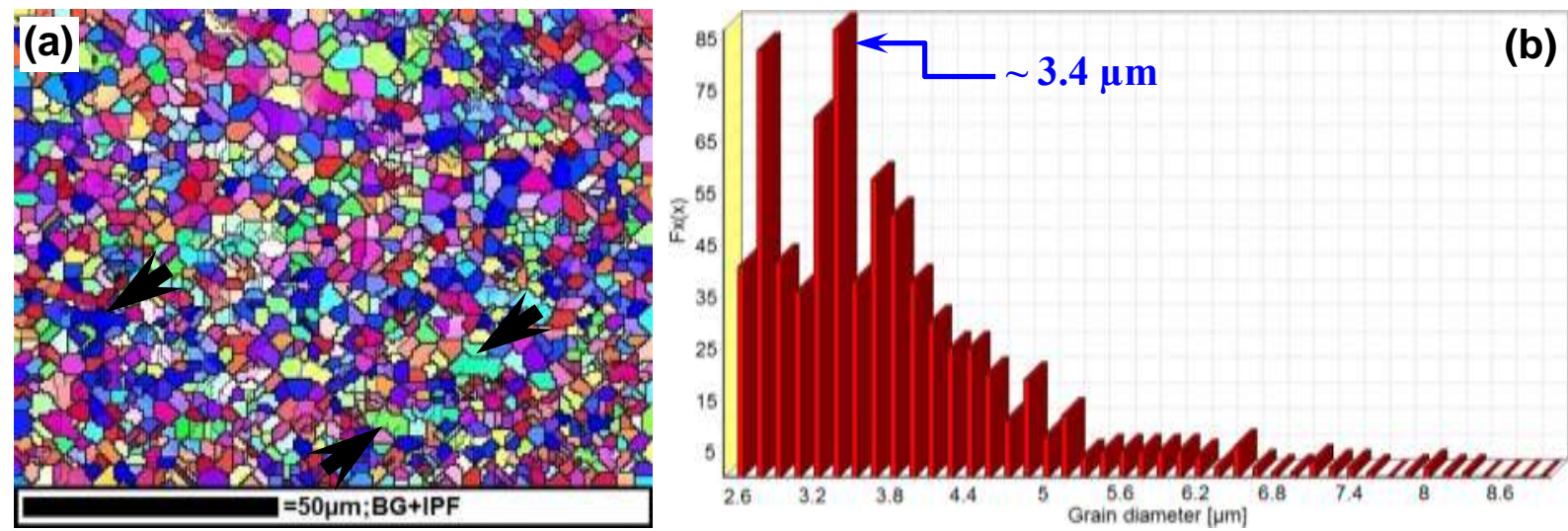

$350^{\circ} \mathrm{C}$
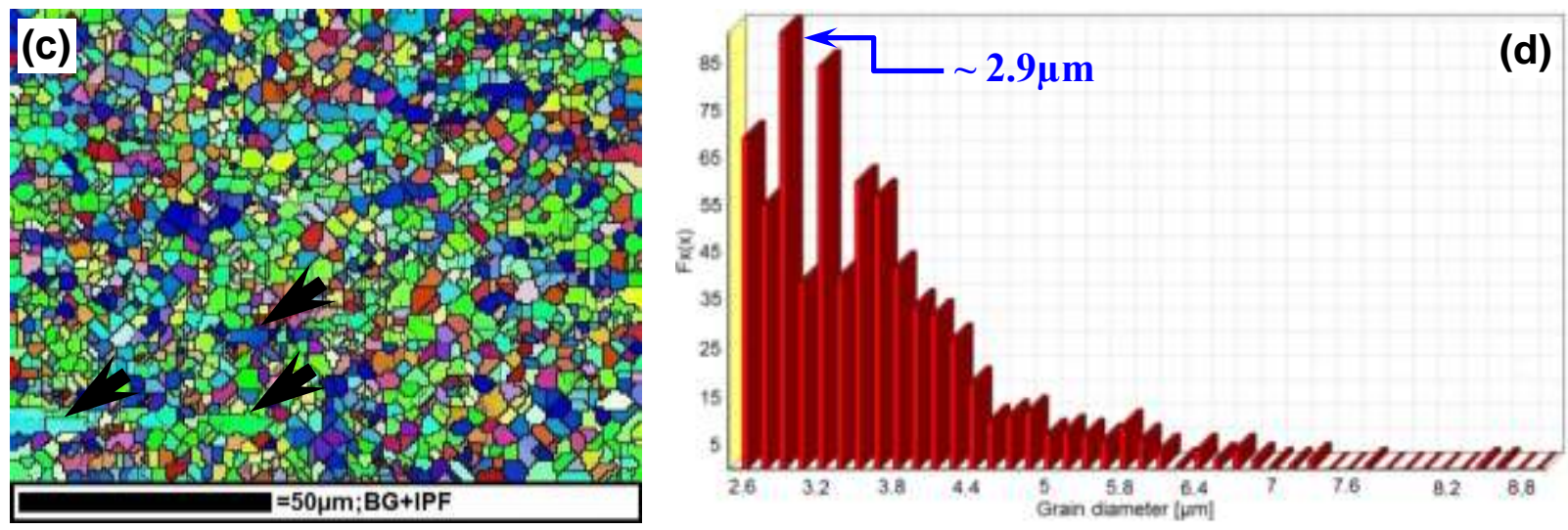

$400^{\circ} \mathrm{C}$
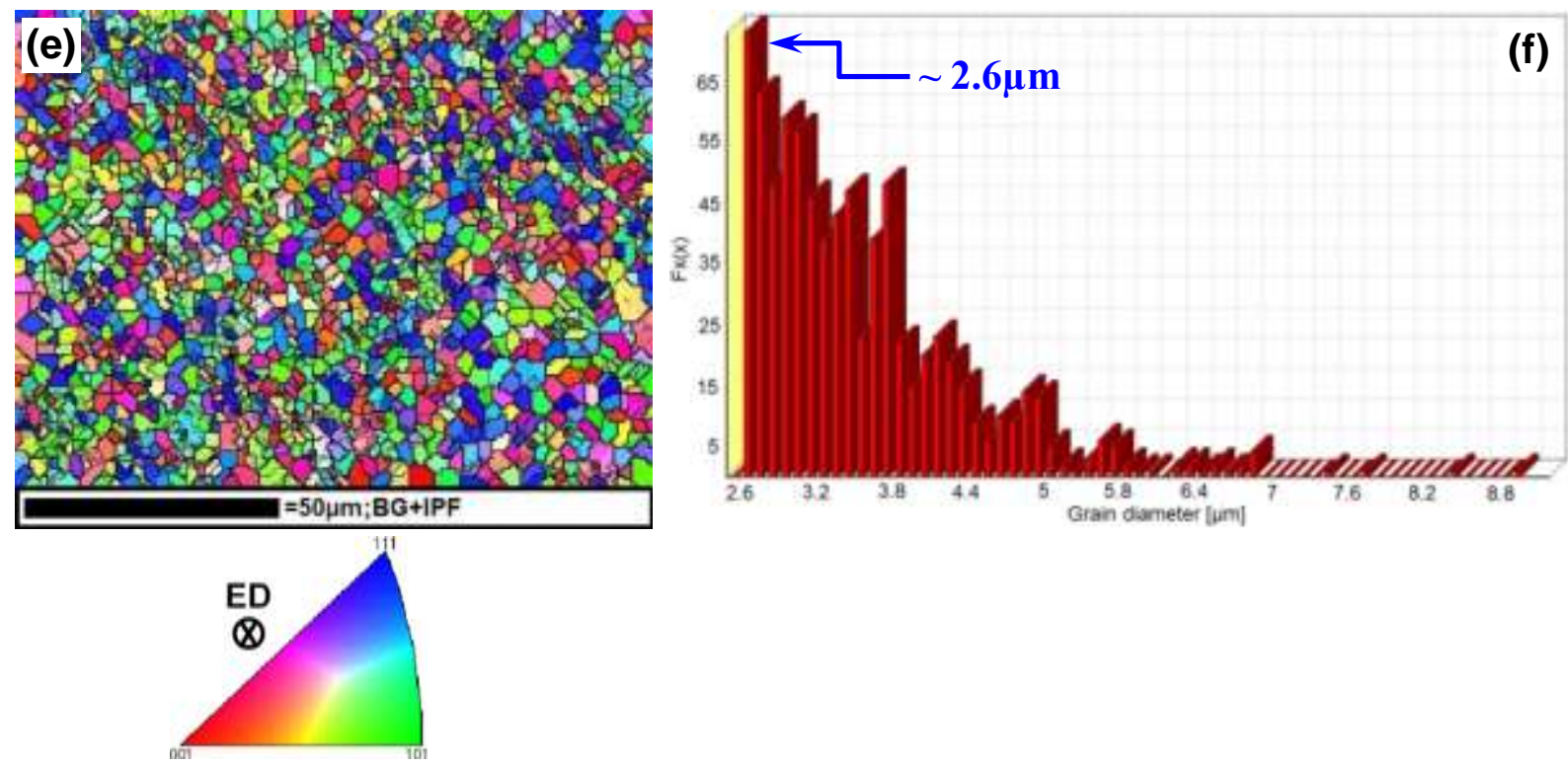

Fig.8.13 EBSD orientation maps along with grain diameter distribution histograms of the 2024Al alloy deformed at a strain rate of $0.001 \mathrm{~s}^{-1}$ and $(\mathrm{a}, \mathrm{b}) 300^{\circ} \mathrm{C}$, (c, d) $350^{\circ} \mathrm{C}$, and (e, f) $400^{\circ} \mathrm{C}$. 
A perfectly homogeneous microstructure was observed and no remaining elongated grains were seen. Fig.8.13(f) displayed a further refinement of the grain size (a peak of $\sim 2.6 \mu \mathrm{m}$ ). The homogeneous grain size distribution displayed in the optimal conditions of Fig. 8.13(e), indicated the occurrence of adequate DRX [166]. This confirms that higher temperatures and lower strain rates are preferred for the hot working of the 2024Al. The motivation behind this could be credited to the fact that at higher temperatures and lower strain rates, the DRX occurred more extensively with the progress of deformation, which helped reduce the flow localization and led to a better workability of the material. As mentioned above, DRX was reported to happen at lower strain rates above $250^{\circ} \mathrm{C}$ in [167]. It was also mentioned that up to a deformation of $425^{\circ} \mathrm{C}$, the 2024Al exhibited fine equiaxed grains near the alignments of $(\mathrm{Cu}, \mathrm{Al})$ particles and many transverse subgrains which are indicative of the persistence of DRX, were noted, however, starting from a temperature of $482^{\circ} \mathrm{C}$, abnormal grain growth started to be recorded [167], i.e., much higher than our temperature limit of $400^{\circ} \mathrm{C}$ in the current study.

Another EBSD feature was exploited in Fig.8.14 where deformed microstructures were fractioned into recrystallized, substructured and deformed portions. The partition is realized through AztecHKL software such as recrystallized grains could be identified in a set of plastically deformed grains by the grain orientation spread (GOS) method, where the internal average misorientation angle within each grain is measured. EBSD recrystallized fraction components of the specimens deformed in the safe region were thus provided in Fig.8.14(a), (c) and (e) for 300,350 , and $400^{\circ} \mathrm{C}$ respectively. Corresponding quantification histogram of each of the maps was also provided where the recrystallized fraction was observed to increase from $\sim 36 \%$ (Fig.8.14(b)) to $\sim 38 \%$ (Fig.8.14(d)), to $\sim 46$ (Fig.8.14(f)). It is noted that the increase observed 
between the maps for 300 to $350^{\circ} \mathrm{C}$ was less important (only $2 \%$ increase), however an important gain in the recrystallized fraction was observed in Fig.8.14(f), where majority of the grains in Fig.8.14(e) were recrystallized. This was expected since a maximum efficiency of power dissipation of $\sim 39 \%$, was attained at $400^{\circ} \mathrm{C}$ and $0.001 \mathrm{~s}^{-1}$ introducing a homogenized microstructure. The various EBSD features exploited throughout this section proved that continuous DRX was essentially observed where new grains nucleated evenly at high temperatures and low strain rate throughout the material and could hardly grow, since as stated above, the grain growth for the $2024 \mathrm{Al}$ alloy could only be seen at temperatures above the $482^{\circ} \mathrm{C}$ threshold [167]. For the metals with high stacking fault energy such as $\mathrm{Al}$, the main mechanism for DRX is continuous dynamic recrystallization (CDRX) at higher deformation temperatures [179], and the new grains could be formed gradually by transforming the low angle grain boundaries to high angle grain boundaries. Therefore, the peak efficiency of power dissipation may be mainly attributed to grain refinement by CDRX.

\subsubsection{Deformed 2.0 wt.\% CNT/2024Al samples}

Fig.8.15(a) represents the initial $\{110\}$ pole figure of the $2.0 \mathrm{wt} . \% \mathrm{CNT} / 2024 \mathrm{Al}$ composite where majority of the poles were aligned parallel to RD and a maximum intensity of $\sim 4.38$ MRD was observed. After a compression at $300^{\circ} \mathrm{C}$ and $0.001 \mathrm{~s}^{-1}$, a split of intensity was observed and poles $\mathrm{P}_{2}, \mathrm{P}_{3}, \mathrm{P}_{4}$, and $\mathrm{P}_{5}$ appeared in Fig.8.15(b), while being accompanied by an intensity decrease (3.01 MRD) due to the split of intensities between all the newly appeared poles. A texture randomization was also noted at $300^{\circ} \mathrm{C}$. 

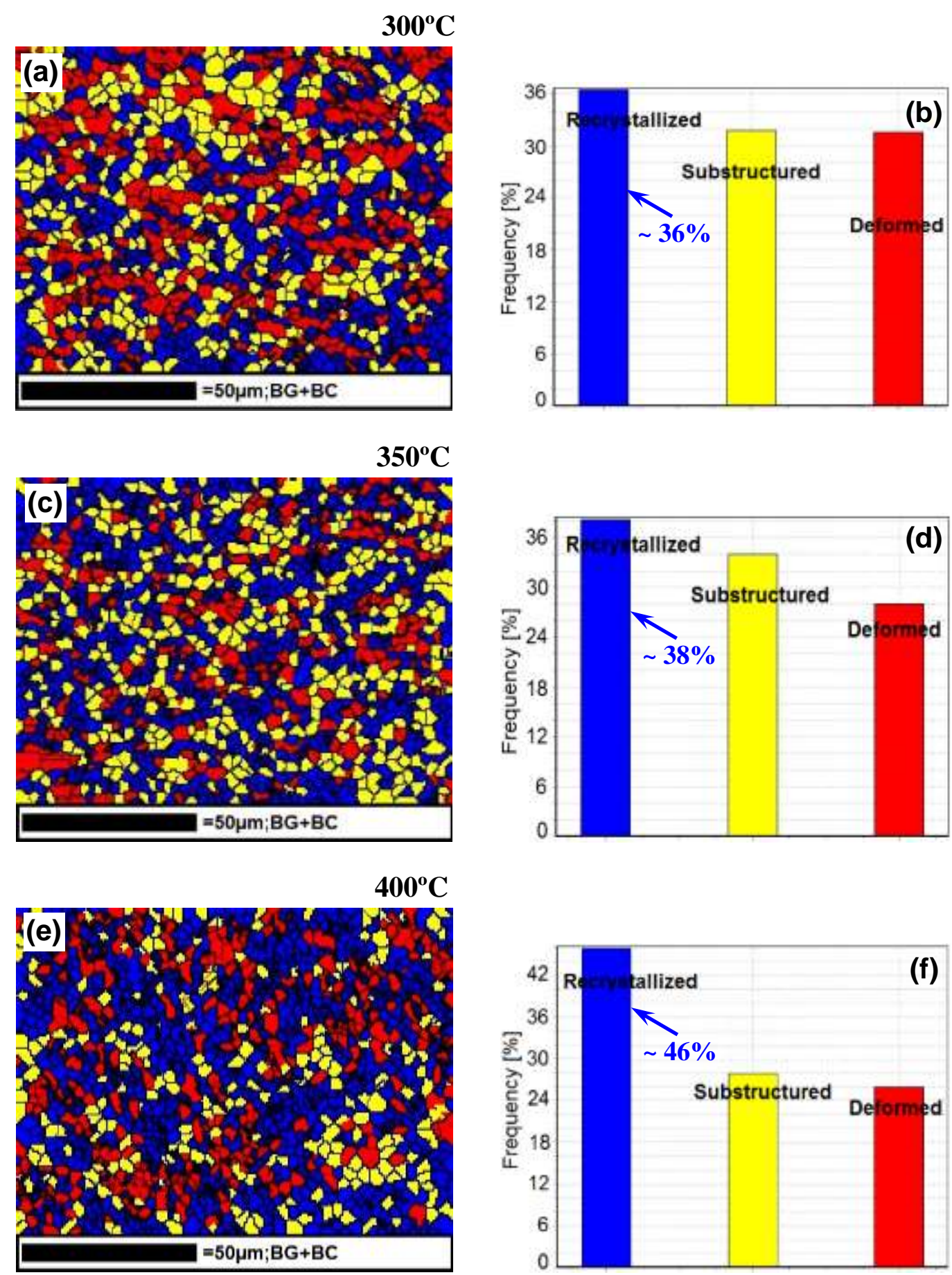

Fig.8.14 EBSD recrystallized fraction component maps along with their quantification histograms of the $2024 \mathrm{Al}$ alloy deformed at a strain rate of $0.001 \mathrm{~s}^{-1}$ and $(\mathrm{a}, \mathrm{b}) 300^{\circ} \mathrm{C},(\mathrm{c}, \mathrm{d})$ $350^{\circ} \mathrm{C}$, and (e, f) $400^{\circ} \mathrm{C}$. 
The intensity decrease continued in Fig.8.15(c) for the composite deformed at $350^{\circ} \mathrm{C}$ and the same strain rate, however the shifting towards $\mathrm{P}_{2}$ and $\mathrm{P}_{3}$ was more obvious.

(a) Initial

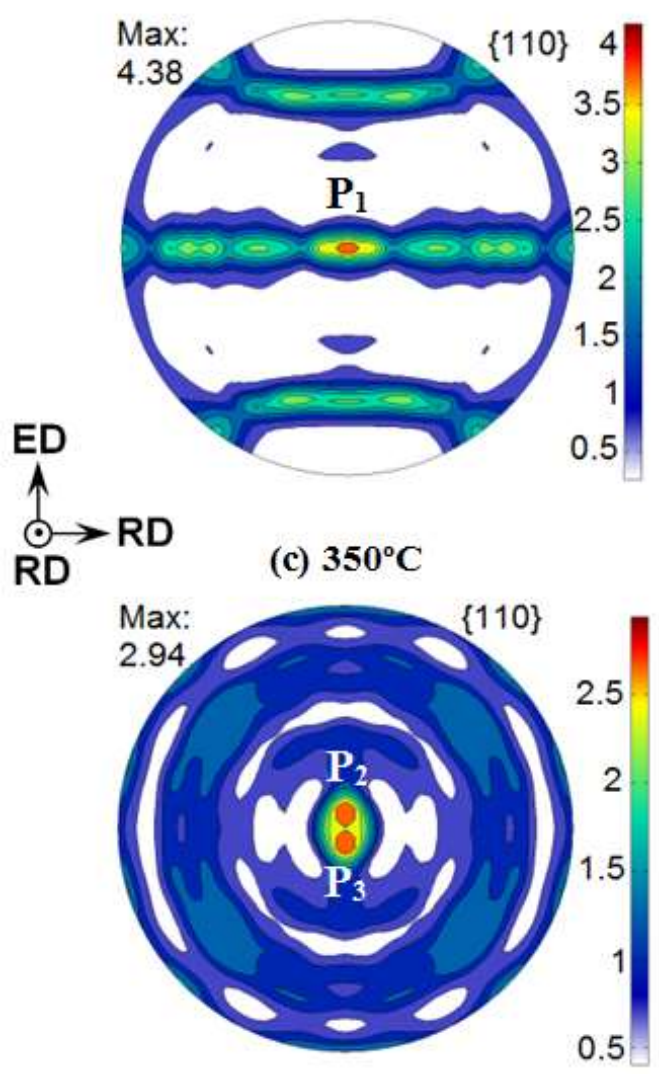

(b) $300^{\circ} \mathrm{C}$

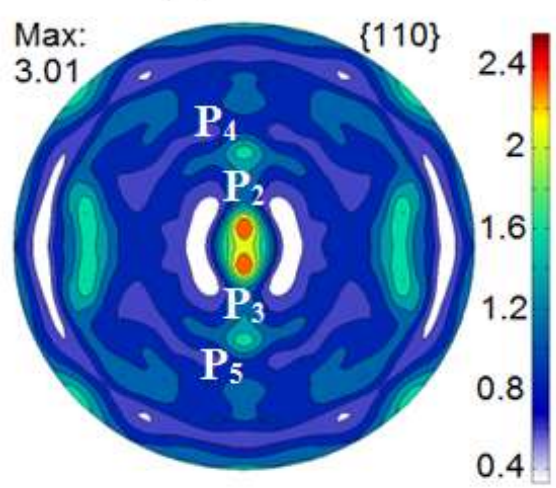

(d) $400^{\circ} \mathrm{C}$

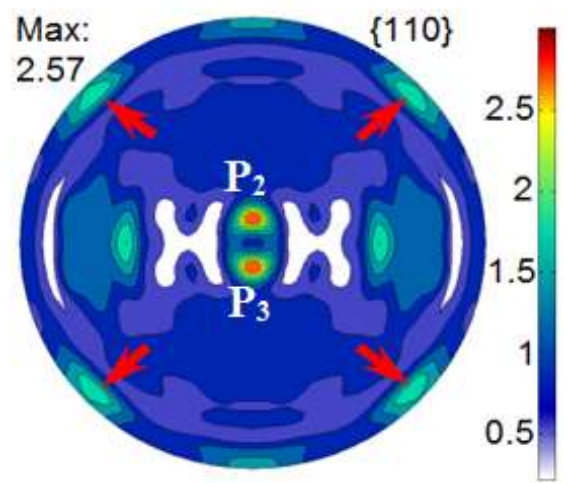

Fig.8.15 $\{110\}$ pole figures of the $2.0 \mathrm{wt} . \% \mathrm{CNT} / 2024 \mathrm{Al}$ composite at (a) the undeformed state, and compressed at a strain rate of $0.001 \mathrm{~s}^{-1}$ and (b) $300^{\circ} \mathrm{C}$, (c) $350^{\circ} \mathrm{C}$, and (d) $400^{\circ} \mathrm{C}$.

As previously stated, a maximum efficiency of power dissipation of $\sim 30 \%$, was attained at $400^{\circ} \mathrm{C}$ and $0.001 \mathrm{~s}^{-1}$ for the composite which corresponds to the condition of deformation of Fig.8.15(d). An accented intensity spread between $\mathrm{P}_{2}$ and $\mathrm{P}_{3}$ was obvious. Although $\mathrm{P}_{2}$ and $\mathrm{P}_{3}$ were observed to sharpen in Fig.8.15(d), the intensity continued to decrease since few cubic orientations started to develop as well (Pointed by arrows in Fig.8.15(d)), leading to a further slip of intensities. DRX texture components could be identified for the composite through the two 
poles $\mathrm{P}_{2}$ and $\mathrm{P}_{3}$. DRX was also observed for high temperature deformed microstructures of composite fabricated with finer $\mathrm{SiC}_{\mathrm{P}}$ and smaller 2024Al powders [178]. It was reported in [180] that the 2024Al with $8 \mu \mathrm{m}$ SiCp exhibited small equiaxed grains with high dislocation density at temperatures of 320-400, indicating that DRX occurred during hot deformation. Textures of deformed samples at $350{ }^{\circ} \mathrm{C}$ and $450^{\circ} \mathrm{C}$ of the $\mathrm{AZ61} / \mathrm{Sr}$ were also considered in [181]. In case of increasing temperature, the texture changed to random with a lower intensity, which is conforming to the observations made in Fig.8.15.

\subsection{Summary}

The modeling of material behavior which explicitly describes the dynamic processes occurring during hot deformation has been presented for the 2024Al base alloy and the 2.0 wt.\% $\mathrm{CNT} / 2024 \mathrm{Al}$ composite by means of high temperature compression in the range $200-400^{\circ} \mathrm{C}$, and 0.001-0.1 s $\mathrm{s}^{-1}$. The following conclusions could be drawn:

1. The presence of CNTs in the 2024Al alloy increased the activation energy of its plastic deformation from $275 \mathrm{~kJ} / \mathrm{mol}$ to $322 \mathrm{~kJ} / \mathrm{mol}$, suggesting the beneficial role of the reinforcement in improving its high temperature performance.

2. 3-D processing maps were able to deal with the sensitivity of the workability to strain at elevated temperatures, which helped to clearly describe the distributions of the efficiency of power dissipation and flow instability domains in terms of the process parameters without interruptions.

3. According to the conventional 2-D and 3-D processing maps, single optimum domains of hot deformation for the alloy and composite were detected in the high temperature, low strain 
rate range, with a shrinking of the processing window limits for the composite, due to its more complicated microstructural features at high temperature deformation.

4. The processing map of the $2024 \mathrm{Al}$ alloy and the $2.0 \mathrm{wt} . \% \mathrm{CNT} / 2024 \mathrm{Al}$ revealed a maximum efficiency of power dissipation of $\sim 39 \%$ and $\sim 30 \%$, respectively, which were both obtained $400^{\circ} \mathrm{C}$ and $0.001 \mathrm{~s}^{-1}$.

5. Instability regions occurred at higher strain rates and lower temperatures in both alloy and composite. However, it almost vanished at $0.01 \mathrm{~s}^{-1}$ for the alloy, in contrast to the composite where larger instable regions persisted.

6. The study of the stable regions of the $2024 \mathrm{Al}$ at the lowest strain rate of $0.001 \mathrm{~s}^{-1}$ and the temperature values of 300,350 , and $400^{\circ} \mathrm{C}$ via $\mathrm{EBSD}$ revealed an increase of the recrystallized fraction from $\sim 36 \%$, to $\sim 38 \%$, to $\sim 46 \%$, respectively, where a homogenized microstructure having equiaxed grains was observed at the highest temperature.

7. Although second phase particles in both alloys had a similar morphology, there was a difference in their performance during hot compression. In the composite, they were more resistant to the induced micro-cracks. 


\section{CHAPTER 9}

\section{CONCLUSIONS AND FUTURE WORK}

\subsection{Conclusions}

The proposed dissertation helped understand the factors affecting the deformation behavior and performance of two key lightweight alloys in automotive applications, namely $\mathrm{Al}$ and $\mathrm{Mg}$ alloys. Investigations started by examining the deformation behavior of RE-free wrought $\mathrm{Mg}$ alloys, through RT compression tests of an extruded AZ31 Mg alloy. Then, a recently-developed ZEK100 Mg alloy with a low RE content (i.e., 0.2 wt.\% Nd) was subjected to strain-controlled push-pull type fatigue tests at a constant strain rate and RT, while being compared with RE-free and high RE-containing alloys. Both cyclic and anelastic behavior were evaluated. Effect of CNT additions was also appreciated through RT and HT compression tests on a 2024Al base alloy and a $2.0 \mathrm{wt} . \% \mathrm{CNT} / 2024 \mathrm{Al}$ composite. The following major conclusions could be drawn:

1. Plastic deformation of the extruded AZ31 Mg alloy deformed under compression along the extrusion direction was characterized by profuse extension twinning.

2. Twinning propagation in wrought $\mathrm{Mg}$ alloys was accommodated by the growth of existing twin lamellas at higher compressive strains. A fast saturation of twin nucleation was attained for the case of single twin variants. However, for double twin variants, intersecting twins led to a delayed space confinement by the finer twin lamellas.

3. The microstructure of a low RE-containing ZEK100 Mg alloy in the annealing O condition was characterized by equiaxed grains due to the occurrence of DRX. The alloy contained 
some $\mathrm{Mg}_{12} \mathrm{Nd}$ and $\mathrm{MgZn}$ particles. The addition of $0.2 \mathrm{wt} . \% \mathrm{Nd}$ along with $1.3 \mathrm{wt} . \% \mathrm{Zn}$ and 0.25 wt.\% $\mathrm{Zr}$ led to the weakening of basal texture in comparison with the extruded RE-free AZ31 alloy.

4. Fatigue life of ZEK100 determined via strain-controlled fatigue tests was longer than that of the extruded RE-free Mg alloys, as a result of a fairly good combination of strength and ductility. A slight cyclic softening was observed at high strain amplitudes, whereas cyclic stabilization occurred at lower strain amplitudes.

5. A major improvement in the asymmetry of the ZEK100 hysteresis loops was observed in comparison with the extruded RE-free Mg alloys, indicating the effectiveness of the addition of $\mathrm{Nd}$ which played a significant role in overpowering the incidence of excessive twinning via texture weakening and grain refinement.

6. Global sensitivity analysis indicated that strain ratio was an influential parameter for the anelastic behavior. The anelastic strain amplitude, along with the three newly defined parameters (eccentricity, angle deviation, and relative slope change) all increased with decreasing strain ratio, reflecting more asymmetric and skewed hysteresis loops.

7. Singly dispersed CNTs in the $2024 \mathrm{Al}$ matrix were revealed via TEM. $\mathrm{Al}_{4} \mathrm{C}_{3}$ phase was also present, being either directly attached to CNTs or in the matrix in their vicinity, suggesting the occurrence of reactions between the $\mathrm{Al}$ matrix and CNTs during the composite synthesis.

8. Deformation resistance of the composite at both RT and HT was effectively enhanced owing to the addition of CNTs. This was mainly associated to the Hall-Petch strengthening, and composite strengthening including load transfer, thermal mismatch and Orowan looping. 
9. The presence of CNTs in the $2024 \mathrm{Al}$ alloy increased the activation energy of its plastic deformation from $275 \mathrm{~kJ} / \mathrm{mol}$ to $322 \mathrm{~kJ} / \mathrm{mol}$, suggesting the beneficial role of the reinforcement in improving its high temperature performance. Also, according to the conventional 2-D and 3-D processing maps, single optimum domains of hot deformation for the alloy and composite were detected in the high temperature, low strain rate range, with a shrinking of the processing window limits for the composite, due to its more complicated microstructural features at high temperature deformation.

\subsection{Main contributions}

The proposed research was fundamental in dealing with some of the most challenging barriers facing the development and applications of lightweight alloys. Major contributions consisted of addressing a few critical issues and limitations faced by $\mathrm{Mg}$ and $\mathrm{Al}$ alloys through the consideration of RE addition and CNT reinforcement. A fundamental understanding of deformation and fatigue of lightweight alloys was developed in order to achieve long-term performance, provide a basis for further development of lightweight alloys for automotive, aerospace, and electronic applications. The following major contributions could be listed:

- The occurrence of deformation twinning in wrought Mg alloys has been well recognized, however its effect on the mechanical behavior under stepwise uniaxial loading has not yet been well understood. This research conducted fundamental studies on the mechanical behavior in wrought Mg alloys, which are essential for the implementation of lightweight structural applications in the manufacturing sectors, since twinning plays a significant role in their deformation and failure. 
- Various double twinning structures that were only acknowledged theoretically or through atomistic simulations (e.g., molecular dynamics) were detailed experimentally through in-situ EBSD investigations of similar regions throughout stepwise compression tests.

- New twin-twin interaction scenarios (i.e., ladder-like and branching-like structures) were identified depending on the strain amounts and paths of incoming twins and their impingement on the pre-existing twins.

- Two of the manuscripts published during this research about the fatigue of ZEK100 Mg alloy were the first studies in the literature to cover the LCF of such a low RE-containing alloy. In fact, the major impact was to provide an option that promotes the use of low cost and low RE-Mg alloys due to their improved mechanical properties.

- Since the higher the weight percent of RE elements is, the pricier the alloy would be, cost was taken into account by improving the properties of an $\mathrm{Al}$-free and low $(0.2 \mathrm{wt} . \% \mathrm{Nd})$ RE-containing ZEK100 wrought Mg alloy and by detailing different aspects of its fatigue performance in comparison with $\mathrm{RE}$-free $\mathrm{Mg}$ alloys and rich RE-containing (highly expensive) Mg alloys, thus promoting ZEK100 as an affordable option for auto industry.

- Investigations on the material behavior through hot deformation of $\mathrm{Al}$ base alloy and CNT-reinforced AMCs put emphasis on the impact of CNTs on the strength and ductility of the Al base alloys. The performed tests underlined the impact of CNTs on hot workability, activation energy, and optimum processing window, which constitute a benchmark for future work on these materials. 


\subsection{Recommendations for future work}

The following future investigations could further deepen the mechanistic understanding of the unusual plastic deformation behavior in wrought $\mathrm{Mg}$ and $\mathrm{Al}$ alloys during monotonic and cyclic loading:

1. Digital image correlation would be interesting in tracking the local strain distribution during stepwise compression tests of RE-free Mg alloys.

2. Only stepwise compressions along ED were presented while studying twinning deformation in the present work. It would be also interesting to use the orthorhombicshaped sample and vary the loading direction to examine the behavior of twinning. Detwinning (i.e., twin shrinkage) is expected to occur and would be interesting to monitor through in-situ EBSD.

3. Polycrystal plasticity modeling combined with TEM observations would be helpful in understanding the hardening behavior in Mg alloys.

4. Twin-twin interaction using 3D-XRD and tomography could provide a better understanding of the twinning mechanisms in $\mathrm{Mg}$.

5. Bi-axial or multi-axial fatigue tests for the RE-Mg alloys would be interesting to study the anisotropic behavior of these alloys, since no such results have been reported in the literature for these alloys yet.

6. It would be interesting to predict the cyclic deformation behavior of RE-Mg alloys using finite element analysis, along with a quantitative correlation between the strain hardening, twinning, and texture of RE-Mg alloys. 
7. Microstructural characterization of the RE-Mg alloys with heat treatment, e.g., twin boundary structures, twin-dislocation and twin-precipitate interactions at an atomic level, needs to be done using HRTEM, high-temperature XRD, and if possible, using neutron diffraction.

8. Higher temperatures (up to $500^{\circ} \mathrm{C}$ ) while studying the hot deformation of $\mathrm{Al}$ base alloys and composites and to observe the microstructural features since abnormal grain growth is expected to occur at temperatures higher than $482^{\circ} \mathrm{C}$ for such material. 


\section{APPENDICES}

\section{HOT DEFORMATION FLOW CURVES AT ALL STRAIN RATES AND TEMPERATURES FOR THE ALLOY AND THE COMPOSITE}
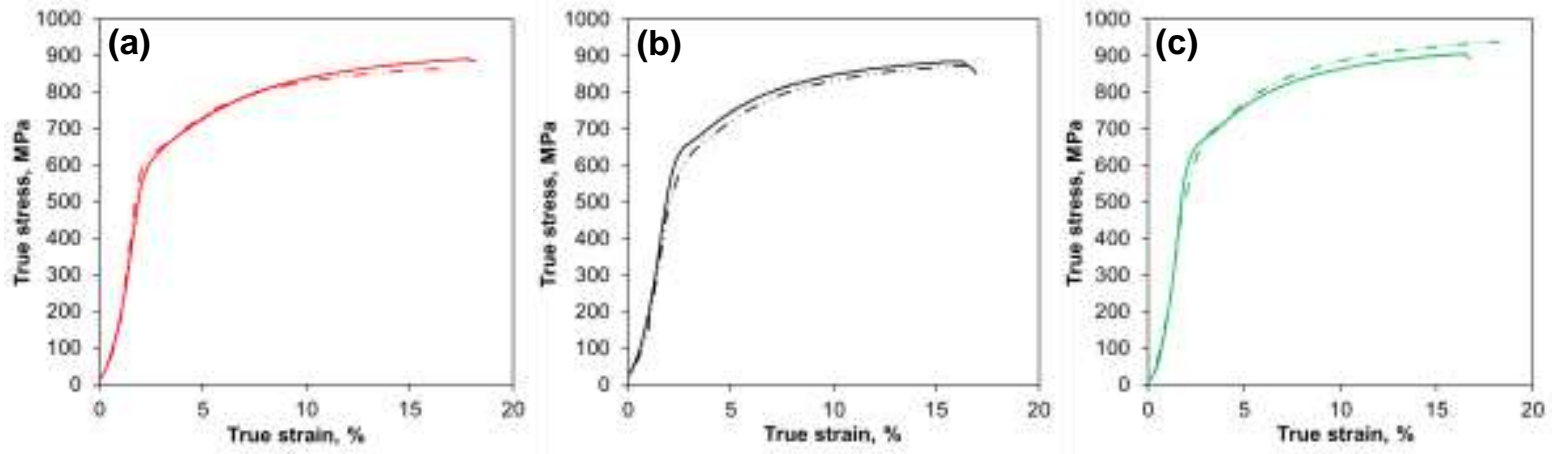

A 1.1 Compressive flow curves of two distinct reproducible tests for the 2024Al alloy compressed at RT and strain rates of (a) $10^{-3} \mathrm{~s}-1$, (b) $10^{-2} \mathrm{~s}^{-1}$, and (c) $10^{-1} \mathrm{~s}^{-1}$.
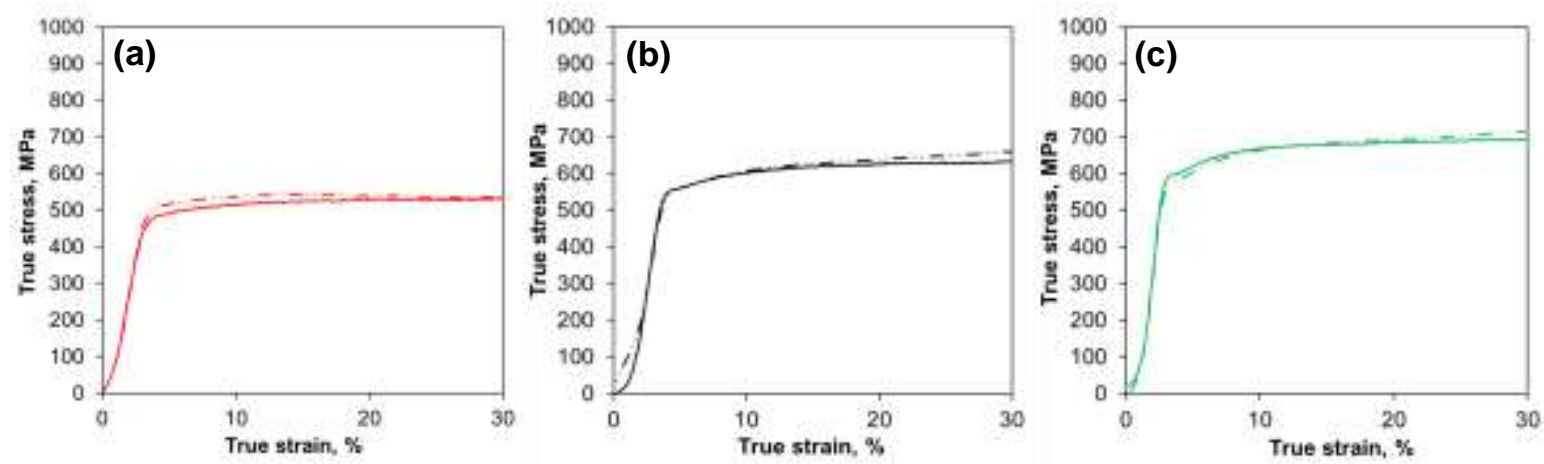

A 1.2 Compressive flow curves of two distinct reproducible tests for the 2024Al alloy compressed at $200^{\circ} \mathrm{C}$ and strain rates of (a) $10^{-3} \mathrm{~s}-1$, (b) $10^{-2} \mathrm{~s}^{-1}$, and (c) $10^{-1} \mathrm{~s}^{-1}$. 

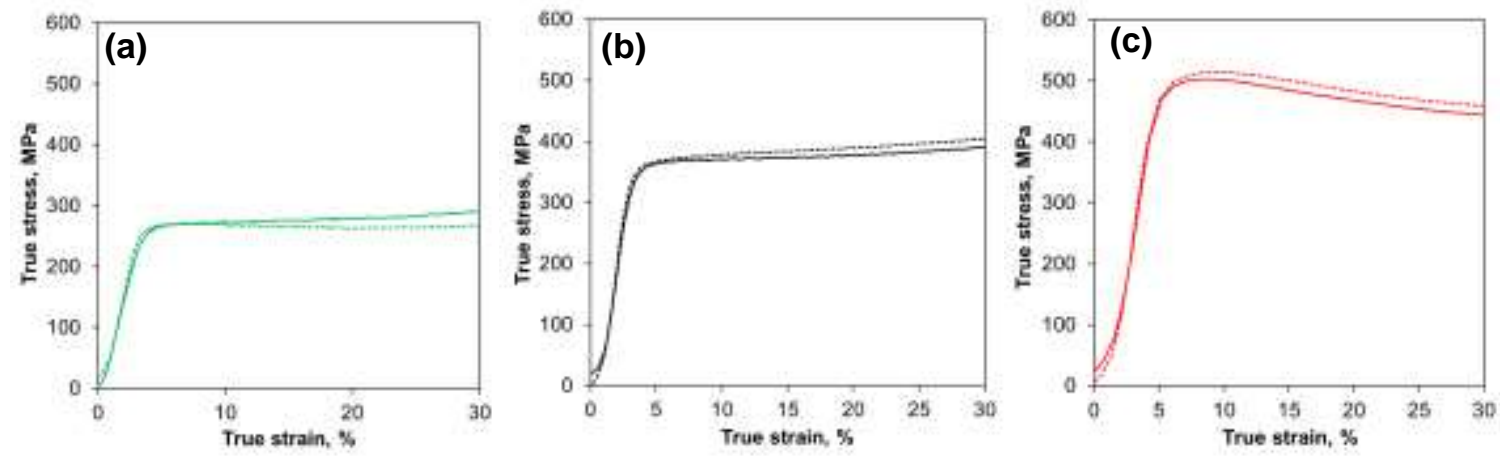

A 1.3 Compressive flow curves of two distinct reproducible tests for the 2024Al alloy compressed at $250^{\circ} \mathrm{C}$ and strain rates of (a) $10^{-3} \mathrm{~s}-1$, (b) $10^{-2} \mathrm{~s}^{-1}$, and (c) $10^{-1} \mathrm{~s}^{-1}$.
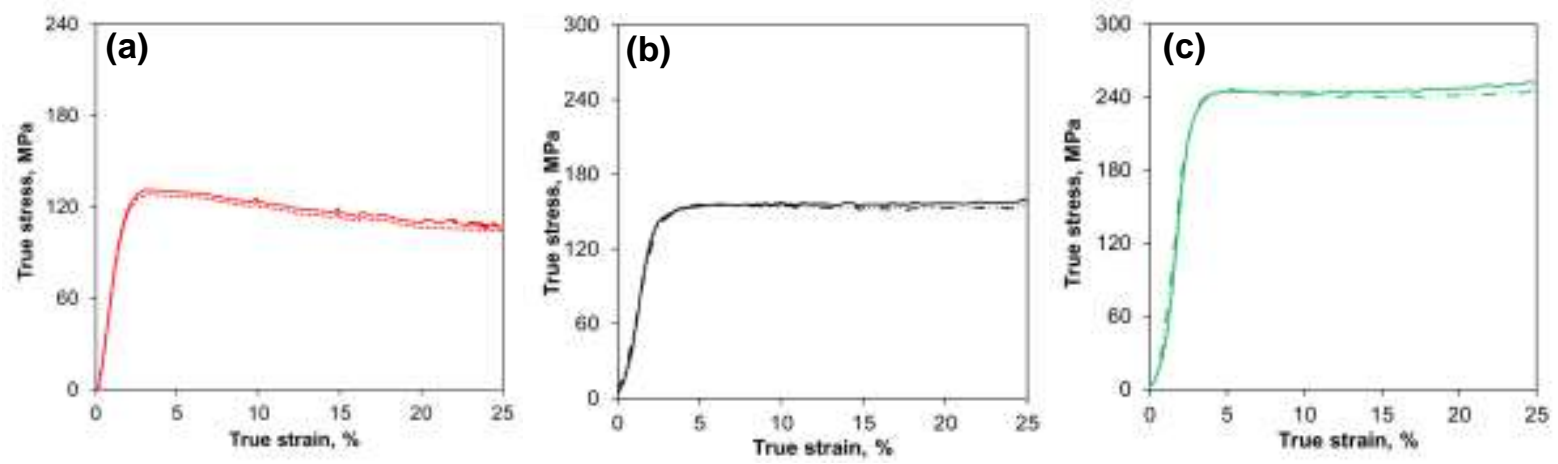

A 1.4 Compressive flow curves of two distinct reproducible tests for the 2024Al alloy compressed at $300^{\circ} \mathrm{C}$ and strain rates of (a) $10^{-3} \mathrm{~s}-1$, (b) $10^{-2} \mathrm{~s}^{-1}$, and (c) $10^{-1} \mathrm{~s}^{-1}$.
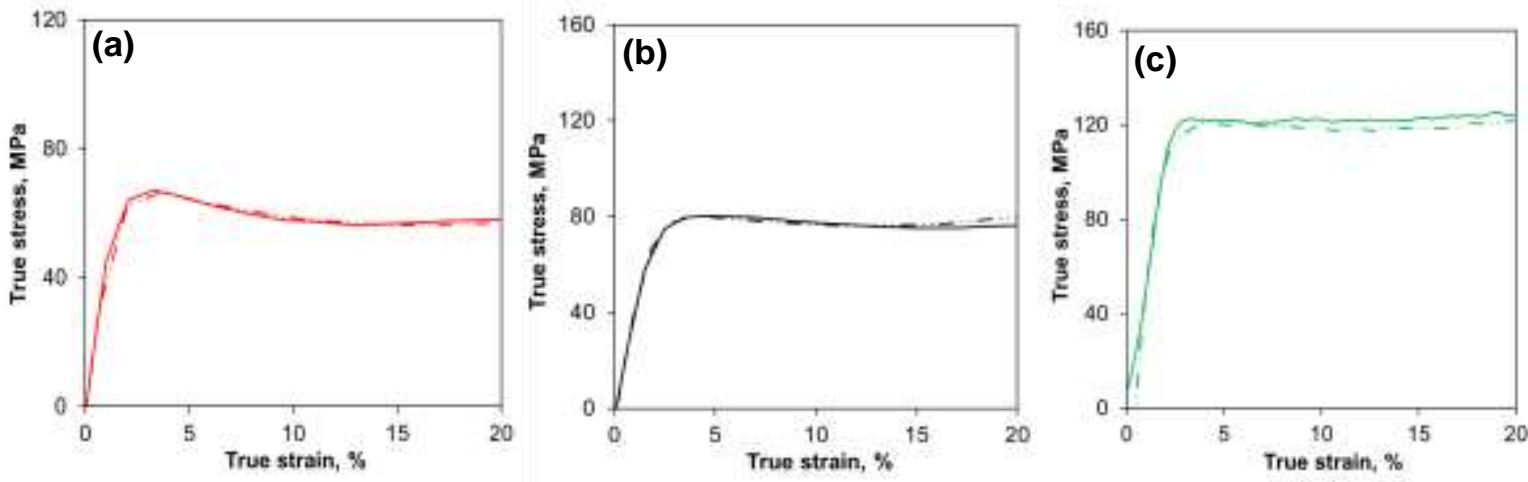

A 1.5 Compressive flow curves of two distinct reproducible tests for the 2024Al alloy compressed at $350^{\circ} \mathrm{C}$ and strain rates of (a) $10^{-3} \mathrm{~s}-1$, (b) $10^{-2} \mathrm{~s}^{-1}$, and (c) $10^{-1} \mathrm{~s}^{-1}$. 

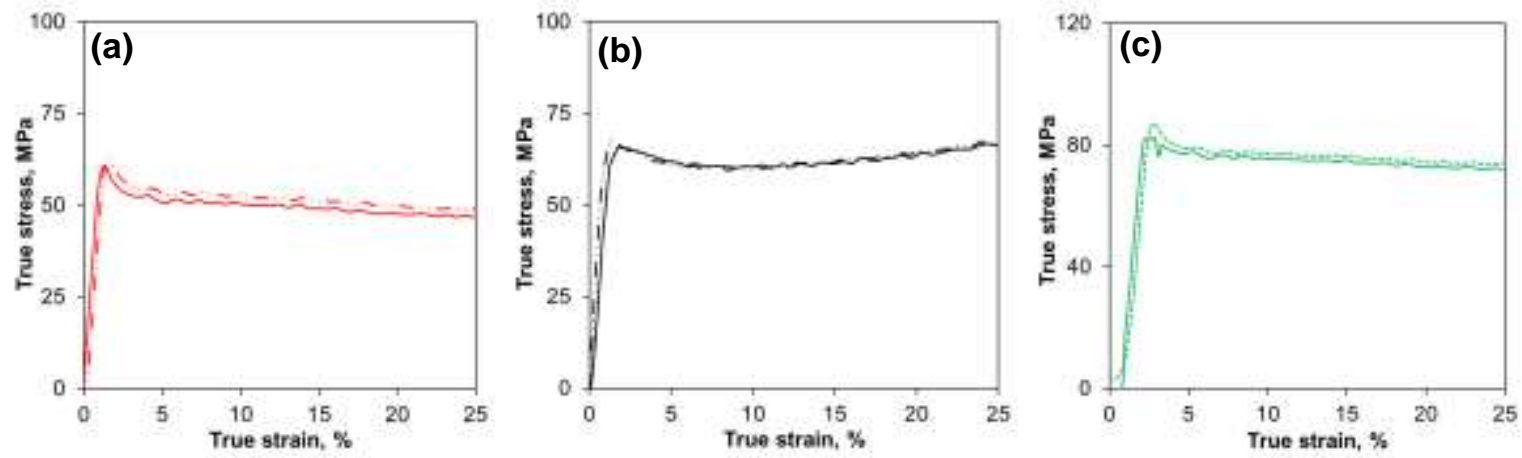

A 1.6 Compressive flow curves of two distinct reproducible tests for the 2024Al alloy compressed at $400^{\circ} \mathrm{C}$ and strain rates of (a) $10^{-3} \mathrm{~s}-1$, (b) $10^{-2} \mathrm{~s}^{-1}$, and (c) $10^{-1} \mathrm{~s}^{-1}$.
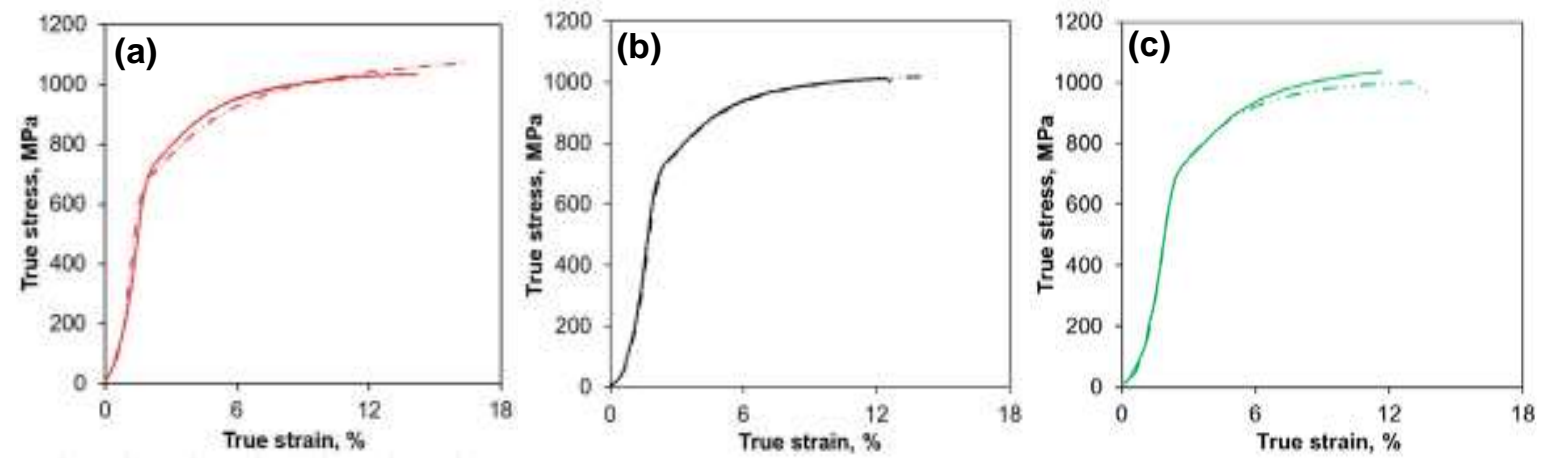

A 1.7 Compressive flow curves of two distinct reproducible tests for the $2.0 \mathrm{wt}$.\% CNT 2024Al composite compressed at RT and strain rates of (a) $10^{-3} \mathrm{~s}-1$, (b) $10^{-2} \mathrm{~s}^{-1}$, and (c) $10^{-1} \mathrm{~s}^{-1}$.
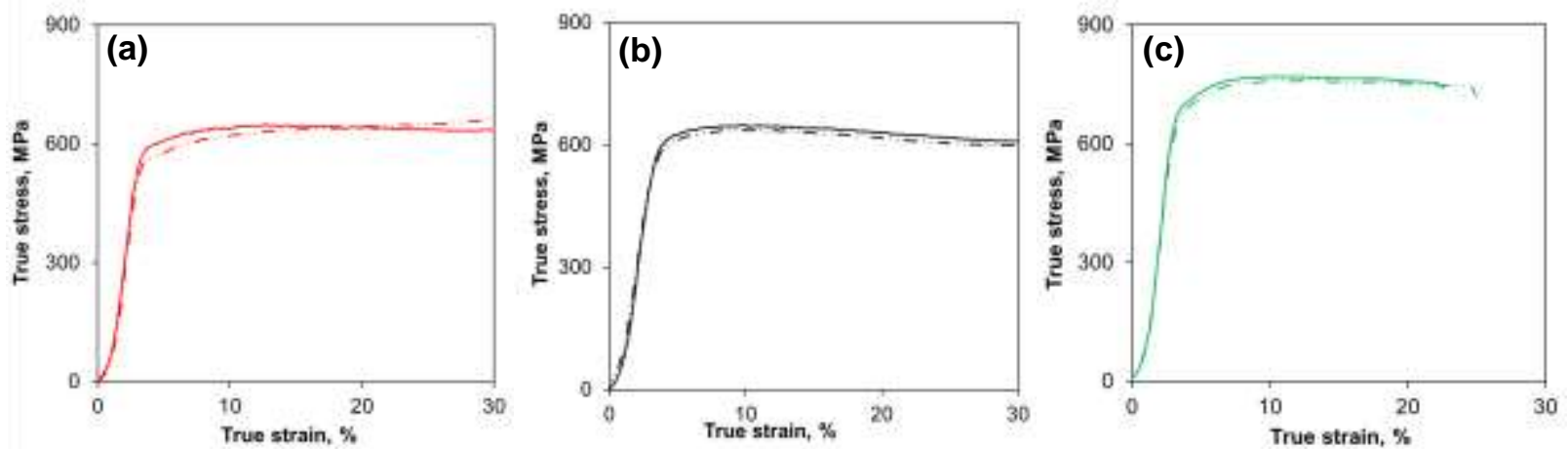

A 1.8 Compressive flow curves of two distinct reproducible tests for the $2.0 \mathrm{wt} . \%$ CNT $2024 \mathrm{Al}$ composite compressed at $200^{\circ} \mathrm{C}$ and strain rates of (a) $10^{-3} \mathrm{~s}-1$, (b) $10^{-2} \mathrm{~s}^{-1}$, and (c) $10^{-1} \mathrm{~s}^{-1}$. 

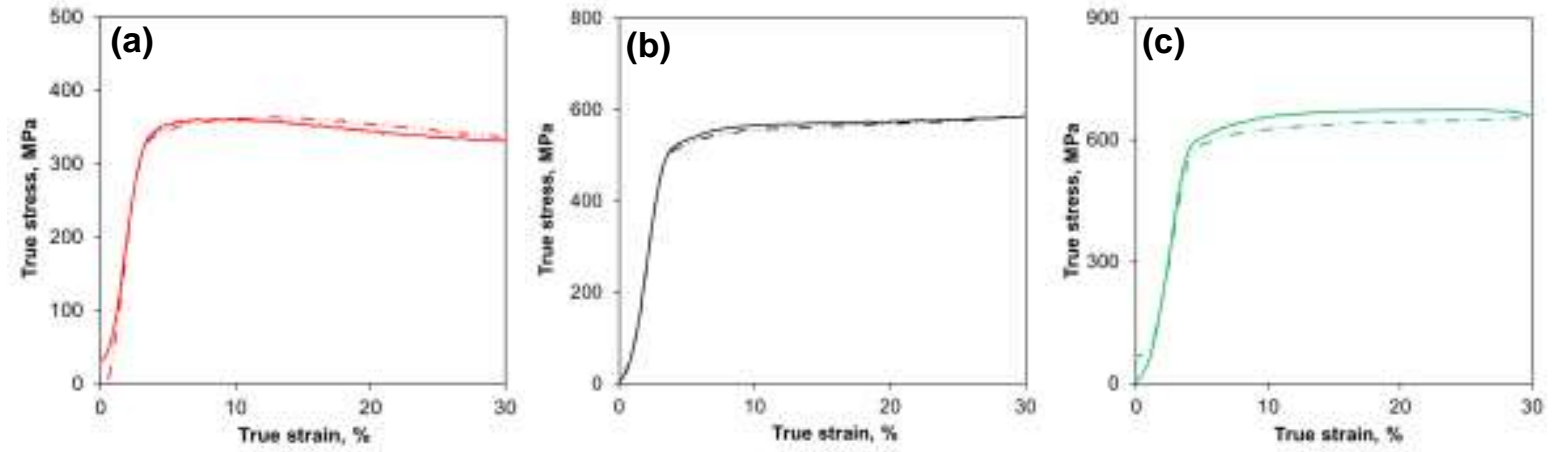

A 1.9 Compressive flow curves of two distinct reproducible tests for the $2.0 \mathrm{wt} . \%$ CNT 2024Al composite compressed at $250^{\circ} \mathrm{C}$ and strain rates of (a) $10^{-3} \mathrm{~s}-1$, (b) $10^{-2} \mathrm{~s}^{-1}$, and (c) $10^{-1} \mathrm{~s}^{-1}$.
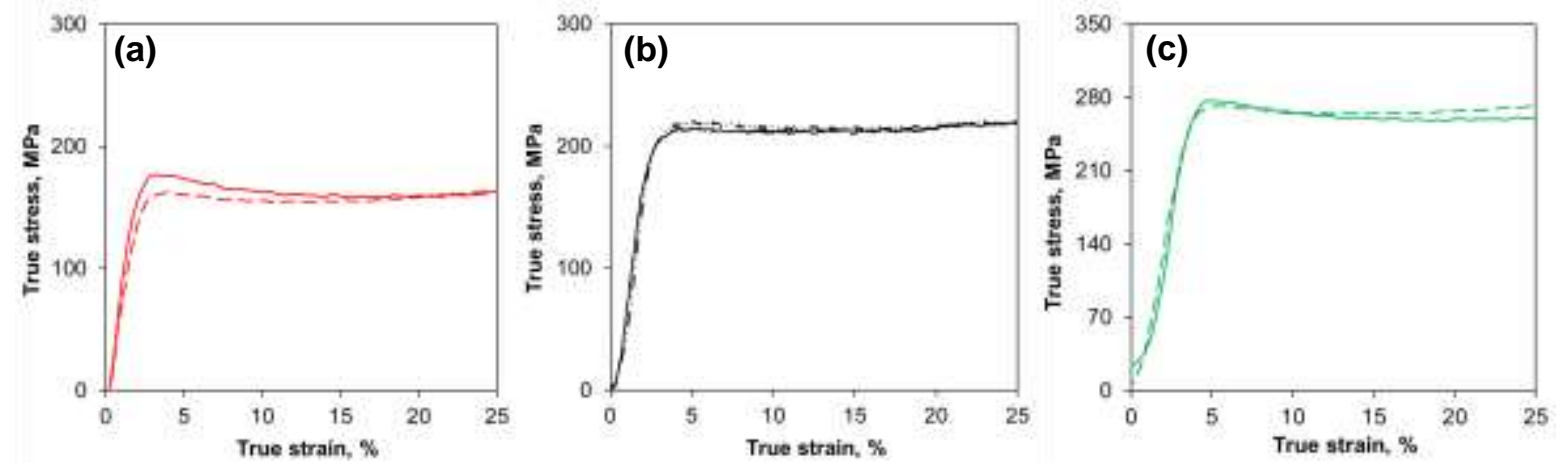

A 1.10 Compressive flow curves of two distinct reproducible tests for the 2.0 wt. \% CNT 2024Al composite compressed at $300^{\circ} \mathrm{C}$ and strain rates of (a) $10^{-3} \mathrm{~s}-1$, (b) $10^{-2} \mathrm{~s}^{-1}$, and (c) $10^{-1} \mathrm{~s}^{-1}$.
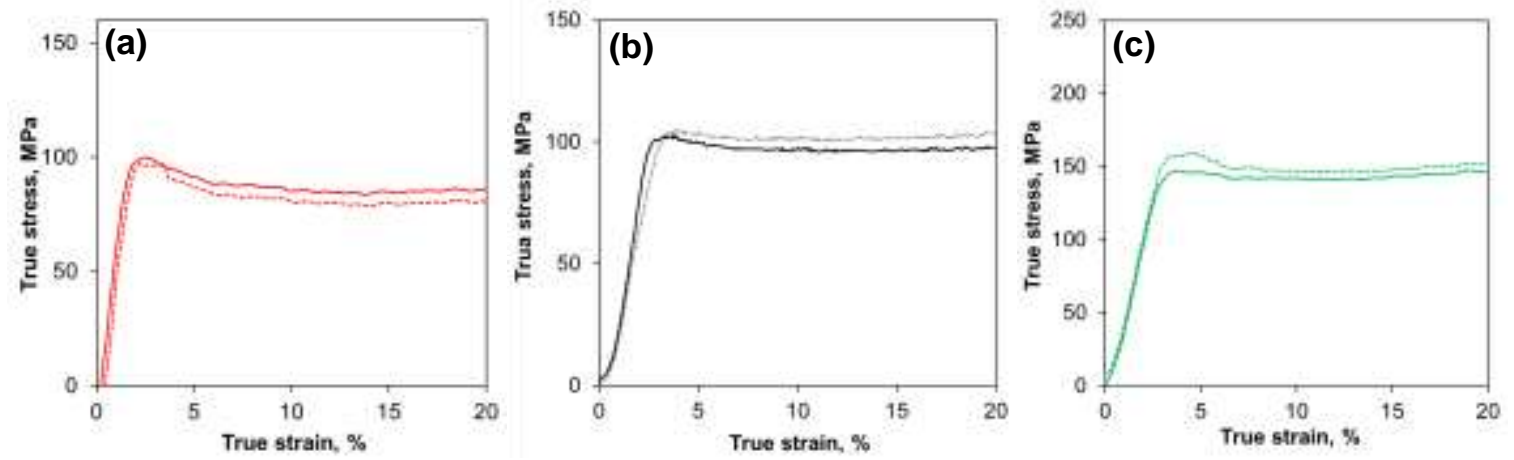

A 1.11 Compressive flow curves of two distinct reproducible tests for the $2.0 \mathrm{wt}$ \% CNT 2024Al composite compressed at $350^{\circ} \mathrm{C}$ and strain rates of (a) $10^{-3} \mathrm{~s}-1$, (b) $10^{-2} \mathrm{~s}^{-1}$, and (c) $10^{-1} \mathrm{~s}^{-1}$. 

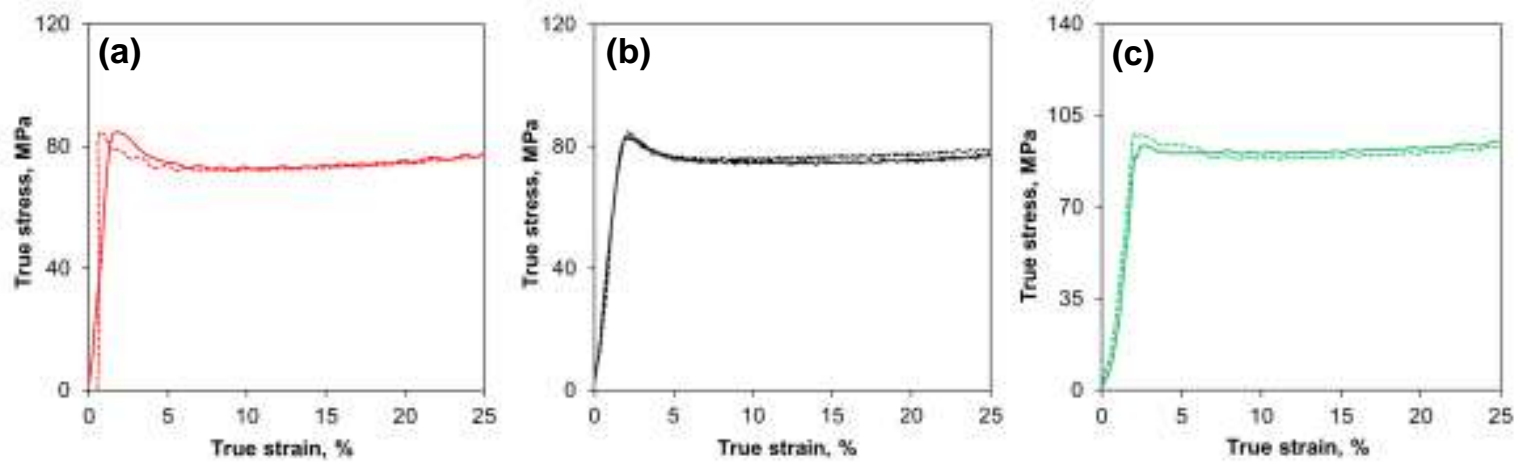

A 1.12 Compressive flow curves of two distinct reproducible tests for the $2.0 \mathrm{wt}$.\% CNT 2024Al composite compressed at $400^{\circ} \mathrm{C}$ and strain rates of (a) $10^{-3} \mathrm{~s}-1$, (b) $10^{-2} \mathrm{~s}^{-1}$, and (c) $10^{-1} \mathrm{~s}^{-1}$. 


\section{REFERENCES}

[1] McGlade C, Ekins P. The geographical distribution of fossil fuels unused when limiting global warming to $2^{\circ} \mathrm{C}$. Nature $2015 ; 517: 187-90$.

[2] Schaedler TA, Jacobsen AJ, Carter WB. Toward lighter, stiffer materials. Science 2013;341:1181-2.

[3] Nie JF, Zhu YM, Liu JZ, Fang XY. Periodic segregation of solute atoms in fully coherent twin boundaries. Science 2013;340:957-60.

[4] Schmale J, Shindell D, Von Schneidemesser E, Chabay I, Lawrence M. Air pollution: Clean up our skies. Nature 2014;515:335-7.

[5] Underwood E. Models predict longer, deeper US droughts. Science 2015;347:707.

[6] Chow EW, Heywood JB, Speth RL. Benefits of a higher octane standard gasoline for the US light-duty vehicle fleet. SAE Tech 2014;1961:1-18.

[7] Murray J, King D. Oil's tipping point has passed. Nature 2012;481:433-5.

[8] Joost WJ. Reducing vehicle weight and improving U.S. energy efficiency using integrated computational materials engineering. JOM 2012;64:1032-8.

[9] Elsayed A, Junko U, Katsuyoshi K. Application of rapid solidification powder metallurgy to the fabrication of high-strength, high-ductility $\mathrm{Mg}-\mathrm{Al}-\mathrm{Zn}-\mathrm{Ca}-\mathrm{La}$ alloy through hot extrusion. Acta Mater 2011;59:273-82.

[10] Hiroyuki W, Sawada T, Sasakura Y, Lkeo N, Mukai T. Microyielding and damping capacity in magnesium. Scripta Mater 2014;87:1-4.

[11] Stanford N, Atwell D, Barnett MR. The effect of Gd on the recrystallization, texture and deformation behavior of magnesium-based alloys. Acta Mater 2010;58:6773-83. 
[12] Kainer KU, Wendt J, Hantzsche K, Bohlen J, Yi SB, Letzig D. Development of the microstructure and texture of RE containing magnesium alloys during hot rolling. Mater Sci Forum 2010;654:580-5.

[13] Hirsch J, Al-Samman T. Superior light metals by texture engineering: optimized aluminum and magnesium alloys for automotive applications. Acta Mater 2013;61:81843.

[14] Samman TA, Li X. Sheet texture modification in magnesium-based alloys by selective rare earth alloying. Mater Sci Eng A 2011;528:3809-22.

[15] Wang FH, Dong J, Jiang YY, Ding WJ. Cyclic deformation and fatigue of extruded MgGd-Y magnesium alloy. Mater Sci Eng A 2013;561:403-10.

[16] Mohamed AM, Samuel FH, Al-kahtani S. Microstructure, tensile properties and fracture behavior of high temperature Al-Si-Mg-Cu cast alloys. Mater Sci Eng A 2013;577:64-72.

[17] Behabtu N, Young CC, Tsentalovich DE, Kleinerman O, Wang X, Anson W. Strong, light, multifunctional fibers of carbon nanotubes with ultrahigh conductivity. Science 2013;339:182-6.

[18] Ajayan PM, Tour JM. Materials science: nanotube composites. Nature 2007;447:1066-8.

[19] Yang C, Huang HF, De Los Reyes M, Yan L, Zhou XT, Xia T, Zhang DL. Microstructures and tensile properties of ultrafine-grained $\mathrm{Ni}-(1-3.5)$ wt $\% \mathrm{SiCNP}$ composites prepared by a powder metallurgy route. Acta Metall Sin 2015;28:809-16.

[20] Dong S, Zhou J, Hui D. A quantitative understanding on the mechanical behaviors of carbon nanotube reinforced nano/ultrafine-grained composites. Int $\mathrm{J}$ Mech Sci 2015;101:29-37. 
[21] Barai P, Weng GJ. The competition of grain size and porosity in the viscoplastic response of nanocrystalline solids. Int J Plasticity 2008;24:1380-410.

[22] Silvestre N, Faria B, Lopes JNC. Compressive behavior of CNT-reinforced aluminum composites using molecular dynamics. Compos Sci Tech 2014;90:16-24.

[23] Choi HJ, Kwon GB, Lee GY, Bae DH. Reinforcement with carbon nanotubes in aluminum matrix composites. Scripta Mater 2008;59:360-3.

[24] Bakshi SR, Keshri AK, Agarwal A. A comparison of mechanical and wear properties of plasma sprayed carbon nanotube reinforced aluminum composites at nano and macro scale. Mater Sci Eng A 2011;528:3375-84.

[25] Saheb N. Compressive behavior of spark plasma sintered CNT reinforced A12124 and Al6061 nanocomposites. Adv Mater Res 2013;652:33-7.

[26] Bustamante RP, Bustamante FP, Guel IE, Rodriguez CRS, Aquino JAM, Ramirez JMH. Characterization of Al2024-CNTs composites produced by mechanical alloying. Powder Technol 2011;212:390-6.

[27] Bustamante RP, Bustamante FP, Flores WA, Ramirez JMH. Al2024-CNTs composites by mechanical alloying. Microsc Microanal 2010;16:1256-7.

[28] Bustamante RP, Bustamante FP, Guel IE, Jimenez LL, Yoshida MM, Sanchez RM. Effect of milling time and CNT concentration on hardness of CNT/Al2024 composites produced by mechanical alloying. Mater Charact 2013;75:13-9.

[29] Hao XN, Zhang HP, Zheng RX, Zhang YT, Ameyama K, Ma CL. Effect of mechanical alloying time and rotation speed on evolution of CNTs/Al-2024 composite powders. J Nonferr Metal Soc China 2014;24:2380-6. 
[30] Qi WH, Wang MP. Size and shape dependent lattice parameters of metallic nanoparticles. J Nanopart Res 2005;7:51-7.

[31] Mirza FA, Chen DL. Fatigue of lightweight magnesium alloys. Aerospace Materials Handbook, CRC Press, Taylor \& Francis, New York 2013;647-98.

[32] Eliezer D, Aghion E, Froes, FH. Magnesium science, technology and applications. Adv Perform Mater 1998;5:201-12.

[33] Pollock TM. Weight loss with magnesium alloys. Science 2010;328:986-7.

[34] Mordike BL, Ebert T. Magnesium: Properties applications potential. Mater Sci Eng A 2001;302:37-45.

[35] Blawert C, Hort N, Kainer KU. Automotive applications of magnesium and its alloys. Trans Indian Inst Met 2004;57:397-408.

[36] United States Automotive Materials Partnership (USAMP). Magnesium vision 2020: A North American automotive strategic vision for magnesium 2006.

[37] Edwards JD, Frary FC, Jeffries Z. The Aluminum industry, McGraw-Hill, New York 1930.

[38] Howatson AM, Lund PG, Todd JD. Engineering tables and data, Chapman \& Hall, Manchester, 1992.

[39] Kainer KU. Magnesium alloys and technology. Wiley-VCH, Cambridge 2003.

[40] K. Louisville K. The ABC's of aluminum. Reynolds Metals Company, Virginia 1953.

[41] Kaufman JG. Introduction to Aluminum alloys and tempers. ASM International, Ohio 2000.

[42] Tenckhoff E. Deformation mechanisms, texture, and anisotropy in zirconium and zircaloy. American Society for Testing and Materials, Philadelphia 1988. 
[43] Yoo MH, Morris JR, Ho KM, Agnew SR. Nonbasal deformation modes of hcp metals and alloys: Role of dislocation source and mobility. Metall Mater Trans A 2002;33:81322.

[44] Callister Jr WD, Rethwisch DG. Materials science and engineering: An introduction. John Wiley and Sons Inc, York 2008.

[45] Yoo MH. Slip, Twinning, and fracture in hexagonal close-packed metals. Metall Mater Trans A 1981;12:409-18.

[46] Jiang J, Godfrey A, Liu W, Liu Q. Microtexture evolution vie deformation twinning and slip during compression of magnesium alloy AZ31. Mater Sci Eng A 2008;483:576-9.

[47] Zhu YT, Wu XL, Liao XZ, Narayan J, Kecskes LJ, Mathaudhu SN. Dislocation-twin interactions in nanocrystalline fcc metals. Acta Mater 2011;59:812-21.

[48] Park SH, Hong SG, Lee JH, Lee CS. Multiple twinning modes in rolled Mg-3Al-1Zn alloy and their selection mechanism. Mater Sci Eng A 2012;532:401-6.

[49] Xin Y, Wang M, Zeng Z, Nie M, Liu Q. Strengthening and toughening of magnesium alloy by $\{10-12\}$ extension twins. Scripta Mater. 2012;66:25-8.

[50] Gharghouri MA, Weatherly GC, Embury JD, Root J. Study of the mechanical properties of Mg- 7.7 at.\% Al by in-situneutron diffraction. Phil. Mag. A 1999;79:1671-95.

[51] Godet S, Jiang L, Luo AA, Jonas JJ. Use of Schmid factors to select extension twin variants in extruded magnesium alloy tubes. Scripta Mater 2006;55:1055-8.

[52] El Kadiri H, Kapil J, Oppedal AL, Hector Jr LG, Agnew SR, Cherkaoui M, Vogel SC. The effect of twin-twin interactions on the nucleation and propagation of $\{10-12\}$ twinning in magnesium. Acta Mater 2013;61:3549-63. 
[53] Wang H, Wu PD, Wang J, Tome CN. A crystal plasticity model for hexagonal close packed (HCP) crystals including twinning and de-twinning mechanisms. Inter J Plasticity 2013;49:36-52.

[54] Beyerlein IJ, Wang J, Barnett MR, Tome CN. Double twinning mechanisms in magnesium alloys via dissociation of lattice dislocations. Proc R Soc A 2012;1098:1-25.

[55] Ma Q, El Kadiri H, Oppedal AL, Baird JC, Horstemeyer MF, Cherkaoui M. Twinning and double twinning upon compression of prismatic textures in an AM30 magnesium alloy. Scripta Mater 2011;64:813-6.

[56] Castor SB, Hedrick JB. Rare elements. Indust Miner 2006;7:769-92.

[57] Emsley J. Nature's building blocks: An A-Z guide to the elements. Oxford University Press, Oxford 2001.

[58] Lee BH, Park SH, Hong SG, Park KT, Lee CS. Role of initial texture on the plastic anisotropy of $\mathrm{Mg}-3 \mathrm{Al}-1 \mathrm{Zn}$ alloy at various temperatures. Mater Sci Eng A 2011;528:1162-72.

[59] Perez-Prado MT, Del Valle JA, Ruano OA. Grain refinement of Mg-Al-Zn alloys via accumulative roll bonding. Scripta Mater. 2004;51:1093-7.

[60] Ball EA, Prangnell PB. Tensile-compressive yield asymmetries in high strength wrought magnesium alloys. Scripta Metall. Mater. 1994;31:111-6.

[61] Mackenzie LWF, Pekguleryuz MO. The recrystallization and texture of magnesiumzinc-cerium alloys. Scripta Mater 2008;59:665-8.

[62] Senn JW, Agnew SR. Texture randomization of magnesium alloys containing rare earth elements. Proc TMS 2008:153-8. 
[63] Liu K, Zhang J, Rokhlin LL, Elkin FM, Tang D, Meng J. Microstructures and mechanical properties of extruded $\mathrm{Mg}-8 \mathrm{Gd}-0.4 \mathrm{Zr}$ alloys containing $\mathrm{Zn}$. Mater Sci Eng A 2009;505:13-9.

[64] Liu XB, Chen RS, Han EH. Effects of ageing treatment on microstructures and properties of Mg-Gd-Y-Zr alloys with and without Zn additions. J Alloys Comp 2008;465:232-8.

[65] Al-Samman, T. Modification of texture and microstructure of magnesium alloy extrusions by particle-stimulated recrystallization. Mater Sci Eng A 2013;560:561-6.

[66] Robson JD, Twier AM, Lorimer GW, Rogers P. Effect of extrusion conditions on microstructure, texture, and yield asymmetry in Mg-6Y-7Gd-0.5\% Zr alloy. Mater Sci Eng A 2011;528:7247:56.

[67] Bohlen J, Nurnberg MR., Senn JW, Letzig D, Agnew SR. The texture and anisotropy of magnesium-zinc-rare earth alloy sheets. Acta Mater 2007;55:2101-12.

[68] Stanford N, Barnett MR. The origin of "Rare Earth" texture development in extruded Mg-based alloys and its effect on tensile ductility. Mater Sci Eng A 2008;496:399-408.

[69] Nie JF, Muddle BC. Characterization of strengthening precipitate phases in a $\mathrm{Mg}-\mathrm{Y}-\mathrm{Nd}$ alloy. Acta Mater 2000;48:1691-1703.

[70] Hantzsche K, Bohlen J, Wendt J, Kainer KU, Yi SB, Letzig D. Effect of rare earth additions on microstructure and texture development of magnesium alloy sheets. Scripta Mater 2010;63:725-30.

[71] Stanford N. Micro-alloying Mg with $\mathrm{Y}, \mathrm{Ce}, \mathrm{Gd}$ and $\mathrm{La}$ for texture modification-a comparative study. Mater Sci Eng A 2010;527:2669-77.

[72] Potzies C, Kainer KU. Fatigue of magnesium alloys. Adv Eng Mater2004;6:281-9. 
[73] Yang F, Lv F, Yang XM, Li SX, Zhang ZF, Wang QD. Enhanced very high cycle fatigue performance of extruded $\mathrm{Mg}-12 \mathrm{Gd}-3 \mathrm{Y}-0.5 \mathrm{Zr}$ magnesium alloy. Mater Sci Eng A 2011;528:2231-8.

[74] Maier P, Tober G, Mendis CL, Müller S, Hort N. Influence of Nd in extruded Mg-10Gd base alloys on fatigue strength, Adv Mater Res 2014;783:419-24.

[75] Mirza FA, Chen DL, Li DJ, Zeng XQ. Low cycle fatigue of a rare-earth containing extruded magnesium alloy. Mater Sci Eng A 2013;575:65-73.

[76] Mirza FA, Chen DL, Li DJ, Zeng XQ. Low cycle fatigue of an extruded Mg-3Nd-0.2Zn0.5Zr magnesium alloy. Mater Des 2014;64:63-73.

[77] Begum S, Chen DL, Xu S, Luo AA. Low cycle fatigue properties of an extruded AZ31 magnesium alloy. Int J Fatigue 2009;31:726-35.

[78] Min JY, Lin JP. Anelastic behavior and phenomenological modeling of Mg ZEK100-O alloy sheet under cyclic tensile loading-unloading. Mater Sci Eng A 2013;561:174-82.

[79] Antoniswamy AR, Carpenter AJ, Carter JT, Hector Jr LG, Taleff EM. Forming-Limit Diagrams for Magnesium AZ31B and ZEK100 Alloy Sheets at Elevated Temperatures. J Mater Eng. Perform. 2013;22:3389-97.

[80] Min J, Lin J, Li J. Forming limits of Mg alloy ZEK100 sheet in preform annealing process. Mater Des 2014;53:947-53.

[81] Niu XP, Skszek T, Fabischek M, Zak A. Low temperature warm forming of magnesium ZEK100 sheets for automotive applications. Adv Mater Res 2014;783:431-6.

[82] Kurukuri S, Worswick M, Bardelcik A, Mishra R, Carter J. Constitutive behavior of commercial grade ZEK100 magnesium alloy sheet over a wide range of strain rates. Metall Mater Trans A 2014;45:3321-37. 
[83] Aslam I, Li B, McClelland Z, Horstemeyer SJ, Ma Q, Wang PT, Horstemeyer MF. Three-point bending behavior of a ZEK100 Mg alloy at room temperature. Mater Sci Eng A 2014;590:168-73.

[84] Tasis D, Tagmatarchis N, Bianco A, Prato M. Chemistry of carbon nanotubes. Chem Rev 2006;106:1105-36.

[85] Dai H. Carbon nanotubes: synthesis, integration and properties. Acc Chem Res2002;35:1035-44.

[86] Bakshi SR, Lahiri D, Agarwal A. Carbon nanotube reinforced metal matrix composites a review. Int Mater Rev 2010;55:-41-64.

[87] Xie S, Li W, Pan Z, Chang B, Sun L. Mechanical and physical properties on carbon nanotube. J Phys Chem Solid 2000;61:1153-1158.

[88] Huang Y, Quyang Q, Zhang D, Zhu J, Li R, Yu H. Carbon materials reinforced aluminum composites: A review. Acta Metall. Sin. 2014;27:775-86.

[89] Bakshi SR, Singh V, Seal S, Agarwal A. Aluminum composite reinforced with multiwalled carbon nanotubes from plasma spraying of spray dried powders. Surf Coat Technol 2009;203:1544-54.

[90] Salimi S, Izadi H, Gerlich A. Fabrication of an aluminum-carbon nanotube metal matrix composite by accumulative roll-bonding. J Mater Sci 2011;46:409-15.

[91] Fan G, Yu Z, Tan Z, Li Z, Zhang D. Evolution, control, and effects of interface in CNT/Al composite: a review. Acta Metall Sin 2014;27:839-43.

[92] Esawi AMK, Morsi K, Sayed A, Gawad AA, Borah P. Fabrication and properties of dispersed carbon nanotube-aluminum composites. Mater Sci Eng A 2009;508:167-73. 
[93] Poirier D, Gauvin R, Drew RAL. Structural characterization of a mechanically milled carbon nanotube/aluminum mixture. Compos A 2009;40:1482-9.

[94] Michio I, Kaburagi Y, Hishiyama Y. Thermal Management Material: Graphite. Adv. Eng. Mater. 2014;16:494-506.

[95] Liu ZY, Xiao BL, Wang WG, Ma ZY, Singly dispersed carbon nanotube/aluminum composites fabricated by powder metallurgy combined with friction stir processing. Carbon 2012;50:1843-52.

[96] Perez BR, Bustamante F, Estrada GI, Jimenez LL, Yoshida MM, Sanchez RM. Effect of milling time and CNT concentration on hardness of CNT/Al2024 composites produced by mechanical alloying. Mater. Character. 2013;75:13-9.

[97] Bakshi SR, Agarwal A. An analysis of the factors affecting strengthening in carbon nanotube reinforced aluminum composites. Carbon 2011;49:533-44.

[98] Min J, Lin J, Li J. Forming limits of Mg alloy ZEK100 sheet in preform annealing process. Mater Des 2014;53:947-953.

[99] Wang YN, Huang JC. Texture analysis in hexagonal materials. Mater Chem Phys $2003 ; 81: 11-26$.

[100] Yi S, Zaefferer S, Brokmeier H. Mechanical behaviour and microstructural evolution of magnesium alloy AZ31 in tension at different temperatures. Mater Sci Eng A 2006;424:275-281.

[101] Chino Y, Kimura K, Mabuchi M. Twinning behavior and deformation mechanisms of extruded AZ31 Mg alloy. Mater Sci Eng A 2008;486;481-8.

[102] Yu Q, Wang J, Jiang Y, Mccabe RJ, Li N, Tome CN. Twin-twin interactions in magnesium. Acta Mater 2014;77:28-42. 
[103] Yu Q, Wang J, Jiang Y, Mccabe RJ, Tome CN. Co-zone $\{10-12\}$ twin interaction in magnesium single crystal. Mater Res Lett 2013:2;82:88.

[104] Kwon H, Nakano H, Mabuchi M, Chino Y. Twinning behaviour of AZ31 Mg alloy alternately compressed in two orthogonal directions. Philos Mag 2014;94:3960-3977.

[105] Meng L, Yang P, Xie Q, Mao W. Analyses on compression twins in magnesium. Mater Trans 2008;49:710-714.

[106] Wang J, Zeng Z, Weinberger CR., Zhang Z, Zhu T, Mao SX. In situ atomic-scale observation of twinning dominated deformation in nano-scale body-centered cubic tungsten. Nat Mater 2015;14:594-600.

[107] Koike J. Enhanced deformation mechanisms by anisotropic plasticity in polycrystalline $\mathrm{Mg}$ alloys at RT. Metall Mater Trans A 2005;36:1689-96.

[108] Wang F, Agnew SR. Dislocation transmutation by tension twinning in magnesium alloy AZ31. Int J Plasticity 2016;81:63-86.

[109] Avedesian MM, Baker H. Magnesium and magnesium alloys. ASM International 1999.

[110] Hong S, Park SH, Lee CS. Role of $\{10-12\}$ twinning characteristics in the deformation behavior of a polycrystalline magnesium alloy. Acta Mater 2010;58:5873-85.

[111] Barnett M, Keshavarz Z, Beer A, Atwall D. Influence of grain size on the compressive deformation of wrought Mg-3Al-1Zn. Acta Mater 2004;52:5093-103.

[112] Martin G, Sinclair CW, Lebensohn RA. Microscale plastic strain heterogeneity in slip dominated deformation of magnesium alloy containing rare earth. Mater Sci Eng A 2014;603:37-51. 
[113] Hantzsche K, Wendt J, Kainer KU, Bohlen J, Letzig D. Mg sheet: the effect of process parameters and alloy composition on texture and mechanical properties . JOM 2009;61:38-42.

[114] Aslam I, Li B, McClelland Z, Horstemeyer SJ, Ma Q, Wang PT, Horstemeyer MF. Three-point bending behavior of a ZEK100 Mg alloy at room temperature. Mater Sci Eng A 2014;590:168-73.

[115] Hashemi AAN, Clark JB. The Mg-Nd system (Magnesium-Neodymium). J Phase Equilib 1988;9:618-23.

[116] Hashemi AAN. Phase diagrams of binary magnesium alloys. ASM Inter, Metals Park, Ohio 1988;370.

[117] Wu L, Jain A, Brown DW, Stoica GM, Agnew SR, Clausen B, Fielden D, Liaw PK. Twinning-detwinning behavior during the strain-controlled low-cycle fatigue testing of a wrought magnesium alloy, ZK60A. Acta Mater 2008;56:688-95.

[118] Gao L, Chen RS, Han EH. Effects of rare-earth elements Gd and Y on the solid solution strengthening of Mg alloys. J Alloy Compd 2009;481:379-84.

[119] Humphreys FJ. A unified theory of recovery, recrystallization and grain growth, based on the stability and growth of cellular microstructures-II. The effect of second-phase particles. Acta Mater 1997; 45:5031-9.

[120] Begum S, Chen DL, Xu S, Luo AA. Strain-controlled low-cycle fatigue properties of a newly developed extruded magnesium alloy. Metall Materials Trans A 2008;39:3014-26.

[121] Lin XZ, Chen DL. Strain controlled cyclic deformation behavior of an extruded magnesium alloy. Mater Sci Eng A 2008;496:106-13. 
[122] Jordon JB, Gibson JB, Horstmeyer MF, Kadiri HE, Luo AA. Effect of twinning, slip, and inclusions on the fatigue anisotropy of extrusion-textured AZ61 magnesium alloy. Mater Sci Eng A 2011;528:6860-71.

[123] Yin SW, Wang CH, Diao YD, Wu SD, Li SX. Influence of grain size and texture on the yield asymmetry of Mg-3Al-1Zn alloy. J Mater Sci Tech 2011;27:29-34.

[124] Mokdad F, Chen DL. Strain controlled low cycle fatigue properties of a rare-earth containing ZEK100 magnesium alloy. Mater Des 2015;67:274-83.

[125] Mokdad F, Chen DL. Cyclic deformation and anelastic behavior of ZEK100 magnesium alloy: Effect of strain ratio. Mater Sci Eng A 2015;640:243-58.

[126] Barnett MR. A Taylor model based description of the proof stress of magnesium AZ31 during hot working. Metall Mater Transa A 2003;34:1799-1806.

[127] Christ HJ, Mughrabi H. Cyclic stress-strain response and microstructure under variable amplitude loading. Fract Eng Mater Struct 1996;19:335-48.

[128] Patel HA, Chen DL, Bhole SD, Sadayappan K. Cyclic deformation and twinning in a semi-solid processed AZ91D magnesium alloy. Mater Sci Eng A 2010;528:208-19.

[129] Feltner CE, Laird C. Cyclis stress-strain response of FCC metals and alloys - II Dislocation structures. Acta Metall 1967;15:1633-1653

[130] Dieter GE. Mechanical Metallurgy. SI metric ed, McGraw-Hill Inc, New York 1986.

[131] Ke JC, Lin CH. Sensitivity analysis of machine repair problems in manufacturing systems with service interruptions. Appl Math Model 2008; 32:2087-2105.

[132] Q. Lin Q. Quantifying and minimizing systematic and random errors in X-ray microtomography based volume measurements. Computers \& Geosciences 2015;77:1-7. 
[133] Cohen J, Cohen P, West SG, Aiken LS. Applied multiple regression/correlation analysis for the behavioral sciences. Routledge 2013.

[134] Saltelli A, Ratto M, Andres T, Campolongp F, Cariboni J, Gatelli D, Saisana M, Tarantola S. Global Sensitivity Analysis-The Primer. Wiley-Interscience, New York 2008.

[135] Zhang Z, Chen DL. Consideration of Orowan strengthening effect in particulatereinforced metal matrix nanocomposites: a model for predicting their yield strength. Scripta Mater 2006;54:1321-26.

[136] Fan G, Xu R, Tan Z, Zhang D, Li Z. Development of flake powder metallurgy in fabricating metal matrix composites: a review. Acta Metall Sin 2014;27:806-15.

[137] Suarez S, Moore ER, Lechthaler B, Mucklich F. Grain growth analysis of multiwalled carbon nanotube-reinforced bulk Ni composites. Carbon 2014;70:173-8.

[138] Choi HJ, Min BH, Shin JH, Bae DH. Strengthening in nanostructured 2024 aluminum alloy and its composites containing carbon nanotubes. Compos Part A 2011;42:1438-44.

[139] Williamson GK, Hall WH. X-ray line broadening from filed aluminum and wolfram. Acta Metall 1953;1:22-31.

[140] Cullity BD, Stock SR. Elements of X-Ray Diffraction. $3^{\text {rd }}$ Ed, Prentice Hall, New Jersey 2001.

[141] Vijay C, Song B, Casem D. Dynamic behavior of materials. Springer Science \& Business Media, New York 2012.

[142] Jafari M, Enayati MH, Abassi MH, Karimzadeh F. Thermal stability and structural changes during heat treatment of nanostructured Al2024 alloy. J Alloy Compd 2009;478:260-4. 
[143] Qi WH, Wang MP. Size and shape dependent lattice parameters of metallic nanoparticles. J Nanopart Res 2005;7:51-7.

[144] Courtney TH. Mechanical behavior of materials. Mc Graw-Hill, New York 1990.

[145] Randle V, Engler O. Introduction to texture analysis: macrotexture, microtexture and orientation mapping. CRC Press, New York 2000.

[146] Wenk HR, Van Houtte P. Texture and anisotropy. Rep Prog Phys 2004;67:1367-428.

[147] Kocks UF, Tome CN, Wenk HR. Texture and anisotropy: preferred orientations and their effect of material properties. Cambridge University Press, United Kingdom 2000.

[148] Habibi MK, Paramsothy M, Hamouda AMS, Gupta M. Using integrated hybrid (Al + CNT) reinforcement to simultaneously enhance strength and ductility of magnesium. Compos Sci Technol 2011;71:734-41.

[149] Engler O, Hirsch J, Lucke K. Texture development in Al-1.8wt.\% Cu depending on the precipitation state - I. Rolling textures. Acta Metall Mater 1989;37:2743-53.

[150] Engler O, Hirsch J, Lucke K. Texture development in Al-1.8 wt.\% Cu depending on the precipitation state - II. Recrystallization textures. Acta Metall Mater 1994;43:121-38.

[151] Miller WS, Humphreys FJ. Strengthening mechanisms in particulate metal matrix composites. Scripta Mater 1991;25:33-38.

[152] Park JG, Keum DH, Lee YH. Strengthening mechanisms in carbon nanotube-reinforced aluminum composites. Carbon 2015;95:690-8.

[153] Mokdad F, Chen DL, Liu ZY, Xiao BL, Ni DR, Ma ZY. Deformation and strengthening mechanisms of a carbon nanotube reinforced aluminum composite. Carbon 2016;104:6477. 
[154] Kim KT, Eckert J, Menzel SB, Gemming T, Hong SH. Grain refinement assisted strengthening of carbon nanotube reinforced copper matrix nanocomposites. Appl Phys Lett 2008;92:121901.

[155] Sandstrom R, Lehtinen B, Hedman E, Groza I, Karlsson S. Subgrain growth in Al and Al-1\% Mn during annealing. J Mater Sci 1978;13:1229-42.

[156] Cottrell AH. Theory of dislocations. Progress in metal physics. Proc Metal Phys $1953 ; 4: 251-5$.

[157] Kim WJ, Yu YJ. The effect of the addition of multiwalled carbon nanotubes on the uniform distribution of TiC nanoparticles in aluminum nanocomposites. Scripta Mater 2014;72:25-8.

[158] Nes E, Ryum N, Hunderi O. On the Zener drag. Acta Metall 1985;33:11-22.

[159] Rios PR, Siciliano Jr F, Sandim HRZ, Plaut RL, Padilha AF. Nucleation and growth during recrystallization. Mater Res 2005;8 :225-38.

[160] George R, Kashyap KT, Rahul R, Yamdagni S. Strengthening in carbon nanotube/aluminum (CNT/Al) composites. Scripta Mater 2005;53:1159-63.

[161] Kelly A, Tyson WR. Tensile properties of fibre reinforced metals. J Mech Phys Solids 1965;13:329-50.

[162] Kwon H, Estili M, Takagi K, Miyazaki T, Kawasaki A. Combination of hot extrusion and spark plasma sintering for producing carbon nanotube reinforced aluminum matrix composites. Carbon 2009;47:570-7.

[163] Sellars CM, McTegart WJ. On the mechanism of hot deformation. Acta Metall $1966 ; 14: 1136-8$. 
[164] McQueen HJ, Ryan ND. Constitutive analysis in hot working. Mater Sci Eng A 2002;322:43-6.

[165] Zhang MJ, Li FG, Wang SY, Liu CY. Characterization of hot deformation behavior of P/M nickel-base superalloy using processing map and activation energy. Mater Sci Eng A 2010;527:6771-9.

[166] Tahreen N, Zhang DF, Pan FS, Jiang XQ, Li DY, Chen DL. Hot deformation and processing map of an as-extruded $\mathrm{Mg}-\mathrm{Zn}-\mathrm{Mn}-\mathrm{Y}$ alloy containing I and $\mathrm{W}$ phases. Mater Des 2015;87:245-55.

[167] Malas JC, Venugopal S, Seshacharyulu. Effect of microstructural complexity on the hot deformation behavior of aluminum alloy 2024. Mater Sci Eng A 2004;368:41-7.

[168] Wang S, Hou LG, Luo JR, Zhang JS, Zhuang LZ. Characterization of hot workability in AA7050 aluminum alloy activation energy and 3-D processing map. J Mater Proc Tech $2015 ; 225: 110-21$.

[169] Shaha SK, Czerwinski F, Kasprzak W, Friedman J, Chen DL. Effect of Zr, V and Ti on hot compression behavior of the Al-Si cast alloy for powertrain applications. J Alloy Compd 2014;615:1019-31.

[170] Bhimavarapu SB, Maheshwari AK, Bhargava D, Narayan SP. Compressive deformation behavior of Al2024 alloy using 2D and 4D processing maps. J Mater Sci 2011;46:3191:9.

[171] Liu J, Cui Z, Li C. Analysis of metal workability by integration of FEM and 3-D processing maps. J Mater Proc Tech 2008;205:497-505.

[172] Li JQ, Liu J, Cui ZS. Characterization of hot deformation behavior of extruded ZK60 magnesium alloy using 3D processing maps. Mater Des 2014;56:889-97. 
[173] Chen L, Zhao G, Gong J, Chen X, Chen M. Hot deformation behaviors and processing maps of 2024 aluminum alloy in as-cast and homogenized state. J Mater Eng Perform 2015;24:5002-12.

[174] Gangolu S, Gourav Rao A, Sabirov I, Kashyap BP, Prabhu N, Deshmukh VP. Development of constitutive relationship and processing map for Al-6-65Si-0.44Mg alloy and its composite with $\mathrm{B}_{4} \mathrm{C}$ particulates.Mater Sci Eng A 2016;655:256-64.

[175] Venugopal S, Venugopal P, Mannan SL. Optimization of cold and warm workability of commercially pure titanium using dynamic materials model (DMM) INSTABILITY MAPS. J Mater Process Technol 2008;202:201:15.

[176] Ahamed H, Senthilkumar V. Hot deformation behavior of mechanically alloyed $\mathrm{Al} 6063 / 0.75 \mathrm{Al}_{2} \mathrm{O}_{3} / 0.75 \mathrm{Y}_{2} \mathrm{O}_{3}$ nano-composite-a study using constitutive modeling and processing map. Mater Sci Eng A 2012;539:349-59.

[177] Guo HB, Wang B, Jia PR, Yang CP. In-plane shear behaviors of a 2D-SiC/SiC composite under various loading. Ceramics Inter 2015;41:11562-9.

[178] Shao JC, Xiao BL, Wang QZ, Ma ZY, Liu Y, Yang K. Constitutive flow behavior and hot workability of powder metallurgy processed $20 \mathrm{vl} . \% \mathrm{SiCp} / 2024 \mathrm{Al}$ composite. Mater Sci Eng A 2010;527:7865:72.

[179] Sakai T, Belyakov A, Kaibyshev R, Miura H, Jonas JJ. Dynamic and post-dynamic recrystallization under hot, cold, and severe plastic deformation conditions. Prog Mater Sci 2014;60:130-207.

[180] Ko BC, Yoo YC. Prediction of dynamic recrystallization condition by deformation efficiency for Al2024 composite reinforced with SiC particle. J Mater Sci 2000;35:40737. 
[181] Sani SA, Ebrahimi GR, Kiani Rashid AR. Hot deformation behavior and dynamic recrystallization kinetics of AZ61 and AZ61+Sr magnesium alloys. J Mag Alloy 2016;4:104-14. 